\title{
The Phenomenology of Neutral Heavy Leptons
}

\author{
by \\ Ivan Melo, RNDr. \\ A thesis submitted to \\ the Faculty of Graduate Studies and Research \\ in partial fulfilment of \\ the requirements for the degree of \\ Doctor of Philosophy \\ Department of Physics \\ Ottawa-Carleton Institute for Physics \\ Ottawa, Ontario \\ February, 1996 \\ (C) copyright 1996, Ivan Melo
}


The undersigned hereby recommend to the Faculty of Graduate Studies and Research acceptance of the thesis,

\section{The Phenomenology of Neutral Heavy Leptons}

submitted by Ivan Melo, RNDr. in partial fulfilment of the requirements for the degree of Doctor of Philosophy

Chair, Department of Physics

Thesis Supervisor

External Examiner

Carleton University

Date: 


\section{Abstract}

Naturally small neutrino masses can arise in some grand unified models. The mechanism of neutrino mass generation in these models typically requires the existence of neutral heavy leptons. We study the low-energy phenomenology of these new fermions. Concentrating on loop corrections due to neutral heavy leptons, we examine how the flavour-conserving leptonic decays of the $\mathrm{Z}$ boson, universality breaking in these decays, and the $\mathrm{W}$ boson mass depend on the mass and mixings of the neutral heavy leptons. Working within the framework of a superstring-inspired $S U(2)_{L} \times U(1)_{Y}$ model, we show that these flavour-conserving processes have some virtues over the traditionally considered flavour-violating decays. 


\section{Acknowledgements}

Having come to Carleton from overseas, I was lucky to find here excellent conditions for my work, from the top research carried out at the department, to even more importantly, a very friendly, stimulating and inspiring atmosphere created by the Physics faculty, staff and students. I am indebted to everyone who helped me to feel at home. Especially one person is dear to my family. In late Roselyn Tighe we found an exceptional friend who stood by us in both good and bad times. She became our Canadian mom. Thank you, Roz, for everything.

I was priviledged to work closely with two fine physicists who helped me to achieve my best. My thanks go to Pat Kalyniak who helped my dream of doing theoretical physics to come true, for her guidance and support; and to Peter Watson for the many discussions his true physics spirit made so exciting.

To my parents, for their support and prayers, I am grateful.

To Jakub, Matej and Katka, for giving me the strength to pursue my goal, my love. 


\section{Contents}

List of Tables vii

List of Figures viii

$\begin{array}{lll}1 & \text { Introduction } & 1\end{array}$

1.1 The problem of small neutrino masses . . . . . . . . . . . . . . . . . . 1

1.2 Solutions to the problem . . . . . . . . . . . . . . . . . 4

1.3 The phenomenology of NHL's . . . . . . . . . . . . . . . . . 8

2 The models of neutrino mass 12

2.1 Classical electroweak Lagrangian . . . . . . . . . . . . . . . . 13

2.2 Fermion masses in the $\mathrm{SM}$. . . . . . . . . . . . . . . . . . 18

$2.3 \quad$ Neutrino masses in $S U(2)_{L} \times U(1)_{Y}$ models beyond the SM . . . . . 21

2.3 .1 A simple model of neutrino mass . . . . . . . . . . . . . . . 21

2.3.2 See-saw mechanism in an $S U(2)_{L} \times U(1)_{Y}$ model, Majorana neutrinos and Majorana NHL's . . . . . . . . . . . . . . . 22

2.4 Neutrino mass in grand unified model. . . . . . . . . . . . . . . . . 26

3 A superstring-inspired $S U(2)_{L} \times U(1)_{Y}$ model of neutrino mass $\quad 28$

3.1 Fermion content and mass matrix . . . . . . . . . . . . . . . . . . . 29

3.1 .1 Diagonalization of $\mathcal{M}$ for a single family . . . . . . . . . . . . 30 
3.1.2 Mass matrix diagonalization in case of three families . . . . . 32

3.1 .3 Discussion of mass eigenstates . . . . . . . . . . . . . . . . . . 34

3.2 Properties of the mixing matrix . . . . . . . . . . . . . . . . 35

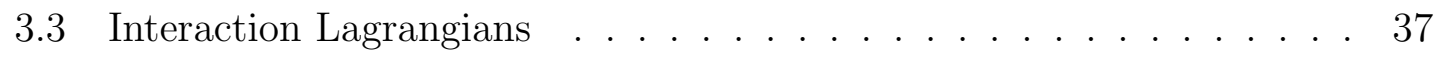

3.4 Review of existing constraints on NHL's . . . . . . . . . . . . . . . . 39

4 Standard model at the one-loop level 44

4.1 Quantization . . . . . . . . . . . . . . . . . . . . 45

4.2 Renormalization . . . . . . . . . . . . . . . . . . . 46

$4.2 .1 \quad$ Electric charge renormalization . . . . . . . . . . . . . . 47

4.2 .2 Renormalization schemes . . . . . . . . . . . . . . . . . . 50

4.2 .3 Mass renormalization . . . . . . . . . . . . . . 53

4.2 .4 Field renormalization . . . . . . . . . . . . . . . . 54

4.3 The on-shell scheme of W. Hollik . . . . . . . . . . . . . . . . 57

5 Lepton flavour-violating processes $\quad 60$

$5.1 \quad$ Flavour-violating leptonic decays of the Z boson . . . . . . . . . . . . 61

5.1 .1 The amplitude and the width for $Z \rightarrow l_{1}^{-} l_{2}^{+}$. . . . . . . . . . 62

5.1 .2 Approximate relations in the limit of large NHL mass . . . . . 66

5.1 .3 Numerical results . . . . . . . . . . . . . . . . . . 68

5.2 Flavour-violating processes at very low energies . . . . . . . . . . 70

6 Lepton flavour-conserving processes $\quad 75$

$6.1 \mathrm{Z} \rightarrow \mathrm{1}^{+} \mathrm{I}^{-}:$the tree-level and the corrections . . . . . . . . . . 76

6.2 QED corrections . . . . . . . . . . . . . . . . 77

6.3 Z-propagator corrections $\Pi_{Z} \ldots \ldots \ldots \ldots \ldots$

6.4 Vertex factor $\delta \Gamma_{l l} \ldots \ldots \ldots \ldots$

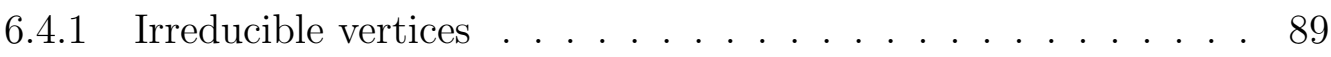

6.4 .2 Lepton self-energies . . . . . . . . . . . . . . . . . . 93 
6.4 .3 Form factors $\hat{F}_{V}, \hat{F}_{A}$ in the limit of large NHL mass . . . . . . 95

6.5 Imprecise $M_{W}$, precise $G_{\mu} \ldots \ldots \ldots$

6.6 Violation of the decoupling theorem . . . . . . . . . . . . . . . . . 101

6.7 Heavy NHL's and perturbation theory breakdown . . . . . . . . . . . 103

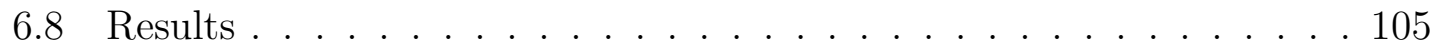

6.9 Discussion and Conclusions . . . . . . . . . . . . . . . . 110

$\begin{array}{lll}7 \text { Muon decay and W mass } & 118\end{array}$

7.1 Box diagrams $\ldots \ldots \ldots$. . . . . . . . . . . . . . . . . . 118

7.2 Neutrino self-energy and its renormalization . . . . . . . . . . . . . . 122

7.2 .1 Renormalization of the neutrino self-energies . . . . . . . . . . 124

7.2 .2 Limit $M_{N} \gg M_{W}, M_{Z}, M_{H}$. . . . . . . . . . . . . . . . 127

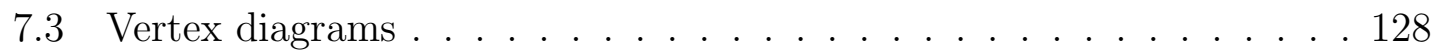

7.4 Result . . . . . . . . . . . . . . . . . . 131

8 Conclusions 133

\begin{tabular}{ll}
\hline A Dirac algebra and trace theorems & 137
\end{tabular}

A.1 Gamma matrices . . . . . . . . . . . . . . . 137

A.2 Spin sums . . . . . . . . . . . . . . . . . . . . . . . . . . . . 139

A.3 Fierz identities used for the calculation of the boxes . . . . . . . . . . 139

A.4 Proof of the identity $\nu_{L}^{c} \nu_{R}^{c}=\overline{\nu_{L}} \nu_{R} \ldots \ldots \ldots$. . . . . . . . 140

B Couplings of $\nu^{\prime}$ and $N$ to Higgs $\quad 141$

$\begin{array}{ll}\text { C Feynman rules } & 145\end{array}$

D Dimensional regularization and some useful integrals 151

D.1 Dimensional regularization . . . . . . . . . . . . . . . . . . 151

D.2 The computation of $\mathcal{I}_{0}, \mathcal{I}_{1}(m), \mathcal{I}_{2}(m)$ and $\mathcal{I}_{3}(m)$ integrals . . . . . . . 154 
E Renormalization constants, unrenormalized self-energies and 't Hooft $\begin{array}{lr}\text { scalar integrals } & 157\end{array}$

E.1 Renormalization constants . . . . . . . . . . . . . . . . . . . 157

E.2 Unrenormalized self-energies in the SM . . . . . . . . . . . . . . . . . 159

E.3 t Hooft scalar integrals . . . . . . . . . . . . . . . . . . . 161 


\section{List of Tables}

1.1 The three families of quarks $(u, c, t, d, s, b)$ and leptons (neutrinos $\nu_{e}, \nu_{\mu}, \nu_{\tau} ;$ charged leptons $\left.e, \mu, \tau\right)$ and their masses according to the standard model of electroweak interactions . . . . . . . . . . . . . . . 2

6.1 Comparison of $\hat{\Pi}_{\text {Zappx }}$ with $\hat{\Pi}_{Z} \ldots \ldots \ldots \ldots \ldots \ldots$

6.2 Comparison of $\hat{F}_{V a p p x}$ with $F_{V} \ldots \ldots \ldots \ldots$

6.3 SM limit of our model $\ldots \ldots \ldots$

$6.4 \Gamma_{0}$ and $\Gamma_{\tau \tau}$ as a function of $M_{N} \ldots \ldots \ldots \ldots$

7.1 Contribution of the muon decay loops to $\delta_{V}$ and $\Delta_{T} \ldots \ldots \ldots 132$ 


\section{List of Figures}

3.1 A one-loop diagram leading to $\mu \rightarrow e \gamma$ decay . . . . . . . . . . . . . . 42

5.1 One-loop diagrams for flavour-violating leptonic decays of the $\mathrm{Z}$ boson. 62

5.2 The branching ratio $Z \rightarrow l_{1}^{ \pm} l_{2}^{+}$as a function of $M_{N}$ for $(\mathrm{a}) \delta=-1$, (b) $\delta=+1 \ldots \ldots \ldots \ldots$. . . . . . . . . . . . . . . 71

6.1 QED corrections . . . . . . . . . . . . . . . . . . . . 78

6.2 Photon self-energy. . . . . . . . . . . . . . . . . . . . 80

6.3 Photon-Z mixing energy. . . . . . . . . . . . . . . . . . . . . . 80

6.4 Z boson self-energy . . . . . . . . . . . . . . . . . . . . . . . . . . . . 81

6.5 W boson self-energy $\ldots \ldots \ldots$. . . . . . . . . . . . . . . . . . . . . 81

6.6 Non-SM loops with NHL's and massless neutrinos. . . . . . . . . . . . 82

6.7 Irreducible vertex corrections. . . . . . . . . . . . . . . . . . . . . . . 90

6.8 Lepton self-energies. . . . . . . . . . . . . . . . . . . . . 90

6.9 Momenta and charge flow convention. . . . . . . . . . . . . . . . . . 91

6.10 The definition of the lepton self-energy $\Sigma$. . . . . . . . . . . . . 93

6.11 Diagrams with the top quark nondecoupling. . . . . . . . . . . . . . . 102

6.12 Decay modes of an NHL. . . . . . . . . . . . . . . . . . . 104 
$6.13 \mathrm{Z}$ leptonic width as a function of $M_{N}$ in the interval $0.5 \mathrm{TeV} \leq M_{N} \unlhd$ $5 \mathrm{TeV}$ for (a) fixed mixing parameter $\tau \tau_{m i x}$, fixed Higgs mass and different values of $m_{t}$; both $Z \rightarrow \tau \tau$ and $Z \rightarrow e e$ modes shown, (b) fixed mixing parameter $\tau \tau_{m i x}$, fixed $m_{t}$ and different values of the Higgs mass $M_{H}$; only $Z \rightarrow \tau \tau$ mode shown. The dashed lines represent 10 band about the current experimental value $\Gamma_{\tau \tau}^{e x p}=83.85 \pm 0.29 \mathrm{MeV}$.

$6.14 \mathrm{Z}$ leptonic width as a function of $M_{N}$ in the interval $0.5 \mathrm{TeV} \leq M_{N} \exists$ $5 \mathrm{TeV}$ for fixed $m_{t}$, fixed Higgs mass and different values of the mixing parameter, (a) $Z \rightarrow \tau \tau$ mode, (b) $Z \rightarrow e e$ mode. The dashed lines represent $1 \sigma$ band about the current experimental value (a) $\Gamma_{\tau \tau}^{\exp } \exists$ $83.85 \pm 0.29 \mathrm{MeV},(\mathrm{b}) \Gamma_{e e}^{e x p}=83.92 \pm 0.17 \mathrm{MeV}$. . . . . . . . . 112

$6.15 Z \rightarrow \tau \tau$ width as a function of $M_{N}$ in the interval $50 \mathrm{GeV} \leq M_{N} \unlhd$ $500 \mathrm{GeV}$ for a fixed top mass $m_{t}=176 \mathrm{GeV}$ and different values of the mixing parameter $\tau \tau_{m i x}$. The dashed lines represent the $1 \sigma$ band about the current experimental value $\Gamma_{\tau \tau}^{e x p}=83.85 \pm 0.29 \mathrm{MeV}$. . . 113

6.16 Universality breaking parameter $U_{b r}$ as a function of $M_{N}$ for fixed top quark mass $\left(m_{t}=176 \mathrm{GeV}\right)$ and different values of the mixing parameter. The dashed line represents $1 \sigma$ experimental limit $(<0.00245) .114$

$6.17 \mathrm{~W}$ mass as a function of $M_{N}$ for (a) fixed mixing parameter $\left(\tau \tau_{\operatorname{mix}} \exists\right.$ 0.033 ) and different values of $m_{t}$, (b) fixed top quark mass $\left(m_{t} \exists\right.$ $176 \mathrm{GeV}$ ) and different values of the mixing parameter. The dashed lines represent $1 \sigma$ band about the current experimental value $M_{W} \exists$ $80.410 \pm 0.180 \mathrm{GeV} \ldots \ldots \ldots \ldots . \ldots \ldots \ldots$

$7.1 \quad$ Box diagrams for muon decay $\ldots \ldots \ldots \ldots$

7.2 Neutrino self-energy diagrams for muon decay . . . . . . . . . . . . 123

7.3 Counterterm diagram for neutrino self-energy in muon decay . . . . . 125

7.4 Vertex diagrams for muon decay $\ldots \ldots \ldots \ldots \ldots$ 


\section{Chapter 1}

\section{Introduction}

\subsection{The problem of small neutrino masses}

Why are macroscopic things around us massive ? We know they, as composite ob-

jects, get their mass from the mass of their constituents and from the interactions among the constituents. But if the constituents are elementary particles, where is their mass coming from ? For theoretical physicists mass represents a rather unwelcome pollution of their elegant massless theories. They believe the world is essentially symmetric and it is the mass that makes this symmetry hard to see (symmetry is hidden or spontaneously broken). Therefore they build massless theories possessing beautiful symmetries and then break these symmetries to give particles their masses. The exact mechanism of symmetry breaking is not known and may not be known for a long time. It is one of the most attractive problems of particle physics. Some argue we have to wait for the ultimate solution until physics at the Planck scale $\left(10^{19} \mathrm{GeV}\right)$ is understood, others suggest optimistically that $10^{5} \mathrm{GeV}$ might do. The ultimate theory should predict the masses of all elementary particles in agreement with experiment.

It is believed the origin of the weak boson masses (associated with the electroweak 
symmetry breaking) is better understood than that of the fermion masses (flavour symmetry breaking). Concentrating on the fermions, we list the quark and lepton content of the so-called standard model of electroweak interactions in Table 1.1, along with their masses [1]. These masses are not predicted by the standard model.

\begin{tabular}{|l|c|}
\hline 1. & Mass $[\mathrm{MeV}]$ \\
\hline$u$ & 4.2 \\
$d$ & 7.5 \\
$\nu_{e}$ & 0 \\
$e$ & 0.5110 \\
\hline
\end{tabular}

\begin{tabular}{|l|c|}
\hline 2. & Mass $[\mathrm{MeV}]$ \\
\hline$c$ & 1100 \\
$s$ & 150 \\
$\nu_{\mu}$ & 0 \\
$\mu$ & 105.6 \\
\hline
\end{tabular}

\begin{tabular}{|l|c|}
\hline 3. & Mass $[\mathrm{GeV}]$ \\
\hline$t$ & 176 \\
$b$ & 4.2 \\
$\nu_{\tau}$ & 0 \\
$\tau$ & 1.784 \\
\hline
\end{tabular}

Table 1.1: The three families of quarks $(u, c, t, d, s, b)$ and leptons (neutrinos $\nu_{e}, \nu_{\mu}, \nu_{\tau}$; charged leptons $\left.e, \mu, \tau\right)$ and their masses according to the standard model of electroweak interactions

The quark and charged lepton masses represent an experimental input, 9 parameters out of the total 17 present in the standard model ॠ. The neutrinos are postulated as massless. This postulate, however, is based on the assumption that the neutrino is the only fermion without a right-handed field - an asymmetry going against the spirit of symmetric theories which work with both left-handed and right-handed fields. Therefore the massless neutrinos are not natural in the standard model and none of the twelve fermion masses is actually predicted.

While the ultimate solution for mass prediction may be far from us, it is worthwhile to think of partial steps which could bring us closer to it. A popular strategy aims at the reduction of the twelve independent mass parameters. From grand unified theories (GUT's) which describe strong and electroweak interactions as different manifestations of a single force, there are hints the fermion masses are related to each other by simple formulae at very high energies (GUT scale $\sim 10^{16} \mathrm{GeV}$ ) $\mathrm{f}$ and it is in

\footnotetext{
${ }^{1}$ Of the remaining 8 parameters, the four CKM mixing matrix parameters are also closely related to the origin of the quark masses.

${ }^{2}$ For example in the simplest version of $S O(10)$ GUT, all masses in a given family are equal at
} 
the transition to our low-energy world [ ] where masses pick up different corrections and end up in the array of seemingly unrelated numbers shown in Table 1.1 .

In the past the efforts to partially explain fermion masses in grand unified models experienced great difficulties facing the problem of small neutrino masses. In the discussion above, we dismissed the way the standard model postulates the zero mass neutrinos. What is then the experimental basis for the claim the neutrino masses are small, possibly zero ? Ironically, even though neutrinos are possibly the second most abundant elementary species in the Universe, we do not know exactly what their mass is. This is partly due to the smallness of this mass and partly because it is so hard to detect neutrinos (a typical cross section is $10^{-43} \mathrm{~cm}^{2}$ for a $10 \mathrm{MeV}$ neutrino, more than twenty orders of magnitude below the cross section for an electron of the same energy). Experiments set the following upper limits on the masses of the electron, muon and tau type neutrino [2]:

$$
\begin{aligned}
m_{\nu_{e}} & <7.2 \mathrm{eV} \\
m_{\nu_{\mu}} & <270 \mathrm{keV} \\
m_{\nu_{\tau}} & <31 \mathrm{MeV}
\end{aligned}
$$

The neutrino masses are, even at their allowed maxima, strikingly small when compared with the masses of the charged leptons and quarks within their families. For example, within the first family of the standard model, $u$ and $d$ quarks have masses of a few MeV, and the electron has a mass of $0.511 \mathrm{MeV}$ (see Table 1.1). The simple formulae relating the fermion masses we mentioned earlier, put different fermion masses on the same scale: it is thus conceivable in GUT's that masses of the first family are scattered around a few $\mathrm{MeV}$, but the mass of the electron type neutrino is a deep mystery, being at least five orders of magnitude below this natural scale

the GUT scale.

${ }^{3}$ By low energies we mean here energies up to a few hundred $\mathrm{GeV}$ and by very low energies those up to a few $\mathrm{GeV}$. 
for the first family. The difference of five orders cannot be explained by corrections masses pick up running from the GUT scale to low energies. Something else must be involved. Similar behaviour, although perhaps less pronounced, can be observed among the second and third family members.

\subsection{Solutions to the problem}

Before we start to discuss possible solutions to our problem, let us be more specific about the relation between GUT's and low-energy theories, such as the standard model. While the standard model [3, 田, 5, 6] has a natural scale of $10^{2} \mathrm{GeV}$, GUT models [7] describe physics actively operating at the GUT scale of $10^{16} \mathrm{GeV}$; at the same time they should explain low-energy data at least as well as the standard model. GUT models, although more elegant than the standard model, are also more complicated: they have a richer gauge structure and symmetry breaking sector and, especially in the case of $E_{6}$, a fermion sector with more particles. A thorough discussion of neutrino masses in these models is beyond the scope of this work. Here we are mainly interested in the phenomenology of neutral heavy leptons at low energies. Fortunately, at low energies most of the extraneous baggage associated with the complicated structure of GUT models has very little impact - it is integrated out and the remaining effective theory often represents just a minimal extension of the standard model of electroweak interactions. This is in fact no big surprise. It simply reflects a very good agreement of experimental data with the standard model and the fact the GUT's extend the standard model rather than replace it. The physics active at high energies thus decouples at the scales currently accessible to us. For example, in the model studied in this thesis, it is just two extra neutrino fields per family which enlarge the standard model. Therefore we will focus our discussion on these minimal extensions, referring to unification models as motivation for a particular simple extension of the standard model. Interestingly, basic directions 
in the theoretical treatment of neutrino masses can be followed with just minor extensions of the fermion sector of the standard model, leaving the gauge structure $S U(2)_{L} \times U(1)_{Y}$ and symmetry breaking sector intact.

The simplest model with massive neutrinos one can immediately think of is a straightforward extension of the standard model. One can introduce the righthanded neutrino field missing in the standard model and treat the neutrino in the same way as all other fermions - as a massive Dirac particle. This means the neutrino mass is still not predicted and the simplest model thus fails to address the problem of the small neutrino masses.

A possible solution was found by Yanagida and Gell-Mann, Ramond and Slansky in the famous see-saw mechanism [8, 9]. A simple low-energy see-saw model has the same fermion content as the simplest model just described, however, mass terms violating the total lepton number $L$ are allowed. This leads to the description of neutrinos as Majorana fermions rather than Dirac; neutral heavy leptons (NHL's) are introduced into this theory as a necessary ingredient. In Chapter 2 we describe the difference between Majorana and Dirac fermions more formally. Here it suffices to say that a Dirac neutrino is a particle like all other fermions with left-handed and right-handed particle and antiparticle states, while a Majorana neutrino is a particle which is its own antiparticle and therefore comes in just two states described by a left-handed and a right-handed field. The neutrino is the only particle of the fermion content of the standard model that can possibly be described as a Majorana particle because it is neutral.

The see-saw mechanism comes with the following relation for the mass of a neutrino $m_{\nu}$ :

$$
m_{\nu}=D^{2} / M_{N} .
$$

The mass $D$ is a typical family mass (say $1-2 \mathrm{MeV}$ for the first family) and $M_{N}$ is 
the NHL mass. This relation tells us the neutrinos become very light with respect to $D$ due to a very large mass $M_{N}$. Their mass is not predicted ( $M_{N}$ is unknown); nevertheless its smallness is understood since a very large mass scale required for the $M_{N}$ is naturally expected in GUT's.

Whether neutrinos are Dirac or Majorana particles is an issue by itself. Experiments which try to address it rely on the fact that Majorana neutrinos break lepton number conservation, whereas Dirac neutrinos respect it. A clear answer would be provided by the observation of a neutrinoless double beta decay, a so far unobserved process mediated only by Majorana neutrinos 1 [11].

Theories with massive neutrinos are popular since some motivation for nonzero neutrino masses comes also from outside particle physics. Massive neutrinos could explain the mystery of missing solar neutrinos [12] through matter enhanced time dependent neutrino oscillations, the so-called MSW effect [13]. In cosmology, massive neutrinos could explain at least part of the dark matter puzzle. The possibility of this increased after COBE data on the anisotropy of the cosmic microwave background radiation were analysed: there are hints some $10-30 \%$ of the dark matter is hot [14] and neutrinos with masses between $2-7 \mathrm{eV}$ make a good candidate. Cosmology further constrains (subject to plausible assumptions) the mass of each of the three neutrinos to $m_{\nu}<25 \mathrm{eV}$ (see Ref. [15], Sec. 15.3.1).

These astrophysical and cosmological indications are quite appealing and some authors argue on their basis that an $S O(10)$ GUT with three nearly degenerate Majorana neutrino masses of about $2 \mathrm{eV}$ could be the correct unified theory [16].

The see-saw mechanism is not the only one which addresses the issue of the

\footnotetext{
${ }^{4}$ Another possibility of proving the Majorana nature of the neutrino was suggested as a result of theoretical studies of the origin of the lepton symmetry breaking. This symmetry can be broken explicitly (it is not a symmetry of the Lagrangian at any energy or temperature); it can also be broken spontaneously. If the lepton number $L$ is a global symmetry which is broken spontaneously, a massless, pseudoscalar Majoron arises [10]. The discovery of this particle would prove the Majorana nature of the neutrino.
} 
smallness of the neutrino mass. An alternative was worked out in the class of models wherein neutrinos remain massless while other neutral leptons can acquire a large mass. Here it is argued there can be some global symmetry present, such as lepton number $L$, which prevents neutrinos from becoming massive. One such model could arise as a low-energy limit of a superstring-inspired $E_{6}$ GUT [17]. The superstring inspiration consists of yet another (left-handed) neutrino field added to the fermion content of the standard model. Therefore we have three neutrino fields per family: the standard left-handed one, the right-handed one required by GUT's and the one needed by superstrings. This field content and the lepton number symmetry 回 give rise to three massless neutrinos and to three massive Dirac NHL's, as described in Chapter 3. In this thesis I study phenomenological implications of this model (henceforth called 'our' model), with emphasis put on signatures of NHL's in precision data from LEP collider at CERN (leptonic widths of the Z boson) and Tevatron collider at Fermilab (the mass of the $\mathrm{W}$ boson, $M_{W}$ ).

Both theories with massive and massless neutrinos (our model) naturally exhibit some of the properties familiar from the quark sector of the standard model. For instance, neutrino mixing may arise with an analogue of the Cabibbo-KobayashiMaskawa (CKM) mixing matrix for the leptonic sector. Individual lepton family numbers are expected to be violated, as is lepton universality and CP symmetry. The total lepton number $L$ has already been discussed. What is not predicted by our model, in contrast with see-saw models, are time dependent neutrino oscillations and neutrinoless double beta decay. The physics of light neutrinos is thus richer in see-saw models. On the other hand the physics of neutral heavy leptons looks more promising in our model. The NHL's contribution to low-energy observables is

\footnotetext{
${ }^{5}$ We note the conserved lepton number is also present in the standard model and in the simplest model with massive neutrinos. However, in the former the conserved $L$ is a consequence of the missing right-handed neutrino field and in the latter, the two neutrino fields per family are not enough to keep neutrinos massless.
} 
proportional to their mixings and these depend on the ratio $\frac{D}{M_{N}}$. In see-saw models

this ratio is normally very small since the ratio $\frac{D^{2}}{M_{N}}=m_{\nu}$ is very small. In our model with massless neutrinos there is no such restriction and, consequently, mixings can be relatively large.

To show that the differences between theories with massive neutrinos and our model should not be taken too seriously, we note that there is a variant of our model where the lepton number symmetry is slightly broken and the neutrinos are given a small mass [17, 18] and there are variants of see-saw models where the restriction from the ratio $\frac{D^{2}}{M_{N}}$ is avoided (assuming certain symmetries in the neutrino mass matrix) and possibly large mixings of NHL's arise as a result [19, 20].

\subsection{The phenomenology of NHL's}

To investigate the phenomenology of NHL's more closely, let us be more specific about how they enter low-energy observables via their mixings. For simplicity we neglect interfamily mixings in this section. The key point, formally derived and discussed in Chapters 2 and 3, is that the neutrinos taking part in weak interactions, $\nu_{l}(l=e, \mu, \tau)$ are no longer states of definite mass but are combinations of light (massless in our model) neutrinos $\nu^{\prime}$ and NHL's $N$ :

$$
\nu_{l}=K_{L} \nu^{\prime}+K_{H} N
$$

Here $K_{L}$ and $K_{H}$ are mixing parameters related through $K_{L}^{2}+K_{H}^{2}=1$. The chance of finding the signature of an NHL is proportional to the size of $K_{H}$, which is equal to the ratio we discussed in the previous section, $K_{H}=\frac{D}{M_{N}}$. This picture is model independent, whether NHL's come from our model, or a see-saw one. In the standard model we have $K_{H}=0, K_{L}=1$ and the weak eigenstates $\nu_{l}$ become identical with the mass eigenstates $\nu^{\prime}$. 
The eigenstates $\nu_{l}$ interact weakly by coupling to the mediators of electroweak interactions, $\mathrm{W}$ and $\mathrm{Z}$ bosons. Through the mixing $K_{H}$, so do NHL's. For example, the simplest way to discover NHL's would be to directly produce them at the $\mathrm{Z}$ boson factories (LEP collider at CERN and SLC at SLAC). However, to be produced directly, their mass would have to be smaller than the $\mathrm{Z}$ boson mass - this is the energy available at the $\mathrm{Z}$ factories. Since there has been so far no evidence for NHL production, we conclude that either they are more massive than the Z, or their mixing is small enough to suppress their production to such a degree that they escape detection.

Another method to probe mixings of NHL's is an indirect one, through the measurement of the mixing parameter $K_{L}$ of the light neutrinos in, for example, pion and beta decays. NHL's are not produced in the decays, nevertheless their existence could be revealed if $K_{L}$ is far enough from 1 to reduce the decay rates beyond the experimental uncertainties. These so-called universality constraints give us the best limits on mixings.

While the direct production is sensitive to NHL masses only up to the mass of the $\mathrm{Z}$ boson $M_{Z}$, indirect methods are sensitive only to mixings, not masses. What if the NHL is heavier than the $\mathrm{Z}$ boson ? Can we obtain some information on its mass ? In cases when particle physicists face a problem of probing masses of hypothetical particles which are larger than the energy currently available, they study contributions of these particles in radiative corrections (loops) to some observables. Since loops are higher order terms of perturbation theory, they represent just a small correction to the lowest order (tree-level) calculation and often their size is not greater than experimental uncertainties. For example, only recently have precision experiments seen some evidence for the genuine electroweak loop corrections of the standard model. Despite their smallness, loop corrections in combination with precision data can impose important restrictions on the parameter space of various models. 
The major part of my thesis studies how NHL's contribute via loops to the regular leptonic decays of the $\mathrm{Z}$ boson, a lepton universality breaking parameter (both studied in precision tests at LEP) and the mass of the $\mathrm{W}$ boson. This is a novel approach to the study of NHL's in loops. The previous studies concentrated mainly on flavour-violating decays, such as $\mu \rightarrow e \gamma$ at very low energies or $Z \rightarrow e^{+} \mu^{-}$ at the Z factories' energy. We argue that our calculations probe NHL masses and mixings more efficiently than these traditional studies of flavour-violating processes. The limits on NHL mass we obtain from the leptonic decays of the $\mathrm{Z}$ boson and the lepton universality breaking parameter are comparable to the limit derived from the considerations of perturbative theory breakdown, discussed in Sec. 6.7.

This work is organized as follows: In Chapter 2 we treat models of neutrino mass formally. After specifying the classical Lagrangian of the standard model, we investigate how the peculiar nonzero energy density of the vacuum associated with the existence of a fundamental scalar Higgs field accommodates fermion masses in the standard model. We then move on to describe neutrino masses in $S U(2)_{L} \times$ $U(1)_{Y}$ models beyond the standard model; we consider the simplest extension of the standard model leading to massive Dirac neutrinos and a see-saw model with Majorana neutrinos as examples. We also briefly describe neutrino mass in grand unified models and show where motivation for our model comes from.

In Chapter 3 we discuss our model, a superstring-inspired $S U(2)_{L} \times U(1)_{Y}$ model of neutrino mass, in detail. We define the fermion content and the neutrino mass matrix and show how massless neutrinos and NHL's arise through the diagonalization of the mass matrix in the case of one family and also in the general case of three families. The mixing matrix is described and phenomenologically relevant mixing parameters are defined. In the second part of that chapter we review existing constraints on NHL's.

As a prerequisite for one-loop calculations, the standard model at the one-loop level is discussed formally in Chapter 4. A key ingredient of the calculations is the 
renormalization of the standard model and we spend some time dealing with its salient features.

In Chapter 5 we revisit the flavour-violating leptonic decays of the $\mathrm{Z}$ boson in our model. Our results are followed by a discussion of flavour-violating processes in general.

In Chapter 6 we study the impact NHL's have through loops on flavour-conserving leptonic decays of the $\mathrm{Z}$ boson, lepton universality breaking in these decays, and the W boson mass. One-loop corrections are classified and calculated and the most important diagrams are identified. Violation of the decoupling theorem by the NHL's and its relevance is discussed. Implicit dependence of our results on muon decay is clarified.

Chapter 7 completes calculations from the previous chapter by considering the full set of diagrams contributing to the muon decay.

In the last chapter we conclude by summarizing our main results. 


\section{Chapter 2}

\section{The models of neutrino mass}

In this chapter we treat the models of neutrino mass formally. We start with the standard model, then we proceed with models beyond the SM. As discussed in the Introduction, we concentrate mainly on minimal extensions of the standard model models with $S U(2)_{L} \times U(1)_{Y}$ gauge structure.

In Sec. 2.1 we present the classical electroweak Lagrangian defining the standard model. We rather state than discuss the principles upon which it is based. The purpose is to specify our notation and to give a practical reference point for the discussions of the models of neutrino mass (Chapters 2 and 3) and for one-loop calculations (Chapters 5,6,7) 丹.

In Sec. 2.2 we examine the fermion masses in the standard model, in Sec. 2.3 we describe the generation of neutrino masses in simple extensions of the standard model (a simple model with Dirac neutrinos and a see-saw model with Majorana neutrinos) and finally, in Sec. 2.4 we briefly deal with neutrino masses in grand unified models. The superstring-inspired minimal extension of the standard model, motivated in this last section, is discussed in detail in Chapter 3.

\footnotetext{
${ }^{1}$ One-loop calculations actually require the quantization and the subsequent extension of the classical Lagrangian by additional terms; this is discussed in Chapter 4, which directly precedes one-loop calculations in Chapters 5,6 and 7 .
} 


\subsection{Classical electroweak Lagrangian}

The standard model of electroweak interactions (SM) [3, 4, 5, 6] is a gauge theory based on a local $S U(2)_{L} \times U(1)_{Y}$ symmetry group, which describes electromagnetic and weak interactions as manifestations of a single electroweak force. The $S U(2)_{L}$ part is the group of the weak isospin $I$ and the $U(1)_{Y}$ part is the group of the weak hypercharge $Y$. The quantum numbers $I_{3}$ (the third component of $I$ ) and $Y$ are related to the electric charge $Q$ via

$$
Q=I_{3}+\frac{Y}{2}
$$

The $S U(2)_{L} \times U(1)_{Y}$ symmetry is spontaneously broken to $U(1)_{e m}$ (the group of the electric charge $Q$ ) and particle masses are generated by the nonsymmetric vacuum.

We now list the fermion content of the SM 27. The particles, represented by chiral (left-handed and right-handed) fields, form three families and therefore can be represented as three-component vectors in family space. With the theoretical treatment of fermion masses in mind we differentiate between the field content of an unbroken electroweak theory and that of a broken one. The left-handed lepton fields of the unbroken theory,

$$
\psi_{L} \equiv\left(\psi_{1_{L}}, \psi_{2_{L}}, \psi_{3_{L}}\right)=\left[\left(\begin{array}{c}
\nu_{e} \\
e
\end{array}\right)_{L},\left(\begin{array}{c}
\nu_{\mu} \\
\mu
\end{array}\right)_{L},\left(\begin{array}{c}
\nu_{\tau} \\
\tau
\end{array}\right)_{L}\right],
$$

transform as doublets $\left(I=\frac{1}{2}\right)$ under $S U(2)_{L}$ with the hypercharge $Y=-1$. In short, their quantum numbers are $\left(\frac{1}{2},-1\right)$.

The right-handed lepton fields $(0,-2)$ are

$$
\psi_{R} \equiv\left(\psi_{1_{R}}, \psi_{2_{R}}, \psi_{3_{R}}\right)=\left(e_{R}, \mu_{R}, \tau_{R}\right)
$$

\footnotetext{
${ }^{2}$ In fact, we already did it in Table 1.1; here we use more formal description.
} 
Note there are no right-handed neutrino fields in the SM.

The left-handed $\left(\frac{1}{2}, \frac{1}{3}\right)$ and the right-handed up $\left(0, \frac{4}{3}\right)$ and down $\left(0,-\frac{2}{3}\right)$ quark fields of the unbroken theory are respectively

$$
\begin{aligned}
q_{L}^{\prime} & \equiv\left(q_{1_{L}}^{\prime}, q_{2_{L}}^{\prime}, q_{3_{L}}^{\prime}\right)=\left[\left(\begin{array}{l}
u_{1}^{\prime} \\
d_{1}^{\prime}
\end{array}\right)_{L},\left(\begin{array}{l}
u_{2}^{\prime} \\
d_{2}^{\prime}
\end{array}\right)_{L},\left(\begin{array}{l}
u_{3}^{\prime} \\
d_{3}^{\prime}
\end{array}\right)_{L}\right] \\
u_{R}^{\prime} & \equiv\left(u_{1_{R}}^{\prime}, u_{2_{R}}^{\prime}, u_{3_{R}}^{\prime}\right) \\
d_{R}^{\prime} & \equiv\left(d_{1_{R}}^{\prime}, d_{2_{R}}^{\prime}, d_{3_{R}}^{\prime}\right) .
\end{aligned}
$$

The quark fields (weak eigenstates) of the broken theory are different from the fields of the unbroken theory (and also from the quark mass eigenstates) :

$$
\begin{aligned}
q_{L} & \equiv\left(q_{1_{L}}, q_{2_{L}}, q_{3_{L}}\right)=\left[\left(\begin{array}{c}
u \\
\tilde{d}
\end{array}\right)_{L},\left(\begin{array}{c}
c \\
\tilde{s}
\end{array}\right)_{L},\left(\begin{array}{l}
t \\
\tilde{b}
\end{array}\right)_{L}\right], \\
u_{R} & \equiv\left(u_{1_{R}}, u_{2_{R}}, u_{3_{R}}\right)=\left(u_{R}, c_{R}, t_{R}\right), \\
\tilde{d_{R}} & \equiv\left(\tilde{d_{1}}, \tilde{d_{2}}, \tilde{d_{3_{R}}}\right)=\left(\tilde{d_{R}}, \tilde{s_{R}}, \tilde{b_{R}}\right),
\end{aligned}
$$

where $\tilde{d}=V_{C K M} d$ are weak eigenstates of the broken theory obtained from mass eigenstates $d$ through the Cabibbo-Kobayashi-Maskawa (CKM) matrix $V_{C K M}$ (see also Eq. 2.27).

The classical electroweak Lagrangian is the sum of the fermion part, the gauge part and the Higgs part:

$$
\mathcal{L}_{E W}=\mathcal{L}_{G}+\mathcal{L}_{F}+\mathcal{L}_{H} .
$$

The fermion part, which describes the fermions and their interactions, is given 
by

$$
\begin{aligned}
\mathcal{L}_{F} & =i \sum_{j=1}^{3}\left\{\overline{\psi_{j_{L}}} \gamma^{\mu} \mathcal{D}_{\mu} \psi_{j_{L}}+\overline{\psi_{j_{R}}} \gamma^{\mu} \mathcal{D}_{\mu} \psi_{j_{R}}+\overline{q_{j_{L}}} \gamma^{\mu} \mathcal{D}_{\mu} q_{j_{L}}\right. \\
& \left.+\overline{u_{j_{R}}} \gamma^{\mu} \mathcal{D}_{\mu} u_{j_{R}}+\overline{d_{j_{R}}} \gamma^{\mu} \mathcal{D}_{\mu} d_{j_{R}}\right\}
\end{aligned}
$$

where

$$
\begin{aligned}
\mathcal{D}_{\mu} \psi_{L} & =\left[\left(\partial_{\mu}+i \frac{g_{1}}{2} Y B_{\mu}\right) \mathbf{I}-i \frac{g_{2}}{2} \overrightarrow{\boldsymbol{\tau}} \cdot \vec{W}_{\mu}\right] \psi_{L}, \\
\mathcal{D}_{\mu} \psi_{R} & =\left(\partial_{\mu}+i \frac{g_{1}}{2} Y B_{\mu}\right) \psi_{R},
\end{aligned}
$$

are covariant derivatives for left and right-handed fields respectively. These derivatives ensure the gauge invariance of the $\mathcal{L}_{F}$ by introducing a weak isospin triplet of gauge fields $\vec{W}_{\mu} \equiv\left(W_{\mu}^{1}, W_{\mu}^{2}, W_{\mu}^{3}\right)$ and a weak isospin singlet gauge field $B_{\mu}$. The gauge fields interact with the fermions with the strength $g_{2}$, the $S U(2)_{L}$ coupling constant and the strength $g_{1}$, the $U(1)_{Y}$ coupling constant. $\mathbf{I}$ is a $2 \times 2$ unit matrix in isospin space and $\frac{1}{2} \vec{\tau}$ are generators of $S U(2)_{L}$ transformations in two-dimensional representation; $\overrightarrow{\boldsymbol{\tau}}$ are Pauli matrices (see Appendix A). Weak isospin $I$ is the eigenvalue of the operator $\left(\frac{1}{2} \vec{\tau}\right)^{2}$ and $I_{3}$ is the eigenvalue of the operator $\frac{1}{2} \tau_{3}$.

The gauge part of the Lagrangian describes the gauge fields and their self-interactions; it is given by

$$
\mathcal{L}_{G}=-\frac{1}{4} W_{\mu \nu}^{a} W^{\mu \nu, a}-\frac{1}{4} B_{\mu \nu} B^{\mu \nu},
$$

where $a=1,2,3$ is the $S U(2)$ index and

$$
\begin{aligned}
W_{\mu \nu}^{a} & =\partial_{\mu} W_{\nu}^{a}-\partial_{\nu} W_{\mu}^{a}+g_{2} \epsilon_{a b c} W_{\mu}^{b} W_{\mu}^{c} \\
B_{\mu \nu} & =\partial_{\mu} B_{\nu}-\partial_{\nu} B_{\mu}
\end{aligned}
$$


are field strength tensors for the isotriplet $W_{\mu}^{a}$ and the isosinglet $B_{\mu}$ fields respectively.

The Higgs part of the Lagrangian, responsible for spontaneous electroweak symmetry breaking, is the sum of two terms:

$$
\mathcal{L}_{H}=\mathcal{L}_{H G}+\mathcal{L}_{H F}
$$

Here $\mathcal{L}_{H G}$ describes the Higgs-gauge interactions and $\mathcal{L}_{H F}$ the Higgs-fermion or so-called Yukawa interactions. $\mathcal{L}_{H G}$ has the form

$$
\mathcal{L}_{H G}=\left(\mathcal{D}_{\mu} \Phi\right)^{\dagger}\left(\mathcal{D}^{\mu} \Phi\right)-V(\Phi)
$$

where

$$
\begin{aligned}
\Phi & =\left(\begin{array}{c}
\phi^{+} \\
\phi^{0}
\end{array}\right), \\
\mathcal{D}_{\mu} \Phi & =\left[\left(\partial_{\mu}+i \frac{g_{1}}{2} Y B_{\mu}\right) \mathbf{I}-i \frac{g_{2}}{2} \overrightarrow{\boldsymbol{\tau}} \cdot \vec{W}_{\mu}\right] \Phi, \\
V(\Phi) & =-\mu^{2} \Phi^{\dagger} \Phi+\lambda\left(\Phi^{\dagger} \Phi\right)^{2}, \quad \lambda>0 .
\end{aligned}
$$

$\mathcal{D}_{\mu} \Phi$ is the covariant derivative for the $Y=1$ Higgs doublet $\Phi\left(\frac{1}{2}, 1\right)$ with the charged component $\phi^{+}$and the neutral component $\phi^{0} ; V(\Phi)$ is the Higgs potential constructed so it can lead to the vacuum in which the average value (vacuum expectation value) of the Higgs doublet, denoted $\langle\Phi\rangle$, is nonzero. To keep $U(1)_{e m}$ unbroken, it is the neutral component $\phi^{0}$ which develops the vacuum expectation 
value:

$$
\langle\Phi\rangle=\frac{1}{\sqrt{2}}\left(\begin{array}{l}
0 \\
v
\end{array}\right), \quad v=\frac{\mu}{\sqrt{\lambda}} .
$$

The symmetry is broken spontaneously because the electroweak Lagrangian is symmetric under $S U(2)_{L} \times U(1)_{Y}$ transformations but the lowest energy state, the vacuum, is not (here $\langle\Phi\rangle$ is not symmetric).

The Higgs doublet can be written now as

$$
\Phi=\left(\begin{array}{c}
\phi^{+} \\
\phi^{0}
\end{array}\right)=\left(\begin{array}{c}
\phi^{+} \\
\frac{1}{\sqrt{2}}(v+H+i \chi)
\end{array}\right)
$$

where $\phi^{ \pm}$and $\chi$ are unphysical Higgs fields and $H$ is the physical Higgs field.

The spontaneous symmetry breaking gives rise to massive gauge fields $W_{\mu}^{ \pm}$and $Z_{\mu}$, mediators of weak charged and neutral interactions leaving the massless photon field $A_{\mu}$, the mediator of electromagnetic interactions:

$$
\begin{aligned}
W_{\mu}^{ \pm} & =\frac{1}{\sqrt{2}}\left(W_{\mu}^{1} \mp i W_{\mu}^{2}\right), \\
Z_{\mu} & =+\cos \theta_{W} W_{\mu}^{3}+\sin \theta_{W} B_{\mu}, \\
A_{\mu} & =-\sin \theta_{W} W_{\mu}^{3}+\cos \theta_{W} B_{\mu} .
\end{aligned}
$$

The $W^{ \pm}$mass $M_{W}$ and the $Z$ mass $M_{Z}$ are given by

$$
M_{W}=\frac{v}{2} g_{2}, \quad M_{Z}=\frac{v}{2} \sqrt{g_{1}^{2}+g_{2}^{2}}
$$

The Weinberg angle $\theta_{W}$ is defined as

$$
\cos \theta_{W}=\frac{M_{W}}{M_{Z}}=\frac{g_{2}}{\sqrt{g_{1}^{2}+g_{2}^{2}}} .
$$


The electric charge $e=\sqrt{4 \pi \alpha}$ can be expressed as

$$
e=\frac{g_{1} g_{2}}{\sqrt{g_{1}^{2}+g_{2}^{2}}},
$$

or

$$
g_{2}=\frac{e}{\sin \theta_{W}}, \quad g_{1}=\frac{e}{\cos \theta_{W}} .
$$

The second term of the Higgs part of the Lagrangian, $\mathcal{L}_{H F}$, is discussed in the next section.

\subsection{Fermion masses in the SM}

The spontaneous symmetry breaking is responsible also for fermion masses. The starting point is $\mathcal{L}_{H F}$ which describes the Yukawa interactions between fermions and the Higgs doublet:

$$
\begin{aligned}
& \mathcal{L}_{H F}=-\sum_{i=1}^{3} \sum_{j=1}^{3}\left[\tilde{G}_{i j} \overline{u_{i_{R}}^{\prime}}\left(\tilde{\Phi}^{\dagger} q_{j_{L}}^{\prime}\right)+G_{i j} \overline{d_{i_{R}}^{\prime}}\left(\Phi^{\dagger} q_{j_{L}}^{\prime}\right)\right]+\text { h.c. } \\
& -\quad \sum_{i=1}^{3}\left[\quad+h_{i} \overline{\psi_{i_{R}}}\left(\Phi^{\dagger} \psi_{i_{L}}\right)\right]+\text { h.c. }
\end{aligned}
$$

where

$$
\tilde{\Phi}=i \tau_{2} \Phi^{*}=\left(\begin{array}{c}
\phi^{0^{*}} \\
-\phi^{-}
\end{array}\right)
$$

is the $Y=-1$ Higgs doublet $\left(\frac{1}{2},-1\right)$ and $\tilde{G}_{i j}, G_{i j}, h_{i}$ are arbitrary Yukawa couplings which are free parameters in the SM. The purpose of the empty space in the second line of Eq. 2.22 will be clarified below. 
To generate masses, one substitutes in Eq. 2.22 the vacuum expectation value $\langle\Phi\rangle$ (see Eq. 2.15) for $\Phi$. Thus the first term (plus its h.c.) in the first line of Eq. 2.22 gives mass to $u, c, t$ quarks; the second term gives mass to $d, s, b$ quarks and the term in the second line gives mass to charged leptons.

Let us study the charged lepton case first. We get the electron mass $m_{e}$ from the second line of Eq. 2.22 for $i=1$. The substitution of $\langle\Phi\rangle$ yields

$$
\begin{aligned}
& -\frac{1}{\sqrt{2}} h_{1} \overline{e_{R}}\left(\begin{array}{l}
0 \\
v
\end{array}\right)^{\dagger}\left(\begin{array}{c}
\nu_{e_{L}} \\
e_{L}
\end{array}\right)-\frac{1}{\sqrt{2}} h_{1} \overline{\left(\begin{array}{c}
\nu_{e_{L}} \\
e_{L}
\end{array}\right)}\left(\begin{array}{l}
0 \\
v
\end{array}\right) e_{R} \\
& =-\frac{1}{\sqrt{2}} h_{1} \overline{e_{R}} v e_{L}-\frac{1}{\sqrt{2}} h_{1} \overline{e_{L}} v e_{R}=-\frac{1}{\sqrt{2}} h_{1} v\left(\overline{e_{R}} e_{L}+\overline{e_{L}} e_{R}\right) \\
& \equiv-m_{e}\left(\overline{e_{R}} e_{L}+\overline{e_{L}} e_{R}\right)=-m_{e} \bar{e} e,
\end{aligned}
$$

which is the familiar form of the Dirac mass term. Without any inter-generation couplings in the lepton part of Eq. 2.22 (Yukawa couplings $h_{i}$ are simple numbers as opposed to matrices $\tilde{G}_{i j}, G_{i j}$ ), there are no mixings among leptons in the SM 5 . As a result, lepton family numbers (flavours) are separately conserved and there are no lepton flavour-violating processes. The total lepton number $L$ is also conserved since it is the sum of lepton family numbers.

Quark masses are more involved, because inter-generation couplings are allowed $\left(\tilde{G}_{i j}, G_{i j}\right.$ are nondiagonal matrices in flavour space). As a consequence, $q_{j_{L}}^{\prime}, u_{i_{R}}^{\prime}$ and $d_{i_{R}}^{\prime}$, the weak eigenstates of the unbroken theory, are different from the mass eigen-

\footnotetext{
${ }^{3}$ When discussing fermion masses, one cannot avoid the question of possible mixings among fermions. It is because we look for mass effects in various weak processes where the states of definite weak quantum numbers (weak interaction eigenstates) participate rather than the states of definite mass (mass eigenstates). Mixings then relate mass eigenstates to weak eigenstates. Further, mixings and masses are connected through their common origin derived from Yukawa couplings and the vacuum expectation value of the Higgs doublet. For these reasons we will study mixings along with masses.
} 
states $u, c, t$ and $d, c, b$. They are related through the unitary matrices $A_{L}, A_{R}, B_{L}$, $B_{R}, 21:$

$$
\left(\begin{array}{c}
u_{1_{L, R}}^{\prime} \\
u_{2_{L, R}}^{\prime} \\
u_{3_{L, R}}^{\prime}
\end{array}\right)=A_{L, R}\left(\begin{array}{c}
u_{L, R} \\
c_{L, R} \\
t_{L, R}
\end{array}\right), \quad\left(\begin{array}{c}
d_{1_{L, R}}^{\prime} \\
d_{2_{L, R}}^{\prime} \\
d_{3_{L, R}}^{\prime}
\end{array}\right)=B_{L, R}\left(\begin{array}{c}
d_{L, R} \\
s_{L, R} \\
b_{L, R}
\end{array}\right)
$$

To generate quark masses we again substitute $\langle\Phi\rangle$ for $\Phi$, now in the first line of Eq. 2.22. We obtain mass matrices $\frac{v}{\sqrt{2}} \tilde{G}_{i j}$ and $\frac{v}{\sqrt{2}} G_{i j}$ which are diagonalized by the matrices $A$ and $B$ to yield masses $m_{u}, \ldots, m_{b}$ of $u, \ldots, b$ quarks:

$$
\frac{v}{\sqrt{2}} A_{R}^{-1} \tilde{G} A_{L}=\left(\begin{array}{ccc}
m_{u} & 0 & 0 \\
0 & m_{c} & 0 \\
0 & 0 & m_{t}
\end{array}\right), \quad \frac{v}{\sqrt{2}} B_{R}^{-1} G B_{L}=\left(\begin{array}{ccc}
m_{d} & 0 & 0 \\
0 & m_{s} & 0 \\
0 & 0 & m_{b}
\end{array}\right) .
$$

Mixings arise in the charged current interactions of quarks: the quark charged current Lagrangian (part of $\mathcal{L}_{F}$, Eq. 2.7) is given as

$$
\mathcal{L}_{c c}=\frac{g_{2}}{\sqrt{2}} W^{\mu} \overline{\left(u_{1_{L}}^{\prime}, u_{2_{L}}^{\prime}, u_{3_{L}}^{\prime}\right)} \gamma_{\mu}\left(\begin{array}{c}
d_{1_{L}}^{\prime} \\
d_{2_{L}}^{\prime} \\
d_{3_{L}}^{\prime}
\end{array}\right)=\frac{g_{2}}{\sqrt{2}} W^{\mu} \overline{\left(u_{L}, c_{L}, t_{L}\right)} A_{L}^{\dagger} B_{L} \gamma_{\mu}\left(\begin{array}{c}
d_{L} \\
s_{L} \\
b_{L}
\end{array}\right)
$$

where $V_{C K M} \equiv A_{L}^{\dagger} B_{L}$ is the $3 \times 3$ unitary CKM mixing matrix 22. It is a nondiagonal matrix inducing transitions between families in charged current interactions. Acting on mass eigenstates $d, s, b$, it gives us weak eigenstates $\tilde{d}, \tilde{s}, \tilde{b}$ (see Eq. 2.5). There is no mixing in the neutral current Lagrangian, hence no flavour-changing neutral currents at the lowest order of perturbation theory, although they can arise at the one-loop level.

For neutrino masses, there is an empty space in the second line of Eq. 2.22 
because no right-handed neutrino fields $\nu_{R}$ are included. Thus, there cannot be nonzero neutrino masses in the SM f

The generation of fermion masses in the SM, as we have just described it, is considered to be the least satisfactory part of the SM. Each mass enters as an unknown parameter (Yukawa couplings are not predicted) which has to be supplied by experiment. The SM rather accommodates fermion masses than predicts them. The problem of fermion masses, and neutrino masses in particular, has been a top priority for particle physicists for some time now.

\subsection{Neutrino masses in $S U(2)_{L} \times U(1)_{Y}$ models be- yond the SM}

As noted in the Introduction, basic directions in the theoretical treatment of neutrino masses can be followed in the class of models based on the same symmetry group as the SM, on $S U(2)_{L} \times U(1)_{Y}$. This fixes the gauge sector; the fermion content and the Higgs (symmetry breaking) sector offer some freedom which is used by different models within the class. We keep here also the symmetry breaking sector of the SM untouched and extend the fermion sector only. We examine two such models in this section and the third one, our model, in Chapter 3.

\subsubsection{A simple model of neutrino mass}

In this straightforward extension of the SM one postulates one right-handed neutrino field $\nu_{R}$ per family with the $S U(2)_{L} \times U(1)_{Y}$ quantum numbers $(0,0)$. Neutrinos are then treated in the same manner as all other fermions in the SM. The presence of

\footnotetext{
${ }^{4}$ There actually could be nonzero neutrino masses without right-handed neutrino fields if the Higgs sector of the SM was appropriately extended [15].
} 
right-handed neutrino fields allows new Yukawa interactions,

$$
\mathcal{L}_{\text {new }}=-\sum_{i=1}^{3} \sum_{j=1}^{3} \tilde{h}_{i j} \overline{\nu_{i_{R}}}\left(\tilde{\Phi}^{\dagger} \psi_{j_{L}}\right)+\text { h.c. } .
$$

This is the term missing from Eq. 2.22. Neutrinos acquire Dirac mass by analogy with up type quarks in the SM (see Sec. 2.2); the only minor difference is that here we do not introduce mixings among the charged leptons. Neutrino mass eigenstates are then different from weak eigenstates $\nu_{e}, \nu_{\mu}, \nu_{\tau}$, leading to neutrino mixing and the violation of family lepton numbers.

The shortcoming of this model is that it provides no answer to the problem of smallness of neutrino masses. We can make masses small by tuning Yukawa couplings $\tilde{h}_{i j}$ but this is not satisfactory, as there is no good reason why the $\tilde{h}_{i j}$ should themselves be small.

\subsubsection{See-saw mechanism in an $S U(2)_{L} \times U(1)_{Y}$ model, Ma- jorana neutrinos and Majorana NHL's}

Charged fermions are formally described by Dirac spinors. Neutrinos are described in the same way in the simple extension discussed above. However, because neutrinos are neutral, another possibility opens up. They could be Majorana particles. To illustrate the difference between Dirac and Majorana neutrinos, let us decompose the Dirac mass term into its components, which form the so-called Majorana basis of a matrix representation of mass terms (see Ref. [15], Sec. 4.5),

$$
\begin{aligned}
m \bar{\nu} \nu & =m\left(\overline{\nu_{L}} \nu_{R}+\overline{\nu_{R}} \nu_{L}\right)=m \overline{\nu_{L}} \nu_{R}+\text { h.c. }=\frac{1}{2} m\left(\overline{\nu_{L}} \nu_{R}+\overline{\nu_{L}^{c}} \nu_{R}^{c}\right)+\text { h.c. }= \\
& =\frac{1}{2}\left(\overline{\nu_{L}^{c}} \overline{\nu_{L}}\right)\left(\begin{array}{cc}
0 & m \\
m & 0
\end{array}\right)\left(\begin{array}{c}
\nu_{R} \\
\nu_{R}^{c}
\end{array}\right)+\text { h.c. }
\end{aligned}
$$




$$
=\frac{1}{2}\left(\overline{\nu_{L}} \overline{\nu_{L}^{c}}\right)\left(\begin{array}{cc}
0 & m \\
m & 0
\end{array}\right)\left(\begin{array}{c}
\nu_{R}^{c} \\
\nu_{R}
\end{array}\right)+\text { h.c. },
$$

where $\nu^{c}=C \gamma_{0} \nu^{*}\left(C=i \gamma^{2} \gamma^{0}\right)$ is the charge conjugate field of $\nu, \nu_{R}^{c} \equiv \frac{1}{2}\left(1+\gamma_{5}\right) \nu^{c}$ is the charge conjugate of the field $\nu_{L}$, and $\nu_{L}^{c}$ is the charge conjugate of the field $\nu_{R}$. In the above, we used the identity $\overline{\nu_{L}^{c}} \nu_{R}^{c}=\overline{\nu_{L}} \nu_{R}$, proven in Appendix A.4. From Eq. 2.29 it is obvious that the Dirac mass term has a very special mass matrix in the Majorana basis, namely, the two diagonal terms are zero. Can we make these two matrix elements nonzero ? The answer is yes, if we are willing to accept the violation of the total lepton number $L$, or equivalently baryon minus lepton $(B-L)$ number 5 . We already broke individual lepton family numbers and there is nothing sacred about $B-L$ symmetry either.

In an $S U(2)_{L} \times U(1)_{Y}$ see-saw model we introduce the following mass matrix (written for the case of one family),

$$
-\mathcal{L}_{\text {mass }}=\frac{1}{2}\left(\overline{\nu_{L}} \overline{n_{L}^{c}}\right)\left(\begin{array}{cc}
0 & D \\
D & M
\end{array}\right)\left(\begin{array}{c}
\nu_{R}^{c} \\
n_{R}
\end{array}\right)+\text { h.c. }
$$

so the fermion content is the same as that of the simple model of Sec. 2.3.1 0 , but here we allow Majorana mass terms breaking $B-L$ number conservation ฤ,

$$
\frac{1}{2} M \overline{n_{L}^{c}} n_{R}+\text { h.c. . }
$$

\footnotetext{
${ }^{5}$ The relevance of $B-L$, rather than $L$, is discussed in Ref. [15], Sec. 2.4.

${ }^{6}$ Note that in Eq. 2.29 we use notation $\nu_{L}, \nu_{R}$ for left-handed and right-handed chiral fields respectively; in contrast, here we use $n_{R}$ rather than $\nu_{R}$ for the right-handed field. The reason lies in the fact that for a Dirac neutrino two independent fields $\nu_{L}, \nu_{R}$ combine to form a single particle, while in this case $\nu_{L}$ with its partner $\nu_{R}^{c}$ form (in the limit $M \gg D$ ) a light Majorana neutrino and $n_{R}$ with its partner $n_{L}^{c}$ form a Majorana NHL; hence we use a different notation for the field describing a different particle.

${ }^{7}$ For $B-L$ to be conserved, $\mathcal{L}_{\text {mass }}$ must be invariant under the following transformations: $\nu_{L} \rightarrow e^{-i(B-L) \alpha} \nu_{L}=e^{-i \alpha} \nu_{L} ; n_{R} \rightarrow e^{-i \alpha} n_{R} ; n_{L}^{c} \rightarrow e^{+i \alpha} n_{L}^{c} ; \overline{n_{L}^{c}} \rightarrow e^{-i \alpha} \overline{n_{L}^{c}}$. The term in Eq. 2.31 transforms as $\overline{n_{L}^{c}} n_{R} \rightarrow e^{-i \alpha} e^{-i \alpha} \overline{n_{L}^{c}} n_{R} \neq \overline{n_{L}^{c}} n_{R}$, i.e., it breaks $B-L$ conservation.
} 
The matrix $\mathcal{M}=\left(\begin{array}{cc}0 & D \\ D & M\end{array}\right)$ now describes two massive Majorana neutrinos rather than a single Dirac one. To see that, we have to diagonalize $\mathcal{M}$ (e.g. Ref. [15], Sec. 5.1.4),

$$
\mathcal{M}=O^{T}\left(\begin{array}{ll}
m_{1} & 0 \\
0 & m_{2}
\end{array}\right)\left(\begin{array}{rr}
-1 & 0 \\
0 & 1
\end{array}\right) O
$$

where $m_{1,2}=\frac{1}{2}\left(\sqrt{M^{2}+4 D^{2}} \mp M\right)$ are the masses of the two Majorana neutrinos. In the above,

$$
O=\left(\begin{array}{rr}
\cos \theta & -\sin \theta \\
\sin \theta & \cos \theta
\end{array}\right), \quad \tan 2 \theta=\frac{2 D}{M}
$$

is an orthogonal rotation matrix defining massive Majorana neutrinos $\nu^{\prime}, N$ as

$$
\left(\begin{array}{c}
\nu_{L}^{\prime} \\
N_{L}
\end{array}\right) \equiv O\left(\begin{array}{c}
\nu_{L} \\
n_{L}^{c}
\end{array}\right), \quad\left(\begin{array}{c}
\nu_{R}^{\prime} \\
N_{R}
\end{array}\right) \equiv\left(\begin{array}{rr}
-1 & 0 \\
0 & 1
\end{array}\right) O\left(\begin{array}{c}
\nu_{R}^{c} \\
n_{R}
\end{array}\right)
$$

From here we can show

$$
\begin{aligned}
& \nu^{\prime}=\nu_{L}^{\prime}+\nu_{R}^{\prime}=\cos \theta\left(\nu_{L}-\nu_{R}^{c}\right)-\sin \theta\left(n_{L}^{c}-n_{R}\right)=-\nu^{\prime c}, \\
& N=N_{L}+N_{R}=\sin \theta\left(\nu_{L}+\nu_{R}^{c}\right)+\cos \theta\left(n_{L}^{c}+n_{R}\right)=N^{c},
\end{aligned}
$$

that is, $\nu^{\prime}$ and $N$ are their own charge conjugates, their own antiparticles; therefore they are Majorana neutrinos.

We see how this model explains the small neutrino masses when we assume that $M \gg D$. In this limit, the masses $m_{1}$ of $\nu^{\prime}$ and $m_{2}$ of $N$ become

$$
m_{1} \doteq \frac{D^{2}}{M}, \quad m_{2} \doteq M
$$


and using $\sin 2 \theta \doteq \tan 2 \theta \doteq 2 \theta=\frac{2 D}{M}$, we find for the weak eigenstate $\nu_{L}$

$$
\nu_{L} \doteq \nu_{L}^{\prime}+\frac{D}{M} N_{L} \doteq \nu_{L}^{\prime}
$$

Eq. 2.36 is the famous see-saw mass relation (see also Eq. 1.2), whereby a weakly interacting neutrino, $\nu_{L} \doteq \nu_{L}^{\prime}$, gets very light compared to the typical family fermion mass $D$ thanks to the very large Majorana mass $M$. Assuming $D \sim m_{\tau}$, $M$ has to be greater than about $10^{8} \mathrm{GeV}$ in order to meet the cosmological bound (see Sec. 1.2) $m_{\nu}<25 \mathrm{eV}$.

It looks as though we have replaced the problem of the smallness of the neutrino mass with another one, the problem of the big mass $M$. Indeed, in the context of an $S U(2)_{L} \times U(1)_{Y}$ model, the origin of the big mass $M$ is a mystery. At this point we invoke our motivational grounds, the unification models (see the next section). There are in fact large scales in these models associated with the unification energies. The $S U(2)_{L} \times U(1)_{Y}$ see-saw model could be a low-energy limit of some GUT theory.

In this thesis we are specifically interested in NHL's, described in this section by the field $N$ with the mass $m_{2} \equiv M$. From Eqs. 2.36, 2.37 it is obvious that NHL's in this model are, first, too heavy to be observed directly in the near future, and second, their contribution to left-handed weak eigenstates is so small that there is little hope to see even their indirect effects. See-saw models tend to be phenomenologically uninteresting. There are however models with special forms of the Dirac and Majorana mass matrices (in the general case of $n$ families, masses $D$ and $M$ in Eq. 2.30 become Dirac and Majorana $n \times n$ mass matrices) that avoid this suppression [19, 20]. For instance, Pilaftsis [19] finds a relation among the elements of $D$ and $M$ matrices that leads to massless neutrinos at the tree-level and small Majorana masses are generated radiatively. The cosmological constraint on the scale $M$ is much weaker in this model and consequently, the mixing of NHL's $\left(K_{H} \sim D / M\right)$ is not suppressed. We shall refer to such models as see-saw models with enhanced 
mixings. Although calculations in this work were carried out in the context of a superstring-inspired model, our analysis is qualitatively valid also for this class of see-saw models.

\subsection{Neutrino mass in grand unified models}

Here we briefly touch the question of neutrino mass in grand unified models (GUT's). A nice short review of the subject can be found in Ref. [15; the case of $S O(10)$ is discussed also in Ref. [9], and that of $E_{6}$ in Ref. [23].

There are 15 chiral fermion fields per generation currently known, $e_{L}, e_{R}, \nu_{e}$ and twelve $u$ and $d$ quark fields. In the simplest GUT model, SU(5), these 15 fields are assigned to $\{10\}$ and $\overline{\{5\}}$-dimensional representations. There is no right-handed neutrino postulated; therefore, one cannot generate a Dirac mass for the neutrino and also it is not possible to generate Majorana mass as described in Sec. 2.3.2. One can still generate Majorana mass without a right-handed neutrino if an appropriate Higgs field is introduced. The problem is that this Higgs field is introduced ad hoc and, as a result, neutrino masses do not arise in $S U(5)$ naturally. Moreover, $S U(5)$ is ruled out by the proton decay measurements [24].

The next popular group is $S O(10)$. This group contains left-right symmetric $S U(2)_{L} \times S U(2)_{R} \times S U(4)_{C}$ as its subgroup, which implies automatically the righthanded neutrino. The number of chiral fermion fields per generation is thus 16, filling the fundamental $\{16\}$ representation. With a right-handed neutrino in the fundamental representation, neutrino masses in $S O(10)$ can arise naturally via the see-saw mechanism (see Sec. 2.3.2). The actual values of neutrino masses are sensitive to the Majorana mass matrix $M$ (see Eq. 2.30), which in turn can tell us about the particular branch of the $S O(10)$ breaking down to low-energy $S U(3)_{C} \times S U(2)_{L} \times U(1)_{Y}$. $S O(10)$ predicts a lower rate for proton decay than does $S U(5)$.

Finally, a lot of attention is paid to $E_{6}$ based GUT's [23]. This is thanks to their 
superstring connections. Green and Schwarz 25] showed that string theory in ten dimensions is anomaly free for the gauge group $E_{8} \times E_{8}^{\prime}$ and that the compactification of the additional six dimensions can result in the breaking of $E_{8}$ down to $E_{6}$, which becomes an effective GUT group.

The fundamental representation of $E_{6}$ is $\{27\}$-dimensional, implying 27 chiral fermion fields per generation, 11 more than we had in $S O(10)$. These eleven fields must be new particles, often referred to as exotics. They include a colour triplet weak isosinglet quark and its antiparticle and five new leptons. Of the new leptons, four (two charged and two neutral) form two weak isodoublets and the fifth one is a weak isosinglet.

Curiously, the superstring-inspired $E_{6}$ model experiences certain difficulties in understanding the small neutrino masses [17, 26] : there are no appropriate Higgs fields to provide the large Majorana mass $M$ for the see-saw mass matrix (see Eq. 2.30) and therefore the see-saw mechanism does not operate here. Interesting solutions to this problem suggest that besides the fundamental $\{27\}$-plet there exists an additional, $E_{6}$ singlet neutral fermion field $S_{L}$. At low energies, $S_{L}$, along with the right-handed neutrino $n_{R}$, can enrich the neutral lepton spectrum of the SM. The other three neutral exotic leptons decouple from the low-energy spectrum. The mass matrix formed by $\nu_{L}, n_{R}$ and $S_{L}$ offers an alternative to the see-saw mechanism in generating naturally light (in fact massless) neutrinos.

The phenomenological implications of such a superstring-inspired low-energy model, which is just a minimal extension of the SM, are studied in this thesis. The model itself is described in detail in the next chapter. 


\section{Chapter 3}

\section{A superstring-inspired $S U(2)_{L} \times U(1)_{Y}$ model of neutrino}

\section{mass}

In this thesis we study phenomenological aspects of an $S U(2)_{L} \times U(1)_{Y}$ model, which extends the neutral fermion spectrum of the SM by two new fields, the right-handed neutrino $n_{R}$ and a left-handed field $S_{L}$. The model could arise as a low-energy limit of a superstring-inspired $E_{6}$ GUT [17, 26]; it was also suggested as a low-energy limit of a supersymmetry-inspired $S O(10)$ GUT [27]. Superstring-inspired GUT's have an interesting problem with neutrino masses (see the discussion in Sec. 2.4): the seesaw mechanism does not apply here and unacceptably large neutrino masses arise as a consequence [17, 26]. The existence of the field $S_{L}$ was suggested as a potential solution to this problem. $S_{L}$ is an $E_{6}$ singlet which may be present in superstring models. At low energies it can remain in the neutral fermion spectrum along with the right-handed neutrino $n_{R}$ and the usual left-handed neutrino $\nu_{L}$. These three fields together with imposed $B-L$ conservation form a mass matrix leading to an alternative to the see-saw mechanism in addressing the problem of the smallness of 
neutrino masses.

In this chapter we define the model and give a detailed treatment of neutrino masses and mixing matrix, and the neutrino interaction Lagrangian.

\subsection{Fermion content and mass matrix}

In this superstring-inspired model we keep, in line with introductory arguments in Sec. 2.3, the gauge sector and the Higgs sector of the SM untouched. The fermion content is enlarged by two neutrino fields, $n_{R}$ and $S_{L}$, per family. Their $S U(2)_{L} \times U(1)_{Y}$ quantum numbers are $(0,0)$. The field $n_{R}$ is a right-handed neutrino, while $S_{L}$ is an $E_{6}$ singlet neutrino field. In a single family, we thus have the following leptons (given with their quantum numbers):

$$
\begin{aligned}
& \left(\begin{array}{c}
\nu_{e} \\
e
\end{array}\right)_{L} \begin{array}{ccc}
e_{R} & n_{R} & S_{L} \\
\left(\frac{1}{2},-1\right) & (0,-2) & (0,0) \quad(0,0)
\end{array}
\end{aligned}
$$

The definition of the model is completed by specifying the mass matrix $\mathcal{M}$. In the Majorana basis it is given by

$$
-\mathcal{L}_{\text {mass }}=\frac{1}{2} \mathcal{M}=\frac{1}{2}\left(\overline{\nu_{L}} \overline{n_{L}^{c}} \overline{S_{L}}\right)\left(\begin{array}{lll}
0 & D & 0 \\
D^{T} & 0 & M^{T} \\
0 & M & 0
\end{array}\right)\left(\begin{array}{c}
\nu_{R}^{c} \\
n_{R} \\
S_{R}^{c}
\end{array}\right)+h . c . .
$$

Each $\nu_{L}, n_{R}, S_{L}$ represents now a collection of three fields, one for each family, e.g. $\nu_{L}=\left(\nu_{e}, \nu_{\mu}, \nu_{\tau}\right)$ is the vector of the three SM weak eigenstate neutrinos. $D$ and $M$ are $3 \times 3$ Dirac mass matrices. The top diagonal element must vanish unless we extend the symmetry breaking sector of our model. A weak isotriplet Higgs field 
could allow this term. However, we retain the symmetry breaking sector of the SM. The middle element is zero due to the absence of the appropriate Higgs fields that would provide the Majorana mass. This is enforced by imposed $B-L$ number conservation, which is also responsible for all other zeros in the mass matrix $\mathcal{M}$. Only terms preserving the $B-L$ number, $\overline{\nu_{L}} D n_{R}+$ h.c. and $\overline{S_{L}} M n_{R}+$ h.c., remain (see footnote 7 , p. 23 on $B-L$ conservation).

To find the physical neutrino states of the model, we have to diagonalize the mass matrix $\mathcal{M}$. We will do it within a single family first and then we will generalize the procedure for the three families.

\subsubsection{Diagonalization of $\mathcal{M}$ for a single family}

In the case of a single family, $\nu_{L}, n_{R}, S_{L}$ represent each only one field and matrices $D, M$ become simple numbers. We perform the following rotation,

$$
\left(\begin{array}{c}
\nu_{L} \\
n_{L}^{c} \\
S_{L}
\end{array}\right) \equiv O\left(\begin{array}{c}
\nu_{L}^{\prime} \\
n_{L}^{c} \\
S_{L}^{\prime}
\end{array}\right), \quad\left(\begin{array}{c}
\nu_{R}^{c} \\
n_{R} \\
S_{R}^{c}
\end{array}\right) \equiv O\left(\begin{array}{c}
\nu_{R}^{\prime c} \\
n_{R} \\
S_{R}^{\prime} c
\end{array}\right)
$$

where

$$
O=\left(\begin{array}{ccc}
c_{\theta} & 0 & s_{\theta} \\
0 & 1 & 0 \\
-s_{\theta} & 0 & c_{\theta}
\end{array}\right), \quad c_{\theta}=\cos \theta, s_{\theta}=\sin \theta, \tan \theta=\frac{D}{M}
$$

The mass matrix $\mathcal{M}$ becomes

$$
\mathcal{M}=\left(\overline{\nu_{L}^{\prime}} \overline{n_{L}^{c}} \overline{S_{L}^{\prime}}\right) O^{T}\left(\begin{array}{ccc}
0 & D & 0 \\
D & 0 & M \\
0 & M & 0
\end{array}\right) O\left(\begin{array}{c}
\nu_{R}^{\prime c} \\
n_{R} \\
S_{R}^{\prime c}
\end{array}\right)+\text { h.c. }
$$




$$
\begin{aligned}
& =\left(\overline{\nu_{L}^{\prime}} \overline{n_{L}^{c}} \overline{S_{L}^{\prime}}\right)\left(\begin{array}{ccc}
0 & D c_{\theta}-M s_{\theta} & 0 \\
D c_{\theta}-M s_{\theta} & 0 & D s_{\theta}+M c_{\theta} \\
0 & D s_{\theta}+M c_{\theta} & 0
\end{array}\right)\left(\begin{array}{c}
\nu_{R}^{\prime c} \\
n_{R} \\
S_{R}^{\prime c}
\end{array}\right)+h . c . \\
& =\left(\overline{\nu_{L}^{\prime}} \overline{n_{L}^{c}} \overline{S_{L}^{\prime}}\right)\left(\begin{array}{ccc}
0 & 0 & 0 \\
0 & 0 & \sqrt{D^{2}+M^{2}} \\
0 & \sqrt{D^{2}+M^{2}} & 0
\end{array}\right)\left(\begin{array}{c}
\nu_{R}^{\prime c} \\
n_{R} \\
S_{R}^{\prime c}
\end{array}\right)+h . c .
\end{aligned}
$$

yielding a massless neutrino $\nu^{\prime}$. Moreover, we recognize the submatrix

$$
\left(\overline{n_{L}^{c}} \overline{S_{L}^{\prime}}\right)\left(\begin{array}{cc}
0 & \sqrt{D^{2}+M^{2}} \\
\sqrt{D^{2}+M^{2}} & 0
\end{array}\right)\left(\begin{array}{c}
n_{R} \\
S_{R}^{\prime c}
\end{array}\right)
$$

as the matrix representation of a Dirac mass term (see Eq. 2.29) in the Majorana basis. Indeed, putting

$$
n_{L}^{c} \equiv N_{L}^{c}, \quad S_{R}^{c} \equiv N_{R}^{c}, \quad n_{R} \equiv N_{R}, \quad S_{L}^{\prime} \equiv N_{L}
$$

we reproduce Eq. 2.29 and therefore, besides the massless neutrino $\nu^{\prime}$, we generate a Dirac neutral heavy lepton $N$ with the mass $M^{\prime}=\sqrt{D^{2}+M^{2}}$. The weak eigenstate $\nu_{L}$ is given by

$$
\begin{aligned}
\nu_{L} & =\cos \theta \nu_{L}^{\prime}+\sin \theta S_{L}^{\prime} \\
& =\frac{M}{\sqrt{D^{2}+M^{2}}} \nu_{L}^{\prime}+\frac{D}{\sqrt{D^{2}+M^{2}}} N_{L} \\
& \equiv K_{L} \nu_{L}^{\prime}+K_{H} N_{L},
\end{aligned}
$$

where $K_{L}, K_{H}$ are mixing factors (matrices in the case of three families) for massless neutrinos and NHL's, respectively.

For $M \gg D$ the mass of the NHL, $M^{\prime}$, and the weak eigenstate $\nu_{L}$ are approxi- 
mately

$$
\begin{aligned}
M^{\prime} & \doteq M \\
\nu_{L} & \doteq \nu_{L}^{\prime}+\frac{D}{M} N_{L}
\end{aligned}
$$

that is, the mixing of NHL's is $K_{H} \doteq \frac{D}{M}$.

\subsubsection{Mass matrix diagonalization in case of three families}

We leave the matrix representation of the mass matrix observing that

$$
\begin{aligned}
-\mathcal{L}_{\text {mass }} & =\frac{1}{2} \mathcal{M}=\frac{1}{2}\left(\overline{\nu_{L}} \overline{n_{L}^{c}} \overline{S_{L}}\right)\left(\begin{array}{ccc}
0 & D & 0 \\
D^{T} & 0 & M^{T} \\
0 & M & 0
\end{array}\right)\left(\begin{array}{c}
\nu_{R}^{c} \\
n_{R} \\
S_{R}^{c}
\end{array}\right)+\text { h.c. } \\
& =\frac{1}{2}\left(\overline{\nu_{L}} D n_{R}+\overline{n_{L}^{c}} D^{T} \nu_{R}^{c}+\overline{n_{L}^{c}} M^{T} S_{R}^{c}+\overline{S_{L}} M n_{R}\right)+\text { h.c. } \\
& =\frac{1}{2}\left(\overline{\nu_{L}} D n_{R}+\overline{\nu_{L}} D n_{R}+\overline{S_{L}} M n_{R}+\overline{S_{L}} M n_{R}\right)+\text { h.c. } \\
& =\overline{\nu_{L}} D n_{R}+\overline{S_{L}} M n_{R}+\text { h.c. . }
\end{aligned}
$$

In the above we used the identity (for which the proof is almost identical with that for $\overline{\nu_{L}^{c}} \nu_{R}^{c}=\overline{\nu_{L}} \nu_{R}$, see Appendix A.4)

$$
\overline{n_{L}^{c}} D^{T} \nu_{R}^{c}=\overline{\nu_{L}} D n_{R}
$$

Performing the following rotation

$$
\left(\begin{array}{c}
\nu_{L}^{\prime} \\
S_{L}^{\prime}
\end{array}\right)=G\left(\begin{array}{c}
\nu_{L} \\
S_{L}
\end{array}\right)=\left(\begin{array}{cc}
U_{1} & U_{2} \\
U_{3} & U_{4}
\end{array}\right)\left(\begin{array}{c}
\nu_{L} \\
S_{L}
\end{array}\right)
$$


where $G$ is a unitary matrix, we get

$$
\begin{aligned}
-\mathcal{L}_{\text {mass }} & =\left(\overline{\nu_{L}^{\prime}} U_{1}+\overline{S_{L}^{\prime}} U_{3}\right) D n_{R}+\left(\overline{\nu_{L}^{\prime}} U_{2}+\overline{S_{L}^{\prime}} U_{4}\right) M n_{R}+\text { h.c. } \\
& =\overline{\nu_{L}^{\prime}}\left(U_{1} D+U_{2} M\right) n_{R}+\text { h.c. }+\overline{S_{L}^{\prime}}\left(U_{3} D+U_{4} M\right) n_{R}+\text { h.c. } .
\end{aligned}
$$

We put (compare with $D \cos \theta-M \sin \theta=0$ for a single generation case)

$$
U_{1} D+U_{2} M=0
$$

hence

$$
-\mathcal{L}_{\text {mass }}=\overline{S_{L}^{\prime}}\left(U_{3} D+U_{4} M\right) n_{R}+\text { h.c. }=\overline{S_{L}^{\prime}} M^{\prime} n_{R}+\text { h.c. }
$$

There is no mass term for $\nu_{L}^{\prime}$, therefore $\nu_{L}^{\prime}$ is a massless neutrino. $M^{\prime}$, unlike in the one family case, has to be further diagonalized with rotations ( $Z, T$ unitary matrices) in the NHL basis:

$$
S_{L}^{\prime \prime}=T S_{L}^{\prime}, \quad n_{R}^{\prime \prime}=Z n_{R}
$$

yielding

$$
\begin{aligned}
-\mathcal{L}_{\text {mass }} & =\overline{S_{L}^{\prime \prime}}\left(T U_{3} D Z^{\dagger}+T U_{4} M Z^{\dagger}\right) n_{R}^{\prime \prime}+\text { h.c. } \\
& =\overline{S_{L}^{\prime \prime}} M^{\prime \prime} n_{R}^{\prime \prime}+\text { h.c. }
\end{aligned}
$$

such that $M^{\prime \prime}$ is diagonal. Identifying (see also Eq. 3.7)

$$
\begin{aligned}
S_{L}^{\prime \prime} & \equiv N_{L}, \\
n_{R}^{\prime \prime} & \equiv N_{R},
\end{aligned}
$$


we arrive at

$$
-\mathcal{L}_{\text {mass }}=\bar{N} M^{\prime \prime} N=M_{N_{4}} \overline{N_{4}} N_{4}+M_{N_{5}} \overline{N_{5}} N_{5}+M_{N_{6}} \overline{N_{6}} N_{6}
$$

Here $M^{\prime \prime}$ is diagonal with elements $M_{N_{4}}, M_{N_{5}}, M_{N_{6}}$ being masses of three Dirac NHL's $N_{4}, N_{5}, N_{6}$. The weak eigenstate vector, $\nu_{L}$, is given by

$$
\begin{aligned}
\nu_{L} \equiv \nu_{l} \equiv\left(\nu_{e}, \nu_{\mu}, \nu_{\tau}\right) & =U_{1}^{\dagger} \nu_{L}^{\prime}+U_{3}^{\dagger} S_{L}^{\prime}=U_{1}^{\dagger} \nu_{L}^{\prime}+U_{3}^{\dagger} T^{\dagger} S_{L}^{\prime \prime} \\
& \equiv K_{L} \nu_{L}^{\prime}+K_{H} S_{L}^{\prime \prime}=K_{L} \nu_{L}^{\prime}+K_{H} N_{L} .
\end{aligned}
$$

\subsubsection{Discussion of mass eigenstates}

The diagonalization of the mass matrix yielded three massless neutrinos $\nu_{L}^{\prime} \equiv$ $\left(\nu_{1_{L}}^{\prime}, \nu_{2_{L}}^{\prime}, \nu_{3_{L}}^{\prime}\right)$ along with three Dirac NHL's $N \equiv\left(N_{4}, N_{5}, N_{6}\right)$ with mass $M_{N} \sim M$. The masslessness of the neutrinos is the consequence of the assumed $B-L$ symmetry. This symmetry also prevents neutrinos from acquiring small masses in radiative corrections. The neutrinos are massless due to $B-L$ symmetry also in the SM, but the difference is that in the SM the $B-L$ symmetry is an automatic consequence of the missing right-handed neutrino fields, while here this symmetry is imposed with right-handed neutrinos present. Note that massless neutrinos imply there are no time dependent neutrino oscillations and no neutrinoless double beta decays.

In the light of arguments for massive neutrinos (see Sec. 1.2) it may seem surprising that this model yields massless neutrinos. However, small neutrino masses can be generated in a variant of our model by introducing a small Majorana mass term $\mu[17,18]$ in the mass matrix $\mathcal{M}$ in Eq. 3.2 :

$$
-\mathcal{L}_{\text {mass }}=\frac{1}{2} \mathcal{M}=\frac{1}{2}\left(\overline{\nu_{L}} \overline{n_{L}^{c}} \overline{S_{L}}\right)\left(\begin{array}{lll}
0 & D & 0 \\
D^{T} & 0 & M^{T} \\
0 & M & \mu
\end{array}\right)\left(\begin{array}{c}
\nu_{R}^{c} \\
n_{R} \\
S_{R}^{c}
\end{array}\right)+\text { h.c. }
$$


Besides, now that we have a superstring motivation for the field content of our model, there is nothing unusual about massless neutrinos either. The unnaturalness of the SM treatment of neutrino masses is removed, dark matter has other candidates and the solar neutrino puzzle has alternative explanations. Whether neutrinos have any mass at all may actually be of secondary interest for a theorist who is trying to come up with some plausible explanation of the low experimental limits on this mass.

The weak eigenstates in our model $\left(\nu_{L}\right)$ are dominated by massless neutrinos $\nu_{L}^{\prime}$ with a small admixture $\left(K_{H} \sim D / M\right.$, see Eq. 3.9) of NHL's $N$. In see-saw models, the NHL mixing is generally suppressed due to the scale $M$ (see Eq. 2.36 and the discussion afterwards) ${ }^{1}$, which has to be very large to explain small neutrino masses, $m_{\nu} \sim \frac{D^{2}}{M}$, dictated by experiments and cosmological arguments. The NHL mixing in our model is not, however, restricted by the dependence of neutrino masses $m_{\nu^{\prime}}$ on scales $D$ and $M$ (there is no such dependence as $m_{\nu^{\prime}}=0$ ). Therefore, the scale $M$ can be much lower than in the case of see-saw models and hence rates for many interesting phenomena can be large. This means that signatures of NHL's might be found even at current accelerator energies and luminosities. Our model is thus attractive not only conceptually, but also practically.

\subsection{Properties of the mixing matrix}

The weak interaction eigenstates $\nu_{l}$ are related to six mass eigenstates $\nu^{\prime}, N$ via a $3 \times 6$ mixing matrix $K$ with components $K_{l \alpha} ; l=e, \mu, \tau$ and $\alpha=\nu_{1}^{\prime}, \nu_{2}^{\prime}, \nu_{3}^{\prime}, N_{4}, N_{5}, N_{6}$ (see Eq. 3.20)

$$
\left(\begin{array}{c}
\nu_{e} \\
\nu_{\mu} \\
\nu_{\tau}
\end{array}\right)=\left(\begin{array}{llllll}
K_{e \nu_{1}^{\prime}} & K_{e \nu_{2}^{\prime}} & K_{e \nu_{3}^{\prime}} & K_{e N_{4}} & K_{e N_{5}} & K_{e N_{6}} \\
K_{\mu \nu_{1}^{\prime}} & K_{\mu \nu_{2}^{\prime}} & K_{\mu \nu_{3}^{\prime}} & K_{\mu N_{4}} & K_{\mu N_{5}} & K_{\mu N_{6}} \\
K_{\tau \nu_{1}^{\prime}} & K_{\tau \nu_{2}^{\prime}} & K_{\tau \nu_{3}^{\prime}} & K_{\tau N_{4}} & K_{\tau N_{5}} & K_{\tau N_{6}}
\end{array}\right)\left(\begin{array}{c}
\prime \\
\nu_{L}^{\prime} \\
N_{L}
\end{array}\right)
$$

\footnotetext{
${ }^{1}$ An exception are the see-saw models with enhanced mixings discussed at the end of Sec. 2.3.2.
} 


$$
\equiv\left(\begin{array}{ll}
K_{L} K_{H}
\end{array}\right)\left(\begin{array}{c}
\nu_{L}^{\prime} \\
N_{L}
\end{array}\right) ; \quad \nu^{\prime}=\left(\begin{array}{c}
\nu_{1}^{\prime} \\
\nu_{2}^{\prime} \\
\nu_{3}^{\prime}
\end{array}\right), \quad N=\left(\begin{array}{c}
N_{4} \\
N_{5} \\
N_{6}
\end{array}\right)
$$

Alternatively, we can write 7

$$
\nu_{l}=\sum_{i=1,2,3}\left(K_{L}\right)_{l i} \nu_{i_{L}}^{\prime}+\sum_{a=4,5,6}\left(K_{H}\right)_{l a} N_{a_{L}}=\left(K_{L}\right)_{l i} \nu_{i_{L}}^{\prime}+\left(K_{H}\right)_{l a} N_{a_{L}} .
$$

A quick inspection tells us the matrix $K$ has $3 \times 6$ complex parameters $=36$ degrees of freedom. Unitarity implies an important property often used throughout this work,

$$
K_{L} K_{L}^{\dagger}+K_{H} K_{H}^{\dagger}=1
$$

This property reduces the number of degrees of freedom by 9 to 27 . Further elimination of unphysical parameters via redefinition (rephasing) 5 of physical mass eigenstates leaves us $3^{2}$ angles and $(3-1)^{2}$ phases [29]. This allows for possible lepton flavour violation, universality violation and $\mathrm{CP}$ violation.

The mixing factor which typically governs flavour-conserving processes, $l l_{m i x}$, is given by

$$
l l_{m i x}=\sum_{a=4,5,6}\left(K_{H}\right)_{l a}\left(K_{H}^{\dagger}\right)_{a l} ; \quad l=e, \mu, \tau
$$

and the flavour-violating mixing factor $l l^{\prime}$ mix is defined as

$$
l l_{\text {mix }}^{\prime}=\sum_{a=4,5,6}\left(K_{H}\right)_{l a}\left(K_{H}^{\dagger}\right)_{a l^{\prime}} ; \quad l, l^{\prime}=e, \mu, \tau, \quad l \neq l^{\prime} .
$$

\footnotetext{
${ }^{2}$ Where not indicated in this work, indices $i, j, k$ run through $1,2,3$ and $a, b, c$ through $4,5,6$.

${ }^{3}$ This operation is also done in the SM when one parametrizes the CKM matrix.
} 
Further, the following important inequality holds

$$
\left|l l_{\text {mix }}^{\prime}\right|^{2} \leq l l_{\text {mix }} l^{\prime} l_{\text {mix }}^{\prime}, \quad l \neq l^{\prime}
$$

This implies that one might observe nonstandard effects in flavour-conserving processes even if they are absent in flavour-violating processes.

\subsection{Interaction Lagrangians}

The charged and neutral current Lagrangians are obtained from the corresponding terms in the SM Lagrangian substituting for $\nu_{l}$ from Eq. 3.23. The charged current Lagrangian is given by

$$
\begin{aligned}
\mathcal{L}_{c c} & =\frac{1}{2 \sqrt{2}} g_{2} W^{\mu} \sum_{l=e, \mu, \tau}\left\{\sum_{i} \bar{l} \gamma_{\mu}\left(1-\gamma_{5}\right)\left(K_{L}\right)_{l i} \nu_{i}^{\prime}+\sum_{a} \bar{l} \gamma_{\mu}\left(1-\gamma_{5}\right)\right. \\
& \left.\times\left(K_{H}\right)_{l a} N_{a}\right\}+ \text { h.c. }
\end{aligned}
$$

and the neutral current Lagrangian as

$$
\begin{aligned}
\mathcal{L}_{n c} & =\frac{g_{2}}{4 c_{W}} Z^{\mu} \sum_{i, a} \bar{\nu}_{i}^{\prime}\left(K_{L}^{\dagger} K_{H}\right)_{i a} \gamma_{\mu}\left(1-\gamma_{5}\right) N_{a}+\text { h.c. } \\
& +\frac{g_{2}}{4 c_{W}} Z^{\mu} \sum_{a, b} \bar{N}_{a}\left(K_{H}^{\dagger} K_{H}\right)_{a b} \gamma_{\mu}\left(1-\gamma_{5}\right) N_{b} \\
& +\frac{g_{2}}{4 c_{W}} Z^{\mu} \sum_{i, j} \bar{\nu}_{i}^{\prime}\left(K_{L}^{\dagger} K_{L}\right)_{i j} \gamma_{\mu}\left(1-\gamma_{5}\right) \nu_{j}^{\prime} .
\end{aligned}
$$

We will also need Lagrangians with neutrinos and NHL's interacting with the Higgs $H$ and with unphysical Higgs $\phi^{+}, \phi^{-}$and $\chi$. The starting point is the Yukawa Lagrangian describing the interactions of neutrino fields $\nu_{l}, n_{R}$ with the Higgs dou- 
blet $\tilde{\Phi}$

$$
\mathcal{L}=-\left(\overline{\nu_{l}} \overline{l_{L}}\right) \tilde{h} \tilde{\Phi} n_{R}+h . c .
$$

where $\tilde{h}$ is a matrix (in family space) of Yukawa couplings. The $S_{L}$ field does not couple to $\tilde{\Phi}$, but rather might couple to a new $S U(2)_{L} \times U(1)_{Y}$ singlet Higgs field (responsible also for mass $M$ ) present in some superstring models [26]. We do not introduce such a field.

The physical Higgs part, derived in Appendix B, is given by

$$
\begin{aligned}
\mathcal{L}_{H} & =-\frac{g_{2}}{2 M_{W}} \bar{N}\left(K_{H}^{\dagger} K_{H}\right) M_{N} N H \\
& -\frac{g_{2}}{2 M_{W}} \overline{\nu^{\prime}}\left(K_{L}^{\dagger} K_{H}\right) M_{N} \frac{1+\gamma_{5}}{2} N H \\
& -\frac{g_{2}}{2 M_{W}} \bar{N}\left(K_{H}^{\dagger} K_{L}\right) M_{N} \frac{1-\gamma_{5}}{2} \nu^{\prime} H
\end{aligned}
$$

the unphysical neutral Higgs $\chi$ part,

$$
\begin{aligned}
\mathcal{L}_{\chi} & =+i \frac{g_{2}}{2 M_{W}} \bar{N}\left(K_{H}^{\dagger} K_{H}\right) M_{N} \gamma_{5} N \chi \\
& +i \frac{g_{2}}{2 M_{W}} \overline{\nu^{\prime}}\left(K_{L}^{\dagger} K_{H}\right) M_{N} \frac{1+\gamma_{5}}{2} N \chi \\
& -i \frac{g_{2}}{2 M_{W}} \bar{N}\left(K_{H}^{\dagger} K_{L}\right) M_{N} \frac{1-\gamma_{5}}{2} \nu^{\prime} \chi
\end{aligned}
$$

and the unphysical charged Higgs $\phi^{+}, \phi^{-}$parts

$$
\mathcal{L}_{\phi^{-}}=+\frac{g_{2}}{\sqrt{2} M_{W}} \overline{e_{L}} K_{H} M_{N} N_{R} \phi^{-}+O\left(\frac{m_{l}}{M_{W}}\right)+\text { h.c. } .
$$

Feynman rules corresponding to these Lagrangians are listed in Appendix $\mathbf{9}$. 


\subsection{Review of existing constraints on NHL's}

Constraints on neutral heavy lepton masses and mixings come from three different sources.

i) First, there is the possibility of direct production of NHL's. At $e^{+} e^{-}$colliders such as LEP I or SLC, they could be produced in Z decays: f

$$
Z \rightarrow N_{a}+\nu
$$

and subsequently decay via neutral or charged currents:
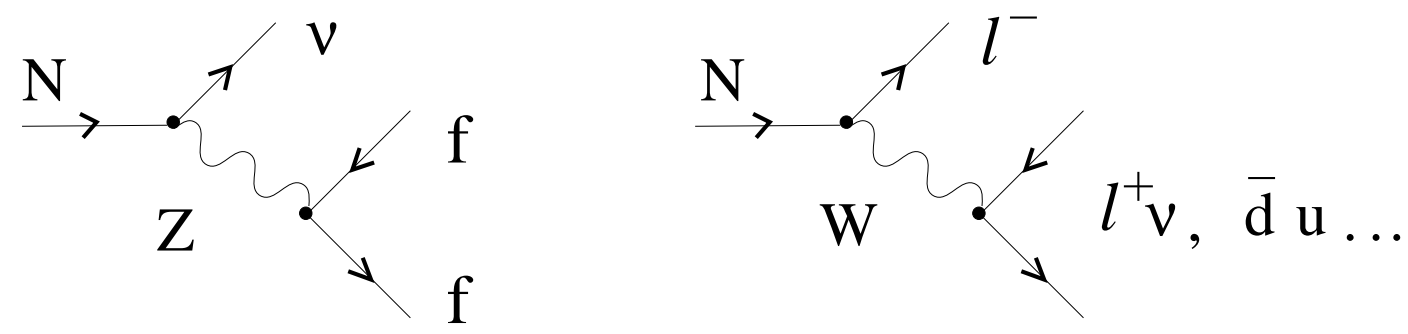

The rate for $Z$ decays into an NHL and a light neutrino has been given previously [30] as

$$
\Gamma\left(Z \rightarrow N_{a}+\nu\right)=a_{m i x}\left(1-\frac{M_{N_{a}}{ }^{2}}{M_{Z}{ }^{2}}\right)\left(1+\frac{M_{N_{a}}{ }^{2}}{2 M_{Z}{ }^{2}}\right) \Gamma(Z \rightarrow \nu+\nu),
$$

where

$$
a_{m i x}=\sum_{l=e, \mu, \tau}\left|\left(K_{H}\right)_{l a}\right|^{2}
$$

\footnotetext{
${ }^{4}$ In the rest of this work we drop the prime from $\nu^{\prime}$.
} 
The subsequent NHL decay rate (for $M_{N} \leq M_{W}$ ) is then given by

$$
\Gamma_{N}=a_{m i x}\left(\frac{M_{N}}{m_{\mu}}\right)^{5} \Phi_{l} \Gamma_{\mu},
$$

where $\Gamma_{\mu}$ is the muon decay rate and $\Phi_{l}$ is the effective number of decay channels available to the NHL [31]. LEP data effectively (better than indirect constraints, see below) probe NHL mixings for NHL mass up to $80 \mathrm{GeV}$ [30] .

NHL production at $p p$ supercolliders was studied in Ref. [32]. It was concluded that the CERN Large Hadron Collider (LHC) has the potential to push the limits on $a_{m i x}$ below the LEP constraints for NHL mass up to $110 \mathrm{GeV}$.

ii) Second, there are constraints on NHL mixing parameters from a variety of low energy experiments and from experiments at LEP I where neutral heavy leptons are not directly present. NHL's however, do affect observables indirectly: due to unitarity properties of the mixing matrix $K$, a nonzero NHL mixing slightly reduces the couplings of light neutrinos from their SM values ?, thus affecting rates for nuclear $\beta$ decays, $\tau$ and $\pi$ decays, and for $Z$ decays. The following upper limits are consistent with experiment [33]

$$
\begin{aligned}
e e_{\text {mix }} & \leq 0.0071 \\
\mu \mu_{\text {mix }} & \leq 0.0014 \\
\tau \tau_{\text {mix }} & \leq 0.033
\end{aligned}
$$

The limit on $\tau \tau_{m i x}$ is improved to $\leq 0.024$ if the invisible width of the $\mathrm{Z}$ boson is included in the analysis [33]. The limits in Eq. 3.38 are model independent and hold for any value of the NHL mass. They arise from a global analysis of results including lepton universality experiments, CKM matrix unitarity tests, $W$ mass

\footnotetext{
${ }^{5}$ For example in case of $W e \nu$ vertex, the mixing is changed from $\mathrm{SM}$ value $=1$ to $K_{L}$, see Eq. 3.28 .
} 
measurements and neutral current data from LEP I experiments. Note that the LEP I neutral current data analysis did not include NHL loop effects but, rather, only coupling constant modifications due to mixing. We consider NHL loop effects in this work.

Since the limit on the parameter $\tau \tau_{m i x}$ plays (as the least stringent one) the most important role in our analysis, we will pay further attention to its source. It comes from the $\mu-\tau$ universality test based on the $\tau$ leptonic decays compared to the $\mu$ leptonic decays. The result of the test is given as the ratio of the couplings of $\tau$ and $\mu$ to the $\mathrm{W}$ boson, $g_{\tau} / g_{\mu}$ (in the SM we have $g_{\tau}=g_{\mu}=g_{2}$ ). The ratio is found from

$$
\frac{\Gamma(\tau \rightarrow e \nu \nu) / \Gamma^{S M}(\tau \rightarrow e \nu \nu)}{\Gamma(\mu \rightarrow e \nu \nu) / \Gamma^{S M}(\mu \rightarrow e \nu \nu)}=\left(\frac{g_{\tau}}{g_{\mu}}\right)^{2}=\frac{1-\tau \tau_{m i x}}{1-\mu \mu_{m i x}}
$$

Setting $\mu \mu_{\text {mix }}=0$, we get

$$
\tau \tau_{m i x}=1-\left(\frac{g_{\tau}}{g_{\mu}}\right)^{2}
$$

with 33

$$
\left(\frac{g_{\tau}}{g_{\mu}}\right)^{2}=0.989 \pm 0.016
$$

iii) Finally, the NHL masses and mixings can be constrained via their contribution in loops to various processes. The calculation to the one-loop level of the perturbation theory is naturally more involved than the mostly tree-level considerations required for direct and indirect constraints. In return we can probe regions in the mixings vs NHL mass parameter space currently inaccessible to the direct and indirect methods. For example, as we will see, we can place upper limits on the NHL mass. We caution though that these limits depend on the mixings and they will be relaxed should tighter bounds on mixings be achieved. Still this is an improvement over the direct and indirect methods which are blind to NHL masses larger than 


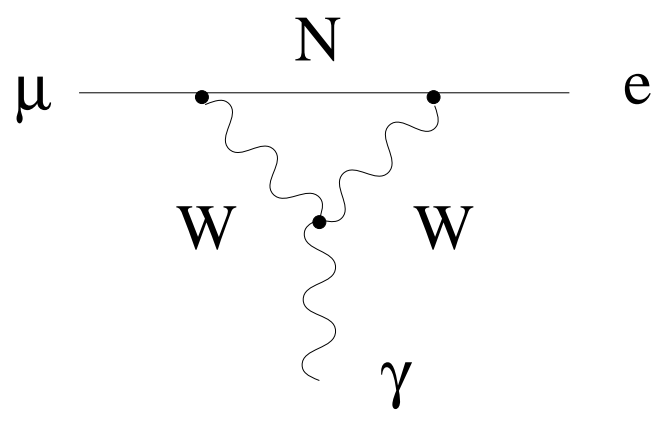

Figure 3.1: A one-loop diagram leading to $\mu \rightarrow e \gamma$ decay

$M_{Z}$

There are two classes of these processes, lepton flavour-violating and lepton flavour-conserving. Lepton flavour-violating decays have been considered a hot candidate for a new physics manifestation in general for many decades. They include so far unobserved, so-called rare decays of $\mu$ and $\tau$ leptons and $\mu-e$ conversion in nuclei $(\mathrm{A}, \mathrm{Z})$ :

$$
\begin{gathered}
\mu \rightarrow e \gamma, \quad \tau \rightarrow e \gamma, \quad \tau \rightarrow \mu \gamma, \\
\mu, \tau \rightarrow e e^{+} e^{-}, \quad \tau \rightarrow \mu e^{+} e^{-}, \quad \tau \rightarrow e \mu^{+} \mu^{-}, \quad \tau \rightarrow \mu \mu^{+} \mu^{-}, \\
\mu^{-}(A, Z) \rightarrow e^{-}(A, Z),
\end{gathered}
$$

and the $\mathrm{Z}$ boson decays

$$
Z \rightarrow e^{ \pm} \mu^{\mp}, \quad Z \rightarrow e^{ \pm} \tau^{\mp}, \quad Z \rightarrow \mu^{ \pm} \tau^{\mp} .
$$

We will discuss these decays in Chapter 5 ; here we at least mention the decay $\mu \rightarrow e \gamma$, which underwent an intensive experimental scrutiny and its stringent upper limit places a tough constraint on the mixing parameter $e \mu_{m i x}$. For illustration, one of the diagrams contributing to $\mu \rightarrow e \gamma$ is shown in Fig. 3.1. In Sec. 5.2 we will show 
that $\mu \rightarrow e \gamma$ gives the following upper limit on the mixing parameter $e \mu_{m i x}$ :

$$
\left|e \mu_{\text {mix }}\right| \leq 0.00024
$$

By combining the indirect constraints obtained from the global analysis (see Eq. 3.38) with the inequality relations of Eq. 3.27 one obtains the following upper limits on the mixing factors

$$
\begin{aligned}
& \left|e \mu_{\text {mix }}\right| \leq 0.0032 \\
& \left|\mu \tau_{\text {mix }}\right| \leq 0.0068 \\
& \left|e \tau_{\text {mix }}\right| \leq 0.015
\end{aligned}
$$

For the mixings $\mu \tau_{\text {mix }}$ and $e \tau_{\text {mix }}$, these are the strongest available constraints.

The second class consists of lepton flavour-conserving processes with NHL's in loops. The main part of this thesis (Chapters 6 and 7 ) is devoted to two of these processes, $Z \rightarrow l^{+} l^{-}$with partial leptonic width $\Gamma_{l l}$ and universality breaking parameter $U_{b r}$ as observables ; and $\mu \rightarrow e \nu_{e} \nu_{\mu}$ with the $\mathrm{W}$ mass $M_{W}$ as observable. We will argue that the flavour-conserving processes can be competitive with and even have some advantages over the flavour-violating ones. 


\section{Chapter 4}

\section{Standard model at the one-loop level}

As a prerequisite for one-loop calculations in Chapters 5,6 and 7, we discuss here the standard model of electroweak interactions at the one-loop level. The classical electroweak Lagrangian was specified in Sec. 2.1. One-loop corrections require treatment within the framework of the quantum field theory: the classical Lagrangian has to be quantized and extended to include some new terms. We present these quantum field theoretical 'amendments' to the classical Lagrangian in Sec. 4.1.

One-loop corrections calculated from the full Lagrangian typically suffer from divergences. A systematic way of removing these divergences, the renormalization of the SM, is discussed in Sec. 4.2. There are a lot of different schemes used to renormalize the SM. We opted for the on-shell renormalization scheme of W. Hollik [34, 35], introduced in Sec. 4.3. 


\subsection{Quantization}

The quantum field theory requires two more terms to be added to the classical Lagrangian, $\mathcal{L}_{\text {gfix }}$ and $\mathcal{L}_{\text {ghost }}$ :

$$
\mathcal{L}_{E W}=\mathcal{L}_{G}+\mathcal{L}_{F}+\mathcal{L}_{H}+\mathcal{L}_{\text {gfix }}+\mathcal{L}_{\text {ghost }}
$$

The gauge fixing term $\mathcal{L}_{g f i x}$ is required in order to define meaningful propagators of the gauge fields which are otherwise singular [36. The linear gauge fixing of the 't Hooft type is given by 34

$$
\mathcal{L}_{g f i x}=-\frac{1}{2}\left(F_{\gamma}^{2}+F_{Z}^{2}+2 F_{+} F_{-}\right)
$$

where

$$
\begin{aligned}
& F_{ \pm}=\frac{1}{\sqrt{\xi^{W}}}\left(\partial^{\mu} W_{\mu}^{ \pm} \mp i M_{W} \xi^{W} \phi^{ \pm}\right) \\
& F_{Z}=\frac{1}{\sqrt{\xi^{Z}}}\left(\partial^{\mu} Z_{\mu}-M_{Z} \xi^{Z} \chi\right) \\
& F_{\gamma}=\frac{1}{\sqrt{\xi^{\gamma}}} \partial^{\mu} A_{\mu}
\end{aligned}
$$

and $\xi^{W}, \xi^{Z}, \xi^{\gamma}$ are gauge fixing parameters. In the 't Hooft type gauge the vector boson propagators have the form $(V=W, Z)$

$$
\frac{i}{k^{2}-M_{V}^{2}+i \epsilon}\left(-g^{\mu \nu}+\frac{\left(1-\xi^{V}\right) k^{\mu} k^{\nu}}{k^{2}-\xi^{V} M_{V}^{2}+i \epsilon}\right),
$$

and propagators of unphysical Higgs particles $\phi^{ \pm}, \chi$ are given by

$$
\frac{i}{k^{2}-\xi^{V} M_{V}^{2}+i \epsilon}
$$


The unitary gauge is defined by $\xi^{V} \rightarrow \infty$. We can see that in this gauge unphysical Higgs freeze out (their propagators vanish) and only physical particles appear in Feynman diagrams.

In this work we use the Feynman gauge defined by $\xi^{V}=\xi^{\gamma}=1$. In this gauge there are unphysical Higgs present, but the positive trade-off is the particularly simple form of the gauge boson propagators of Eq. 4.4.

The $\mathcal{L}_{\text {ghost }}$ term [36, 34, 37] is specific to nonabelian theories where the oneloop self-energies of the gauge bosons computed from $\mathcal{L}_{G}+\mathcal{L}_{F}+\mathcal{L}_{H}+\mathcal{L}_{\text {gfix }}$ do not satisfy gauge invariance and unitarity. $\mathcal{L}_{\text {ghost }}$ removes this difficulty with scalar anticommuting ghost fields $u^{ \pm}, u^{Z}, u^{\gamma}$ (Fadeev-Popov ghosts) which appear naturally in Fadeev-Popov quantization based on the path-integral method [38. $u^{ \pm}, u^{Z}$ propagators are the same as the propagators of unphysical Higgs, Eq. 4.5, while the $u^{\gamma}$ propagator is given by

$$
\frac{i}{k^{2}+i \epsilon}
$$

\subsection{Renormalization}

In $\mathcal{L}_{E W}$ there are five independent parameters (showing only lepton Yukawa couplings $h_{i}$ and counting them as one):

$$
g_{2}, g_{1}, \lambda, v, h_{i}
$$

After symmetry breaking we can replace them by an equivalent set (counting $m_{f}$ as one)

$$
\text { e, } M_{W}, M_{Z}, M_{H}, m_{f}
$$


where $e^{2} / 4 \pi=\alpha$ and masses are those of $W, Z$ and Higgs bosons and of a fermion, respectively.

Originally these parameters were identified with their physical values $(\alpha=$ $1 / 137, M_{Z}=91.137 \mathrm{GeV}$, etc.). However, loop corrections calculated in terms of the physical values of these parameters diverge and the parameters themselves are modified - their physical values are changed by an infinite amount. Renormalization takes care of these (so-called ultraviolet) infinities through the reexamination of the meaning of the Lagrangian parameters in Eq. 4.7. We will illustrate the process on the electric charge $e$. In an effort to get to the core of the one-loop renormalization of the SM, we present at times simplified versions of the SM formulae. The reader is made aware of the simplifications in a series of footnotes.

\subsubsection{Electric charge renormalization}

The piece of $\mathcal{L}_{E W}$ defining the electric charge is the interaction term of the QED Lagrangian

$$
\mathcal{L}_{e m}=e \bar{l} \gamma_{\mu} l A^{\mu}
$$

We identify $\alpha=e^{2} / 4 \pi$ with its physical value $1 / 137$ measured in low-energy (Thomson limit $k^{2}=(p-q)^{2} \rightarrow 0$, see diagram below) electron scattering. The photon - charged lepton vertex corresponding to $\mathcal{L}_{e m}$ is given by

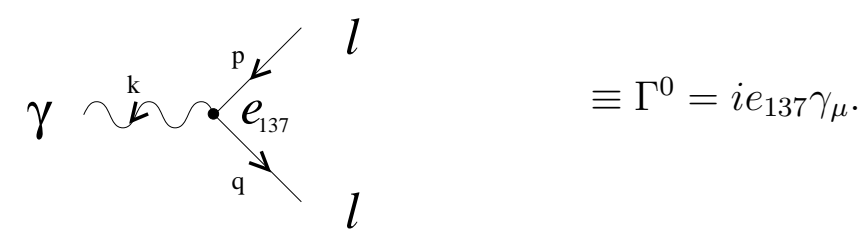

\footnotetext{
${ }^{1}$ In this section we enforce $\alpha=1 / 137.036$ using notation $\alpha_{137}, e_{137}$
} 
One-loop corrections to this vertex, calculated in terms of $e_{137}$, are $\mathrm{f}$
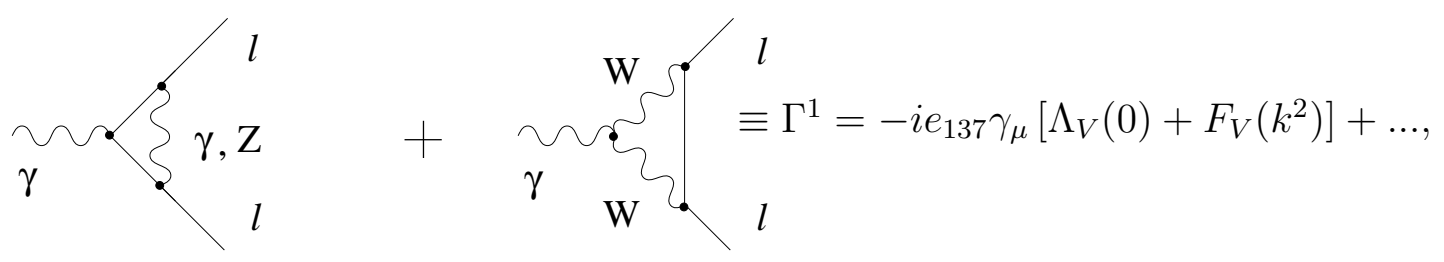

where ellipses $\ldots$ represent terms with Lorentz structure different from $\gamma_{\mu}$ s, $F_{V}$ is the form factor split off so that $F_{V}(0)=0$ and $\Lambda_{V}(0)$ is given by

$$
\Lambda_{V}(0)=-\frac{\alpha_{137}}{4 \pi}\left(\frac{2}{\epsilon}+\text { finite constants }\right)
$$

where $2 / \epsilon$ is the ultraviolet divergence $(\epsilon \rightarrow 0)$ regularized by dimensional regularization (see Appendix D on dimensional regularization). The one-loop corrected vertex $\Gamma$ is thus given by

$$
\Gamma=\Gamma^{0}+\Gamma^{1}=i e_{137} \gamma_{\mu}\left[1-\Lambda_{V}(0)-F_{V}\left(k^{2}\right)\right]+\ldots
$$

At low energies (Thomson limit $k^{2} \rightarrow 0$ )

$$
\Gamma\left(k^{2} \rightarrow 0\right)=i e_{137} \gamma_{\mu}\left[1-\Lambda_{V}(0)\right]+\ldots,
$$

\footnotetext{
${ }^{2}$ Strictly speaking, lepton self-energies, $\gamma$ self-energy and $\gamma-Z$ mixing also contribute to this vertex at the one-loop level. They are, however, each renormalized independently (in the on-shell scheme, for example, they vanish in the Thomson limit) and they will not change the essence of our arguments.

${ }^{3} \gamma_{\mu} \gamma_{5}$ and $(p+q)_{\mu}$, renormalized independently

${ }^{4}$ This form is exact only for the diagram with $\gamma$ in the loop, the other two diagrams have the divergence multiplied by some combinations of $s_{W}^{2}$. There is also an infrared divergence present in the former diagram that we do not deal with here.
} 
hence the charge is changed from its tree-level value in $\Gamma^{0}$ by an infinite amount:

$$
e_{137} \rightarrow e_{137}\left[1-\Lambda_{V}(0)\right]
$$

To explain this difficulty, we note the quantity measured by the experiment as $e_{137}$ is a loop corrected charge. But the quantity on the right-hand side of Eq. 4.12 is a loop (one-loop) corrected charge, therefore the quantity multiplying the $1-\Lambda_{V}(0)$ factor, denoted $e_{137}$, cannot be $e_{137}$. This implies the charge in the electromagnetic Lagrangian cannot be identified with its physical value. The correct approach is to admit the independent parameters appearing in $\mathcal{L}_{E W}$ are in fact 'bare', unrenormalized quantities

$$
e^{b}, M_{W}^{b}, M_{Z}^{b}, M_{H}^{b}, m_{f}^{b}
$$

different from the physical values. The vertex $\Gamma$ is then given by (compare with Eq. 4.10)

$$
\begin{aligned}
\Gamma & =\Gamma^{0}+\Gamma^{1}=i e^{b} \gamma_{\mu}\left[1-\Lambda_{V}(0)-F_{V}\left(k^{2}\right)\right]+\ldots \\
& =i e^{b} \gamma_{\mu}\left[1+\frac{\alpha^{b}}{4 \pi}\left(\frac{2}{\epsilon}+\text { finite constants }\right)-F_{V}\left(k^{2}\right)\right]+\ldots
\end{aligned}
$$

Bare parameters are unambiguously fixed by the requirement that they lead to correct physical values. For electric charge we demand that (in view of the discussion above)

$$
\Gamma\left(k^{2} \rightarrow 0\right)=i e^{b} \gamma_{\mu}\left[1-\Lambda_{V}(0)\right]+\ldots=i e_{137} \gamma_{\mu}
$$

or equivalently

$$
e^{b}\left[1-\Lambda_{V}(0)\right]=e_{137} .
$$


From here (after plugging in $\Lambda_{V}(0)$ calculated in terms of $\alpha^{b}$ ) we can solve for $e^{b}$, 向

$$
e^{b}=e_{137}\left[1-\frac{\alpha_{137}}{4 \pi}\left(\frac{2}{\epsilon}+\text { finite constants }\right)\right]
$$

Using Eqs. 4.16, 4.17, the Eq. 4.14 can be written as

$$
\Gamma=i e_{137} \gamma_{\mu}\left[1-F_{V}\left(k^{2}\right)\right]+\ldots
$$

where $F_{V}\left(k^{2}\right)$ is of the order $O\left(\alpha_{137}\right)$. The infinity is thus removed from the vertex $\Gamma$, i.e., the electromagnetic vertex (and the charge) is renormalized.

\subsubsection{Renormalization schemes}

The loop calculations are rarely carried out in terms of bare parameters. A widely used technique is to split the bare charge $e^{b}$, the bare fermion mass $m_{f}^{b}$ and the bare boson mass $M^{b}$ as

$$
\begin{aligned}
e^{b} & =\hat{e}+\delta e \\
m_{f}^{b} & =\hat{m}_{f}+\delta m_{f}, \\
M_{b}^{2} & =\hat{M}^{2}+\delta M^{2}
\end{aligned}
$$

where $\hat{e}, \hat{m}_{f}, \hat{M}$ are renormalized (finite) charge, fermion, and gauge boson masses and $\delta e, \delta m_{f}, \delta M^{2}$ are infinite corrections, so-called counterterms. This split introduces a degree of freedom, as there is no unique way to perform it. Renormalized charge and mass can take on different finite values including the physical ones. This freedom leads in practice to many different ways of splitting the bare parameters, i.e., to many different renormalization schemes (RS).

The difference between two renormalized charges coming from two different RS

\footnotetext{
${ }^{5}$ We work to order $O\left(e_{137} \alpha_{137}\right)$ in Sec. 4.2. Higher order terms are neglected.
} 
is small, of the order $O\left(\alpha_{137}\right)$, as every renormalized charge is chosen to be equal to $e_{137}$ in the lowest order:

$$
\hat{e}=e_{137}\left[1+O\left(\alpha_{137}\right)\right]
$$

With the substitution of Eq. 4.19, the $\mathcal{L}_{\text {em }}$ becomes

$$
\mathcal{L}_{e m}=e^{b} \bar{l} \gamma_{\mu} l A^{\mu}=\hat{e} \bar{l} \gamma_{\mu} l A^{\mu}+\delta e \bar{l} \gamma_{\mu} l A^{\mu}
$$

where the second term is called the counterterm Lagrangian. The calculation of the vertex $\Gamma$ now leads to

$$
\hat{\Gamma}=\Gamma^{0}+\Gamma^{1}=i \hat{e} \gamma_{\mu}\left[1-\Lambda_{V}(0)+\delta e / \hat{e}-F_{V}\left(k^{2}\right)\right]+\ldots
$$

The conditions of Eqs. 4.15, 4.16 are now given by

$$
\begin{aligned}
\hat{\Gamma}\left(k^{2} \rightarrow 0\right)=i \hat{e} \gamma_{\mu}\left[1-\Lambda_{V}(0)+\delta e / \hat{e}\right]+\ldots & =i e_{137} \gamma_{\mu} \\
\hat{e}\left[1-\Lambda_{V}(0)+\delta e / \hat{e}\right] & =e_{137}
\end{aligned}
$$

At this point we can illustrate two different approaches to the choice of renormalization scheme.

i) If we prefer to use some particular value of $\hat{e}$ in the calculation, say

$$
\hat{e}=e_{137}\left[1+b \alpha_{137}\right]
$$

\footnotetext{
${ }^{6}$ Note $\delta e / \hat{e}$ is of the order $O(\hat{\alpha})=O\left(\alpha_{137}\right)$.

${ }^{7}$ From now on we will use $\Gamma=i \hat{e} \gamma_{\mu}\left[1-\Lambda_{V}(0)-F_{V}\left(k^{2}\right)\right]$ for the unrenormalized vertex, and $\hat{\Gamma}$ for the renormalized vertex, $\hat{\Gamma}=\Gamma+$ counterterm. The counterterm contains besides $\delta e$ also wave function renormalization factors, not considered until Sec. 4.2.4.
} 
the counterterm $\delta Z \equiv \delta e / \hat{e}$ is consequently fixed by (see Eq. 4.24)

$$
\delta Z \equiv \delta e / \hat{e}=\frac{e_{137}}{\hat{e}}+\Lambda_{V}(0)-1
$$

In fact, the most popular scheme in electroweak calculations is an on-shell scheme (OS) where

$$
\begin{aligned}
\hat{e} \equiv \hat{e}^{O S} & =e_{137} \quad(b=0) \\
\delta Z^{O S} \equiv \delta e / \hat{e}^{O S} & =\Lambda_{V}(0)=-\frac{\alpha_{137}}{4 \pi}\left(\frac{2}{\epsilon}+\text { finite constants }\right),
\end{aligned}
$$

and all masses assume their physical, on-shell values $\left(\hat{M}_{Z}^{O S}=M_{Z}=91.1884 \mathrm{GeV}\right.$, etc.). The Eq. 4.23 with $\hat{e}=e_{137}$,

$$
\hat{\Gamma}\left(k^{2} \rightarrow 0\right)=i e_{137} \gamma_{\mu}
$$

is called an on-shell renormalization condition.

ii) A different approach is to start with fixing the counterterm $\delta e$ instead of $\hat{e}$. For instance we may require that $\delta e\left(\delta m_{f}, \delta M^{2}\right.$ likewise) only contain infinities (no finite terms) 9 . This is the essence of minimal subtraction (MS) scheme. One chooses

$$
\delta Z^{M S} \equiv \delta e / \hat{e}^{M S}=-\frac{\hat{\alpha}^{M S}}{4 \pi} \frac{2}{\epsilon} \doteq-\frac{\alpha_{137}}{4 \pi} \frac{2}{\epsilon}
$$

and the charge is consequently given by

$$
\hat{e} \equiv \hat{e}^{M S}=\frac{e_{137}}{\left[1-\Lambda_{V}(0)+\delta Z^{M S}\right]} \doteq \frac{e_{137}}{1+\frac{\alpha_{137}}{4 \pi} \times \text { finite terms }}
$$

hence the electric charge $\hat{e}^{M S}$ differs from $e_{137}$ and likewise masses $\hat{m}_{f}{ }^{M S}, \hat{M}^{M S}$ do not assume their on-shell values. The MS scheme is frequently used in quantum

\footnotetext{
${ }^{8}$ Compare this with OS where $\delta e / \hat{e}^{O S}=\Lambda_{V}(0)$ contains both infinite and finite terms.
} 
chromodynamics where on-shell quark masses are not well defined anyway.

\subsubsection{Mass renormalization}

The analysis performed above for the electric charge is essentially valid also for masses. The difference is in the form of renormalization condition. Masses can be defined as the poles of the propagators. For instance, gauge boson propagators $V=W, Z$ have poles at bare mass $M_{V}^{b}$ :

$$
\equiv P^{0}=\frac{-i g^{\mu \nu}}{k^{2}-M_{V}^{b^{2}}+i \epsilon}
$$

The one-loop correction to $P^{0}$ is given by

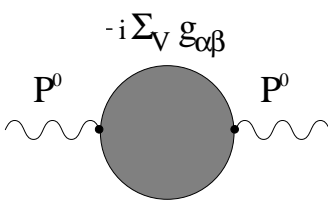

$$
\equiv P^{1}=\frac{-i g^{\mu \alpha}}{k^{2}-M_{V}^{b}+i \epsilon}\left(-i \Sigma_{V} g_{\alpha \beta}\right) \frac{-i g^{\beta \nu}}{k^{2}-M_{V}^{b}+i \epsilon}
$$

For a close-up of the blob $-i \Sigma_{V} g_{\alpha \beta}$ (unrenormalized vector boson self-energy tensor), see Figs. 6.4, 6.5 and the relevant discussion in Chapter 6. The one-loop corrected renormalized ${ }^{9}$ propagator is thus (compare with Eq. 4.22)

$$
P=P^{0}+P^{1}=\frac{-i g^{\mu \nu}}{k^{2}-M_{V}^{b^{2}}+i \epsilon}\left[1-\frac{\Sigma_{V}}{k^{2}-M_{V}^{b}+i \epsilon}\right]
$$

\footnotetext{
${ }^{9}$ Here, we mean renormalized as far as mass is concerned. There is still one divergence remaining in $\Sigma_{V}$ which will be removed only after the field renormalization, see Sec. 4.2.4. Therefore we will withhold the notation $\hat{P}$ until then.
} 


$$
\begin{aligned}
& \cong \frac{-i g^{\mu \nu}}{k^{2}-M_{V}^{b}+i \epsilon} \frac{1}{\left(1+\frac{\Sigma_{V}}{k^{2}-M_{V}^{b}+i \epsilon}\right)}=\frac{-i g^{\mu \nu}}{k^{2}-M_{V}^{b}+\Sigma_{V}\left(k^{2}\right)+i \epsilon} \\
& =\frac{-i g^{\mu \nu}}{k^{2}-\hat{M}_{V}^{2}-\delta M_{V}^{2}+\Sigma_{V}\left(k^{2}\right)+i \epsilon} .
\end{aligned}
$$

We demand that the poles (masses) of renormalized propagators remain at their physical values regardless of the choice of $\hat{M}_{V}$ or $\delta M_{V}^{2}$ (compare with Eq. 4.23):

$$
P\left(k^{2} \rightarrow M_{V}^{2}\right)=\frac{-i g^{\mu \nu}}{k^{2}-\hat{M}_{V}^{2}-\delta M_{V}^{2}+\Sigma_{V}\left(M_{V}^{2}\right)+i \epsilon}=\frac{-i g^{\mu \nu}}{k^{2}-\hat{M}_{V}^{O S^{2}}+i \epsilon}
$$

To put the mass on shell we have to take

$$
\hat{M}_{V}=\hat{M}_{V}^{O S} \equiv M_{V}
$$

or equivalently

$$
\Sigma_{V}\left(M_{V}^{2}\right)-\delta M_{V}^{2}=0
$$

\subsubsection{Field renormalization}

For physical S-matrix elements, the renormalization of the five parameters in Eq. 4.7 is all that is required. However, if one wishes to also have finite Green functions, then the renormalization of the fields is also required (see, e.g., Ref. [34]).

While the particle masses are given by the poles of the propagators, the normalization of the fields is given by the residues of the propagators. For gauge boson fields, for example, we have:

$$
\frac{-i g^{\mu \nu}}{k^{2}-M_{V}^{2}+i \epsilon}
$$

with the residue equal to one (ignoring $-i g^{\mu \nu}$ ). The residue (field normalization) is 
changed by the loop corrections. To show that, we expand $\Sigma_{V}\left(k^{2}\right)$ about $k^{2}=M_{V}^{2}$ :

$$
\begin{aligned}
\Sigma_{V}\left(k^{2}\right)= & \Sigma_{V}\left(M_{V}^{2}\right)+\left(k^{2}-M_{V}^{2}\right) \Sigma_{V}^{\prime}\left(M_{V}^{2}\right)+\ldots \\
& \Sigma_{V}^{\prime} \equiv \partial \Sigma_{V} / \partial k^{2}
\end{aligned}
$$

and substitute it into the propagator of Eq. 4.31:

$$
P=\frac{-i g^{\mu \nu}}{k^{2}-\hat{M}_{V}^{2}-\delta M_{V}^{2}+\Sigma_{V}\left(M_{V}^{2}\right)+\left(k^{2}-M_{V}^{2}\right) \Sigma_{V}^{\prime}\left(M_{V}^{2}\right)+\ldots+i \epsilon} .
$$

Applying the on-shell condition Eqs. 4.33, 4.34 we get

$$
\begin{aligned}
P & =\frac{-i g^{\mu \nu}}{k^{2}-M_{V}^{2}+\left(k^{2}-M_{V}^{2}\right) \Sigma_{V}^{\prime}\left(M_{V}^{2}\right)+\ldots+i \epsilon} \\
& \cong \frac{-i g^{\mu \nu}}{\left(k^{2}-M_{V}^{2}+i \epsilon\right)} \frac{1}{\left[1+\Sigma_{V}^{\prime}\left(M_{V}^{2}\right)+\ldots\right]}
\end{aligned}
$$

with the (divergent) residue $1 /\left[1+\Sigma_{V}^{\prime}\left(M_{V}^{2}\right)\right]$ at $k^{2} \rightarrow M_{V}^{2}$. The problem is fixed by the field counterterms generated by the substitution

$$
V_{\mu} \rightarrow Z_{V}^{1 / 2} V_{\mu}=\left(1+\delta Z_{V}\right)^{1 / 2} V_{\mu} \doteq\left(1+\frac{1}{2} \delta Z_{V}\right) V_{\mu}
$$

The field counterterm $\delta Z_{V}$ modifies the propagator as follows

$$
\hat{P}=\frac{1}{1+\delta Z_{V}} P=\frac{1}{\left(1+\delta Z_{V}\right)} \frac{-i g^{\mu \nu}}{\left(k^{2}-M_{V}^{2}+i \epsilon\right)} \frac{1}{\left[1+\Sigma_{V}^{\prime}\left(M_{V}^{2}\right)\right]}
$$

To renormalize the fields (enforce their normalization) we demand that the residues 10

$$
\begin{aligned}
M_{V}^{2} V_{\mu} V^{\mu} & \rightarrow\left(1+\delta Z_{V}\right) M_{V}^{2} V_{\mu} V^{\mu}, \\
\left(\square+M_{V}^{2}\right) V_{\mu} & \rightarrow\left(1+\delta Z_{V}\right)\left(\square+M_{V}^{2}\right) V_{\mu} .
\end{aligned}
$$


of the field propagators be equal to one at the poles. This implies the following condition (higher order term neglected):

$$
\Sigma_{V}^{\prime}\left(M_{V}^{2}\right)+\delta Z_{V}=0 .
$$

It is easy to see from Eqs. 4.31, 4.40 that before being put on shell, $\hat{P}$ in terms of the renormalized self energy $\hat{\Sigma}_{V}$ is given by

$$
\begin{aligned}
\hat{P} & =\frac{-i g^{\mu \nu}}{k^{2}-\hat{M}_{V}^{2}+\hat{\Sigma}_{V}\left(k^{2}\right)+i \epsilon}, \\
\hat{\Sigma}_{V}\left(k^{2}\right) & =\Sigma_{V}\left(k^{2}\right)-\delta M_{V}^{2}+\delta Z_{V}\left(k^{2}-\hat{M}_{V}^{2}\right),
\end{aligned}
$$

so that the on-shell renormalization conditions Eqs. 4.34, 1.41 become

$$
\begin{aligned}
\hat{\Sigma}_{V}\left(M_{V}^{2}\right) & =0, \\
\frac{\partial \hat{\Sigma}_{V}\left(M_{V}^{2}\right)}{\partial k^{2}} & =0 .
\end{aligned}
$$

Before we go further, one remark is in order. So far we have been discussing a simplified renormalization of some parameters at the one-loop level. In the next section we will stay at one-loop level, however, we will present the full set of counterterms and OS renormalization conditions required for the processes studied in this thesis.

To prove the renormalizability of the SM, it has to be shown that the infinities one encounters in loop calculations to any order can be removed by the finite number of counterterms. This was done by 't Hooft in Refs. [39, 40] for a general case of non-abelian theories with spontaneous symmetry breaking. 


\subsection{The on-shell scheme of W. Hollik}

There are many renormalization schemes used in the calculation of loop corrections by different authors. They are distinguished in the first place by the choice of independent input parameters. The choice $e, M_{W}, M_{Z}, M_{H}, m_{f}$ that we are using is only one of several possible. Given the set of input parameters, there are still infinitely many possibilities for choosing the renormalized quantities $\hat{e}, \hat{m}$. The OS scheme is the most popular and natural in the standard model of electroweak interactions.

Even then, within the OS itself, there are many different approaches to renormalization. For instance, some opt for field renormalization, others do not and those who do, do it with different numbers of field renormalization constants.

In this thesis we follow the OS scheme $\left(e, M_{W}, M_{Z}, M_{H}, m_{f}\right)$ of Wolfgang Hollik [34, 35]. We introduce multiplicative renormalization constants for each free parameter and each symmetry multiplet of fields ${ }^{11}$ at the level of the unbroken theory:

$$
\begin{aligned}
W_{\mu}^{a} & \rightarrow\left(Z_{2}^{W}\right)^{\frac{1}{2}} W_{\mu}^{a} \\
B_{\mu} & \rightarrow\left(Z_{2}^{B}\right)^{\frac{1}{2}} B_{\mu} \\
\psi_{j_{L}} & \rightarrow\left(Z_{L}^{j}\right)^{\frac{1}{2}} \psi_{j_{L}} \\
\psi_{j_{R}} & \rightarrow\left(Z_{R}^{j}\right)^{\frac{1}{2}} \psi_{j_{R}} \\
\Phi & \rightarrow\left(Z^{\Phi}\right)^{\frac{1}{2}} \Phi \\
g_{2} & \rightarrow Z_{1}^{W}\left(Z_{2}^{W}\right)^{-\frac{3}{2}} g_{2} \\
g_{1} & \rightarrow Z_{1}^{B}\left(Z_{2}^{B}\right)^{-\frac{3}{2}} g_{1}
\end{aligned}
$$

\footnotetext{
${ }^{11}$ Multiplicative renormalization and only one constant per multiplet guarantees the gauge invariance of the counterterm Lagrangian. To make a connection with Eq. 4.19, note that

$$
\hat{e} \rightarrow Z \hat{e}=(1+\delta Z) \hat{e}=\hat{e}+\delta e .
$$
}




$$
\begin{aligned}
v & \rightarrow\left(Z^{\Phi}\right)^{\frac{1}{2}}(v-\delta v) \\
\lambda & \rightarrow Z^{\lambda}\left(Z^{\Phi}\right)^{-2} \lambda \\
h_{j} & \rightarrow\left(Z^{\Phi}\right)^{-\frac{1}{2}} Z_{1}^{j} h_{j},
\end{aligned}
$$

ten constants in all (counting Yukawa couplings as one). Five of them are associated with fields and five with coupling constants.

To generate the counterterm Lagrangian $\delta \mathcal{L}$, the renormalization constants are expanded as

$$
Z=1+\delta Z
$$

and Eqs. 4.46 - 4.47 are applied to $\mathcal{L}_{E W}$. The counterterms added to the unrenormalized quantities then yield the renormalized self-energies given in Appendix $\mathbb{E}$, Eq. E.2; and the renormalized electromagnetic, weak neutral and charged current vertices given in Eq. E.3. These renormalized expressions can be compared with Eq. 4.22 and Eq. 4.43.

The ten independent counterterm constants are fixed by the nine on-shell renormalization conditions $\square$. The first set of conditions puts the masses on-shell (compare with Eq. 4.44) 13:

$$
\hat{\Sigma}^{W}\left(M_{W}^{2}\right)=\hat{\Sigma}^{Z}\left(M_{Z}^{2}\right)=\hat{\Sigma}^{H}\left(M_{H}^{2}\right)=\hat{\Sigma}^{f}\left(m_{f}^{2}\right)=0
$$

where $\hat{\Sigma}^{W}, \hat{\Sigma}^{Z}, \hat{\Sigma}^{H}$ and $\hat{\Sigma}^{f}$ are the $W, Z$, Higgs and fermion renormalized self-energies respectively; the second set of conditions is the generalization of the QED electric

\footnotetext{
${ }^{12}$ The condition on $\hat{\Sigma}^{f}(k)$ in Eq. 4.49 below fixes both $\delta Z_{L}$ and $\delta Z_{R}$ constants.

${ }^{13}$ Only real parts of self-energies enter these conditions. The imaginary parts are finite.
} 
charge renormalization:

$$
\begin{aligned}
\hat{\Gamma}^{\gamma e e}\left(k^{2} \rightarrow 0\right) \equiv \hat{\Gamma}\left(k^{2} \rightarrow 0\right) & =i e \gamma_{\mu} \\
\hat{\Sigma}^{\gamma Z}\left(k^{2} \rightarrow 0\right) & =0 \\
{\left.\left[\frac{\partial}{\partial k^{2}} \hat{\Sigma}^{\gamma}\left(k^{2}\right)\right]\right|_{k^{2}=0} } & =0 \\
\left.\frac{1}{\not k-m_{f}} \hat{\Sigma}^{f}(k)\right|_{\not k=m_{f}} & =0 \\
{\left.\left[\frac{\partial}{\partial k^{2}} \hat{\Sigma}^{H}\left(k^{2}\right)\right]\right|_{k^{2}=M_{H}^{2}} } & =0
\end{aligned}
$$

where $\hat{\Sigma}^{\gamma}$ and $\hat{\Sigma}^{\gamma Z}$ are renormalized photon self-energy and photon-Z mixing respectively. The conditions involving $\hat{\Sigma}^{f}, \hat{\Gamma}^{\gamma e e}$ and $\hat{\Sigma}^{\gamma}$ come directly from QED. Derivative conditions can be compared with Eq. 4.45 derived for $\mathrm{W}$ and $\mathrm{Z}$ bosons.

When writing down renormalization conditions, one has to be careful not to violate Ward (Slavnov - Taylor) identities [41]. These consequences of gauge symmetry also relate renormalization constants to one another and can be used as a cross-check of the consistency of the renormalization conditions. In the set above, for example, Ward identities make the axial part of $\hat{\Gamma}^{\gamma e e}$ vanish in the Thomson limit [34, 35].

The renormalization constants calculated from Eqs. 4.48, 4.49 are given in Appendix E, Eq. E.4. 


\section{Chapter 5}

\section{Lepton flavour-violating processes}

Among the processes with NHL's in the loops, the lepton flavour-violating decays have so far received a lot more attention [20, 26, 42, 43, 44, 45, 46, 47, 48] than the flavour-conserving processes [49, 50]. One of the reasons could be a certain preconception that the experimental signature of the flavour violation is 'much more dramatic'. It is our intention to show in this and the next chapter that in many cases this expectation is rather naive.

Another probable reason (this time justified) is that the calculation of the flavourviolating processes is simpler, with the smaller number of contributing diagrams and without having to actually renormalize. We will demonstrate this in Sec. 5.1 in case of the flavour-violating decays of the $\mathrm{Z}$ boson. These rare processes were studied in the context of our model previously [26, 46]; however, the limit of large NHL mass was not fully investigated. This was pointed out in Ref. [47], where the branching ratios for $Z \rightarrow l_{1}^{-} l_{2}^{+}\left(e^{ \pm} \mu^{\mp}, \mu^{ \pm} \tau^{\mp}, e^{ \pm} \tau^{\mp}\right)$ were derived in the see-saw model of Ref. [19]. We therefore reexamine the flavour-violating leptonic decays of the $\mathrm{Z}$ boson in our model, carefully treating the case of a large NHL mass. The diagrams are very similar to the flavour-conserving leptonic decays of the $\mathrm{Z}$ boson discussed in Chapter 6 and we will borrow some results from there; our intention here is to focus on the 
typical features of the flavour-violating processes rather than on the calculational details.

In Sec. 5.2 we continue with the discussion of the sensitivity of the flavourviolating processes in general to the presence of NHL's. We will refer to this discussion later, when in the main part of this work - the calculation of the flavourconserving processes in Chapters 6 and 7 - we will confront our results with those for flavour-violating processes.

\subsection{Flavour-violating leptonic decays of the $\mathrm{Z}$ bo- SOn}

In the SM, the CKM matrix gives rise to the flavour-violating hadronic decays of the $\mathrm{Z}$ boson at the one-loop level. In our model, by analogy, the mixing matrix $K$ (see Sec. 3.2) induces the flavour-violating decays $Z \rightarrow l_{1}^{-} l_{2}^{+}$at the one-loop level $\square$. One-loop Feynman diagrams generating these decays are given in Fig. 5.1. There is no tree-level contribution since there is no mixing between the charged leptons in the neutral current Lagrangian. We will be studying how these graphs contribute to the observable, the width $\Gamma_{l_{1}^{-} l_{2}^{+}}$, in particular the dependence of the width on parameters from the neutrino sector of our model - mixings and NHL masses. The analysis can be simplified (without sacrificing the salient features) by assuming the three NHL's are degenerate, with mass $M_{N}$.

\footnotetext{
${ }^{1}$ This feature is not exclusive to our model. Lepton flavour-violating processes are typical for many other nonstandard models with mixing in the lepton sector.
} 


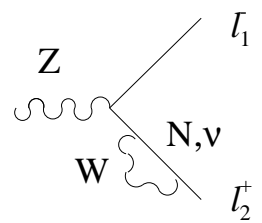

a)

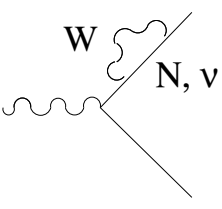

b)

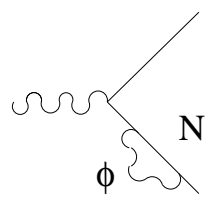

c)

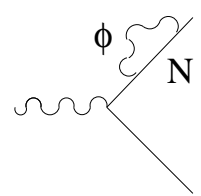

d)

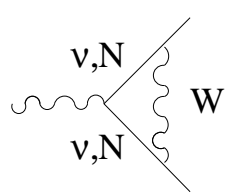

e)

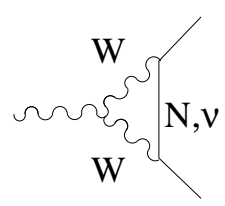

f)

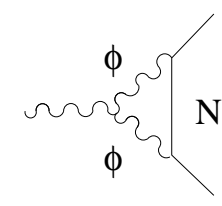

g)

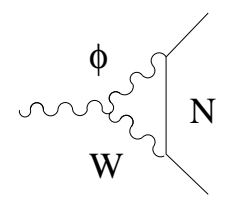

h)

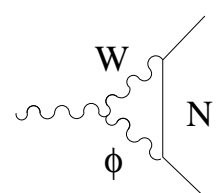

i)

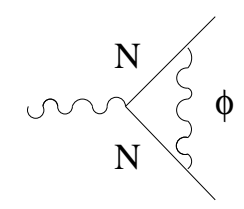

j)

Figure 5.1: One-loop diagrams for flavour-violating leptonic decays of the Z boson.

\subsubsection{The amplitude and the width for $Z \rightarrow l_{1}^{-} l_{2}^{+}$}

The total amplitude $\mathcal{M}$ is given by the sum of partial amplitudes corresponding to the graphs of Fig. 5.1 a-j (subscripts in $\mathcal{M}_{W N} \ldots$ refer to particles in the loop) :

$$
\begin{aligned}
\mathcal{M} & =+i e \epsilon_{\mu} \gamma^{\mu}\left(1-\gamma_{5}\right) \frac{\alpha}{4 \pi}\left\{k_{1} \mathcal{M}_{W N}-k_{1} \mathcal{M}_{W \nu}+k_{1} \mathcal{M}_{\phi N}+k_{4} \mathcal{M}_{\nu \nu W}\right. \\
& +k_{3} \mathcal{M}_{\nu N W}+k_{3} \mathcal{M}_{N \nu W}+k_{2} \mathcal{M}_{N N W}+k_{1} \mathcal{M}_{W W N}-k_{1} \mathcal{M}_{W W \nu} \\
& \left.+k_{1} \mathcal{M}_{\phi \phi N}+k_{1} \mathcal{M}_{\phi W N}+k_{2} \mathcal{M}_{N N \phi}\right\}
\end{aligned}
$$

where $k_{1}, k_{2}, k_{3}, k_{4}$ are mixing factors to be derived shortly and $\epsilon_{\mu}$ is a polarization four-vector of the $Z$ boson. Further, functions $\mathcal{M}_{W N}, \ldots$ depend on masses and momenta of internal and external particles. $\mathcal{M}_{W N}$ is the sum of diagrams 5.1 a, 5.1 $\mathrm{b}$ with $N$ in the loop, $\mathcal{M}_{W \nu}$ is the sum of 5.1 a, 5.1b with $\nu$ in the loop, $\mathcal{M}_{\phi N}$ is the sum

\footnotetext{
${ }^{2}$ We work in the Feynman gauge, see Sec. 4.1
} 
of diagrams 5.1 c, 5.1 d and $\mathcal{M}_{\phi W N}$ is the sum of equal contributions from Fig. 5.1h and 5.1 i. Besides diagrams 5.1 a, 5.1b also 5.1f comes in both with massless $\nu$ 's and NHL's. Diagram 5.1e comes in with four combinations of neutral lepton types. A sample calculation of one function, $\mathcal{M}_{N N \phi}$, will be given in Sec. 6.4.1. Here we simply state results for all functions $\left(\frac{m_{l}^{2}}{M_{W}^{2}}\right.$ terms neglected) :

$$
\begin{aligned}
\mathcal{M}_{W N} & =\frac{-\frac{1}{2}+s_{W}^{2}}{4 s_{W}^{3} c_{W}}\left[\frac{1}{2}-\Delta_{\mu}+\ln M_{W}^{2}+f(\mathcal{X})\right] \\
\mathcal{M}_{W \nu} & =\frac{-\frac{1}{2}+s_{W}^{2}}{4 s_{W}^{3} c_{W}}\left[\frac{1}{2}-\Delta_{\mu}+\ln M_{W}^{2}\right] \\
\mathcal{M}_{\phi N} & =\frac{-\frac{1}{2}+s_{W}^{2}}{4 s_{W}^{3} c_{W}}\left[-\frac{1}{2}-\Delta_{\mu}+\ln M_{W}^{2}+f(\mathcal{X})\right] \frac{\mathcal{X}}{2} \\
\mathcal{M}_{a b W} & =-\frac{1}{8 s_{W}^{3} c_{W}}\left\{2 M _ { Z } ^ { 2 } \left[\left(C_{23}\left(M_{a}, M_{W}, M_{b}\right)+C_{11}\left(M_{a}, M_{W}, M_{b}\right)\right]\right.\right. \\
& \left.+2-4 C_{24}^{f i n}\left(M_{a}, M_{W}, M_{b}\right)-\Delta_{\mu}\right\}, \quad a, b=N, \nu ; M_{\nu}=0 \\
\mathcal{M}_{W W a} & =-\frac{3 c_{W}}{4 s_{W}^{3}}\left\{\frac { 2 } { 3 } M _ { Z } ^ { 2 } \left[-C_{11}\left(M_{W}, M_{a}, M_{W}\right)-C_{23}\left(M_{W}, M_{a}, M_{W}\right)\right.\right. \\
& \left.\left.-C_{0}\left(M_{W}, M_{a}, M_{W}\right)\right]+4 C_{24}^{f i n}\left(M_{W}, M_{a}, M_{W}\right)-\frac{2}{3}+\Delta_{\mu}\right\} \\
\mathcal{M}_{\phi \phi N} & =-\frac{1}{2 s_{W}^{3}} \frac{1-2 s_{W}^{2}}{2 c_{W}} \mathcal{X}\left[C_{24}^{f i n}\left(M_{W}, M_{N}, M_{W}\right)+\frac{1}{4} \Delta_{\mu}\right] \\
\mathcal{M}_{\phi W N} & =+\frac{M_{W}^{2}}{2 s_{W} c_{W}} \mathcal{X} C_{0}\left(M_{W}, M_{N}, M_{W}\right), \\
\mathcal{M}_{N N \phi} & =+\frac{M_{W}^{2}}{8 s_{W}^{3} c_{W}} \mathcal{X}^{2} C_{0}\left(M_{N}, M_{W}, M_{N}\right),
\end{aligned}
$$

where

$$
\begin{aligned}
\mathcal{X} & \equiv \frac{M_{N}^{2}}{M_{W}^{2}}, \quad s_{W} \equiv \sin \theta_{W}, \quad c_{W} \equiv \cos \theta_{W} \\
\Delta_{\mu} & =\frac{2}{\epsilon}-\gamma+\ln 4 \pi+\ln \mu^{2} \\
f(\mathcal{X}) & =\frac{\mathcal{X}^{2} \log \mathcal{X}}{(\mathcal{X}-1)^{2}}+\frac{\mathcal{X}}{1-\mathcal{X}} .
\end{aligned}
$$


Our results are written in terms of the 't Hooft-Veltman integrals $C_{0}, C_{24}, C_{11}$, $C_{23}$ [51] defined in the Appendix E.3. They contain both finite and infinite parts regularized by dimensional regularization [40] (see Appendix D). Infinite parts are parametrized by $\Delta_{\mu}$, where $\epsilon \rightarrow 0$ is not to be confused with the polarization fourvector $\epsilon_{\mu}$.

We now evaluate the mixing parameters. The parameter $k_{1}$ comes from diagrams with one NHL in the loop, $k_{2}$ comes from diagrams with two NHL's, $k_{3}$ from diagrams with one NHL and one massless neutrino and $k_{4}$ from the graph with two massless neutrinos. The case of one massless neutrino is trivial $\left(-k_{1}\right)$ and is not shown below. Starting with terms that come directly from the Feynman rules of Appendix Q, we work our way through to the final form using the properties of the mixing matrix $K$ from Sec. 3.2 (if not explicitly shown, repeated indices are summed over):

$$
\begin{aligned}
k_{1} & =\left(K_{H}\right)_{l a}\left(K_{H}^{\dagger}\right)_{a l^{\prime}}=l l_{m i x}^{\prime}, \\
k_{2} & =\left(K_{H}\right)_{l a}\left(K_{H}^{\dagger} K_{H}\right)_{a b}\left(K_{H}^{\dagger}\right)_{b l^{\prime}}=\sum_{m=e, \mu, \tau}\left(K_{H}\right)_{l a}\left(K_{H}^{\dagger}\right)_{a m}\left(K_{H}\right)_{m b}\left(K_{H}^{\dagger}\right)_{b l^{\prime}} \\
& =\sum_{m=e, \mu, \tau} l m_{m i x} m l_{m i x}^{\prime}, \\
k_{3} & =\left(K_{L}\right)_{l i}\left(K_{L}^{\dagger} K_{H}\right)_{i a}\left(K_{H}^{\dagger}\right)_{a l^{\prime}}=\sum_{m=e, \mu, \tau}\left(K_{L}\right)_{l i}\left(K_{L}^{\dagger}\right)_{i m}\left(K_{H}\right)_{m a}\left(K_{H}^{\dagger}\right)_{a l^{\prime}} \\
& =\sum_{m}\left[\delta_{l m}-\left(K_{H}\right)_{l b}\left(K_{H}^{\dagger}\right)_{b m}\right]\left(K_{H}\right)_{m a}\left(K_{H}^{\dagger}\right)_{a l^{\prime}}=l l_{m i x}^{\prime}-\sum_{m} l m_{m i x} m l_{m i x}^{\prime} \\
& =k_{1}-k_{2}, \\
k_{4} & =\left(K_{L}\right)_{l i}\left(K_{L}^{\dagger} K_{L}\right)_{i j}\left(K_{L}^{\dagger}\right)_{j l^{\prime}}=-2 k_{1}+k_{2} .
\end{aligned}
$$

For $k_{4}$ we show only the initial and final step.

To address the question of infinities, we note that we do not have to actually renormalize. Indeed, we easily observe the mass independent divergences (in fact any terms independent of mass) are cancelled in the sums

$$
\mathcal{M}_{W W \nu}+\mathcal{M}_{W W N}
$$




$$
\begin{array}{r}
\mathcal{M}_{N \nu W}+\mathcal{M}_{\nu N W}+\mathcal{M}_{N N W}+\mathcal{M}_{\nu \nu W}, \\
\mathcal{M}_{W \nu}+\mathcal{M}_{W N} .
\end{array}
$$

The origin of this, so-called GIM cancellation can be traced back to the unitarity of the mixing matrix $K{ }^{3}$. The remaining divergent amplitudes have their divergence multiplied by the mass term $\mathcal{X}$ and therefore GIM cancellation does not apply here. However, these divergences vanish in the sum of mass dependent diagrams,

$$
\mathcal{M}_{\phi \phi N}+\mathcal{M}_{\phi N}
$$

Using Eq. 5.4 it can be shown that the width for the flavour-violating decays of the $\mathrm{Z}$ boson to $l_{1}^{-} l_{2}^{+}$is given in terms of $k_{1}$ and $k_{2}$ as

$$
\Gamma_{l_{1}^{-} l_{2}^{+}}=\frac{2}{3} \frac{\alpha^{3}}{(4 \pi)^{2}} M_{Z}\left|k_{1} \mathcal{M}_{1}+k_{2} \mathcal{M}_{2}\right|^{2}
$$

where

$$
\begin{aligned}
\mathcal{M}_{1} & =\mathcal{M}_{\phi W N}+\mathcal{M}_{\phi \phi N}-\mathcal{M}_{W W \nu}+\mathcal{M}_{W W N}+\mathcal{M}_{N \nu W}+\mathcal{M}_{\nu N W}-2 \mathcal{M}_{\nu \nu W} \\
& -\mathcal{M}_{W \nu}+\mathcal{M}_{\phi N}+\mathcal{M}_{W N} \\
\mathcal{M}_{2} & =\mathcal{M}_{N N \phi}-\mathcal{M}_{N \nu W}-\mathcal{M}_{\nu N W}+\mathcal{M}_{\nu \nu W}+\mathcal{M}_{N N W} .
\end{aligned}
$$

The amplitude squared can be written as

$$
\left|k_{1} \mathcal{M}_{1}+k_{2} \mathcal{M}_{2}\right|^{2}=\left|k_{1}\right|^{2}\left|\mathcal{M}_{1}\right|^{2}+\left|k_{2}\right|^{2}\left|\mathcal{M}_{2}\right|^{2}+2 R e\left(k_{1} k_{2}^{*} \mathcal{M}_{1} \mathcal{M}_{2}^{*}\right)
$$

The mixing factors $k_{1}, k_{2}$ are process dependent and the following relations hold

\footnotetext{
${ }^{3}$ This cancellation is referred to as the GIM cancellation since it has a similar origin as the cancellations due to the CKM matrix in $K^{0} \rightarrow \mu^{+} \mu^{-}$which lead to the postulation of the $c$ quark by Glashow, Iliopoulos and Maiani (GIM) [6].
} 
between $\mathrm{CP}$ conjugate final states:

$$
k_{1,2} \equiv k_{1,2}\left(l_{1}^{-} l_{2}^{+}\right)=k_{1,2}^{*}\left(l_{1}^{+} l_{2}^{-}\right)
$$

implying f

$$
\begin{aligned}
\operatorname{Re}\left\{k_{1}\left(l_{1}^{-} l_{2}^{+}\right) k_{2}^{*}\left(l_{1}^{-} l_{2}^{+}\right) \mathcal{M}_{1} \mathcal{M}_{2}^{*}\right\} & +\operatorname{Re}\left\{k_{1}\left(l_{1}^{+} l_{2}^{-}\right) k_{2}^{*}\left(l_{1}^{+} l_{2}^{-}\right) \mathcal{M}_{1} \mathcal{M}_{2}^{*}\right\} \\
& =2 \operatorname{Re}\left(k_{1} k_{2}^{*}\right) \operatorname{Re}\left(\mathcal{M}_{1} \mathcal{M}_{2}^{*}\right)
\end{aligned}
$$

giving the total rate for $Z \rightarrow l_{1}^{+} l_{2}^{-}+l_{1}^{-} l_{2}^{+}$

$$
\begin{aligned}
\Gamma_{l_{1}^{-} l_{2}^{+}+l_{1}^{+} l_{2}^{-}} & =\frac{4}{3} \frac{\alpha^{3}}{(4 \pi)^{2}} M_{Z}\left\{\left|k_{1}\right|^{2}\left|\mathcal{M}_{1}\right|^{2}+\left|k_{2}\right|^{2}\left|\mathcal{M}_{2}\right|^{2}+2 \operatorname{Re}\left(k_{1} k_{2}^{*}\right)\right. \\
& \left.\times \operatorname{Re}\left(\mathcal{M}_{1} \mathcal{M}_{2}^{*}\right)\right\}
\end{aligned}
$$

\subsubsection{Approximate relations in the limit of large NHL mass}

While we can easily see how the width $\Gamma_{l_{1}^{-}} l_{2}^{+}+l_{1}^{+} l_{2}^{-}$depends on mixing factors, the dependence on the NHL mass $M_{N}$ is obscured by the algebraic complexity of the 't Hooft - Veltman integrals. Fortunately, in the most interesting case, which is that of a large $M_{N}$, the amplitudes become particularly simple. It is the most interesting case since the signal is the largest due to quadratic nondecoupling effects. This means

\footnotetext{
${ }^{4}$ Note the difference

$$
\begin{aligned}
\operatorname{Re}\left\{k_{1}\left(l_{1}^{-} l_{2}^{+}\right) k_{2}^{*}\left(l_{1}^{-} l_{2}^{+}\right) \mathcal{M}_{1} \mathcal{M}_{2}^{*}\right\} & -\operatorname{Re}\left\{k_{1}\left(l_{1}^{+} l_{2}^{-}\right) k_{2}^{*}\left(l_{1}^{+} l_{2}^{-}\right) \mathcal{M}_{1} \mathcal{M}_{2}^{*}\right\} \\
& =-2 \operatorname{Im}\left(k_{1} k_{2}^{*}\right) \operatorname{Im}\left(\mathcal{M}_{1} \mathcal{M}_{2}^{*}\right)
\end{aligned}
$$
}

may lead to a $\mathrm{CP}$ violating asymmetry

$$
\eta \equiv \frac{\Gamma_{l_{1}^{-} l_{2}^{+}}-\Gamma_{l_{1}^{+} l_{2}^{-}}}{\Gamma_{Z}}=-\frac{8}{3} \frac{\alpha^{3}}{(4 \pi)^{2}} \frac{M_{Z}}{\Gamma_{Z}} \operatorname{Im}\left(k_{1} k_{2}^{*}\right) \operatorname{Im}\left(\mathcal{M}_{1} \mathcal{M}_{2}^{*}\right) .
$$

We found that the maximum value allowed, $\eta \leq 2.2 \times 10^{-14}$ for $e \tau$ mode at $M_{N}=5 \mathrm{TeV}$, is very small (see experimental limits in Eq. 5.22). 
that some of the diagrams give rise to terms $O\left(M_{N}^{2}\right)$. These effects, as well as the question of how high we can go with the mass $M_{N}$ without disturbing perturbation theory, will be discussed in Secs. 6.6 and 6.7. For now, we just state that by large NHL mass we mean $M_{Z}<M_{N}<5 \mathrm{TeV}$.

In the limit of a large NHL mass $M_{N}$, the amplitudes exhibit the following behaviour:

$$
\begin{gathered}
\mathcal{M}_{W W N}=-\frac{3 c_{W}}{4 s_{W}^{3}}\left\{4 C_{24}^{f i n}\left(M_{W}, M_{N}, M_{W}\right)-\frac{2}{3}+\Delta_{\mu}\right\} \\
\mathcal{M}_{a b W}=-\frac{1}{8 s_{W}^{3} c_{W}}\left\{2-4 C_{24}^{f i n}\left(M_{a}, M_{W}, M_{b}\right)-\Delta_{\mu}\right\} \\
a, b=N, \nu ; \nu, N ; N, N ; \quad M_{\nu}=0 .
\end{gathered}
$$

These formulae differ by less than one percent from the exact ones in Eq. 5.2, at $M_{N}=500 \mathrm{GeV}$ and the difference decreases with rising $M_{N}$ to less than 0.1 percent at $M_{N}=5000 \mathrm{GeV}$. $C$ functions in the same limit behave as

$$
\begin{aligned}
C_{0}\left(M_{W}, M_{N}, M_{W}\right) & =\frac{1}{M_{N}^{2}}\left[\ln \mathcal{X}+2 \sqrt{4 c_{W}^{2}-1}\left(\theta-\frac{\pi}{2}\right)+1\right. \\
& \left.+O\left(\mathcal{X}^{-1}\right)\right], \quad \theta=\arctan \sqrt{4 c_{W}^{2}-1}, \\
C_{0}\left(M_{N}, M_{W}, M_{N}\right) & =\frac{1}{M_{N}^{2}}\left[1+O\left(\mathcal{X}^{-1}\right)\right], \\
C_{24}^{f i n}\left(M_{W}, M_{N}, M_{W}\right) & =\frac{3}{8}-\frac{1}{4} \ln M_{N}^{2}+O\left(\mathcal{X}^{-1}\right),
\end{aligned}
$$

and also $C_{24}^{\text {fin }}$ function of any other combination of arguments involving $M_{N}$ varies slowly as $\ln M_{N}^{2}$.

With the help of Eqs. 5.13, 5.14, we can see there are three amplitudes in Eq. 5.2 with nondecoupling behaviour, namely the quadratic dependence on NHL mass. They are $\mathcal{M}_{N N \phi}, \mathcal{M}_{\phi \phi N}$ and $\mathcal{M}_{\phi N}$. However, as numerical calculations show, $\mathcal{M}_{\phi \phi N} \rightarrow-\mathcal{M}_{\phi N}$ for large $M_{N}$, leaving us $\mathcal{M}_{N N \phi}$ as the only amplitude with the nondecoupling behaviour. $\mathcal{M}_{N N \phi}$ gives the dominant contribution to $\mathcal{M}_{2}$ and, more- 
over, it ensures that for large $M_{N}$

$$
\left|k_{2}\right|^{2}\left|\mathcal{M}_{2}\right|^{2}>\left|k_{1}\right|^{2}\left|\mathcal{M}_{1}\right|^{2}
$$

despite the fact that the $\left|k_{2}\right|$ is quadratically small compared to the linear $\left|k_{1}\right|$. In Refs. [26, 46] the authors neglected terms proportional to $\left|k_{2}\right|$, therefore their results do not apply in the large $M_{N}$ limit.

\subsubsection{Numerical results}

In the numerical calculations, the term $R e\left(k_{1} k_{2}^{*}\right)$ from the Eq. 5.12 was treated as follows. We only have limits on $\left|k_{1}\right|,\left|k_{2}\right|$ (input parameters for our calculations), not on real and imaginary parts of $k_{1}, k_{2}$. Thus for given $\left|k_{1}\right|,\left|k_{2}\right|$, the real part of $k_{1} k_{2}^{*}$ can vary as

$$
-\left|k_{1}\right|\left|k_{2}\right| \leq \operatorname{Re}\left(k_{1} k_{2}^{*}\right) \leq\left|k_{1}\right|\left|k_{2}\right|
$$

In our calculations we set

$$
R e\left(k_{1} k_{2}^{*}\right)=\delta\left|k_{1}\right|\left|k_{2}\right|
$$

and $\delta$ is varied between -1 and +1 as an independent input parameter. To find a numerical value of the parameter $\left|k_{2}\right|^{2}$, we express it in terms of $l l_{\text {mix }}$ and $l_{1} l_{2 \text { mix }}$,

$$
\begin{aligned}
\left|k_{2}\right|^{2} & \equiv\left|k_{2}\right|^{2}\left(l_{1}^{-} l_{2}^{+}\right)=\left(l_{1} l_{1 \text { mix }}+l_{2} l_{2 m i x}\right)^{2}\left|l_{1} l_{2 m i x}\right|^{2}+\left|l_{1} l_{3 m i x}\right|^{2}\left|l_{3} l_{2 m i x}\right|^{2} \\
& +2\left(l_{1} l_{1 \text { mix }}+l_{2} l_{2 \text { mix }}\right) \operatorname{Re}\left\{l_{1} l_{2 \text { mix }}^{*} l_{1} l_{3 \text { mix }} l_{3} l_{2 m i x}\right\} .
\end{aligned}
$$


The smallness of $e \mu_{m i x}$ effectively removes some of the terms. For the $e \mu$ final state, the first and the third terms above are negligible, leaving

$$
\left|k_{2}\right|^{2}(e \mu) \doteq\left|e \tau_{m i x}\right|^{2}\left|\tau \mu_{m i x}\right|^{2}
$$

while for $e \tau$ and $\mu \tau$ sector we have

$$
\begin{aligned}
\left|k_{2}\right|^{2}(e \tau) & \doteq\left(e e_{\text {mix }}+\tau \tau_{\text {mix }}\right)^{2}\left|e \tau_{\text {mix }}\right|^{2} \\
\left|k_{2}\right|^{2}(\mu \tau) & \doteq\left(\mu \mu_{\text {mix }}+\tau \tau_{\text {mix }}\right)^{2}\left|\mu \tau_{\text {mix }}\right|^{2}
\end{aligned}
$$

The maximally allowed mixings (Eqs. 3.38, 3.45, 3.44) imply $\left|k_{1}\right|=(0.00024$, $0.015,0.0068)$ and $\left|k_{2}\right|=(0.0001,0.0006,0.00023)$ for $e \mu, e \tau, \mu \tau$ modes respectively. As noted before, we assume degenerate NHL's with mass $M_{N}$. Gauge boson masses used in the numerical calculations are $M_{Z}=91.1884 \mathrm{GeV}$ [52 and $M_{W}=80.410 \mathrm{GeV}$ [53]. The total decay width of the $\mathrm{Z}$ boson is taken as $\Gamma_{Z}=2.4963 \mathrm{GeV}$ [52].

The results are shown in Fig. 5.2a,b. They show how the branching ratio $B R\left(l_{1}^{ \pm} l_{2}^{\mp}\right) \equiv \Gamma_{l_{1}^{+} l_{2}^{-}+l_{1}^{-} l_{2}^{+}} / \Gamma_{Z}$ varies with the NHL mass. In Fig. 5.2a we set $\delta=-1$, in Fig. 5.2 b $\delta=+1$. The graphs start at $M_{N}=100 \mathrm{GeV}$. For NHL masses less than $M_{W}$, the rates are negligibly small. A sudden rise in branching ratio just above $M_{N}=1 \mathrm{TeV}$ for $e \tau$ and $\mu \tau$ modes in Fig. 5.2a signals that at this point the $\left|k_{2}\right|^{2}\left|\mathcal{M}_{2}\right|^{2}$ term overtakes the $\left|k_{1}\right|^{2}\left|\mathcal{M}_{1}\right|^{2}$ term and the nondecoupling behaviour (generated by the Feynman graph Fig. 5.1j) becomes dominant. We predict the following branching ratio limits for $M_{N}=5 \mathrm{TeV}$ and $\delta=+1$ :

$$
\begin{aligned}
& B R_{t h}\left(Z \rightarrow e^{ \pm} \mu^{\mp}\right)<3.3 \times 10^{-8}, \\
& B R_{t h}\left(Z \rightarrow e^{ \pm} \tau^{\mp}\right)<1.4 \times 10^{-6}, \\
& B R_{t h}\left(Z \rightarrow \mu^{ \pm} \tau^{\mp}\right)<2.2 \times 10^{-7} .
\end{aligned}
$$


These results are similar to those of Ref. 448, where the calculation was done in the context of a see-saw model with enhanced mixings.

Current experimental upper limits on branching ratios are [2]

$$
\begin{aligned}
B R_{\text {exp }}\left(Z \rightarrow e^{ \pm} \mu^{\mp}\right) & <6 \times 10^{-6}, \quad 95 \% \text { C.L. } \\
B R_{\text {exp }}\left(Z \rightarrow e^{ \pm} \tau^{\mp}\right) & <1.3 \times 10^{-5}, \quad 95 \% \text { C.L. } \\
B R_{\text {exp }}\left(Z \rightarrow \mu^{ \pm} \tau^{\mp}\right) & <1.9 \times 10^{-5}, \quad 95 \% \text { C.L. }
\end{aligned}
$$

Our prediction for $e \tau$ mode is thus at least one order of magnitude below the experimental limit.

\subsection{Flavour-violating processes at very low ener- gies}

How does this compare with flavour-violating processes at very low energies ? The rare decay $\mu \rightarrow e \gamma$ (see Fig. 3.1) is very well measured and supplies us with a stringent limit on $\left|e \mu_{m i x}\right|$ (see Eq. 3.44), which we use as an input parameter for our calculations. We now derive this limit. The decay $\mu \rightarrow e \gamma$ was studied in the context of our model and see-saw models with enhanced mixings by several authors [20, 42, 43, 44, 45]. In our model, with mass degenerate NHL's, the $\mu \rightarrow e \gamma$ branching ratio is 43

$$
B R(\mu \rightarrow e \gamma)=\frac{3 \alpha}{32 \pi}\left|e \mu_{m i x}\right|^{2}\left|F_{\gamma}(\mathcal{X})\right|^{2}
$$



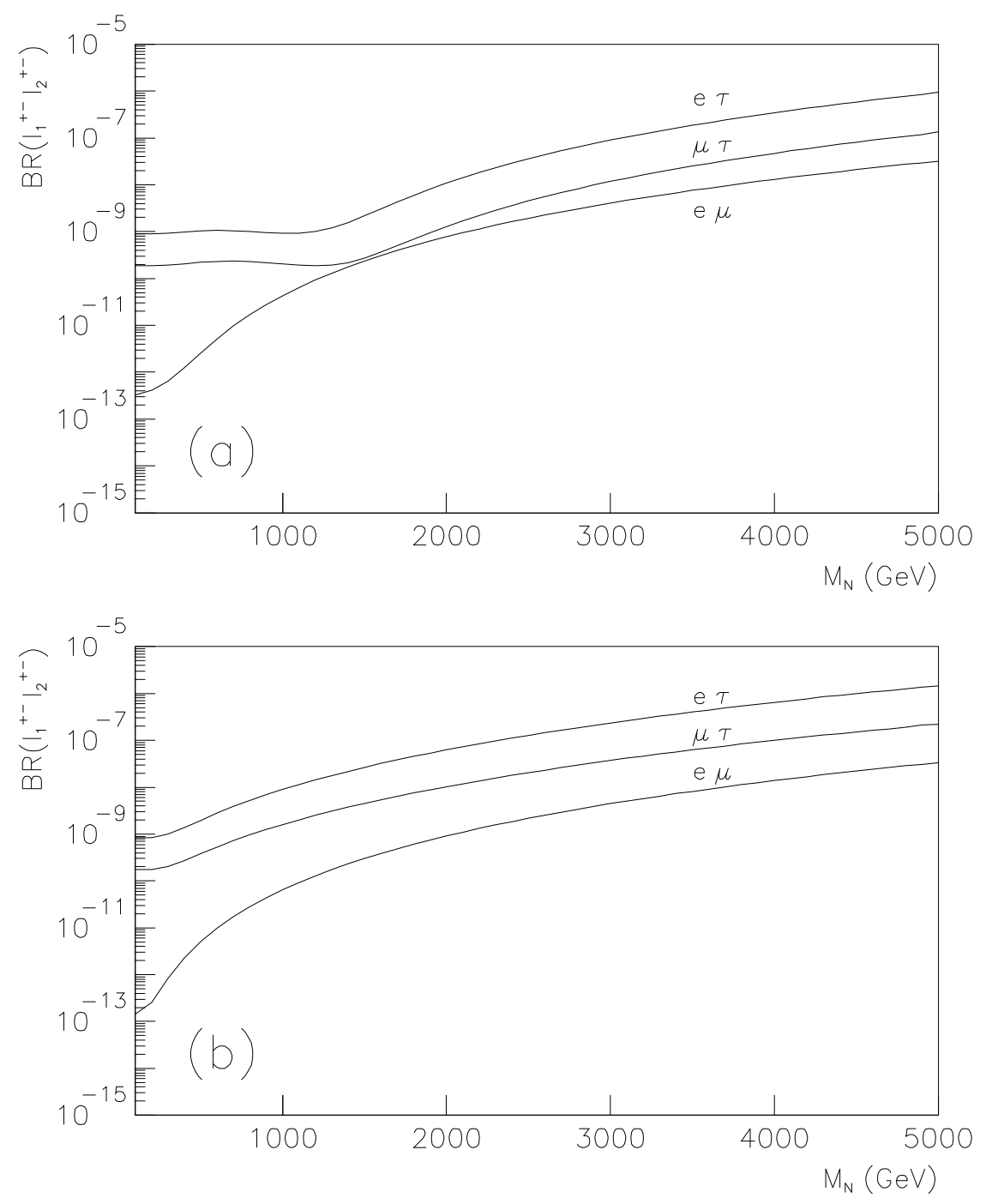

Figure 5.2: The branching ratio $Z \rightarrow l_{1}^{ \pm} l_{2}^{\mp}$ as a function of $M_{N}$ for (a) $\delta=-1$, (b) $\delta=+1$. 
where

$$
\left|F_{\gamma}(\mathcal{X})\right|^{2}=-\mathcal{X} \frac{1-5 \mathcal{X}-2 \mathcal{X}^{2}}{(1-\mathcal{X})^{3}}+\frac{6 \mathcal{X}^{3}}{(1-\mathcal{X})^{4}} \ln \mathcal{X}, \quad \mathcal{X}=\frac{M_{N}^{2}}{M_{W}^{2}}
$$

is an NHL mass dependent form factor. For NHL masses $M_{N}>500 \mathrm{GeV}$, which we ultimately consider, the formfactor becomes independent of mass,

$$
F_{\gamma}(\mathcal{X}) \rightarrow-2
$$

This is another example of the nondecoupling behaviour $\left(F_{\gamma}(\mathcal{X})\right.$ does not vanish $)$ of NHL's 5 . It is the mildest nondecoupling case; we encountered in the previous section amplitudes with quadratic dependence on NHL mass. Given the current experimental limit on the $\mu \rightarrow e \gamma$ branching ratio [2],

$$
B R(\mu \rightarrow e \gamma) \leq 4.9 \times 10^{-11} \quad 90 \% \text { C.L. }
$$

Eqs. 5.23, 5.25 yield an upper limit on the mixing of $\left|e \mu_{m i x}\right| \leq 0.00024$, given previously as Eq. 3.44.

\footnotetext{
${ }^{5}$ It is instructive to see how this result arises from the dimensional analysis argument. The effective Lagrangian for the $\mu \rightarrow e \gamma$ is given by 54$$
\mathcal{L}_{e f f}=T(\mathcal{X}) \bar{e}_{L} \sigma_{\lambda \nu} \mu_{R} F^{\lambda \nu}
$$

where the field operators $\bar{e}_{L}, \mu_{R}, F^{\lambda \nu}$ have mass dimensions $3 / 2,3 / 2$ and 2 , respectively; hence $T(\mathcal{X})$ has to have dimension -1 . For Fig. 3.1, the large mass $M_{N}$ dominance thus suggests $T(\mathcal{X}) \sim \frac{1}{M_{N}}$ on dimensional grounds. However, there is also a possibility of $T(\mathcal{X}) \sim \frac{m_{\mu}}{M_{N}^{2}} ;$ this is indeed what happens since it is $m_{\mu}\left(\right.$ or $m_{e}$ ) which gives the right helicity flip to yield the required $\mathcal{L}_{\text {eff }}$ (these points can be best understood after writing down the amplitude for the graph). The amplitude for Fig. 3.1 thus decouples quadratically.

There is another graph where two internal W's are replaced by the unphysical Higgs $\phi$. The large $M_{N}$ behaviour is in this case boosted by $M_{N}$ dependent couplings of NHL's to $\phi$ 's, so this dominant graph yields $T(\mathcal{X}) \sim F_{\gamma}(\mathcal{X}) \sim \frac{m_{\mu}}{M_{N}^{2}} \frac{M_{N}^{2}}{M_{W}^{2}} \sim$ const, in agreement with Eq. 5.25.
} 
On the other hand, experimental limits on $\tau \rightarrow e \gamma$ and $\tau \rightarrow \mu \gamma$ [2],

$$
\begin{aligned}
& B R_{\exp }(\tau \rightarrow e \gamma)<1.2 \times 10^{-4}, \quad 90 \% \text { C.L. } \\
& B R_{\text {exp }}(\tau \rightarrow \mu \gamma)<4.2 \times 10^{-6}, \quad 90 \% \text { C.L. },
\end{aligned}
$$

are much weaker. The predicted rate for both $e \gamma$ and $\mu \gamma$ modes is [43]

$$
B R_{t h}(\tau \rightarrow e \gamma, \mu \gamma)=7 \times 10^{-7}, \quad \text { for } \quad M_{N}>500 \mathrm{GeV}
$$

However, the limits on mixing parameters used in Ref. [13] are out of date now. With the current limits the predicted rate would be smaller by at least one order of magnitude, implying that the theoretical result is two orders of magnitude below the experimental upper limit for $\mu \gamma$ mode and about three orders for $e \gamma$ mode. This explains why we had to use indirect limits of Eq. 3.45 for $\mu \tau_{m i x}$ and $e \tau_{m i x}$.

Another well-measured muon decay mode is $\mu \rightarrow e^{-} e^{-} e^{+}$, with [2]

$$
B R_{\exp }\left(\mu \rightarrow e^{-} e^{-} e^{+}\right)<1.0 \times 10^{-12}, \quad 90 \% \text { C.L. } .
$$

This process was considered by Refs. [20, 42, 44, 45]. The calculation shows the quadratic nondecoupling we encountered in the lepton flavour-violating decays of the $\mathrm{Z}$ boson. Ref. 45 gives (with an assumption discussed therein) the following constraint for the parameters of the superstring-inspired (our) model:

$$
e e_{m i x}\left|e \mu_{m i x}\right| \leq 0.93 \times 10^{-5} \frac{1 \mathrm{TeV}^{2}}{M_{N}^{2}\left(\mathrm{TeV}^{2}\right)},
$$

which for $M_{N} \geq 3 \mathrm{TeV}$ is competitive with a constraint implied by Eqs. 3.38, 3.45, 3.44:

$$
e e_{m i x}\left|e \mu_{m i x}\right| \leq 0.17 \times 10^{-5} .
$$


Also considered in Refs. [20, 42, 45] is $\mu-e$ conversion in nuclei, $\mu^{-}(A, Z) \rightarrow$ $e^{-}(A, Z)$. The constraint on the product $e e_{\text {mix }}\left|e \mu_{m i x}\right|$ [45] is similar to the one above.

For the flavour-violating decays of the tau into three leptons $\left(\tau \rightarrow e^{-} e^{-} e^{+}\right.$, $e^{-} \mu^{-} \mu^{+}$, etc) there is to my knowledge no calculation studying the large (TeV) NHL mass limit in the context of our model. Within the see-saw model of Ref. [19], Pilaftsis predicts with the current limits on mixings and for $M_{N}=3 \mathrm{TeV}$ [48]:

$$
\begin{aligned}
B R_{t h}\left(\tau \rightarrow e^{-} e^{-} e^{+}\right) & =5 \times 10^{-7}, \\
B R_{t h}\left(\tau \rightarrow e^{-} \mu^{-} \mu^{+}\right) & =3 \times 10^{-7} .
\end{aligned}
$$

The current experimental limits are [2]

$$
\begin{aligned}
& B R_{e x p}\left(\tau \rightarrow e^{-} e^{-} e^{+}\right)<1.4 \times 10^{-5}, \quad 90 \% \text { C.L. }, \\
& B R_{\text {exp }}\left(\tau \rightarrow e^{-} \mu^{-} \mu^{+}\right)<1.4 \times 10^{-5}, \quad 90 \% \text { C.L. . }
\end{aligned}
$$

Finally, hadronic decay modes of the $\tau$ lepton, $\tau \rightarrow l \eta, l \pi^{0}$ [13] are disfavoured by loose limits, e.g. $B R\left(\tau \rightarrow \mu^{-} \pi^{0}\right)<4.4 \times 10^{-5}$ [2].

In conclusion, to probe large NHL masses, we would have to push experimental upper limits by at least one order of magnitude for flavour-violating leptonic decays of the $\mathrm{Z}$ boson, and by one to two orders of magnitude for flavour-violating decays of the $\tau$ lepton. This most likely requires increased high luminosity running at LEP I energy and $\tau$ factory [55]. Note the dominant contribution to the total rate for $Z \rightarrow l_{1}^{+} l_{2}^{-}+l_{1}^{-} l_{2}^{+},\left|k_{2}\right|^{2}\left|\mathcal{M}_{2}\right|^{2}$, depends quartically on small mixings (see Eqs. 5.19, 5.20) and also quartically on the NHL mass $M_{N}$. Further mass independent limits on mixings will therefore suppress this dominant contribution rather quickly, unless $M_{N}$ is very large. 


\section{Chapter 6}

\section{Lepton flavour-conserving}

\section{processes}

In this and the following chapter we will examine two lepton flavour-conserving processes: i) $Z \rightarrow l^{+} l^{-}(l=e, \mu, \tau)$ with observables $\Gamma_{l l}$ (the width) and $U_{b r}$ (universality breaking parameter); and ii) $\mu \rightarrow e \nu \nu$ with observable $M_{W}$ (the $\mathrm{W}$ boson mass). We will show that these observables probe the mixings vs NHL mass parameter space of our model in many respects more efficiently than the flavour-violating decays discussed in the previous chapter. We work to the one-loop $(O(\alpha))$ level of perturbation theory.

In Sec. 6.1 we classify, closely following the SM case of Ref. [34, one-loop corrections to $Z \rightarrow l^{+} l^{-}$into three groups - oblique, vertex and QED corrections. Each group is then individually studied in Secs. 6.2- 6.4. We note that a large number of contributing diagrams comes directly from the SM without being modified by NHL's. In such cases we use the SM results of Ref. [34]. As far as non-SM contributions are concerned, we present a detailed calculation of two Feynman diagrams (one oblique and one vertex) and a summary of results for the remaining ones. Divergent results are then renormalized as discussed in Chapter 4. 
Secs. $6.5-6.7$ are less technical and hopefully more intriguing. We show the impact of loop corrections to a $\mu$-decay on the $\mathrm{Z}$ width, calculate the $\mathrm{W}$ mass $M_{W}$, discuss the violation of the decoupling theorem and the quadratic dependence of the loop corrections on the NHL mass, and the limitations of the perturbative calculations.

Our numerical results are presented in Sec. 6.8 and discussed in Sec. 6.9. We investigate here only a part of the mixings vs NHL mass parameter space by setting $e e_{m i x}=\mu \mu_{m i x}=0$. The full space is studied in Chapter 7 .

\section{1 $\mathrm{Z} \rightarrow \mathrm{l}^{+} \mathrm{l}^{-}$: the tree-level and the corrections}

The tree-level leptonic width of the $\mathrm{Z}$ boson in the SM is given by

$$
\Gamma_{0}=\frac{\alpha}{3} M_{Z}\left(v_{l}^{2}+a_{l}^{2}\right)
$$

with $v_{l}=\left(-1+4 s_{W}^{2}\right) /\left(4 s_{W} c_{W}\right)$ and $a_{l}=-1 /\left(4 s_{W} c_{W}\right)$ being, respectively, the vector and axial vector couplings of the charged leptons to $Z$. We neglected terms proportional to $m_{l}^{2} / M_{W}^{2}$. In this approximation, as a consequence of the lepton universality of the SM, the partial widths for all three modes $(e e, \mu \mu, \tau \tau)$ are equal.

One-loop corrected leptonic decays of the Z boson in the SM were thoroughly discussed by W. Hollik in Ref. [34]. He parametrizes the leptonic width as

$$
\Gamma_{l l}=\frac{\Gamma_{0}+\delta \hat{\Gamma}_{l l}}{1+\hat{\Pi}_{Z}\left(M_{Z}^{2}\right)}\left(1+\delta_{Q E D}\right) .
$$

The one-loop electroweak corrections include $\mathrm{Z}$ boson propagator (so-called oblique) corrections $\hat{\Pi}_{Z}$; vertex corrections $\delta \hat{\Gamma}_{l l}$ and QED corrections $\delta_{Q E D}$. To give the reader some feeling for the numbers involved, we note that the SM prediction with 
$M_{Z}=91.1884 \mathrm{GeV}, m_{t}=176 \mathrm{GeV}$ and $M_{H}=200 \mathrm{GeV}$ is

$$
\begin{aligned}
& \Gamma_{0}=81.45 \mathrm{MeV}, \\
& \Gamma_{l l}=84.03 \mathrm{MeV},
\end{aligned}
$$

i.e., loops account for $\Gamma_{l l}-\Gamma_{0} \doteq 2.5 \mathrm{MeV}$. The current experimental value under the assumption of lepton universality is [52]

$$
\Gamma_{l l}^{e x p}=83.93 \pm 0.14 \mathrm{MeV}
$$

Without assuming universality [52],

$$
\begin{aligned}
& \Gamma_{e e}^{e x p}=83.92 \pm 0.17 \mathrm{MeV}, \\
& \Gamma_{\mu \mu}^{e x p}=83.92 \pm 0.23 \mathrm{MeV}, \\
& \Gamma_{\tau \tau}^{e x p}=83.85 \pm 0.29 \mathrm{MeV} .
\end{aligned}
$$

In our model, Eqs. 6.1, 6.2 keep the same form. It is $\hat{\Pi}_{Z}$ and $\delta \hat{\Gamma}_{l l}$ which are modified by the contribution of NHL's. Also, $\Gamma_{0}$ is modified (via $s_{W}$ ) in an indirect way (see Sec. 6.5); the QED parameter $\delta_{Q E D}$ is not affected by NHL's. We now address these corrections one by one, starting with $\delta_{Q E D}$.

\subsection{QED corrections}

QED corrections (Fig. 6.1) form a gauge invariant subset and therefore can be treated independently of the genuine electroweak corrections 34. The graphs of Fig. 6.1 were calculated in Ref. [56] where the results were shown to modify the 


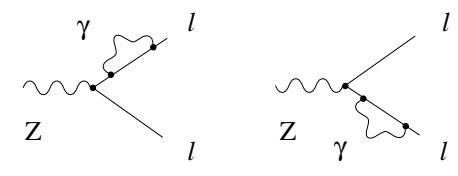

a)

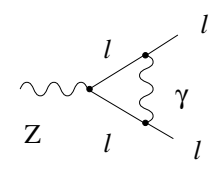

c)

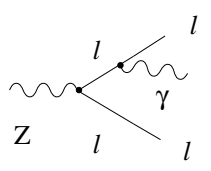

d)

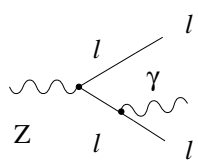

e)

Figure 6.1: QED corrections.

$\mathrm{Z}$ width by a factor $\delta_{Q E D}$ (see Eq. 6.2),

$$
\delta_{Q E D}=\frac{3 \alpha}{4 \pi} .
$$

Our inclusion of NHL's has no impact on this SM result.

\subsection{Z-propagator corrections $\hat{\Pi}_{Z}$}

Z-propagator corrections $\hat{\Pi}_{Z}$ are related to the real part of the renormalized Z selfenergy $\hat{\Sigma}_{Z}$ via

$$
\hat{\Pi}_{Z}\left(M_{Z}^{2}\right)=\frac{\partial \mathcal{R} e \hat{\Sigma}_{Z}}{\partial p^{2}}\left(M_{Z}^{2}\right),
$$

where $p$ is the 4-momentum of the $\mathrm{Z}$ boson. $\hat{\Sigma}_{Z}$ includes, besides the unrenormalized Z self-energy $\Sigma_{Z}$, through the renormalization constant $\delta Z_{2}^{Z}$ (see Eq. E.4), also all other unrenormalized gauge boson self-energies $\Sigma_{W}, \Sigma_{\gamma}$ and $\Sigma_{\gamma Z}$. The diagrams contributing to these self-energies are in Figs. 6.2-6.5.

The photon self-energy (Fig. 6.2) and the photon - Z mixing energy (Fig. 6.3) are not modified by the NHL's (the sum of the fermion loops runs over all fermions except 
neutrinos) and therefore we will use the SM analytical formulae of Refs. [34, 57] given in Eqs. E.5, E.6.

The Z self-energy (Fig. 6.4) and the W self-energy (Fig. 6.5), are modified in our model as NHL's enter the fermion loops. The non-SM graphs from Figs. 6.4, 6.5 are shown explicitly in Fig. 6.6. They include the graphs with massless neutrinos (no NHL's), since these differ from the SM in the mixing factors. We will calculate these graphs and the resulting amplitudes will replace the SM neutrino contribution in Eq. E.7.

$\Sigma_{Z}$ is associated with the transverse $\left(g^{\mu \nu}\right)$ part of the Z self-energy tensor $\Sigma_{Z}^{\mu \nu}$ :

$$
\Sigma_{Z}^{\mu \nu}=g^{\mu \nu} \Sigma_{Z}+p^{\mu} p^{\nu} \tilde{\Sigma}_{Z}
$$

The longitudinal part $\tilde{\Sigma}_{Z}$ does not contribute to S-matrix elements [58 and we will not consider it here. The non-SM part of the Z (unrenormalized) self-energy tensor $\Sigma_{Z}^{\mu \nu}$ is the sum of four terms corresponding to Figs. 6.6 $a-d$ :

$$
\Sigma_{Z}^{\mu \nu}=\Sigma_{Z}^{\mu \nu}\left(M_{N}, M_{N}\right)+\Sigma_{Z}^{\mu \nu}\left(M_{N}, 0\right)+\Sigma_{Z}^{\mu \nu}\left(0, M_{N}\right)+\Sigma_{Z}^{\mu \nu}(0,0) .
$$

We evaluate the contribution of one of these terms, $\Sigma_{Z}^{\mu \nu}\left(M_{N}, M_{N}\right)$ in detail below as an example. Divergent integrals are regularized and evaluated in $n$ dimensions using the technique of dimensional regularization due to 't Hooft and Veltman 40, (see Appendix D).

Following the Feynman rules of Appendix C, the contribution of Fig. 6.6 a to the $\mathrm{Z}$ self-energy tensor is

$$
\begin{aligned}
-i \Sigma_{Z}^{\mu \nu}\left(M_{N}, M_{N}\right) & =-\sum_{a, b=4,5,6} \int \frac{d^{n} q}{(2 \pi)^{n}} \operatorname{Tr}\left\{\frac{i e}{4 s_{w} c_{w}}\left(K_{H}^{\dagger} K_{H}\right)_{a b} \gamma_{\mu}\left(1-\gamma_{5}\right)\right. \\
& \left.\times \frac{i}{\not q-\not p-M_{N}} \frac{i e}{4 s_{w} c_{w}}\left(K_{H}^{\dagger} K_{H}\right)_{b a} \gamma_{\nu}\left(1-\gamma_{5}\right) \frac{i}{\not q-M_{N}}\right\}
\end{aligned}
$$



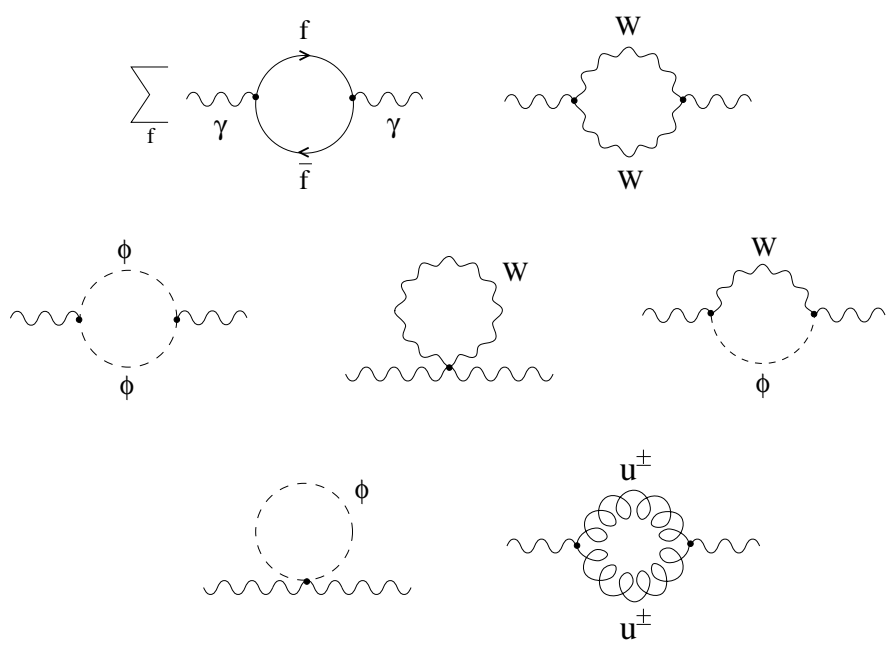

Figure 6.2: Photon self-energy.
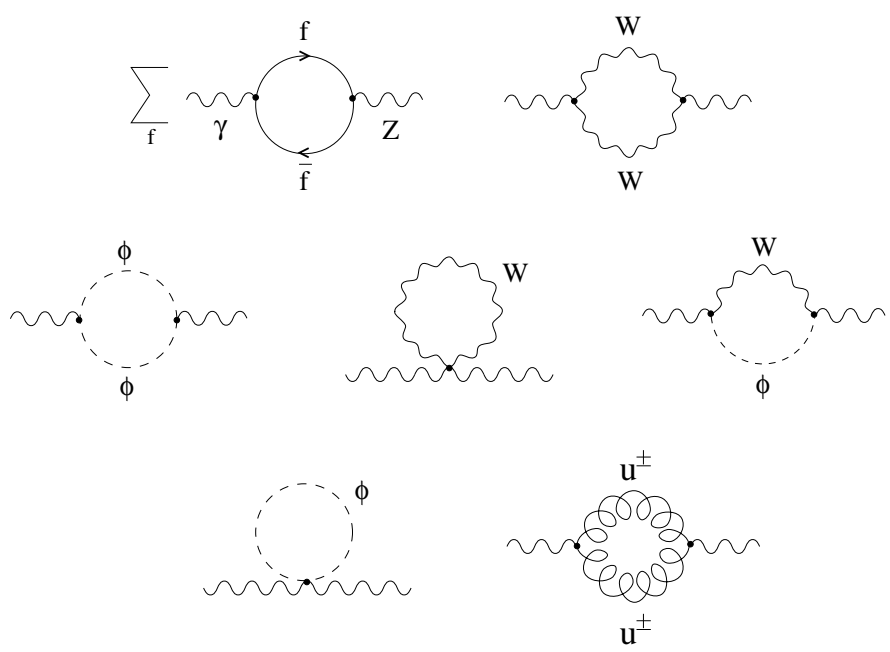

Figure 6.3: Photon-Z mixing energy. 

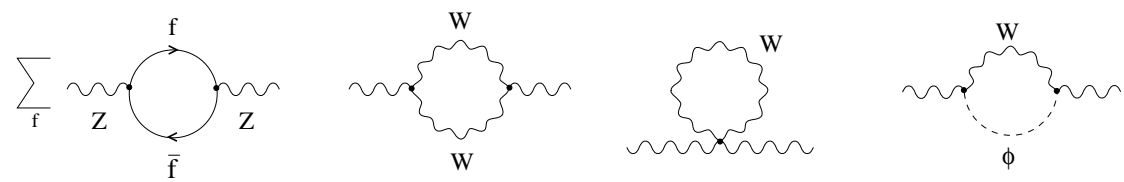

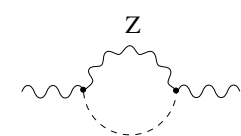

$\mathrm{H}$
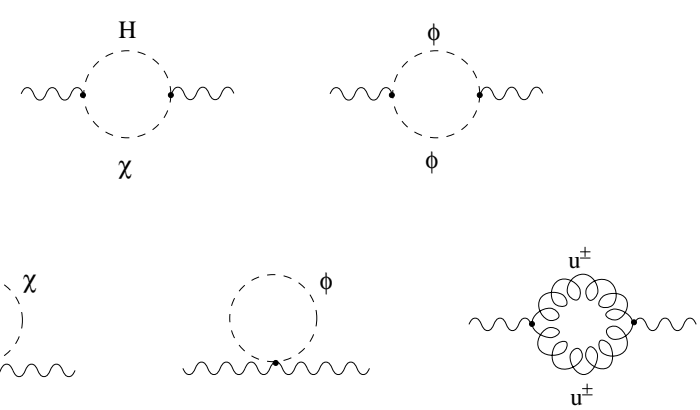

Figure 6.4: Z boson self-energy.
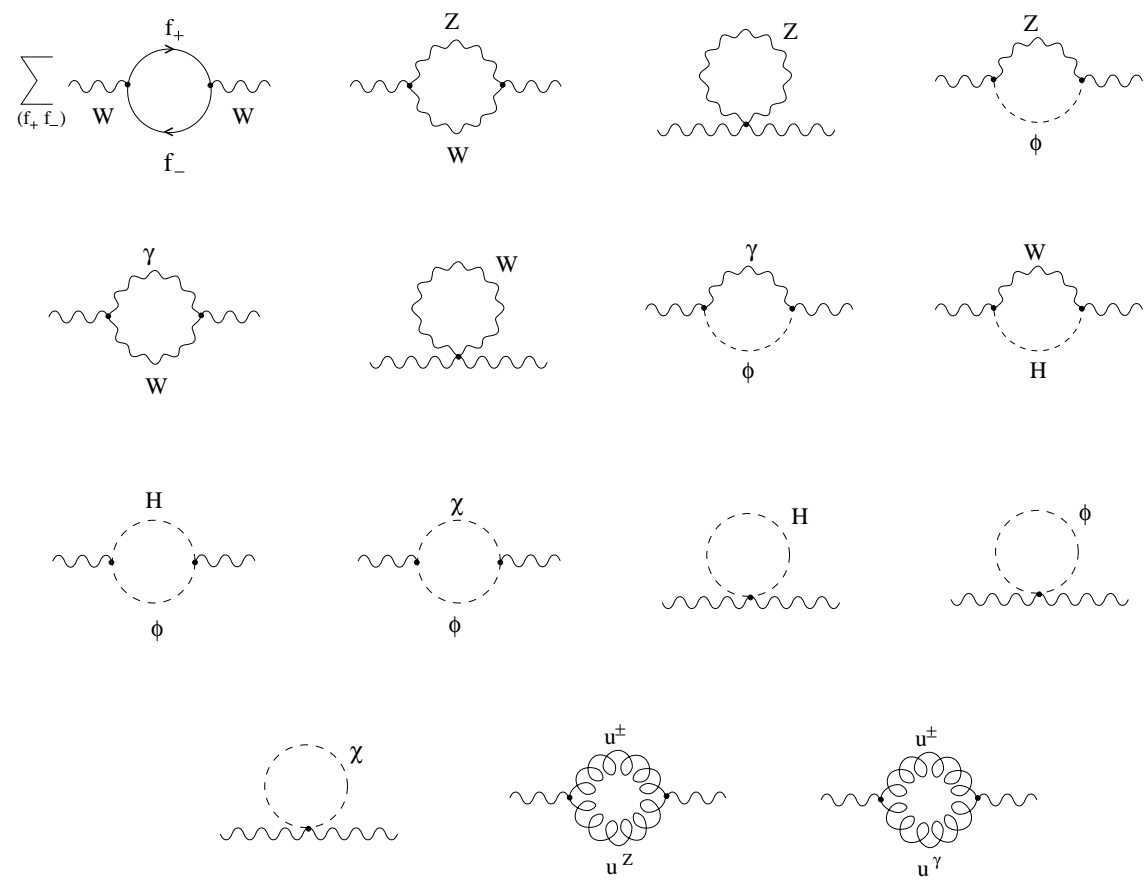

Figure 6.5: W boson self-energy. 


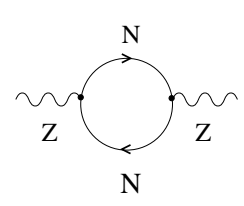

a)

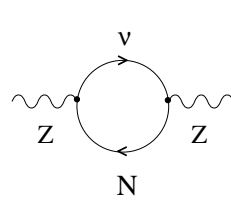

b)

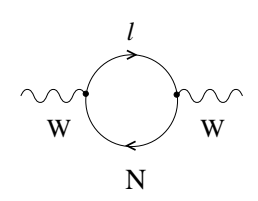

e)

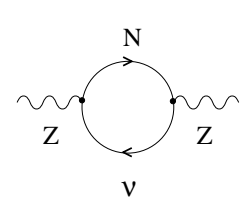

c)

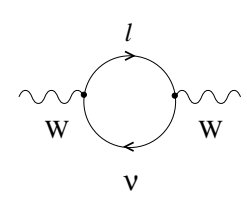

$f)$

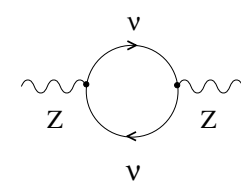

d)

Figure 6.6: Non-SM loops with NHL's and massless neutrinos.

$$
\begin{aligned}
& =-\frac{e^{2}}{16 s_{W}^{2} c_{W}^{2}} \sum_{a, b}\left(K_{H}^{\dagger} K_{H}\right)_{a b}\left(K_{H}^{\dagger} K_{H}\right)_{b a} \int \frac{d^{n} q}{(2 \pi)^{n}} \\
& \times \frac{\operatorname{Tr}\left\{\gamma_{\mu}\left(1-\gamma_{5}\right)\left(\not q-\not p+M_{N}\right) \gamma_{\nu}\left(1-\gamma_{5}\right)\left(\not q+M_{N}\right)\right\}}{\left[(q-p)^{2}-M_{N}^{2}\right]\left[q^{2}-M_{N}^{2}\right]} .
\end{aligned}
$$

In the above we sum over NHL's of all three families $(a, b=4,5,6)$. Using the relations and theorems of Appendix A.1 we now evaluate the trace:

$$
\begin{aligned}
\operatorname{Tr}\{\ldots\} & \equiv \operatorname{Tr}\left\{\gamma_{\mu}\left(1-\gamma_{5}\right)\left(\not q-\not p+M_{N}\right) \gamma_{\nu}\left(1-\gamma_{5}\right)\left(\not q+M_{N}\right)\right\} \\
& =2 \operatorname{Tr}\left\{\gamma_{\mu}\left(1-\gamma_{5}\right)(\not q-\not p) \gamma_{\nu}\left(\not q+M_{N}\right)\right\} \\
& =2 \operatorname{Tr}\left\{\gamma_{\mu}(\not q-\not p) \gamma_{\nu} \not q\right\}-2 \operatorname{Tr}\left\{\gamma_{\mu} \gamma_{5}(\not q-\not p) \gamma_{\nu} \not q\right\}
\end{aligned}
$$


The trace with $\gamma_{5}$ does not contribute:

$$
\operatorname{Tr}\left\{\gamma_{\mu} \gamma_{5}(\not q-\not p) \gamma_{\nu} \not q\right\}=4 i \epsilon_{\mu \alpha \nu \beta}(q-p)^{\alpha} q^{\beta}=0
$$

using $\epsilon_{\mu \alpha \nu \beta} q^{\alpha} q^{\beta}=0$ and $\int_{q} q^{\alpha} \ldots=p^{\alpha} \ldots$. The original trace is thus given by

$$
\begin{aligned}
\operatorname{Tr}\{\ldots\} & =2 \operatorname{Tr}\left\{\gamma_{\mu}(\not q-\not p) \gamma_{\nu} \not q\right\} \\
& =8\left[(q-p)_{\mu} q_{\nu}-g_{\mu \nu}(q-p) q+q_{\mu}(q-p)_{\nu}\right] .
\end{aligned}
$$

We plug this result back in Eq. 6.10:

$$
\begin{aligned}
-i \Sigma_{Z}^{\mu \nu}\left(M_{N}, M_{N}\right) & =-\frac{e^{2}}{2 s_{W}^{2} c_{W}^{2}} k_{H H} \\
& \times \int \frac{d^{n} q}{(2 \pi)^{n}} \frac{(q-p)_{\mu} q_{\nu}-g_{\mu \nu}(q-p) q+q_{\mu}(q-p)_{\nu}}{\left[(q-p)^{2}-M_{N}^{2}\right]\left[q^{2}-M_{N}^{2}\right]} \\
& =-\frac{e^{2}}{2 s_{W}^{2} c_{W}^{2}} k_{H H} \frac{i \pi^{2}}{(2 \pi)^{n}}\left\{-p_{\mu} B_{\nu}\left(p ; M_{N}, M_{N}\right)\right. \\
& -p_{\nu} B_{\mu}\left(p ; M_{N}, M_{N}\right)+g_{\mu \nu} p_{\alpha} B^{\alpha}\left(p ; M_{N}, M_{N}\right) \\
& \left.+2 B_{\mu \nu}\left(p ; M_{N}, M_{N}\right)-g_{\mu \nu} g_{\alpha \beta} B^{\alpha \beta}\left(p ; M_{N}, M_{N}\right)\right\}
\end{aligned}
$$

where $k_{H H} \equiv \sum\left(K_{H}^{\dagger} K_{H}\right)_{a b}\left(K_{H}^{\dagger} K_{H}\right)_{b a}$ and functions $B_{\mu}, B_{\mu \nu}$ are 't Hooft scalar $n$-dimensional integrals defined in Appendix E.3. Using (see Eq. E.14)

$$
B_{\mu}=-p_{\mu} B_{1}, \quad B_{\mu \nu}=p_{\mu} p_{\nu} B_{21}-g_{\mu \nu} B_{22}
$$

the $n$-dimensional space-time relation (see Eq. D.15)

$$
g^{\mu \nu} g_{\mu \nu}=n
$$

and recollecting that only the transverse (terms with $g_{\mu \nu}$ ) part contributes to 
S-matrix elements, we get

$$
\begin{aligned}
-i \Sigma_{Z}^{\mu \nu}\left(M_{N}, M_{N}\right) & =-\frac{e^{2}}{2 s_{W}^{2} c_{W}^{2}} k_{H H} \frac{i \pi^{2}}{(2 \pi)^{n}} g_{\mu \nu}\left[-p^{2} B_{1}-p^{2} B_{21}-2 B_{22}\right. \\
& \left.+n B_{22}\right] .
\end{aligned}
$$

With the help of formulae from Appendix E.3, we arrive at the following result for the self-energy $\Sigma_{Z}\left(M_{N}, M_{N}\right)$ :

$$
\begin{aligned}
-i \Sigma_{Z}\left(M_{N}, M_{N}\right) & =-\frac{e^{2}}{2 s_{W}^{2} c_{W}^{2}} k_{H H} \frac{i \pi^{2}}{(2 \pi)^{n}} \frac{1}{3}\left\{\left(-3 M_{N}^{2}+p^{2}\right) \Delta\right. \\
& \left.+\left[2 A_{0}^{f i n}\left(M_{N}\right)+2 M_{N}^{2}-\frac{p^{2}}{3}+\left(p^{2}-M_{N}^{2}\right) B_{0}^{f i n}\left(p ; M_{N}, M_{N}\right)\right]\right\} \\
& =-\frac{e^{2}}{2 s_{W}^{2} c_{W}^{2}} k_{H H} \frac{i \pi^{2}}{(2 \pi)^{n}} \frac{1}{3}\left\{\left(-3 M_{N}^{2}+p^{2}\right) \Delta\right. \\
& +\left[2 M_{N}^{2} \ln M_{N}^{2}-\frac{p^{2}}{3}+\left(F\left(p ; M_{N}, M_{N}\right)-\ln M_{N}^{2}\right)\right. \\
& \left.\left.\times\left(p^{2}-M_{N}^{2}\right)\right]\right\},
\end{aligned}
$$

where the function $F$ is related to the function $B_{0}$ by Eq. E.17 and $A_{0}^{\text {fin }}(m)=$ $-m^{2}\left(-\ln m^{2}+1\right)$. The divergence is displayed as a pole at $n=4$ (see Appendix D.1):

$$
\Delta=\frac{2}{4-n}-\gamma-\ln \pi=\frac{2}{\epsilon}-\gamma-\ln \pi
$$

In $n$ dimensions, $\alpha$ becomes a dimensional quantity and we should do the the following replacement:

$$
\alpha=\frac{e^{2}}{4 \pi} \rightarrow \alpha \mu^{\epsilon}=\alpha\left(1+\frac{\epsilon}{2} \ln \mu^{2}+O\left(\epsilon^{2}\right)+\ldots\right)
$$


where $\mu$ is an arbitrary mass. Together with the expansion

$$
\frac{1}{(2 \pi)^{n}}=\frac{1}{(2 \pi)^{4-\epsilon}}=\frac{1}{(2 \pi)^{4}}\left(1+\epsilon \ln 2 \pi+O\left(\epsilon^{2}\right)+\ldots\right)
$$

this yields

$$
\begin{aligned}
\frac{1}{(2 \pi)^{n}} \alpha \Delta & =\frac{1}{(2 \pi)^{4}} \alpha\left(\frac{2}{\epsilon}-\gamma+\ln 4 \pi+\ln \mu^{2}\right)=\frac{1}{(2 \pi)^{4}} \alpha \Delta_{\mu} \\
& =\frac{1}{(2 \pi)^{4}} \alpha\left(\Delta_{m}+\ln m^{2}\right), \quad \Delta_{m}=\frac{2}{\epsilon}-\gamma+\ln 4 \pi+\ln \frac{\mu^{2}}{m^{2}}
\end{aligned}
$$

The self-energy thus becomes

$$
\begin{aligned}
\Sigma_{Z}\left(M_{N}, M_{N}\right) & =\frac{\alpha}{8 \pi} \frac{1}{s_{W}^{2} c_{W}^{2}} k_{H H} \frac{1}{3}\left\{\left(-3 M_{N}^{2}+p^{2}\right)\left(\Delta_{M_{N}}+\ln M_{N}^{2}\right)\right. \\
& \left.+\left[2 M_{N}^{2} \ln M_{N}^{2}-\frac{p^{2}}{3}+\left(F\left(p ; M_{N}, M_{N}\right)-\ln M_{N}^{2}\right)\left(p^{2}-M_{N}^{2}\right)\right]\right\} \\
& =\frac{\alpha}{8 \pi} \frac{1}{s_{W}^{2} c_{W}^{2}} k_{H H}\left\{\Delta_{M_{N}}\left(\frac{p^{2}}{3}-M_{N}^{2}\right)-\frac{p^{2}}{9}\right. \\
& \left.+\frac{1}{3} F\left(p ; M_{N}, M_{N}\right)\left(p^{2}-M_{N}^{2}\right)\right\}
\end{aligned}
$$

For the other three contributions we get, following the same steps,

$$
\begin{aligned}
\Sigma_{Z}\left(M_{N}, 0\right) & =\frac{\alpha}{8 \pi} \frac{1}{s_{W}^{2} c_{W}^{2}} k_{H L}\left\{\Delta_{M_{N}}\left(\frac{p^{2}}{3}-\frac{M_{N}^{2}}{2}\right)+\frac{2}{9} p^{2}-\frac{M_{N}^{2}}{6}\right. \\
& \left.+F\left(p ; M_{N}, 0\right)\left(\frac{p^{2}}{3}-\frac{M_{N}^{2}}{6}-\frac{M_{N}^{4}}{6 p^{2}}\right)\right\}, \\
\Sigma_{Z}\left(0, M_{N}\right) & =\Sigma_{Z}\left(M_{N}, 0\right), \\
\Sigma_{Z}(0,0) & =\frac{\alpha}{8 \pi} \frac{1}{s_{W}^{2} c_{W}^{2}} k_{L L} \frac{p^{2}}{3}\left(\Delta_{m}+F(p ; m, m)-\frac{1}{3}\right),
\end{aligned}
$$

where $m^{2} \ll p^{2}$, otherwise $m$ can be arbitrary since $F(p ; m, m)=1-\ln \left(-p^{2} / m^{2}\right.$ - $i \epsilon)$ and therefore $\Delta_{m}+F(p ; m, m)$ is independent of $m$. The mixing factors $k_{H H}, k_{H L}$ and $k_{L L}$ can be cast into a more convenient form by converting $K_{L}$ matrices 
into $K_{H}$ matrices with the help of Eq. 3.24:

$$
\begin{aligned}
k_{H H}= & \sum_{a, b}\left(K_{H}^{\dagger} K_{H}\right)_{a b}\left(K_{H}^{\dagger} K_{H}\right)_{b a}=\sum_{a, b, l, j}\left(K_{H}^{\dagger}\right)_{a l}\left(K_{H}\right)_{l b}\left(K_{H}^{\dagger}\right)_{b j}\left(K_{H}\right)_{j a} \\
= & \sum_{a, b, l, j}\left(K_{H}^{*}\right)_{l a}\left(K_{H}\right)_{j a}\left(K_{H}\right)_{l b}\left(K_{H}^{*}\right)_{j b}=e e_{m i x}^{2}+\left|e \mu_{m i x}\right|^{2}+\left|e \tau_{m i x}\right|^{2} \\
& +\left|e \mu_{m i x}\right|^{2}+\mu \mu_{m i x}^{2}+\left|\mu \tau_{m i x}\right|^{2}+\left|e \tau_{m i x}\right|^{2}+\left|\mu \tau_{m i x}\right|^{2}+\tau \tau_{m i x}^{2}, \\
k_{H L}= & \sum_{a, i}\left(K_{H}^{\dagger} K_{L}\right)_{a i}\left(K_{L}^{\dagger} K_{H}\right)_{i a}=\sum_{a, i, j, k}\left(K_{H}^{\dagger}\right)_{a j}\left(K_{L}\right)_{j i}\left(K_{L}^{\dagger}\right)_{i k}\left(K_{H}\right)_{k a} \\
= & \sum_{a, j, k}\left(K_{H}^{\dagger}\right)_{a j} \delta_{j k}\left(K_{H}\right)_{k a}-\sum_{a, b, j, k}\left(K_{H}^{\dagger}\right)_{a j}\left(K_{H}\right)_{j b}\left(K_{H}^{\dagger}\right)_{b k}\left(K_{H}\right)_{k a} \\
= & \sum_{a, k}\left(K_{H}^{\dagger}\right)_{a k}\left(K_{H}\right)_{k a}-\sum_{a, b}\left(K_{H}^{\dagger} K_{H}\right)_{a b}\left(K_{H}^{\dagger} K_{H}\right)_{b a} \\
= & e e_{m i x}+\mu \mu_{m i x}+\tau \tau_{m i x}-k_{H H}, \\
k_{L L}= & \sum_{i, j}\left(K_{L}^{\dagger} K_{L}\right)_{j i}\left(K_{L}^{\dagger} K_{L}\right)_{i j}=\sum_{i, j, k, l}\left(K_{L}^{\dagger}\right)_{j k}\left(K_{L}\right)_{k i}\left(K_{L}^{\dagger}\right)_{i l}\left(K_{L}\right)_{l j} \\
= & \ldots=3-2\left(e e_{m i x}+\mu \mu_{m i x}+\tau \tau_{m i x}\right)+k_{H H} .
\end{aligned}
$$

The total Z self-energy in our model is obtained by cutting out the neutrino contribution from the total Z self-energy in the SM (the first line of Eq. E.7) and replacing it with the sum $\Sigma_{Z}\left(M_{N}, M_{N}\right)+2 \Sigma_{Z}\left(M_{N}, 0\right)+\Sigma_{Z}(0,0)$.

The W self-energy calculation goes along the same lines yielding

$$
\begin{aligned}
\Sigma_{W}\left(M_{N}, m_{l}\right) & =\frac{\alpha}{12 \pi s_{W}^{2}}\left\{\sum _ { l = e , \mu , \tau } l l _ { m i x } \left[\frac{\Delta^{M_{N}}}{2}\left(p^{2}-\frac{5}{2} M_{N}^{2}-\frac{m_{l}^{2}}{2}\right)\right.\right. \\
& +\frac{\Delta^{m_{l}}}{2}\left(p^{2}-\frac{5}{2} m_{l}^{2}-\frac{M_{N}^{2}}{2}\right) \\
& +\left(p^{2}-\frac{M_{N}^{2}+m_{l}^{2}}{2}-\frac{\left(M_{N}^{2}-m_{l}^{2}\right)^{2}}{2 p^{2}}\right) F\left(p ; M_{N}, m_{l}\right) \\
& \left.\left.+\left(p^{2}-\frac{M_{N}^{2}+m_{l}^{2}}{2}\right)\left(1-\frac{M_{N}^{2}+m_{l}^{2}}{M_{N}^{2}-m_{l}^{2}} \ln \frac{M_{N}}{m_{l}}\right)-\frac{p^{2}}{3}\right]\right\}
\end{aligned}
$$




$$
\begin{aligned}
\Sigma_{W}\left(0, m_{l}\right) & =\frac{\alpha}{12 \pi s_{W}^{2}}\left\{\sum _ { l = e , \mu , \tau } ( 1 - l l _ { m i x } ) \left[\left(p^{2}-\frac{3}{2} m_{l}^{2}\right) \Delta^{m_{l}}\right.\right. \\
& \left.\left.+\left(p^{2}-\frac{m_{l}^{2}}{2}-\frac{m_{l}^{4}}{2 p^{2}}\right) F\left(p ; 0, m_{l}\right)+\frac{2}{3} p^{2}-\frac{m_{l}^{2}}{2}\right]\right\},
\end{aligned}
$$

for the diagrams of Figs. 6.6e,f respectively. The total W self-energy in our model is obtained by cutting out the lepton contribution from the total $\mathrm{W}$ self-energy in the SM (the first two lines of Eq. E.8) and replacing it with the sum $\Sigma_{W}\left(M_{N}, m_{l}\right)+$ $\Sigma_{W}\left(0, m_{l}\right)$. The self-energies are then renormalized using (see Eq. E.2)

$$
\begin{aligned}
& \hat{\Sigma}_{Z}\left(p^{2}\right)=\Sigma_{Z}\left(p^{2}\right)-\delta M_{Z}^{2}+\delta Z_{2}^{Z}\left(p^{2}-M_{Z}^{2}\right), \\
& \hat{\Sigma}_{W}\left(p^{2}\right)=\Sigma_{W}\left(p^{2}\right)-\delta M_{W}^{2}+\delta Z_{2}^{W}\left(p^{2}-M_{W}^{2}\right),
\end{aligned}
$$

with renormalization constants given by Eq. E.4. Note the form of the equations above is the same as in the SM.

In order to better see the dependence of $\hat{\Pi}_{Z}$ on $M_{N}$ for $M_{N} \gg M_{W}$ (this is the limit we are ultimately interested in, see Sec. 6.6.), we split $\hat{\Pi}_{Z}$ as

$$
\hat{\Pi}_{Z}=\hat{\Pi}_{Z}^{S M}+\hat{\Pi}_{Z}^{N H L}
$$

where $\hat{\Pi}_{Z}^{S M}$ is the SM limit of $\hat{\Pi}_{Z}$ and $\hat{\Pi}_{Z}^{N H L}$ are corrections due to NHL's. Expanding the $F$ functions in powers of $M_{N}^{2}$, we obtain in the limit of $M_{N} \gg M_{W}$

$$
\hat{\Pi}_{Z}^{N H L}=\frac{\alpha}{\pi}\left\{\frac{c_{W}^{2}-s_{W}^{2}}{16 s_{W}^{4}} \frac{M_{N}^{2}}{M_{W}^{2}} k_{H H}+O\left(\ln M_{N}^{2} / M_{W}^{2}\right)+\ldots\right\} .
$$

Although this formula looks very simple, one should be aware of one important fact. The leading term is suppressed by the mixing parameter. Indeed, the $k_{H H}$ mixing is quadratic in $\tau \tau_{m i x}$, while some of the $O\left(\ln M_{N}^{2} / M_{W}^{2}\right)$ terms are only linear. As a result, a few of them are comparable in size to the leading term in $M_{N}^{2}$ expansion up to $\sim 1 \mathrm{TeV}$ NHL mass. We illustrate this point in Table 6.1. Here we show 
numerical predictions for the (exact) oblique parameter $\hat{\Pi}_{Z}$ and compare them with the approximate parameter $\hat{\Pi}_{Z a p p x}=\hat{\Pi}_{Z}^{S M}+\hat{\Pi}_{Z a p p x}^{N H L}$ where $\hat{\Pi}_{Z a p p x}^{N H L}$ is the first term in Eq. 6.30 ษ. The contribution of higher order terms from Eq. 6.30 is given by the difference $d=\hat{\Pi}_{Z}-\hat{\Pi}_{Z a p p x}$. Input numbers used are $M_{Z}=91.1884 \mathrm{GeV}$, $M_{H}=200 \mathrm{GeV}, m_{t}=176 \mathrm{GeV}, \tau \tau_{m i x}=0.033$ and $e e_{m i x}=\mu \mu_{\text {mix }}=0$.

\begin{tabular}{|l|r|r|r|r|l|}
\hline$M_{N}$ & $0.5 \mathrm{TeV}$ & $1 \mathrm{TeV}$ & $3 \mathrm{TeV}$ & $5 \mathrm{TeV}$ & \\
\hline$\hat{\Pi}_{Z}^{S M}$ & -4.299 & -4.297 & -4.265 & -4.199 & $\times 10^{-2}$ \\
$\hat{\Pi}_{Z}$ & -4.313 & -4.298 & -4.054 & -3.526 & $\times 10^{-2}$ \\
$\hat{\Pi}_{Z \text { appx }}=\hat{\Pi}_{Z}^{S M}+\hat{\Pi}_{Z \text { app }}^{N H L}$ & -4.292 & -4.270 & -4.013 & -3.479 & $\times 10^{-2}$ \\
$\hat{\Pi}_{Z \text { app }}^{N H L}$ & 0.007 & 0.027 & 0.252 & 0.720 & $\times 10^{-2}$ \\
$d=\hat{\Pi}_{Z}-\hat{\Pi}_{Z a p p x}$ & -0.021 & -0.028 & -0.041 & -0.047 & $\times 10^{-2}$ \\
\hline
\end{tabular}

Table 6.1: Comparison of $\hat{\Pi}_{Z a p p x}$ with $\hat{\Pi}_{Z}$

For $M_{N}=0.5 \mathrm{TeV}, \hat{\Pi}_{Z a p p x}^{N H L}$ contributes $0.007 \times 10^{-2}$ of the total difference between $\hat{\Pi}_{Z}$ and $\hat{\Pi}_{Z}^{S M}\left(\hat{\Pi}_{Z}-\hat{\Pi}_{Z}^{S M}=\mathrm{d}+\hat{\Pi}_{Z \text { appx }}^{N H L}\right)$. The higher order terms contribute more (with opposite sign), $d=-0.021 \times 10^{-2}$. At $1 \mathrm{TeV}, \hat{\Pi}_{Z a p p x}^{N H L}=0.027 \times 10^{-2}$ is comparable with $|d|=0.028 \times 10^{-2}$ and at $3 \mathrm{TeV}$ it already dominates.

Overall, $\hat{\Pi}_{Z a p p x}$ differs from $\hat{\Pi}_{Z}$ by approximately $1 \%$ in the considered range of NHL masses.

\subsection{Vertex factor $\delta \hat{\Gamma}_{l l}$}

The vertex factor

$$
\delta \hat{\Gamma}_{l l}=\frac{2}{3} \alpha M_{Z}\left\{v_{l}\left[\mathcal{R} e \hat{F}_{V}\left(M_{Z}^{2}\right)-\hat{\Pi}_{\gamma Z}\left(M_{Z}^{2}\right)\right]+a_{l} \mathcal{R} e \hat{F}_{A}\left(M_{Z}^{2}\right)\right\}
$$

\footnotetext{
${ }^{1}$ The dependence of $\hat{\Pi}_{Z}^{S M}$ on the NHL mass has its origin in a different value of the input parameter $M_{W}$ (as calculated from $G_{\mu}$, see Sec. 6.5) for different NHL masses. Thus the formula for $\hat{\Pi}_{Z}^{S M}$ comes from the SM, but the choice of $M_{W}$ comes from our model, not the SM.
} 
is the source of the lepton universality breaking in $\Gamma_{l l}$. It includes, besides irreducible vertex corrections, also lepton wave function renormalization and the renormalized mixing energy $\hat{\Sigma}_{\gamma Z}$. The irreducible vertices (Fig. 6.7) and lepton wave function corrections (Fig. 6.8) are absorbed in the renormalized formfactors $\hat{F}_{V}, \hat{F}_{A}$ while the mixing energy (Fig. 6.3) comes in as

$$
\hat{\Pi}_{\gamma Z}\left(p^{2}\right)=\frac{\mathcal{R} e \hat{\Sigma}_{\gamma Z}\left(p^{2}\right)}{p^{2}}
$$

As in the case of $\hat{\Sigma}_{Z}, \hat{\Sigma}_{\gamma Z}$ (given in Appendix E.1) includes besides $\Sigma_{\gamma Z}$ also $\Sigma_{W}$ and $\Sigma_{Z}$ (through the renormalization constants $\delta Z_{1}^{\gamma Z}, \delta Z_{2}^{\gamma Z}$ ). This is the source of the $M_{N}$ dependence of $\hat{\Sigma}_{\gamma Z}$, in spite of $\Sigma_{\gamma Z}$ being a purely SM quantity.

With $\Sigma_{W}, \Sigma_{Z}$ calculated in Sec. 6.3 we get in the limit of $M_{N} \gg M_{W}$ in the leading order an expression similar to Eq. 6.30:

$$
\hat{\Pi}_{\gamma Z}\left(M_{Z}^{2}\right)=\hat{\Pi}_{\gamma Z}\left(M_{Z}^{2}\right)^{S M}-\frac{\alpha}{\pi}\left\{\frac{c_{W}}{16 s_{W}^{3}} \frac{M_{N}^{2}}{M_{W}^{2}} k_{H H}+O\left(\ln \frac{M_{N}^{2}}{M_{W}^{2}}\right)+\ldots\right\}
$$

\subsubsection{Irreducible vertices}

Here we examine irreducible vertices. As an example we calculate the contribution to the unrenormalized form factors $F_{V}, F_{A}$ of the diagram of Fig. 6.7f, which we redraw in Fig. 6.9 to show our convention of momenta flow $\left(p_{1}, p_{2}, p=p_{1}+p_{2}, q\right)$ and charge flow (arrows on internal lines). 


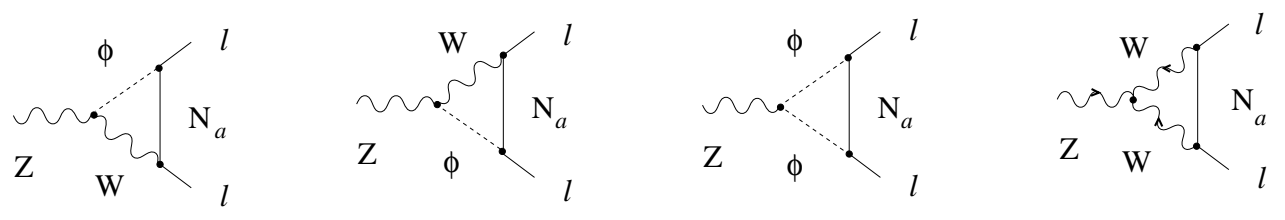

a)

b)

c)

d)

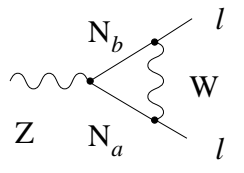

e)

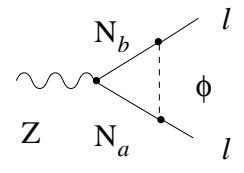

f)

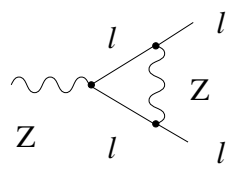

g)

Figure 6.7: Irreducible vertex corrections.

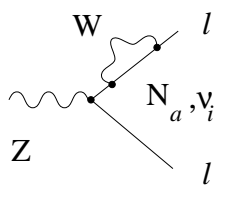

a)

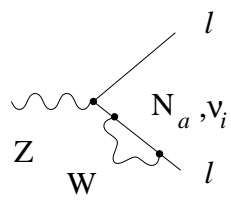

b)

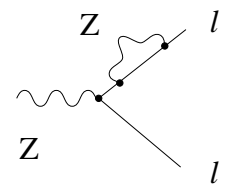

e)

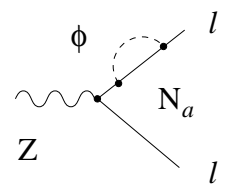

c)

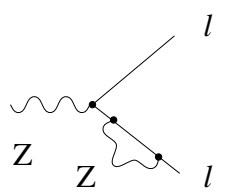

f)

Figure 6.8: Lepton self-energies. 


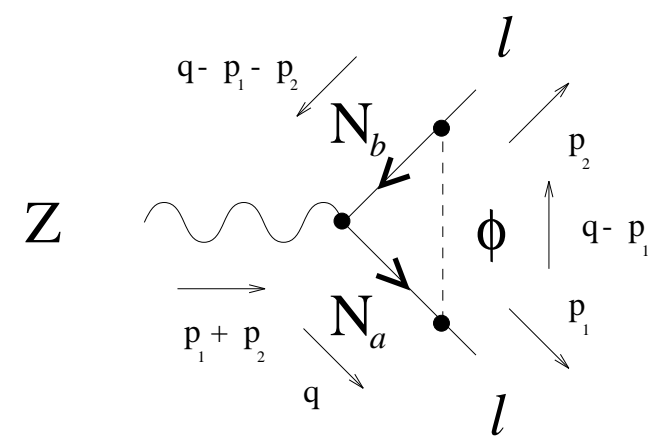

Figure 6.9: Momenta and charge flow convention.

Following the Feynman rules of Appendix Q, the vertex $V_{N N \phi}^{\mu}$ is given by (terms $m_{l}^{2} / M_{W}^{2}$ neglected)

$$
\begin{aligned}
V_{N N \phi}^{\mu} & =\sum_{a, b} \int \frac{d^{n} q}{(2 \pi)^{n}} \frac{i}{\left(q-p_{1}\right)^{2}-M_{W}^{2}} \frac{+i g_{2}}{\sqrt{2} M_{W}}\left(K_{H}\right)_{l a} M_{N} \frac{1+\gamma_{5}}{2} \frac{i}{\not q-M_{N}} \\
& \times \frac{+i e}{4 s_{W} c_{W}}\left(K_{H}^{\dagger} K_{H}\right)_{a b} \gamma_{\mu}\left(1-\gamma_{5}\right) \frac{i}{\not q-\not p_{1}-\not p_{2}-M_{N}} \frac{+i g_{2}}{\sqrt{2} M_{W}}\left(K_{H}^{\dagger}\right)_{b l} M_{N} \\
& \times \frac{1-\gamma_{5}}{2} .
\end{aligned}
$$

Introducing a shorthand $l_{2} \equiv \sum\left(K_{H}\right)_{l a}\left(K_{H}^{\dagger} K_{H}\right)_{a b}\left(K_{H}^{\dagger}\right)_{b l}$, collecting numerical factors at the front and merging $1 \pm \gamma_{5}$ factors we get

$$
\begin{aligned}
V_{N N \phi}^{\mu} & =-\frac{e^{3}}{32 s_{W}^{3} c_{W}} \frac{M_{N}^{2}}{M_{W}^{2}} l_{2} \\
& \times \int \frac{d^{n} q}{(2 \pi)^{n}} \frac{4 M_{N}^{2} \gamma^{\mu}\left(1-\gamma_{5}\right)}{\left(q^{2}-M_{N}^{2}\right)\left[\left(q-p_{1}\right)^{2}-M_{W}^{2}\right]\left[\left(q-p_{1}-p_{2}\right)^{2}-M_{N}^{2}\right]}
\end{aligned}
$$

Now we can identify the integral as a finite $C_{0}$ function (see Eq. E.9):

$$
V_{N N \phi}^{\mu}=-\frac{e^{3}}{8 s_{W}^{3} c_{W}} \frac{M_{N}^{4}}{M_{W}^{2}} l_{2} \frac{-i \pi^{2}}{(4 \pi)^{2} \pi^{2}} C_{0}\left(M_{N}, M_{W}, M_{N}\right) \gamma^{\mu}\left(1-\gamma_{5}\right)
$$




$$
\begin{aligned}
& =+i e \gamma^{\mu}\left(1-\gamma_{5}\right) \frac{\alpha}{4 \pi} l_{2} \frac{M_{W}^{2}}{8 s_{W}^{3} c_{W}} \frac{M_{N}^{4}}{M_{W}^{4}} C_{0}\left(M_{N}, M_{W}, M_{N}\right) \\
& =+i e \gamma^{\mu}\left(1-\gamma_{5}\right) \frac{\alpha}{4 \pi} l_{2} \mathcal{M}_{N N \phi} .
\end{aligned}
$$

The total contribution of the irreducible vertex diagrams and the definition of the unrenormalized form factors $F_{V}, F_{A}$ is given by

$$
\begin{aligned}
V^{\mu} & =+i e \gamma^{\mu} F_{V}-i e \gamma^{\mu} \gamma_{5} F_{A} \\
& =+i e \gamma^{\mu} \frac{\alpha}{4 \pi}\left\{l_{1} \mathcal{M}_{\phi W N}+l_{2} \mathcal{M}_{N N \phi}+l_{1} \mathcal{M}_{\phi \phi N}-\left(1-l_{1}\right) \mathcal{M}_{W W \nu}\right. \\
& \left.+l_{1} \mathcal{M}_{W W N}+l_{3} \mathcal{M}_{N \nu W}+l_{3} \mathcal{M}_{\nu N W}+l_{4} \mathcal{M}_{\nu \nu W}+l_{2} \mathcal{M}_{N N W}-\left(c_{L}^{3}+c_{R}^{3}\right) \mathcal{M}_{l l Z}\right\} \\
& -i e \gamma^{\mu} \gamma_{5} \frac{\alpha}{4 \pi}\left\{l_{1} \mathcal{M}_{\phi W N}+l_{2} \mathcal{M}_{N N \phi}+l_{1} \mathcal{M}_{\phi \phi N}-\left(1-l_{1}\right) \mathcal{M}_{W W \nu}+l_{1} \mathcal{M}_{W W N}\right. \\
& \left.+l_{3} \mathcal{M}_{N \nu W}+l_{3} \mathcal{M}_{\nu N W}+l_{4} \mathcal{M}_{\nu \nu W}+l_{2} \mathcal{M}_{N N W}-\left(c_{L}^{3}-c_{R}^{3}\right) \mathcal{M}_{l l Z}\right\},
\end{aligned}
$$

where

$$
\begin{aligned}
c_{L} & =-\frac{1}{2}+s_{W}^{2}, \quad c_{R}=s_{W}^{2} \\
\mathcal{M}_{l l Z} & =+\frac{1}{2 s_{W}^{3} c_{W}^{3}}\left[2 M_{Z}^{2}\left(C_{23}\left(m_{l}, M_{Z}, m_{l}\right)+C_{11}\left(m_{l}, M_{Z}, m_{l}\right)\right)+2\right. \\
& \left.-4 C_{24}^{f i n}\left(m_{l}, M_{Z}, m_{l}\right)-\Delta_{\mu}\right]
\end{aligned}
$$

$\Delta_{\mu}$ is given in Eq. D.9 and $\mathcal{M}_{\phi W N}, \ldots$ were defined before (see Eq. 5.2). The mixing factors are obtained from the flavour-violating ones (see Eq. 5.4) by setting $l=l^{\prime}$ :

$$
\begin{aligned}
& l_{1}=\left(K_{H}\right)_{l a}\left(K_{H}^{\dagger}\right)_{a l}=l l_{\text {mix }}, \\
& l_{2}=\left(K_{H}\right)_{l a}\left(K_{H}^{\dagger} K_{H}\right)_{a b}\left(K_{H}^{\dagger}\right)_{b l}=\left|l e_{m i x}\right|^{2}+\left|l \mu_{m i x}\right|^{2}+\left|l \tau_{m i x}\right|^{2}, \\
& l_{3}=\left(K_{L}\right)_{l i}\left(K_{L}^{\dagger} K_{H}\right)_{i a}\left(K_{H}^{\dagger}\right)_{a l}=l_{1}-l_{2}, \\
& l_{4}=\left(K_{L}\right)_{l i}\left(K_{L}^{\dagger} K_{L}\right)_{i j}\left(K_{L}^{\dagger}\right)_{j l}=1-2 l_{1}+l_{2} .
\end{aligned}
$$


Renormalized form factors $\hat{F}_{V}, \hat{F}_{A}$ are defined (using Eq. E.3) as

$$
\begin{aligned}
\hat{V}^{\mu} & =+i e \gamma^{\mu} \hat{F}_{V}-i e \gamma^{\mu} \gamma_{5} \hat{F}_{A} \\
& =+i e \gamma^{\mu}\left\{F_{V}+v_{l}\left(\delta Z_{1}^{Z}-\delta Z_{2}^{Z}\right)-\left(\delta Z_{1}^{\gamma Z}-\delta Z_{2}^{\gamma Z}\right)+\left(v_{l} \delta Z_{V}^{l}+a_{l} \delta Z_{A}^{l}\right)\right\} \\
& -i e \gamma^{\mu} \gamma_{5}\left\{F_{A}+a_{l}\left(\delta Z_{1}^{Z}-\delta Z_{2}^{Z}\right)+\left(v_{l} \delta Z_{A}^{l}+a_{l} \delta Z_{V}^{l}\right)\right\} .
\end{aligned}
$$

The check of the cancellation of divergences in $\hat{V}^{\mu}$ shows that the divergence of Fig. 6.7c is cancelled by those of Figs. 6.8c,d and the divergence of Fig. 6.7g by those of Figs. 6.8e,f. The sum of the remaining divergences of Figs. 6.7d,e is cancelled by the sum of the divergences of Figs. 6.8 a,b plus those associated with the counterterms $\delta Z_{1}^{Z}-\delta Z_{2}^{Z}$ and $\delta Z_{1}^{\gamma Z}-\delta Z_{2}^{\gamma Z}$ (these come from the bosonic loops of the photon-Z mixing energy).

\subsubsection{Lepton self-energies}

We define lepton self-energies $\Sigma^{l}$ at the one-loop level as shown in Fig. 6.10 , i.e.,

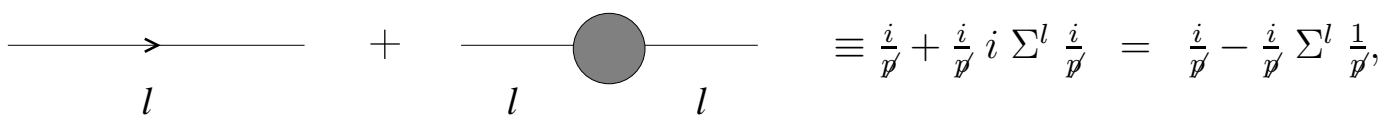

Figure 6.10: The definition of the lepton self-energy $\Sigma^{l}$

we call the blob $i \Sigma^{l}$, rather than $-i \Sigma^{l}$ (Hollik's convention). $\Sigma^{l}$ has the form

$$
\begin{aligned}
\Sigma^{l}(p) & =\not p \Sigma_{V}^{l}\left(p^{2}\right)+\not p \gamma_{5} \Sigma_{A}^{l}\left(p^{2}\right)+m_{l} \Sigma_{S}^{l}\left(p^{2}\right) \\
& =\not p \frac{1-\gamma_{5}}{2} \Sigma_{L}^{l}\left(p^{2}\right)+\not p \frac{1+\gamma_{5}}{2} \Sigma_{R}^{l}\left(p^{2}\right)+m_{l} \Sigma_{S}^{l}\left(p^{2}\right),
\end{aligned}
$$


where $\sum_{V, A, S, L, R}^{l}\left(p^{2}\right)$ are vector, axial vector, scalar, left-handed and right-handed parts respectively. Renormalized charged lepton self-energies $\hat{\Sigma}^{l}$ (see Eq. E.2) do not contribute to the renormalized vector and axial vector formfactors $\hat{F}_{V}, \hat{F}_{A}$. Indeed, the graphs of Fig. 6.8 give zero after the on-shell renormalization conditions (see Eq. 4.48) are applied. However, the unrenormalized lepton self-energies $\Sigma^{l}$ do contribute to the formfactors $\hat{F}_{V}, \hat{F}_{A}$ through the renormalization constants $\delta Z_{V}^{l}, \delta Z_{A}^{l}$. The relation between these renormalization constants and $\Sigma^{l}$ can be found in Eq. E.4.

The charged lepton self-energy is the sum of four parts corresponding to loops with $W-N, W-\nu, \phi-N$ and $Z-l$ respectively (see Fig. 6.8):

$$
\Sigma^{l}(p)=\Sigma_{W N}^{l}+\Sigma_{W \nu}^{l}+\Sigma_{\phi N}^{l}+\Sigma_{Z l}^{l},
$$

where

$$
\begin{aligned}
\Sigma_{W N}^{l} & =-\frac{\alpha}{32 \pi s_{W}^{2}} l_{1}\left[1-2 \Delta_{\mu}+2 \ln M_{W}^{2}+2 f(\mathcal{X})\right] \not p\left(1-\gamma_{5}\right), \\
\Sigma_{W \nu}^{l} & =-\frac{\alpha}{32 \pi s_{W}^{2}}\left(1-l_{1}\right)\left[1-2 \Delta_{\mu}+2 \ln M_{W}^{2}\right] \not p\left(1-\gamma_{5}\right), \\
\Sigma_{\phi N}^{l} & =+\frac{\alpha}{32 \pi s_{W}^{2}} l_{1} \mathcal{X}\left[\Delta_{\mu}+\frac{1}{2}-\ln M_{W}^{2}-f(\mathcal{X})\right] \not p\left(1-\gamma_{5}\right), \\
\Sigma_{Z l}^{l} & =-\frac{\alpha}{16 \pi s_{W}^{2} c_{W}^{2}} c_{L}^{2}\left[1-2 \Delta_{\mu}+2 \ln M_{Z}^{2}\right] \not p\left(1-\gamma_{5}\right) \\
& -\frac{\alpha}{16 \pi s_{W}^{2} c_{W}^{2}} c_{R}^{2}\left[1-2 \Delta_{\mu}+2 \ln M_{Z}^{2}\right] \not p\left(1+\gamma_{5}\right), \\
f(\mathcal{X}) & =\frac{\mathcal{X}^{2}}{(\mathcal{X}-1)^{2}} \ln \mathcal{X}+\frac{\mathcal{X}}{1-\mathcal{X}}, \quad \mathcal{X}=\frac{M_{N}^{2}}{M_{W}^{2}} .
\end{aligned}
$$

These expressions are evaluated at $p^{2}=m_{l}^{2}$ as required by $\delta Z_{V}^{l}, \delta Z_{A}^{l}$ (see Eq. E.4), and terms $\frac{m_{l}^{2}}{M_{W}^{2}}$ were neglected. Note the $\Sigma_{Z l}^{l}$ part is a pure SM result. For completeness, we also give $\Sigma_{\gamma l}^{l}$ :

$$
\Sigma_{\gamma l}^{l}=\frac{\alpha}{4 \pi}\left[\Delta_{\mu}-1+2 B_{0}^{f i n}\left(p ; m_{\lambda}, m_{l}\right)+2 B_{1}^{f i n}\left(p ; m_{\lambda}, m_{l}\right)\right] \not p
$$




$$
-\frac{\alpha}{\pi}\left[\Delta_{\mu}-\frac{1}{2}+B_{0}^{f i n}\left(p ; m_{\lambda}, m_{l}\right)\right] m_{l}
$$

Here $m_{\lambda}$ is the regularized photon mass and $B_{0}^{f i n}, B_{1}^{f i n}$ (evaluated at $p^{2}=m_{l}^{2}$ ) are given in Eq. E.19. $\Sigma_{\gamma l}^{l}$ is, in this case, part of the QED subset treated independently of genuine electroweak corrections (see Sec. 6.2). However, in Chapter 7, this photonic correction will be included as a part of the total lepton self-energy and the counterterm $\delta Z_{L}^{l}$ together with the genuine electroweak corrections.

\subsubsection{Form factors $\hat{F}_{V}, \hat{F}_{A}$ in the limit of large NHL mass}

Studying the behaviour of $\hat{F}_{V}, \hat{F}_{A}$ in the limit of $M_{N} \gg M_{W}$, we observe in agreement with Sec. 5.1.2 the three sources of quadratic nondecoupling: $\mathcal{M}_{N N \phi}, \mathcal{M}_{\phi \phi N}$ and $\mathcal{M}_{\phi N}$ (which contains $\Sigma_{\phi N}^{l}$ ). The quadratic nondecoupling of these amplitudes is also expected from dimensional analysis considerations. For illustration, for $\mathcal{M}_{N N \phi}$ we get from Eq. 6.35

$$
\mathcal{M}_{N N \phi} \sim M_{N}^{4} \int \frac{d^{n} q}{\left(q^{2}-M_{N}^{2}\right)\left[\left(q-p_{1}\right)^{2}-M_{W}^{2}\right]\left[\left(q-p_{1}-p_{2}\right)^{2}-M_{N}^{2}\right]} .
$$

Setting $\mathrm{n}=4$ and neglecting all masses and momenta except $M_{N}$, we obtain

$$
\mathcal{M}_{N N \phi} \sim M_{N}^{4} \int \frac{d^{4} q}{\left(q^{2}-M_{N}^{2}\right) q^{2}\left(q^{2}-M_{N}^{2}\right)}
$$

The integral has to be of the form $\left(M_{N}\right)^{k}$; the power counting yields $k=-2$, so

$$
\mathcal{M}_{N N \phi} \sim M_{N}^{4} M_{N}^{-2}=M_{N}^{2}
$$

The exact result is (see Eqs. 5.2, 5.14)

$$
\mathcal{M}_{N N \phi}=+\frac{1}{8 s_{W}^{3} c_{W}} \frac{M_{N}^{2}}{M_{W}^{2}}+\ldots
$$


As mentioned in Sec. 5.1, the contribution of $\mathcal{M}_{\phi \phi N}$ cancels with that of $\mathcal{M}_{\phi N}$, leaving $\mathcal{M}_{N N \phi}$ as the only amplitude with the nondecoupling behaviour. We will now shed more light on this curious cancellation. The way to go is to replace in the diagrams corresponding to these amplitudes the $\mathrm{Z}$ boson with the photon and to use a Ward-identity [34], which relates the vertex formfactors $F_{V, A}^{\gamma}$ evaluated at $\left(p_{1}+p_{2}\right)^{2}=0$ to electron self-energies represented by the counterterms $\delta Z_{V, A}$ :

$$
F_{V, A}^{\gamma}(0)+\delta Z_{V, A}=\frac{1}{4 s_{W} c_{W}} \frac{\Sigma_{\gamma Z}(0)}{M_{Z}^{2}}
$$

where $\Sigma_{\gamma Z}(0)$ is the term originating in the bosonic loops of the $\gamma-Z$ mixing. At zero $M_{N}$ the graphs with unphysical Higgs $\phi$ are negligible, however, with $M_{N}$ rising the two graphs dominate the left-hand side of Eq. 6.49: the vertex $\mathcal{M}_{\phi \phi N}^{\gamma}$ and the selfenergy $\mathcal{M}_{\phi N}^{\gamma}$. Since the right-hand side of Eq. 6.49 is not affected by the NHL's, it remains constant and (very) small with respect to $\mathcal{M}_{\phi \phi N}^{\gamma}$ or $\mathcal{M}_{\phi N}^{\gamma}$ at $M_{N}=O(\mathrm{TeV})$. Hence the only way to meet the above formula is to have $\mathcal{M}_{\phi N}^{\gamma}=-\mathcal{M}_{\phi \phi N}^{\gamma}$ in the limit of large $M_{N}$.

If we now return from the photon to the $\mathrm{Z}$ boson, it suffices to check how the Feynman rules for the vertices change. It turns out that

$$
\begin{gathered}
\mathcal{M}_{\phi N} \equiv \mathcal{M}_{\phi N}^{Z}=c \mathcal{M}_{\phi N}^{\gamma} \\
\mathcal{M}_{\phi \phi N} \equiv \mathcal{M}_{\phi \phi N}^{Z}=c \mathcal{M}_{\phi \phi N}^{\gamma}
\end{gathered}
$$

with the same constant c (a simple function of Weinberg angle) in both eqs., so we conclude that

$$
\mathcal{M}_{\phi N}=-\mathcal{M}_{\phi \phi N}
$$

Note that for the photon case the form factors $F_{V, A}^{\gamma}$ are evaluated at $\left(p_{1}+p_{2}\right)^{2}=0$, while for the $\mathrm{Z}$ case $F_{V, A}$ are calculated at $\left(p_{1}+p_{2}\right)^{2}=M_{Z}^{2}$; this is acceptable, since 
in the limit of a large $M_{N}$ the $\mathrm{Z}$ mass can be neglected.

This cancellation implies that the formfactors are dominated by the single amplitude $\mathcal{M}_{N N \phi}$ :

$$
\hat{F}_{V}=\hat{F}_{A}=\frac{\alpha}{4 \pi} l_{2} \frac{1}{8 s_{W}^{3} c_{W}} \mathcal{X}+\ldots
$$

As in the case of the oblique parameter $\hat{\Pi}_{Z}$, we have to carefully examine the reliability of this approximation. We note again the leading term is suppressed by the mixing parameter $l_{2}=\tau \tau_{m i x}^{2}$ l. On the other hand, some of the higher order terms (found in many of the irreducible vertex diagrams, not just $\mathcal{M}_{N N \phi}$ ) are only linear in $\tau \tau_{m i x}$, which implies they can be larger than expected on the basis of an $M_{N}$ expansion. In Table 6.2 we show numerical predictions for the (exact) formfactor $\hat{F}_{V}$ and compare them with the approximate parameter $\hat{F}_{\text {Vappx }}=\hat{F}_{V}^{S M}+\hat{F}_{\text {Vappx }}^{N H L}$ where $\hat{F}_{\text {Vappx }}^{N H L}$ is the leading term in Eq. 6.53. The dependence of $\hat{F}_{V}^{S M}$ on the NHL mass has its origin in a different value of the input parameter $M_{W}$ (as calculated from $G_{\mu}$, see Sec. 6.5) for different NHL masses. Input numbers used are $M_{Z}=91.1884 \mathrm{GeV}$, $M_{H}=200 \mathrm{GeV}, m_{t}=176 \mathrm{GeV}, \tau \tau_{\text {mix }}=0.033$ and $e e_{\text {mix }}=\mu \mu_{\text {mix }}=0$.

\begin{tabular}{|l|r|r|r|r|l|}
\hline$M_{N}$ & $0.5 \mathrm{TeV}$ & $1 \mathrm{TeV}$ & $3 \mathrm{TeV}$ & $5 \mathrm{TeV}$ & \\
\hline$\hat{F}_{V}^{S M}$ & 1.938 & 1.939 & 1.949 & 1.971 & $\times 10^{-3}$ \\
$\hat{F}_{V}$ & 2.056 & 2.247 & 3.525 & 5.903 & $\times 10^{-3}$ \\
$\hat{F}_{\text {Vappx }}=\hat{F}_{V}^{S M}+\hat{F}_{\text {Vappx }}^{N H L}$ & 1.971 & 2.071 & 3.150 & 5.345 & $\times 10^{-3}$ \\
$\hat{F}_{\text {Vappx }}^{\text {NHL }}$ & 0.033 & 0.132 & 1.201 & 3.374 & $\times 10^{-3}$ \\
$d=\hat{F}_{V}-\hat{F}_{\text {Vappx }}$ & 0.085 & 0.176 & 0.375 & 0.558 & $\times 10^{-3}$ \\
\hline
\end{tabular}

Table 6.2: Comparison of $\hat{F}_{V a p p x}$ with $\hat{F}_{V}$

For $M_{N}=0.5 \mathrm{TeV}, \hat{F}_{V a p p x}^{N H L}$ contributes $0.033 \times 10^{-3}$ of the total difference between $\hat{F}_{V}^{S M}$ and $\hat{F}_{V}$. It is less than the contribution of the higher order terms,

\footnotetext{
${ }^{2}$ Assuming $e e_{\text {mix }}=\mu \mu_{\text {mix }}=0$ and $Z \rightarrow \tau \tau$ mode
} 
$d=0.085 \times 10^{-3}$. However, at $3 \mathrm{TeV}$ the roles are switched, with $\hat{F}_{\text {Vappx }}^{N H L}$ dominating as expected.

Overall, $\hat{F}_{\text {Vappx }}$ differs from $\hat{F}_{V}$ by approximately $4 \%$ at $0.5 \mathrm{TeV}$ and by $9.5 \%$ at $5 \mathrm{TeV}$. This is worse than the $1 \%$ found for the $\hat{\Pi}_{Z}$ parameter and it signals that the terms linear in mixing are more important in this case. Indeed, the second largest non-SM contribution to $\hat{F}_{V}$ comes from the graph of Fig. 6.7d (associated with amplitude $\mathcal{M}_{W W N}$ ). It is given by (see Eqs. 5.13, 5.14)

$$
-\frac{\alpha}{4 \pi} l_{1} \frac{3 c_{W}}{4 s_{W}^{3}}\left(\frac{5}{6}-\ln M_{N}^{2}\right)
$$

and even at $5 \mathrm{TeV}$ it is as large as $2.02 \times 10^{-3}$ compared to $\hat{F}_{\text {Vappx }}^{N H L}=3.374 \times 10^{-3}$. Other terms are also relatively large, partly cancelling the $\mathcal{M}_{W W N}$ effect to produce the comparatively small difference of $9.5 \%$.

\subsection{Imprecise $M_{W}$, precise $G_{\mu}$}

Our on-shell renormalization scheme takes $\alpha, M_{Z}$ and $M_{W}$ as input parameters (see Sec. 4.3). However, the direct measurement of $M_{W}$ [53],

$$
M_{W}=80.410 \pm 0.180 \mathrm{GeV},
$$

as opposed to that of $M_{Z}$ [52],

$$
M_{Z}=91.1884 \pm 0.0022 \mathrm{GeV}
$$

is not yet precise enough for its use as an input parameter. To appreciate this point, let us examine the sensitivity of $\Gamma_{l l}$ to $M_{W}$. It mainly comes from the tree-level 
formula (loops are suppressed by factors of $\alpha$ )

$$
\Gamma_{0}=\frac{\alpha}{3} M_{Z}\left(v_{l}^{2}+a_{l}^{2}\right)=\frac{\alpha}{3} M_{Z} \frac{1-4 s_{W}^{2}+8 s_{W}^{4}}{8 s_{W}^{2} c_{W}^{2}}
$$

where $c_{W}^{2}=M_{W}^{2} / M_{Z}^{2}$. It turns out that $\sigma_{M_{W}}=0.18 \mathrm{GeV}$ induces $\sigma_{\Gamma_{0}} \doteq \sigma_{\Gamma_{l l}}$ $\doteq 1 \mathrm{MeV}$. This is very large compared to the experimental value (see Eq. 6.4)

$$
\Gamma_{l l}^{e x p}=83.93 \pm 0.14 \mathrm{MeV}
$$

As a consequence $M_{W}$, even though convenient as an input parameter for one-loop calculations, is usually replaced by the more precisely measured muon decay constant $G_{\mu}$ [2] :

$$
G_{\mu}=1.16637(2) \times 10^{-5} \mathrm{GeV}^{-2}
$$

The replacement is done in such a way that while $M_{W}$ is still kept in our formulae for one-loop self-energies and vertices, its actual value is no longer taken from the direct measurement, but is rather calculated from $G_{\mu}$. To calculate $M_{W}$ from $G_{\mu}$, one first computes the $\mu$-decay rate to one-loop level in the Fermi model (this defines $G_{\mu}$ ) and then equates it to the one-loop calculation of the same quantity in the SM [34]. The result is a formula relating $M_{W}$ to $G_{\mu}$ :

$$
M_{W}^{2} s_{W}^{2}=\frac{\pi \alpha}{\sqrt{2} G_{\mu}\left(1-\Delta r^{S M}\right)}
$$

with $\Delta r^{S M}$ (the notation $\Delta r$ is reserved for our model) representing loop effects in $\mu$-decay,

$$
\Delta r^{S M}=\Delta r^{S M}\left(\alpha, M_{W}, M_{Z}, M_{H}, m_{t}\right)
$$




$$
\begin{aligned}
& =\frac{\mathcal{R} e \hat{\Sigma}_{W}^{S M}(0)}{M_{W}^{2}}+\frac{\alpha}{4 \pi s_{W}^{2}}\left(6+\frac{7-4 s_{W}^{2}}{2 s_{W}^{2}} \ln c_{W}^{2}\right) \\
& =\frac{\mathcal{R} e \hat{\Sigma}_{W}^{S M}(0)}{M_{W}^{2}}+\delta_{V}^{S M} .
\end{aligned}
$$

$M_{W}$ is found from Eq. 6.59 by iterative procedure.

The reason we discuss this in some detail is that our model modifies this relation between $G_{\mu}$ and $M_{W}$ both at tree and one-loop level and thus NHL's, besides contributing directly to $\Gamma_{l l}$ through Z-decay loops, contribute also indirectly via $M_{W}$. This complication in the calculation of $\Gamma_{l l}$ is something we can actually benefit from since in $M_{W}$ we obtained another observable sensitive to NHL's. In our model, Eq. 6.59 is modified as

$$
M_{W}^{2} s_{W}^{2}=\frac{\pi \alpha}{\sqrt{2} G_{\mu}(1-\Delta r)}\left(1-\frac{1}{2} e e_{m i x}-\frac{1}{2} \mu \mu_{m i x}\right),
$$

where $1-\frac{1}{2} e e_{m i x}-\frac{1}{2} \mu \mu_{m i x}$ represents the tree-level modification 3 . The loop quantity $\Delta r$ is calculated from the diagrams depicted in Figs. 7.1-7.4 and the diagrams with the corrected W propagator. We devote the next chapter to the detailed calculation of the $\Delta r$ for the arbitrary values of the mixings $l l_{\text {mix }}$. Here we make a specific choice of mixings which will help us to avoid most of the $\mu$-decay loop diagrams: we put $e e_{m i x}=\mu \mu_{m i x}=0$. This choice also implies $e \mu_{m i x}=e \tau_{m i x}=\mu \tau_{m i x}=0$ and leaves $\tau \tau_{\text {mix }}$ as the only nonzero mixing parameter. Of the non-SM $\mu$-decay loops, the vertex corrections, neutrino self-energy corrections and the boxes are all proportional to either $e e_{m i x}$ or $\mu \mu_{m i x}$ and therefore only $\hat{\Sigma}_{W}$ (see Sec. 6.3), the $W$ propagator correction remains to modify $\Delta r^{S M}$ :

$$
\Delta r=\frac{\mathcal{R} e \hat{\Sigma}_{W}(0)}{M_{W}^{2}}+\delta_{V}=\frac{\mathcal{R} e \hat{\Sigma}_{W}(0)}{M_{W}^{2}}+\delta_{V}^{S M} .
$$

\footnotetext{
${ }^{3} \Gamma_{\mu}$ is modified by $1-e e_{m i x}-\mu \mu_{m i x}$ and we take the square root of $\Gamma_{\mu}$ to get Eq. 6.61.
} 
In the limit of $M_{N} \gg M_{W}$ we obtain an expression similar to Eq. 6.30,

$$
\Delta r=\Delta r^{S M}-\frac{\alpha}{\pi}\left\{\frac{c_{W}^{2}}{16 s_{W}^{4}} k_{H H} \frac{M_{N}^{2}}{M_{W}^{2}}+O\left(\ln M_{N}^{2} / M_{W}^{2}\right)+\ldots\right\}
$$

The neglect of terms beyond the $O\left(M_{N}^{2} / M_{W}^{2}\right)$ results in an error less than $2.5 \%$ for $M_{N}<5 \mathrm{TeV}$.

\subsection{Violation of the decoupling theorem}

The reader may wonder whether NHL loop effects can have any noticeable effect at all. After all, these loops are suppressed by factors such as $l l_{\text {mix }}$ or even $l l_{\text {mix }}^{2}$ (see for example Eq. 6.63, where $k_{H H}=\tau \tau_{\text {mix }}^{2}$ ) compared to their SM counterparts. Although this reasoning is correct, it is not complete. In spite of the smallness of the mixing parameters, the NHL loop effects can actually be larger than SM ones. The reason is the (possibly) large NHL mass and a violation of the decoupling theorem.

The decoupling theorem was established and proven by Appelquist and Carazzone in Ref. [59]. It describes how the heavy particles of a renormalizable theory $A$ enter into the low-energy theory $B$ [60]: All effects of the heavy particle in the low-energy theory $B$ appear either as a renormalization of the coupling constants or else are suppressed by powers of the heavy particle mass. For instance, heavy $W$ and $Z$ bosons of the SM (theory $A$ ) decouple from the low-energy QED (theory $B$ ).

There are, however, cases in spontaneously broken theories when the decoupling theorem is violated. A well-known example can be found in the SM itself with respect to top quark behaviour. There are two diagrams (Fig. 6.11) which exhibit a quadratic dependence on the top quark mass and therefore do not vanish as $m_{t} \rightarrow \infty$.

The nondecoupling effects are easily visible in this case - the diagrams of Fig. 6.11 led to indirect bounds on the top quark mass from LEP I observables. The bounds, $m_{t}=170 \pm 10_{-19}^{+17} \mathrm{GeV}$ [52], are actually competitive with the value obtained so far 

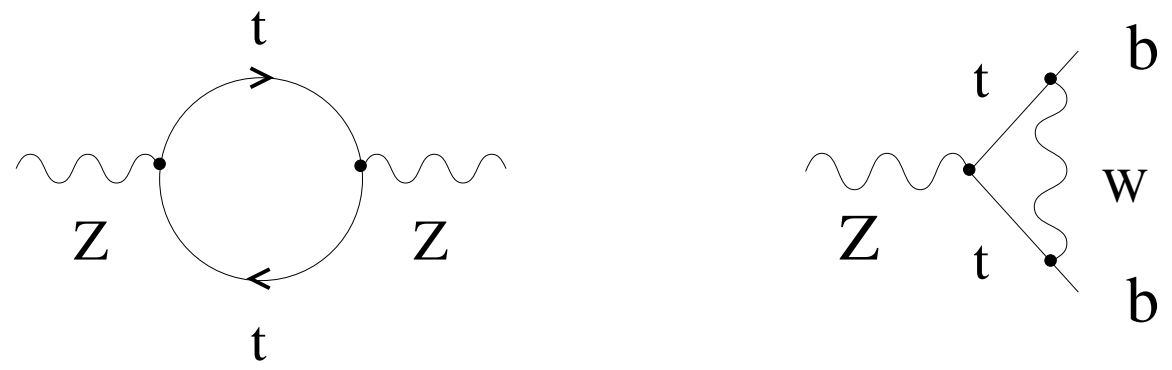

Figure 6.11: Diagrams with the top quark nondecoupling.

from the direct observation at $\mathrm{CDF}$ at Fermilab, $m_{t}=176 \pm 8 \pm 10 \mathrm{GeV}$ 61.

The NHL's also exhibit nondecoupling as can be seen in Eqs. 6.30, 6.33, 6.63, where the dominant terms are quadratically dependent on $M_{N}$. We note however, that the nondecoupling of NHL's is the consequence of our treatment of the mixings as the parameters independent of $M_{N}$. If we replaced $\tau \tau_{m i x}$ with $\tau \tau_{m i x} \sim \frac{D^{2}}{M_{N}^{2}}$ $\left(K_{H} \sim \frac{D}{M_{N}}\right)$, the dominant terms would change from $\sim \tau \tau_{m i x}^{2} M_{N}^{2}$ (see Eqs. 6.30, $6.33,6.63)$ to

$$
\tau \tau_{m i x}^{2} M_{N}^{2} \rightarrow \frac{D^{4}}{M_{N}^{4}} M_{N}^{2}=\frac{D^{4}}{M_{N}^{2}}
$$

and the decoupling would be recovered.

The analogy with the top quark can be useful in another aspect. We can make a naive estimate of how large an NHL mass $M_{N}$ should be to produce an effect comparable to that of the top quark. For example, oblique corrections rise with the top quark mass as $\hat{\Pi}_{Z}^{S M} \sim m_{t}^{2} / M_{W}^{2}$, 团 while in our model, the dependence on $M_{N}$

\footnotetext{
${ }^{4}$ According to Okun [62], this point is subtle: a large positive contribution from the top quark is cancelled by a large negative contribution from all other virtual particles. As a result, genuine electroweak corrections are negligible and the bulk of $\sim 2.5 \mathrm{MeV}$ loop corrections is associated with the running coupling constant $\alpha$.
} 
(besides the dependence on $m_{t}$ ) is

$$
\hat{\Pi}_{Z} \sim k_{H H} \frac{M_{N}^{2}}{M_{W}^{2}} \doteq \tau \tau_{m i x}^{2} \frac{M_{N}^{2}}{M_{W}^{2}}
$$

yielding

$$
m_{t}^{2} \sim \tau \tau_{m i x}^{2} M_{N}^{2}
$$

As a result, for $\tau \tau_{\text {mix }}=0.033, M_{N} \doteq 30 m_{t} \doteq 5 \mathrm{TeV}$ is required to produce 'the top size' effect. We note this is not the necessary condition for observing the NHL given the current mixings. As our numerical results indicate, even NHL's with the mass $M_{N} \sim 3 \mathrm{TeV}$ could lead to non-SM effects in $\Gamma_{l l}$. In the same sense we do not have to change the top mass by $175 \mathrm{GeV}$ to see conflict with the data.

\subsection{Heavy NHL's and perturbation theory break- down}

With one-loop corrections rising as $M_{N}^{2} / M_{W}^{2}$, we have to know at what value of $M_{N}$ these corrections are comparable in size to the tree-level contribution. At this point the theory becomes strongly interacting and the perturbative treatment fails. Alternatively, we can study the transition to the strongly interacting regime through the tree-level width of the NHL. We investigate this latter approach here and show the analogy to the the strongly interacting Higgs.

There are three decay modes of an NHL, $N_{a} \rightarrow W^{ \pm}+l^{\mp}, N_{a} \rightarrow Z+\nu_{i}$ and $N_{a} \rightarrow H+\nu_{i}$, open for $M_{N}>M_{W}, M_{Z}, M_{H}$. They are represented by the diagrams of Fig. 6.12. In the limit $M_{N} \gg M_{W}, M_{Z}, M_{H}$, the partial decay widths are given 

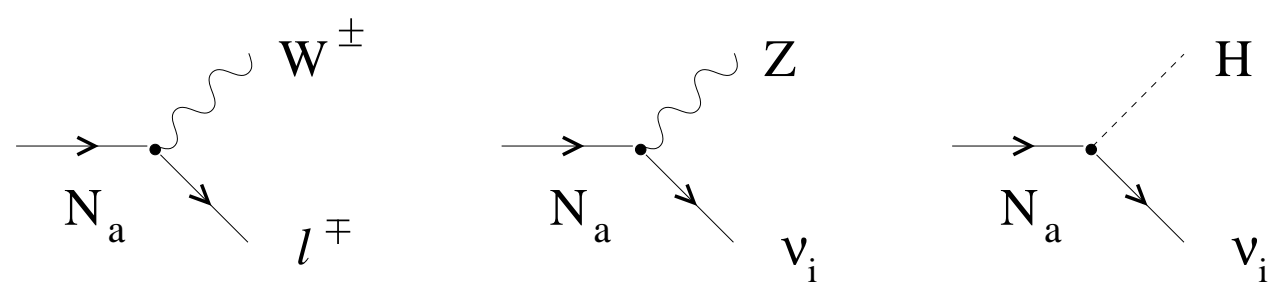

Figure 6.12: Decay modes of an NHL.

by

$$
\begin{aligned}
\sum_{l=e, \mu, \tau} \Gamma\left(N_{a} \rightarrow W^{ \pm}+l^{\mp}\right) & =\frac{\alpha}{16 s_{W}^{2}} a_{\text {mix }} \frac{M_{N}^{3}}{M_{W}^{2}}, \\
\sum_{i=1,2,3} \Gamma\left(N_{a} \rightarrow H+\nu_{i}\right) & =\frac{\alpha}{32 s_{W}^{2}} a_{\text {mix }} \frac{M_{N}^{3}}{M_{W}^{2}}, \\
\sum_{i=1,2,3} \Gamma\left(N_{a} \rightarrow Z+\nu_{i}\right) & =\frac{\alpha}{32 s_{W}^{2}} a_{\text {mix }} \frac{M_{N}^{3}}{M_{W}^{2}},
\end{aligned}
$$

where the following relations were used:

$$
\sum_{i}\left|\left(K_{L}^{\dagger} K_{H}\right)_{i a}\right|^{2} \doteq \sum_{l}\left|\left(K_{H}\right)_{l a}\right|^{2}=a_{m i x}
$$

The total tree-level width of the NHL is the sum of the partial widths:

$$
\Gamma_{N}=\frac{3 \alpha}{16 s_{W}^{2}} a_{m i x} \frac{M_{N}^{3}}{M_{W}^{2}}
$$

\footnotetext{
${ }^{5}$ The partial width for $N_{a} \rightarrow W^{ \pm}+l^{\mp}$ is the same as in the see-saw model of Ref. 19, while the widths for $N_{a} \rightarrow Z+\nu_{i}$ and $N_{a} \rightarrow H+\nu_{i}$ are half of their see-saw counterparts.
} 
For a comparison, the tree-level width of a very heavy Higgs is given by

$$
\Gamma_{H}=\frac{3 \alpha}{32 s_{W}^{2}} \frac{M_{H}^{3}}{M_{W}^{2}} .
$$

Both $\Gamma_{N}$ and $\Gamma_{H}$ rise swiftly with the particle mass and at some critical point they become larger than the masses $M_{N}, M_{H}$ themselves - a clear indication of a perturbative breakdown. The tree-level formulae are no longer appropriate. We can get a safe estimate on the critical mass by demanding that

$$
\Gamma_{N, H} \leq \frac{1}{2} M_{N, H}
$$

With $a_{m i x} \leq e e_{m i x}+\mu \mu_{m i x}+\tau \tau_{m i x} \doteq \tau \tau_{m i x}=0.033$, we obtain

$$
M_{N}^{\text {crit }} \sim 4 \mathrm{TeV}
$$

and for the Higgs we get the well-known bound of $M_{H}^{\text {crit }} \sim 1 \mathrm{TeV}$. Note that $M_{N}^{\text {crit }}$ is not to be interpreted as the upper bound on the NHL mass. Its sole purpose is to make us aware of the limitations of the perturbative treatment.

\subsection{Results}

In this section we present our numerical results.

Our FORTRAN program is written as a modification of the routines from the CERN electroweak library [57] used for the SM predictions of LEP I parameters. This applies namely to the oblique parameter, $\hat{\Pi}_{Z}$, with most of the contributing diagrams (see Fig. 6.2 -6.5) being SM. The non-SM contributions were implemented as specified in Sec. 6.3. The vertex factor $\delta \hat{\Gamma}_{l l}$ was derived independently of the

\footnotetext{
${ }^{6} \mathrm{~A}$ similar value is obtained from considerations of perturbative unitarity violation.
} 
CERN electroweak library, including the SM diagrams.

As a 'standard set' of input parameters, we used $M_{Z}=91.1884 \mathrm{GeV}, \alpha^{-1}=$ 137.036, $A \equiv \frac{\pi \alpha}{\sqrt{2} G_{\mu}}=37.281 \mathrm{GeV}$. Also part of this set are $M_{H}=200 \mathrm{GeV}$ and $m_{t}=176 \mathrm{GeV}$. Below we only make it explicit when values different from the standard set are used, e.g., $M_{H}, m_{t}$ in Figs. 6.13, 6.17a. To keep the discussion simple (without losing any qualitative features), we have reduced the free parameter space by assuming degenerate masses for the three NHL's. In this chapter, we have also imposed restrictions on the mixing parameters. We assume that $e e_{m i x}$ and $\mu \mu_{\text {mix }}$ are very small relative to $\tau \tau_{\text {mix }}$. The model and NHL mass independent limits quoted in Eq. 3.38 are more stringent for $e$ and $\mu$ than for $\tau$. In addition, our assumption is also partially 1 supported by the smallness of $e \mu_{m i x}$ (see Eq. 3.44), as determined from $\mu \rightarrow e \gamma$, in combination with the inequality Eq. 3.27. This neglect of $e e_{m i x}$ and $\mu \mu_{m i x}$ proves useful practically in that many of the muon decay loops (boxes and vertex corrections, but not $W$ oblique correction) are eliminated as a result. The general case of arbitrary $e e_{m i x}, \mu \mu_{m i x}$ is considered in the next chapter. We present results mainly for the NHL mass range $0.5 \mathrm{TeV} \leq M_{N} \leq 5 \mathrm{TeV}$, as motivated by the non-decoupling arguments given in Sec. 6.6.

Given the relative complexity of the formulae involved, we looked for alternative ways of checking them, other than the special care taken in their derivation.

First, a logical step to take is to run our program with all mixing parameters zero, to see if it reduces to the SM, as predicted by the CERN electroweak library. For the standard set of input parameters we get precise agreement, see Table 6.3 .

Second, we checked that infinities cancelled out in all renormalized quantities.

Third, throughout this chapter we tried to separate the dominating contributions in the limit of large NHL mass and represent them by simple formulae. Here, we collect these approximations and see, if we can understand the behaviour of partial

\footnotetext{
${ }^{7}$ Partially, because small $e \mu_{m i x}$ does not necessarily imply small $e e_{m i x}$ and $\mu \mu_{m i x}$.
} 


\begin{tabular}{|l|c|c|c|}
\hline & $\hat{\Pi}_{Z}^{S M}$ & $\delta \hat{\Gamma}_{l l}^{S M}$ & $\Gamma_{l l}^{S M}[\mathrm{MeV}]$ \\
\hline CERN library & $-4.29769 \times 10^{-2}$ & $-1.16976 \times 10^{-3}$ & 84.0297 \\
Our program & $-4.29769 \times 10^{-2}$ & $-1.16976 \times 10^{-3}$ & 84.0297 \\
\hline
\end{tabular}

Table 6.3: SM limit of our model

leptonic widths on their basis. Thus, collecting Eqs. 6.2, 6.30, 6.33 and 6.53, we get

$$
\begin{aligned}
\Gamma_{l l} & =\frac{\Gamma_{0}+\delta \hat{\Gamma}_{l l}}{1+\hat{\Pi}_{Z}\left(M_{Z}^{2}\right)}\left(1+\delta_{Q E D}\right) \doteq \Gamma_{l l}^{a p p x} \\
& =\frac{\Gamma_{0}+\delta \hat{\Gamma}_{Z}^{S M}+\frac{2}{3} \alpha M_{Z}\left(v_{l} \hat{F}_{\text {Vappx }}^{N H L}+a_{l} \hat{F}_{\text {Vappx }}^{N H L}-v_{l} \hat{\Pi}_{\gamma Z \text { app }}^{N H L}\right)}{1+\hat{\Pi}_{Z}^{S M}+\hat{\Pi}_{Z \text { app }}^{N H L}} \\
& \times\left(1+\delta_{Q E D}\right),
\end{aligned}
$$

where

$$
\begin{aligned}
\hat{\Pi}_{Z a p p x}^{N H L} & =\frac{\alpha}{\pi} \frac{c_{W}^{2}-s_{W}^{2}}{16 s_{W}^{4}} \frac{M_{N}^{2}}{M_{W}^{2}} k_{H H}, \\
\hat{\Pi}_{\gamma Z \text { appx }}^{N H L} & =-\frac{\alpha}{\pi} \frac{c_{W}}{16 s_{W}^{3}} \frac{M_{N}^{2}}{M_{W}^{2}} k_{H H}, \\
\hat{F}_{\text {Vappx }}^{N H L} & =\frac{\alpha}{4 \pi} \frac{1}{8 s_{W}^{3} c_{W}} \frac{M_{N}^{2}}{M_{W}^{2}} l_{2} .
\end{aligned}
$$

The third, fourth and the fifth term in the numerator in $\Gamma_{l l}^{a p p x}$ are all increasingly negative with rising $M_{N}$, therefore they make the partial width smaller. The denominator rises with $M_{N}$ and also makes the partial width smaller. On the other hand, the tree-level result, $\Gamma_{0}$, whose indirect dependence on NHL's via $M_{W}$ was discussed in Sec. 6.5, rises with $M_{N}$ (see Table 6.4). As a result, depending on the particular values of the mixing parameters $l_{2}$ and $k_{H H}$, the partial width can either increase with NHL mass or decrease. An example of the latter case can be found in Table 6.4 which shows (for the standard set of input parameters, $\tau \tau_{\text {mix }}=0.033$ and the above assumptions on $\left.e e_{m i x}, \mu \mu_{m i x}\right)$ the $Z \rightarrow \tau \tau$ mode partial width $\Gamma_{\tau \tau}$ for 
different NHL masses. Also shown are $\Gamma_{0}$ and $\Gamma_{\tau \tau}^{a p p x}$, the prediction of Eq. 6.73.

\begin{tabular}{|l|r|r|r|r|}
\hline$M_{N}$ & $0.5 \mathrm{TeV}$ & $1 \mathrm{TeV}$ & $3 \mathrm{TeV}$ & $5 \mathrm{TeV}$ \\
\hline$\Gamma_{0}[\mathrm{MeV}]$ & 81.43 & 81.45 & 81.82 & 82.60 \\
$\Gamma_{\tau \tau}[\mathrm{MeV}]$ & 83.99 & 83.94 & 83.62 & 83.02 \\
$\Gamma_{\tau \tau}^{a p p x}[\mathrm{MeV}]$ & 84.00 & 83.97 & 83.70 & 83.18 \\
\hline
\end{tabular}

Table 6.4: $\Gamma_{0}$ and $\Gamma_{\tau \tau}$ as a function of $M_{N}$

Once we can predict partial leptonic widths, we can take advantage of that and study the universality breaking parameter defined as 49.

$$
U_{b r}=\left|\frac{\Gamma_{\tau \tau}-\Gamma_{e e}}{\Gamma_{\tau \tau}+\Gamma_{e e}}\right|=\left|\frac{\delta \hat{\Gamma}_{\tau \tau}-\delta \hat{\Gamma}_{e e}}{2 \Gamma_{0}+\delta \hat{\Gamma}_{\tau \tau}+\delta \hat{\Gamma}_{e e}}\right|
$$

The new feature here is that the universality breaking parameter depends only on the vertex factor $\delta \hat{\Gamma}_{l l}$; the indirect influence of $M_{W}$ via $\Gamma_{0}$ is suppressed and the direct oblique corrections cancell out in the ratio. Thus $U_{b r}$ represents an independent and complementary quantity to the partial leptonic widths for the study of the NHL's. The experimental limit on the universality breaking parameter can be derived from the limits on partial leptonic widths as follows. From the limits on partial leptonic widths (see Eq. 6.5) we get

$$
\frac{\Gamma_{\tau \tau}}{\Gamma_{e e}}=0.9991 \pm 0.0040
$$

This ratio implies

$$
U_{b r}^{e x p} \doteq\left|\frac{\Gamma_{\tau \tau}-\Gamma_{e e}}{\Gamma_{e e}+\Gamma_{e e}}\right|=\left|\frac{\Gamma_{\tau \tau}}{2 \Gamma_{e e}}-\frac{1}{2}\right|=0.00045 \pm 0.00200
$$

leading to the upper limit (at $2 \sigma$ level)

$$
U_{b r}^{e x p}<0.00445
$$


The third experimental parameter sensitive to NHL's is the W mass, $M_{W}$. Under the assumptions made above it is only sensitive to $W$ oblique corrections and thus complements the former two quantities.

The $Z$ leptonic widths are given as a function of NHL mass in Figs. 6.13 - 6.15. We mostly show $\Gamma_{\tau \tau}$, as the most NHL-sensitive mode under the stated assumptions on mixing parameters. $\Gamma_{e e}=\Gamma_{\mu \mu}$ is also plotted in Figs. 6.13a, 6.14b . The dashed lines represent the $1 \sigma$ variation about the current experimental results for the individual $Z$ leptonic widths $\Gamma_{\tau \tau}^{e x p}$ or $\Gamma_{e e}^{e x p}$, see Eq. 6.5.

In Fig. 6.13a, the widths $\Gamma_{e e}, \Gamma_{\tau \tau}$ are shown for three values of the top quark mass, $m_{t}=158,176$ and $194 \mathrm{GeV}$. The mixing was fixed at $\tau \tau_{m i x}=0.033$. The striking difference between the two modes can be seen to arise from the competition between the $\Gamma_{0}$ on one side and the $\delta \hat{\Gamma}_{Z}$ and $\hat{\Pi}_{Z}$ on the other side (see the discussion above). For the $\tau \tau$ mode, the mixing parameter $l_{2}=k_{H H}=\tau \tau_{m i x}^{2}$, while for the ee mode, $k_{H H}=\tau \tau_{m i x}^{2}$ and $l_{2}=0$ (see Eq. 6.39). Zero parameter $l_{2}$ eliminates the third and the fourth terms in the numerator in the second line of Eq. 6.73 and the rising $\Gamma_{0}$ dominates as a result. In Fig. $6.13 \mathrm{~b}$, we again set $\tau \tau_{m i x}=0.033$ and show the $Z$ width to $\tau^{+} \tau^{-}$for three values of the Higgs mass, $M_{H}=100,200,800 \mathrm{GeV}$. The dependence on the top quark mass and the weak dependence on the Higgs mass got transferred from the SM as expected.

In Fig. 6.14 we vary the mixings from $\tau \tau_{m i x}=0.02$ to $\tau \tau_{m i x}=0.07$. Fig. 6.14a shows $\Gamma_{\tau \tau}$, Fig. 6.14 $\mathrm{b}$ shows $\Gamma_{e e}$. The quadratic dependence on the mixing parameter, anticipated from Eq. 6.73, is confirmed.

Fig. 6.15 is the only figure where we look at a 'lower energy' scale. It is a continuation of Fig. 6.14a to the lower NHL mass range. As expected, the partial width is well within the $1 \sigma$ band and the NHL effects are negligible.

The universality breaking factor $U_{b r}$ is plotted in Fig. 6.16 as a function of $M_{N}$, with the mixing parameter varied about $\tau \tau_{m i x}=0.033$. The $1 \sigma$ experimental limit on $U_{b r}$ is indicated as the dashed line. 
Finally, we present the NHL mass dependence of the $W$ mass in Figs. 6.17a,b. The top quark mass is varied about $m_{t}=176 \mathrm{GeV}$ in Fig. 6.17a, while the mixing is held constant at $\tau \tau_{\text {mix }}=0.033$. In Fig. 6.17b, the mixing is varied about $\tau \tau_{m i x}=$ 0.033 for a fixed top quark mass.

\subsection{Discussion and Conclusions}

Our primary consideration here has been the inclusion of neutral heavy leptons in the calculation of the flavour-conserving $Z$ decays to charged leptons at one-loop level. The dependence of the $Z$ leptonic widths on the NHL mass, $M_{N}$, and on the mixing parameter $\tau \tau_{m i x}$ was given in Figs. 6.13-6.15. We see for the experimentally allowed upper limit of $\tau \tau_{m i x}=0.033$, and assuming $e e_{m i x}=\mu \mu_{m i x}=0, m_{t}=176 \mathrm{GeV}$, the $Z$ decay width to $\tau$ leptons becomes sensitive to NHL masses of about $4.3 \mathrm{TeV}$ at the $2 \sigma$ level. Curiously, this is an upper limit,

$$
M_{N} \leq 4.3 \mathrm{TeV}
$$

rather than the lower one. The cause is the nondecoupling of the NHL's.

Apart from this comparison of each leptonic width prediction with experiment we can also exploit the lepton flavour universality violation which takes place in the model. The universality breaking ratio, $U_{b r}$ (see Fig. 6.16), leads to a yet better upper limit,

$$
M_{N} \leq 3.8 \mathrm{TeV}
$$

at the $2 \sigma$ level. The importance of $U_{b r}$ is underlined by the fact that it is sensitive only to the vertex parameter $\delta \hat{\Gamma}_{l l}$, unlike $\Gamma_{l l}$, which besides the vertex parameter also depends on the $\mathrm{Z}$ oblique corrections and indirectly, via the $W$ boson mass, on the W oblique corrections. Thus the universality breaking complements the $\mathrm{Z}$ leptonic partial widths as far as sensitivity to NHL's is concerned. 


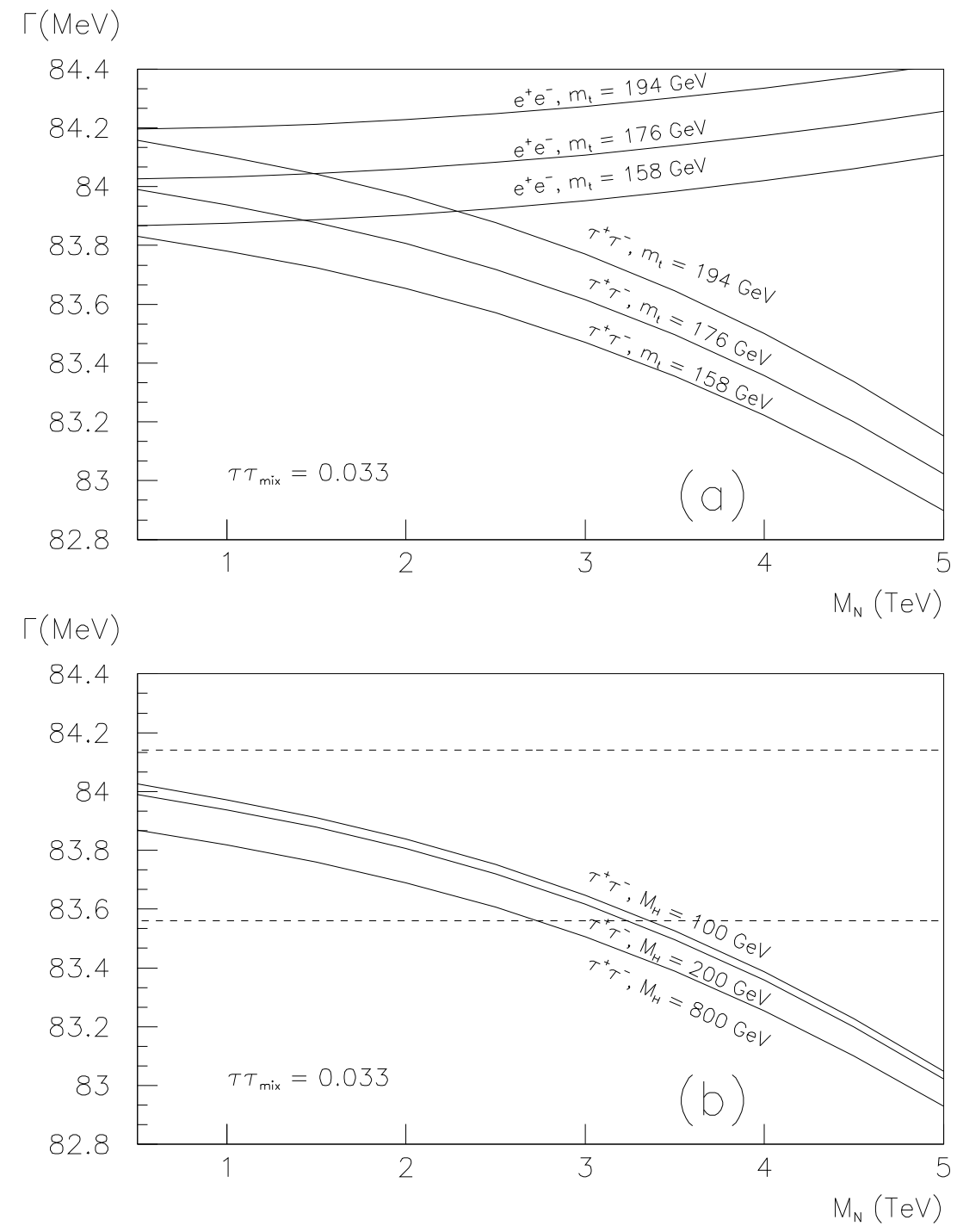

Figure 6.13: Z leptonic width as a function of $M_{N}$ in the interval $0.5 \mathrm{TeV} \leq M_{N} \leq$ $5 \mathrm{TeV}$ for (a) fixed mixing parameter $\tau \tau_{m i x}$, fixed Higgs mass and different values of $m_{t}$; both $Z \rightarrow \tau \tau$ and $Z \rightarrow e e$ modes shown, (b) fixed mixing parameter $\tau \tau_{m i x}$, fixed $m_{t}$ and different values of the Higgs mass $M_{H}$; only $Z \rightarrow \tau \tau$ mode shown. The dashed lines represent $1 \sigma$ band about the current experimental value $\Gamma_{\tau \tau}^{\exp }=$ $83.85 \pm 0.29 \mathrm{MeV}$. 

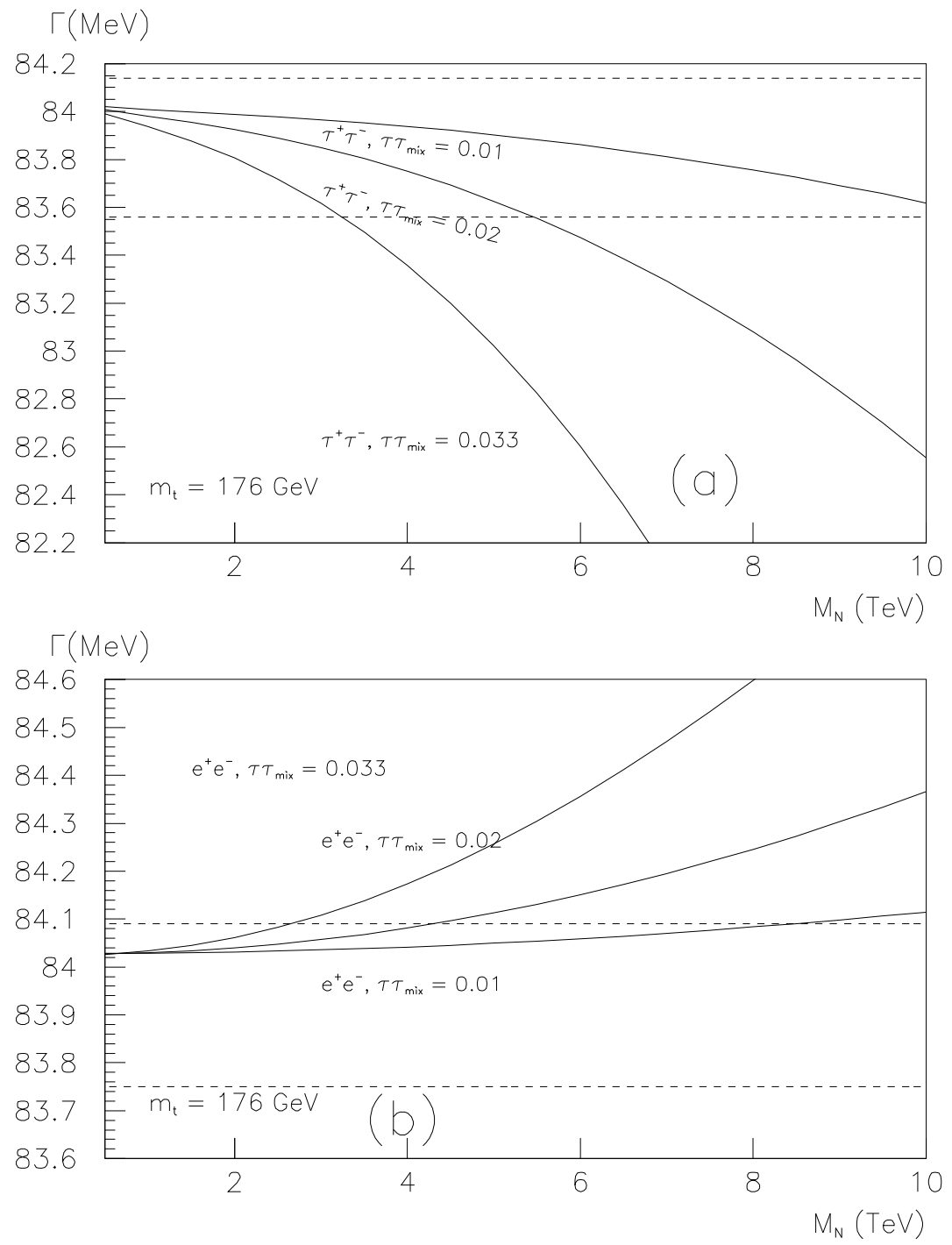

Figure 6.14: Z leptonic width as a function of $M_{N}$ in the interval $0.5 \mathrm{TeV} \leq M_{N} \leq$ $5 \mathrm{TeV}$ for fixed $m_{t}$, fixed Higgs mass and different values of the mixing parameter, (a) $Z \rightarrow \tau \tau$ mode, (b) $Z \rightarrow e e$ mode. The dashed lines represent $1 \sigma$ band about the current experimental value (a) $\Gamma_{\tau \tau}^{\exp }=83.85 \pm 0.29 \mathrm{MeV}$, (b) $\Gamma_{e e}^{\exp }=83.92 \pm 0.17 \mathrm{MeV}$. 


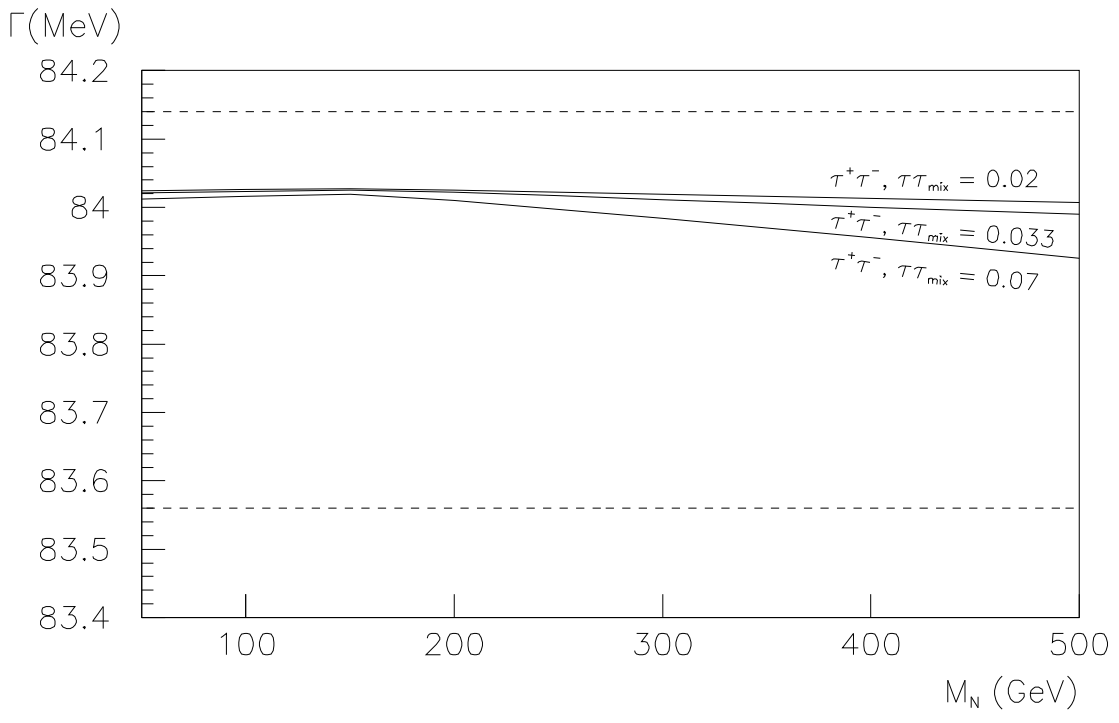

Figure 6.15: $Z \rightarrow \tau \tau$ width as a function of $M_{N}$ in the interval $50 \mathrm{GeV} \leq M_{N} \leq$ $500 \mathrm{GeV}$ for a fixed top mass $m_{t}=176 \mathrm{GeV}$ and different values of the mixing parameter $\tau \tau_{m i x}$. The dashed lines represent the $1 \sigma$ band about the current experimental value $\Gamma_{\tau \tau}^{\exp }=83.85 \pm 0.29 \mathrm{MeV}$. 


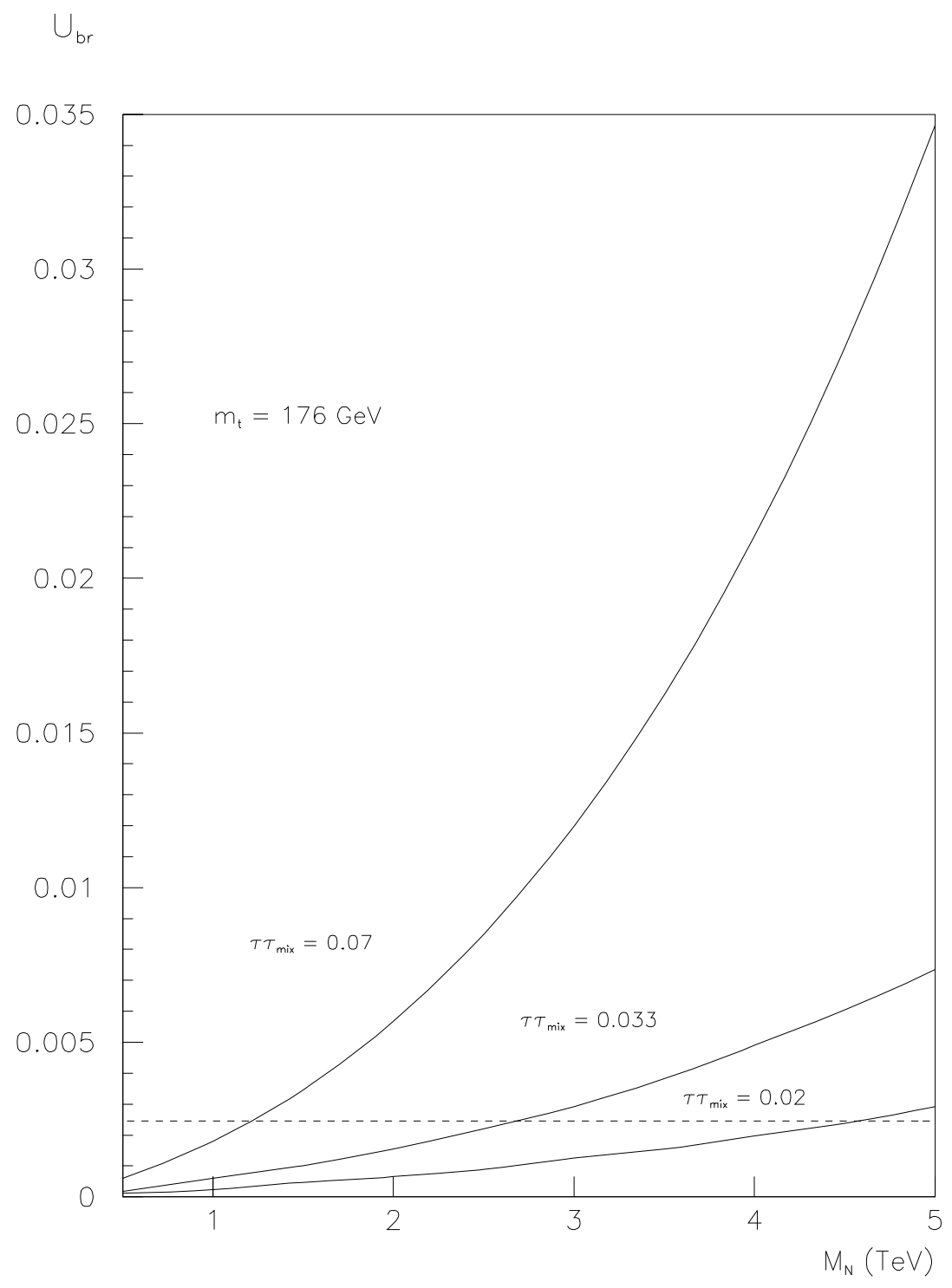

Figure 6.16: Universality breaking parameter $U_{b r}$ as a function of $M_{N}$ for fixed top quark mass $\left(m_{t}=176 \mathrm{GeV}\right)$ and different values of the mixing parameter. The dashed line represents $1 \sigma$ experimental limit $(<0.00245)$. 

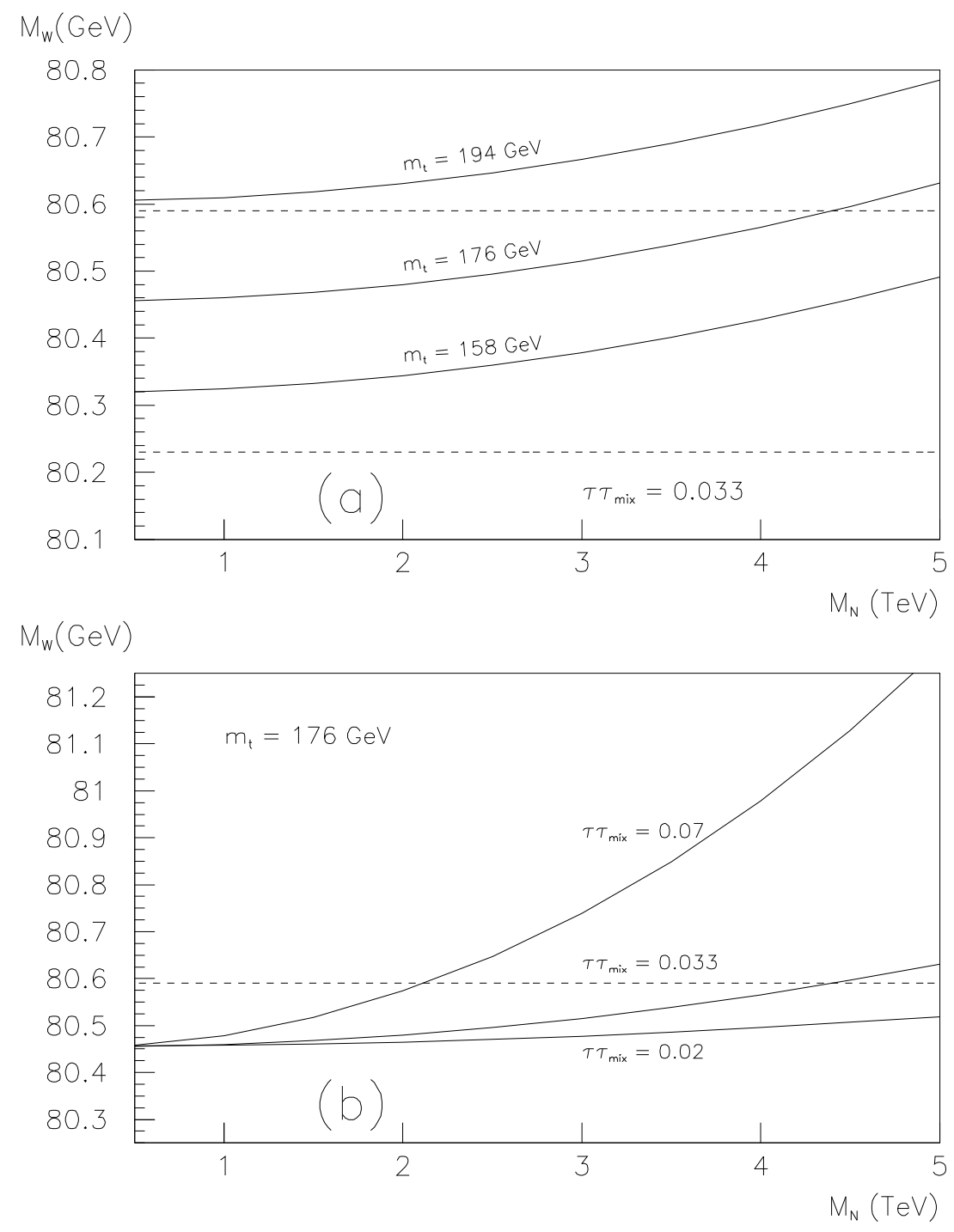

Figure 6.17: W mass as a function of $M_{N}$ for (a) fixed mixing parameter $\left(\tau \tau_{\text {mix }}=\right.$ 0.033 ) and different values of $m_{t}$, (b) fixed top quark mass $\left(m_{t}=176 \mathrm{GeV}\right)$ and different values of the mixing parameter. The dashed lines represent $1 \sigma$ band about the current experimental value $M_{W}=80.410 \pm 0.180 \mathrm{GeV}$. 
The $W$ boson mass also exhibits some sensitivity to NHL parameters arising from the mixing factor modifications and the presence of one-loop diagrams containing NHL's, as described in Sec. 6.5. From Figs. 6.17a,b we see that the sensitivity of the $W$ mass, currently measured as $M_{W}=80.410 \pm 0.180 \mathrm{GeV}$ [53, to NHL's depends to a large degree on the top mass. The experimental error on $M_{W}$ might be expected to come down to about $0.05 \mathrm{GeV}$ once LEP II measures $W$ pair production 63.

In conclusion to this chapter, the $\mathrm{Z}$ boson flavour-conserving leptonic widths along with the lepton universality breaking parameter and the $\mathrm{W}$ mass represent some of the best quantities sensitive to the NHL mass. This applies especially to NHL's that are far too heavy to be produced directly at present colliders. The only way to probe their mass in this case is via their loop contributions. Much of the previous effort on NHL studies has been so far concentrated on flavour-violating processes, either at very low energies ( $\mu, \tau$ decays, see Eq. 3.42), or at $Z$-peak energy ( $Z$ decays, see Eq. 3.43 and Sec. 5.1). We feel there are at least two reasons which give the processes studied in this thesis a distinct advantage over the flavour-violating decays.

First, we were able to actually reduce the allowed region in the mixings - NHL mass parameter space here (see Eqs. 6.79, 6.80) using the current experimental data. The only flavour-violating process that competes with the limits of Eqs. 6.79, 6.80 is the decay $\mu \rightarrow$ eee, which sets an upper limit on NHL mass (see Eqs. 5.29, 5.30, assuming $e e_{\text {mix }}=0.0071,\left|e \mu_{m i x}\right|=0.00024$ ) at $2 \sigma$ level

$$
M_{N} \leq 3 \mathrm{TeV}
$$

The flavour-violating decay rates for $\tau$ and $Z$ are below the current experimental sensitivity (see Secs. 5.1.3, 5.2).

Second, the inequality Eq. 3.27 can further suppress the flavour-violating processes against the flavour-conserving ones via the 'conspiracy of the phases' in the 
sum of complex terms making up the flavour-violating parameters $l l_{m i x}^{\prime}$. In this case, flavour-conserving processes are the only way to probe very heavy NHL's. 


\section{Chapter 7}

\section{Muon decay and $\mathrm{W}$ mass}

Here we generalize our analysis from the previous chapter by considering the case of arbitrary mixings $e e_{m i x}, \mu \mu_{m i x}$ and $\tau \tau_{m i x}$. In this case non-SM box, vertex and selfenergy diagrams contributing to the muon decay (see Figs. 7.1- 7.4) may become important for the calculation of $M_{W}$. This is in contrast to the previous chapter where, as a result of the assumption $e e_{\text {mix }}=\mu \mu_{\text {mix }}=0$, only oblique corrections (corrections to the $\mathrm{W}$ propagator) had to be considered (see Sec. 6.5). Still, we assume in this chapter $e \mu_{m i x}=0$ and $\mu \tau_{m i x}=0$. The last parameter, $\mu \tau_{m i x}$ does not contribute to the muon decay. The neglect of $e \mu_{m i x}$ is supported by experiment (see Eq. 3.44) and the neglect of $\mu \tau_{m i x}$ is motivated by our intention to keep the discussion as straightforward as possible.

\subsection{Box diagrams}

The set of box diagrams contributing to the muon decay is depicted in Fig. 7.1.

As an example, we will calculate the diagrams of Fig. 7.1e. These two were chosen since, as we will show, they exhibit quadratic nondecoupling.

The amplitude for the diagram with the Higgs boson $H$ is given by (we sum over 


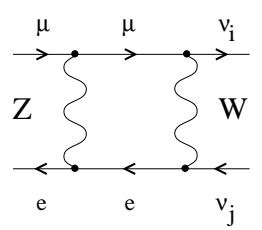

a)

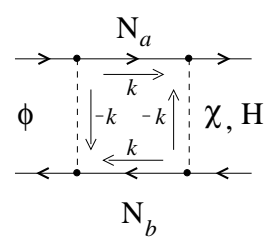

$e)$

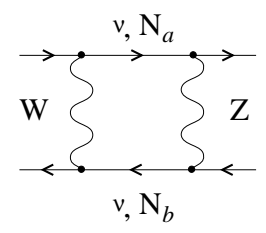

b)

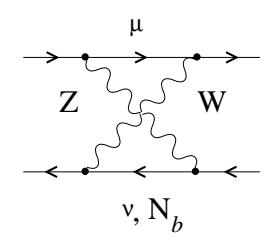

$f$ )

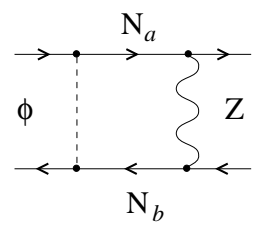

c)

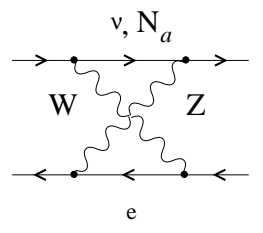

g)

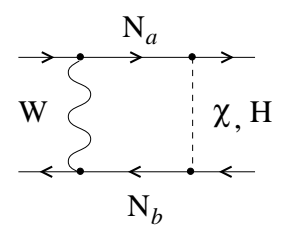

d)

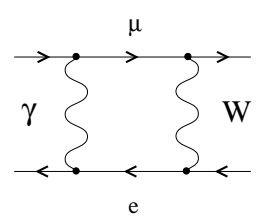

h)

Figure 7.1: Box diagrams for muon decay

NHL's $N_{a}, N_{b}$ with $M_{N_{a}}=M_{N_{b}}=M_{N}$ and we neglect external momenta in the internal propagators)

$$
\begin{aligned}
& \mathcal{M}_{\phi N H N}=\sum_{a, b} \int \frac{d k^{4}}{(2 \pi)^{4}} \overline{u_{\nu_{\mu}}} \frac{-i g_{2}}{2} \frac{M_{N}}{M_{W}}\left(K_{L}^{\dagger} K_{H}\right)_{i a} \frac{1+\gamma_{5}}{2} \frac{i}{\not k-M_{N}} \frac{i g_{2}}{2 \sqrt{2}} \frac{M_{N}}{M_{W}}\left(K_{H}^{\dagger}\right)_{a \mu} \\
& \times\left(1-\gamma_{5}\right) u_{\mu} \quad \overline{v_{e}} \frac{i g_{2}}{2 \sqrt{2}} \frac{M_{N}}{M_{W}}\left(K_{H}\right)_{e b}\left(1+\gamma_{5}\right) \frac{i}{\not k-M_{N}} \frac{-i g_{2}}{2} \frac{M_{N}}{M_{W}}\left(K_{H}^{\dagger} K_{L}\right)_{b j} \\
& \times \frac{1-\gamma_{5}}{2} v_{\nu_{e}} \frac{i}{k^{2}-M_{W}^{2}} \frac{i}{k^{2}-M_{H}^{2}} \\
& =\frac{g_{2}^{4}}{128} \frac{M_{N}^{4}}{M_{W}^{4}} \sum_{a, b}\left(K_{L}^{\dagger} K_{H}\right)_{i a}\left(K_{H}^{\dagger}\right)_{a \mu}\left(K_{H}\right)_{e b}\left(K_{H}^{\dagger} K_{L}\right)_{b j} \int \frac{d k^{4}}{(2 \pi)^{4}} \\
& \times \frac{\left[\overline{u_{\nu_{\mu}}}\left(1+\gamma_{5}\right)\left(\not k+M_{N}\right)\left(1-\gamma_{5}\right) u_{\mu}\right]\left[\overline{v_{e}}\left(1+\gamma_{5}\right)\left(\not k+M_{N}\right)\left(1-\gamma_{5}\right) v_{\nu_{e}}\right]}{\left(k^{2}-M_{N}^{2}\right)^{2}\left(k^{2}-M_{W}^{2}\right)\left(k^{2}-M_{H}^{2}\right)} \\
& =\frac{g_{2}^{4}}{32} \frac{M_{N}^{4}}{M_{W}^{4}} k_{m i x} \int \frac{d k^{4}}{(2 \pi)^{4}} \frac{\left[\overline{u_{\nu_{\mu}}}\left(1+\gamma_{5}\right) \not k u_{\mu}\right]\left[\overline{v_{e}}\left(1+\gamma_{5}\right) \not k v_{\nu_{e}}\right]}{\left(k^{2}-M_{N}^{2}\right)^{2}\left(k^{2}-M_{W}^{2}\right)\left(k^{2}-M_{H}^{2}\right)},
\end{aligned}
$$


where

$$
k_{m i x} \equiv\left(K_{L}^{\dagger} K_{H}\right)_{i a}\left(K_{H}^{\dagger}\right)_{a \mu}\left(K_{H}\right)_{e b}\left(K_{H}^{\dagger} K_{L}\right)_{b j}=\left(K_{L}^{\dagger}\right)_{i \mu}\left(K_{L}\right)_{e j} e e_{m i x} \mu \mu_{m i x}
$$

Using $\int k^{\epsilon} k^{\nu} \ldots=\frac{1}{4} g^{\epsilon \nu} \int k^{2} \ldots$ we get

$$
\begin{aligned}
\mathcal{M}_{\phi N H N} & =\frac{g_{2}^{4}}{32} \frac{M_{N}^{4}}{M_{W}^{4}} k_{m i x}\left[\overline{u_{\nu_{\mu}}}\left(1+\gamma_{5}\right) \gamma_{\epsilon} u_{\mu}\right]\left[\overline{v_{e}}\left(1+\gamma_{5}\right) \gamma_{\nu} v_{\nu_{e}}\right] \\
& \times \frac{1}{4} g^{\epsilon \nu} \frac{i}{(4 \pi)^{2}} \mathcal{I}_{2}\left(M_{H}\right)
\end{aligned}
$$

where $\mathcal{I}_{2}(m)$ is the integral

$$
\mathcal{I}_{2}(m)=\frac{(4 \pi)^{2}}{i} \int \frac{d^{4} k}{(2 \pi)^{4}} \frac{k^{2}}{\left(k^{2}-M_{N}^{2}\right)^{2}\left(k^{2}-M_{W}^{2}\right)\left(k^{2}-m^{2}\right)}
$$

In the next step we use the tree-level amplitude

$$
\mathcal{M}_{\text {tree }}=-\frac{i g_{2}^{2}}{8 M_{W}^{2}}\left[\overline{u_{\nu_{\mu}}}\left(1+\gamma_{5}\right) \gamma_{\alpha} u_{\mu}\right]\left[\overline{v_{e}}\left(1+\gamma_{5}\right) \gamma^{\alpha} v_{\nu_{e}}\right]\left(K_{L}^{\dagger}\right)_{i \mu}\left(K_{L}\right)_{e j}
$$

and $g_{2}=e / s_{W}$ to obtain

$$
\mathcal{M}_{\phi N H N}=-\frac{\alpha}{4 \pi} e e_{m i x} \mu \mu_{m i x} \frac{1}{16 s_{W}^{2}} \frac{M_{N}^{4}}{M_{W}^{2}} I_{2}\left(M_{H}\right) \mathcal{M}_{\text {tree }}
$$

The integral $\mathcal{I}_{2}\left(M_{H}\right)$ is calculated in Appendix $\mathrm{D}$. The result is

$$
\begin{aligned}
\mathcal{I}_{2}\left(M_{H}\right)= & \frac{1}{M_{H}^{2}-M_{W}^{2}}\left\{\frac{1}{1-\frac{M_{W}^{2}}{M_{N}^{2}}}+\frac{\frac{M_{W}^{4}}{M_{N}^{4}} \ln \frac{M_{W}^{2}}{M_{N}^{2}}}{\left(1-\frac{M_{W}^{2}}{M_{N}^{2}}\right)^{2}}-\frac{1}{1-\frac{M_{H}^{2}}{M_{N}^{2}}}\right. \\
& \left.-\frac{\frac{M_{H}^{4}}{M_{N}^{4}} \ln \frac{M_{H}^{2}}{M_{N}^{2}}}{\left(1-\frac{M_{H}^{2}}{M_{N}^{2}}\right)^{2}}\right\} .
\end{aligned}
$$

The amplitude $\mathcal{M}_{\phi N \chi N}$ is obtained from $\mathcal{M}_{\phi N H N}$ by replacing $M_{H}$ with $M_{Z}$. 
The total contribution of the box diagrams (Figs. $7.1 \mathrm{a}-\mathrm{g}$ ) is

$$
\begin{aligned}
\mathcal{M}_{\text {box }} & =\mathcal{M}_{Z e W \mu}+\mathcal{M}_{W \nu Z \nu}+\mathcal{M}_{W \nu Z N}+\mathcal{M}_{W N Z \nu}+\mathcal{M}_{W N Z N} \\
& +\mathcal{M}_{\phi N Z N}+\mathcal{M}_{W N H N}+\mathcal{M}_{W N \chi N}+\mathcal{M}_{\phi N H N}+\mathcal{M}_{\phi N \chi N} \\
& +\mathcal{M}_{Z \nu W \mu}+\mathcal{M}_{Z N W \mu}+\mathcal{M}_{W e Z \nu}+\mathcal{M}_{W e Z N} \\
& =\mathcal{M}_{\text {tree }} \frac{\alpha}{4 \pi}\left\{\frac { - 1 } { 4 s _ { W } ^ { 2 } c _ { W } ^ { 2 } } M _ { W } ^ { 2 } \left[4\left(-\frac{1}{2}+s_{W}^{2}\right)^{2} \mathcal{I}_{0}+\mathcal{I}_{0}\left(1-\mu \mu_{\text {mix }}\right)\left(1-e e_{m i x}\right)\right.\right. \\
& +\mathcal{I}_{1}\left(M_{Z}\right)\left(1-\mu \mu_{m i x}\right) e e_{\text {mix }}+\mathcal{I}_{1}\left(M_{Z}\right) \mu \mu_{m i x}\left(1-e e_{m i x}\right)+\mathcal{I}_{2}\left(M_{Z}\right) e e_{m i x} \\
& \left.\times \mu \mu_{m i x}\right]+\frac{1}{4 s_{W}^{2}} M_{N}^{4}\left[\frac{1}{c_{W}^{2}} \mathcal{I}_{3}\left(M_{Z}\right)+\mathcal{I}_{3}\left(M_{H}\right)+\mathcal{I}_{3}\left(M_{Z}\right)-\frac{1}{4 M_{W}^{2}} \mathcal{I}_{2}\left(M_{H}\right)\right. \\
& \left.-\frac{1}{4 M_{W}^{2}} \mathcal{I}_{2}\left(M_{Z}\right)\right] e e_{m i x} \mu \mu_{m i x}+\frac{2\left(-\frac{1}{2}+s_{W}^{2}\right)}{s_{W}^{2} c_{W}^{2}} M_{W}^{2}\left[\mathcal{I}_{0}\left(1-e e_{m i x}\right)\right. \\
& \left.\left.+\mathcal{I}_{1}\left(M_{Z}\right) e e_{m i x}+\mathcal{I}_{0}\left(1-\mu \mu_{m i x}\right)+\mathcal{I}_{1}\left(M_{Z}\right) \mu \mu_{m i x}\right]\right\}
\end{aligned}
$$

where the integrals $\mathcal{I}_{0}, \mathcal{I}_{1}(m), \mathcal{I}_{3}(m)$ are

$$
\begin{aligned}
\mathcal{I}_{0} & =\frac{(4 \pi)^{2}}{i} \int \frac{d^{4} k}{(2 \pi)^{4}} \frac{1}{k^{2}\left(k^{2}-M_{W}^{2}\right)\left(k^{2}-M_{Z}^{2}\right)}=\frac{1}{M_{Z}^{2}-M_{W}^{2}} \ln \frac{M_{W}^{2}}{M_{Z}^{2}} \\
\mathcal{I}_{1}(m) & =\frac{(4 \pi)^{2}}{i} \int \frac{d^{4} k}{(2 \pi)^{4}} \frac{1}{\left(k^{2}-M_{N}^{2}\right)\left(k^{2}-M_{W}^{2}\right)\left(k^{2}-m^{2}\right)} \\
& =\frac{1}{m^{2}-M_{W}^{2}}\left\{\ln \frac{M_{W}^{2}}{m^{2}}+\frac{M_{N}^{2}}{M_{W}^{2}-M_{N}^{2}} \ln \frac{M_{W}^{2}}{M_{N}^{2}}-\frac{M_{N}^{2}}{m^{2}-M_{N}^{2}} \ln \frac{m^{2}}{M_{N}^{2}}\right\} \\
\mathcal{I}_{3}(m) & =\frac{(4 \pi)^{2}}{i} \int \frac{d^{4} k}{(2 \pi)^{4}} \frac{1}{\left(k^{2}-M_{N}^{2}\right)^{2}\left(k^{2}-M_{W}^{2}\right)\left(k^{2}-m^{2}\right)} \\
& =\frac{1}{m^{2}-M_{W}^{2}}\left\{\frac{1}{M_{N}^{2}-M_{W}^{2}}+\frac{M_{W}^{2} \ln \frac{M_{W}^{2}}{M_{N}^{2}}}{\left(M_{N}^{2}-M_{W}^{2}\right)^{2}}-\frac{1}{M_{N}^{2}-m^{2}}\right.
\end{aligned}
$$




$$
\left.-\frac{m^{2} \ln \frac{m^{2}}{M_{N}^{2}}}{\left(M_{N}^{2}-m^{2}\right)^{2}}\right\}
$$

For the calculation of these integrals see Appendix D. In the limit of $M_{N} \gg M_{W}$, the leading contribution to $\mathcal{I}_{2}(m)$ is very simple,

$$
\mathcal{I}_{2}^{a p p x}(m)=-\frac{1}{M_{N}^{2}}
$$

implying the $M_{N}^{2}$ dependence of the $\mathcal{M}_{\phi N H N}$ and $\mathcal{M}_{\phi N \chi N}$,

$$
\mathcal{M}_{\phi N H N}^{a p p x}=\mathcal{M}_{t r e e} \frac{\alpha}{4 \pi} \frac{1}{16 s_{W}^{2}} \frac{M_{N}^{2}}{M_{W}^{2}} e e_{m i x} \mu \mu_{m i x}
$$

The leading contribution to $\mathcal{I}_{3}(m)$ goes as $\sim 1 / M_{N}^{4}$ and to $\mathcal{I}_{1}(m)$ as $\sim 1 / M_{N}^{2}$, therefore no other box depends quadratically on the NHL mass.

The last box diagram, Fig. 7.1 h can be written as [34]:

$$
\mathcal{M}_{\gamma e W \mu}=\mathcal{M}_{\text {tree }} \frac{\alpha}{4 \pi}\left(\ln \frac{M_{W}}{m_{e}}+\ln \frac{M_{W}}{m_{\mu}}-2 \ln \frac{m_{e}}{\lambda}-2 \ln \frac{m_{\mu}}{\lambda}+\frac{9}{2}\right)+\ldots
$$

The ellipses denote additional terms discussed in Ref. [34].

\subsection{Neutrino self-energy and its renormalization}

One half of the neutrino self-energy diagrams contributing to muon decay is shown in Fig. 7.2. The corresponding self-energy is denoted as $\Sigma^{\nu_{\mu}}$. The other half consists of the same loops sitting on the bottom neutrino leg with the corresponding self-energy $\Sigma^{\nu_{e}}$. In all these diagrams, we sum over the internal massless neutrinos $\nu_{k}, k=1,2,3$. In principle, the graphs with $\nu_{k}$ replaced by $N_{a}$ are also present, however, they are suppressed by the large mass $M_{N}$.

Without derivation, we present results for the unrenormalized neutrino self- 


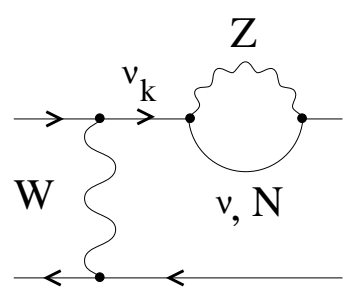

a)

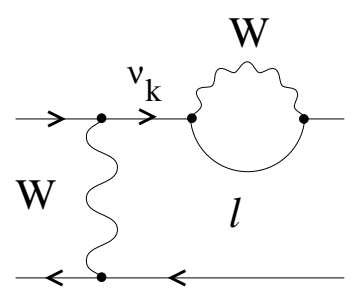

b)

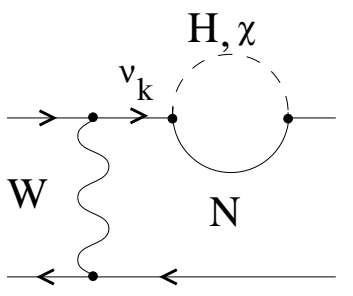

c)

Figure 7.2: Neutrino self-energy diagrams for muon decay

energy $\Sigma^{\nu_{l}}(l=e, \mu)$. It has the form

$$
\Sigma^{\nu_{l}}=\frac{1}{2} \Sigma_{L}^{\nu_{l}} \not p\left(1-\gamma_{5}\right),
$$

where $\Sigma_{L}^{\nu_{l}}$ receives the following contributions from the diagrams of Fig. 7.2:

$$
\begin{aligned}
\Sigma_{L}^{\nu_{l}} & =\Sigma_{L}^{H}(p)+\Sigma_{L}^{\chi}(p)+\Sigma_{L}^{Z, N}(p)+\Sigma_{L}^{Z, \nu}(p)+\Sigma_{L}^{W}(p) \\
& =\frac{\alpha}{2 \pi}\left(1-l l_{\text {mix }}\right)\left\{\frac{1}{8 s_{W}^{2}} l l_{\text {mix }} \frac{M_{N}^{2}}{M_{W}^{2}}\left[\frac{1}{2} \Delta_{\mu}+B_{0}^{f i n}\left(p ; M_{H}, M_{N}\right)+B_{1}^{f i n}\left(p ; M_{H}, M_{N}\right)\right]\right. \\
& +\frac{1}{8 s_{W}^{2}} l l_{m i x} \frac{M_{N}^{2}}{M_{W}^{2}}\left[\frac{1}{2} \Delta_{\mu}+B_{0}^{f i n}\left(p ; M_{Z}, M_{N}\right)+B_{1}^{f i n}\left(p ; M_{Z}, M_{N}\right)\right] \\
& +\frac{1}{4 s_{W}^{2} c_{W}^{2}} l l_{m i x}\left[\frac{1}{2} \Delta_{\mu}-\frac{1}{2}+B_{0}^{f i n}\left(p ; M_{Z}, M_{N}\right)+B_{1}^{f i n}\left(p ; M_{Z}, M_{N}\right)\right] \\
& +\frac{1}{4 s_{W}^{2} c_{W}^{2}}\left(1-l l_{m i x}\right)\left[\frac{1}{2} \Delta_{\mu}-\frac{1}{2}+B_{0}^{f i n}\left(p ; M_{Z}, 0\right)+B_{1}^{f i n}\left(p ; M_{Z}, 0\right)\right] \\
& \left.+\frac{1}{2 s_{W}^{2}}\left[\frac{1}{2} \Delta_{\mu}-\frac{1}{2}+B_{0}^{f i n}\left(p ; M_{W}, m_{l} \rightarrow 0\right)+B_{1}^{f i n}\left(p ; M_{W}, m_{l} \rightarrow 0\right)\right]\right\} .
\end{aligned}
$$

Here $s=p^{2}=0 \ll M_{H}^{2}, M_{Z}^{2}, M_{W}^{2}, M_{N}^{2}$, therefore Eqs. E.18 apply for $B_{0}^{\text {fin }}, B_{1}^{f i n}$. The amplitude for the diagrams of Fig. 7.2 in terms of $\Sigma_{L}^{\nu_{l}}$ can be shown to be 
equal to

$$
\mathcal{M}_{\text {self }}=-\mathcal{M}_{\text {tree }} \frac{\Sigma_{L}^{\nu_{l}}}{2}
$$

where the one half comes from our dealing with the external wave function rather than the neutrino propagator.

\subsubsection{Renormalization of the neutrino self-energies}

Let us now investigate the question of the renormalization of the diagrams of Fig. 7.2 . It is the only case in this work when the counterterms are modified from their SM form . The problem is how to renormalize a part of a theory where interaction eigenstates are different from mass eigenstates. Curiously, this also happens in the SM quark sector. The difference is that in the SM the problem is circumvented by arguing the off-diagonal quark mixings are too small (with the required accuracy in mind) to have any effect in the loops and the renormalization procedure is effectively simplified to that of mass eigenstates being also the flavour eigenstates. In our model, we cannot neglect the off-diagonal mixings $\left(l l_{m i x}\right)$, since they (in combination with TeV NHL masses) lead to the dominant terms in the predicted signals 2 .

To derive the required counterterm, we start with the counterterm Lagrangian, which has the same form in both SM and our model:

$$
i \delta Z_{L}^{e} \overline{\nu_{e}} \not \partial \nu_{e}+i \delta Z_{L}^{\mu} \overline{\nu_{\mu}} \not \partial \nu_{\mu}+i \delta Z_{L}^{\tau} \overline{\nu_{\tau}} \not \partial \nu_{\tau}
$$

where $\delta Z_{L}^{l}$ is the sum of $\delta Z_{V}^{l}$ and $\delta Z_{A}^{l}$ renormalization constants which are given

\footnotetext{
${ }^{1}$ So far we have used the SM form of the counterterms, see Appendix E.1. The actual value of the counterterms was, of course, different from SM.

${ }^{2}$ Just a few days before the submission of this thesis an interesting paper appeared on the preprint bulletin board [64], where the arguments of this paragraph are also made. The authors then continue to develop the first formal framework ever for the renormalization of theories with nonnegligible mixings between mass and interaction eigenstates. Their treatment is more general than ours below.
} 
in Eq. E.4. Weak eigenstates $\nu_{l}$ can be expressed in our model in terms of mass eigenstates $\nu_{i}, N_{a}$ (see Eq. 3.23)

$$
\begin{aligned}
\nu_{l} & =\sum_{i}\left(K_{L}\right)_{l i} \nu_{i}+\sum_{a}\left(K_{H}\right)_{l a} N_{a}=\sum_{n_{\alpha}=\nu_{1}, \ldots, N_{6}} K_{l n_{\alpha}} n_{\alpha}, \\
\overline{\nu_{l}} & =\overline{n_{\alpha}} K_{n_{\alpha} l}^{\dagger} .
\end{aligned}
$$

This gives us for the product $\overline{\nu_{l}} \nu_{l}$

$$
\overline{\nu_{l}} \nu_{l}=\bar{n} K^{\dagger} K n=\sum_{k, i=1,2,3} \overline{\nu_{i}}\left(K_{L}^{\dagger}\right)_{i l}\left(K_{L}\right)_{l k} \nu_{k}+\ldots\left(\overline{\nu_{i}} N, \bar{N} \nu_{k}, \bar{N} N\right)
$$

and Eq. 7.18 thus contributes the following terms as the massless neutrino counterterm Lagrangians:

$$
\sum_{k, i=1,2,3}\left\{\delta Z_{L}^{e}\left(K_{L}^{\dagger}\right)_{i e}\left(K_{L}\right)_{e k}+\delta Z_{L}^{\mu}\left(K_{L}^{\dagger}\right)_{i \mu}\left(K_{L}\right)_{\mu k}+\delta Z_{L}^{\tau}\left(K_{L}^{\dagger}\right)_{i \tau}\left(K_{L}\right)_{\tau k}\right\} \overline{\nu_{i}} \not \partial \nu_{k}
$$

In our case, however, we sum over internal $\nu_{k}$ but not over external $\nu_{i}$. The graphic representation of the relevant counterterm (embedded in muon decay) is in Fig. 7.3 . The amplitude for this diagram is

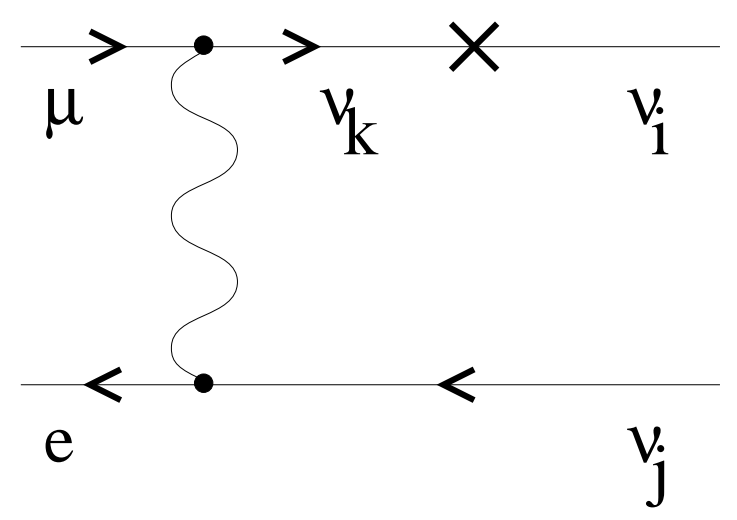

Figure 7.3: Counterterm diagram for neutrino self-energy in muon decay 


$$
\mathcal{M}_{C}=-\frac{1}{2} \mathcal{M}_{\text {tree }}^{S M} \sum_{l=e, \mu, \tau} \sum_{k=1,2,3} \delta Z_{L}^{l}\left(K_{L}^{\dagger}\right)_{i l}\left(K_{L}\right)_{l k}\left(K_{L}^{\dagger}\right)_{k \mu}
$$

Again, the factor $\frac{1}{2}$ comes from our dealing with the external wave function rather than the internal propagator and the mixing factor $\left(K_{L}^{\dagger}\right)_{k \mu}$ originates at the $\mu W \nu_{k}$ vertex. The amplitude $\mathcal{M}_{C}$ can be further simplified,

$$
\begin{aligned}
\mathcal{M}_{C} & =-\frac{1}{2} \mathcal{M}_{\text {tree }}^{S M} \sum_{l=e, \mu, \tau} \delta Z_{L}^{l}\left(K_{L}^{\dagger}\right)_{i l} \sum_{k=1,2,3}\left(K_{L}\right)_{l k}\left(K_{L}^{\dagger}\right)_{k \mu} \\
& =-\frac{1}{2} M_{\text {tree }}^{S M} \sum_{l=e, \mu, \tau} \delta Z_{L}^{l}\left(K_{L}^{\dagger}\right)_{i l}\left(\delta_{l \mu}-l \mu_{\text {mix }}\right)=-\frac{1}{2} M_{\text {tree }}^{S M} \delta Z_{L}^{\mu}\left(1-\mu \mu_{\text {mix }}\right)\left(K_{L}^{\dagger}\right)_{i \mu} \\
& =-\frac{1}{2} \delta Z_{L}^{\mu}\left(1-\mu \mu_{\text {mix }}\right) \mathcal{M}_{\text {tree }} .
\end{aligned}
$$

The factor $\left(K_{L}^{\dagger}\right)_{i \mu}$ was absorbed by $M_{\text {tree }}=M_{\text {tree }}^{S M}\left(K_{L}^{\dagger}\right)_{i \mu}$.

Now we can write down the final expressions for the renormalized amplitude $\hat{\mathcal{M}}_{\text {self }}$ and the renormalized neutrino self-energy:

$$
\begin{aligned}
\hat{\mathcal{M}}_{\text {self }} & =\mathcal{M}_{\text {self }}+\mathcal{M}_{C}=-\frac{\Sigma_{L}^{\nu_{l}}}{2} \mathcal{M}_{\text {tree }}-\frac{\delta Z_{L}^{l}}{2}\left(1-l l_{\text {mix }}\right) \mathcal{M}_{\text {tree }} \\
\hat{\Sigma}_{L}^{\nu_{l}} & =\Sigma_{L}^{\nu_{l}}+\delta Z_{L}^{l}\left(1-l l_{\text {mix }}\right) .
\end{aligned}
$$

The constant $\delta Z_{L}^{l}$ is found from

$$
\begin{aligned}
\delta Z_{L}^{l} & =-\Sigma_{L}^{l}\left(m_{l}^{2}\right)-m_{l}^{2}\left[\Sigma_{L}^{l^{\prime}}\left(m_{l}^{2}\right)+\Sigma_{R}^{l^{\prime}}\left(m_{l}^{2}\right)+2 \Sigma_{S}^{l^{\prime}}\left(m_{l}^{2}\right)\right] \\
\Sigma_{L, R, S}^{l^{\prime}}\left(m_{l}^{2}\right) & =\frac{\partial \Sigma_{L, R, S}^{l}}{\partial p^{2}}\left(m_{l}^{2}\right)
\end{aligned}
$$

where $\Sigma_{L}, \Sigma_{R}$ and $\Sigma_{S}$ are respectively the left-handed, right-handed and the scalar part of the lepton self-energy given in Eqs. 6.41 - 6.44 9. The only graph with significant contribution to the term with derivatives, is the photonic loop (Eq. 6.44).

\footnotetext{
${ }^{3}$ Now we include the photonic loop $\Sigma_{\gamma l}^{l}$ in the total lepton self-energy, see Eq. 6.44 and the following comments.
} 
The other diagrams lead to derivatives of $B_{0}^{\text {fin }}, B_{1}^{\text {fin }}$ functions which, when multiplied by $m_{l}^{2}$, are of the order $m_{l}^{2} / M_{W}^{2}$ and therefore negligible. This can be easily seen from Eqs. E.18, E.19, wherein also derivatives of the $B_{0}^{\text {fin }}, B_{1}^{\text {fin }}$ functions corresponding to the photonic loop are given. The final answer is

$$
\delta Z_{L}^{l}=-\Sigma_{L}^{l}\left(m_{l}^{2}\right)+\frac{\alpha}{2 \pi}\left(2 \ln \frac{m_{l}}{\lambda}-1\right)
$$

To prove the cancellation of the infinities, we note that the infinite part of $\delta Z_{L}^{l}$ is given by

$$
\delta Z_{L}^{l, \infty}=-\frac{\alpha}{4 \pi} \frac{1}{s_{W}^{2}}\left\{\frac{1}{2}+\frac{1}{4 c_{W}^{2}}+\frac{\mathcal{X}}{4} l l_{m i x}\right\} \Delta_{\mu}
$$

and the infinite part of the neutrino self-energy by

$$
\begin{aligned}
\Sigma_{L}^{\nu_{l}, \infty} & =\frac{\alpha}{4 \pi} \frac{1}{s_{W}^{2}}\left\{\frac{\mathcal{X}}{4} l l_{m i x}\left(1-l l_{\text {mix }}\right)+\frac{1}{2}\left(1-l l_{\text {mix }}\right)+\frac{1}{4 c_{W}^{2}} l l_{m i x}\left(1-l l_{\text {mix }}\right)\right. \\
& \left.+\frac{1}{4 c_{W}^{2}}\left(1-l l_{\text {mix }}\right)^{2}\right\} \Delta_{\mu} .
\end{aligned}
$$

From the formulae above it can be easily seen that infinities cancel out in Eq. 7.25.

\subsubsection{Limit $M_{N} \gg M_{W}, M_{Z}, M_{H}$}

We will investigate the large $M_{N}$ behaviour of the renormalized neutrino self-energy $\hat{\Sigma}_{L}^{\nu_{l}}$. For large $M_{N}$ we get from Eq. E.18

$$
\begin{aligned}
& B_{0}\left(p ; M_{H, Z, W}, M_{N}\right)=1-2 \ln M_{N} \\
& B_{1}\left(p ; M_{H, Z, W}, M_{N}\right)=-0.25+\ln M_{N} .
\end{aligned}
$$


This implies the quadratic nondecoupling for $\Sigma_{L}^{H}(p)$ and $\Sigma_{L}^{\chi}(p)$, see Eq. 7.16:

$$
\Sigma_{L}^{H}(p)+\Sigma_{L}^{\chi}(p)=\frac{\alpha}{2 \pi} \frac{1}{4 s_{W}^{2}} l l_{m i x}\left(1-l l_{m i x}\right) \frac{M_{N}^{2}}{M_{W}^{2}}\left[\frac{1}{2} \Delta_{\mu}+0.75-\ln M_{N}\right]
$$

and for the $\Sigma_{L}^{\phi N}$, a left-handed part of $\Sigma_{\phi N}^{l}$ (see Eq. 6.43), which contributes to the $\hat{\Sigma}_{L}^{\nu_{l}}$ via the counterterm $\delta Z_{L}^{l}$, see Eq. 7.27:

$$
\Sigma_{L}^{\phi N}=+\frac{\alpha}{16 \pi s_{W}^{2}} l l_{m i x} \frac{M_{N}^{2}}{M_{W}^{2}}\left[\Delta_{\mu}+\frac{3}{2}-2 \ln M_{N}\right]
$$

From here we can see the $\Sigma_{L}^{\phi N}$ not only cancels out infinities in the $\Sigma_{L}^{H}(p)$ and $\Sigma_{L}^{\chi}(p)$, but, in the limit investigated, it also cancels out the finite parts. As a result, there is no quadratic nondecoupling in the renormalized neutrino self-energy.

\subsection{Vertex diagrams}

Diagrams modifying the $W \mu \nu_{i}$ vertex are depicted in Fig. 7.4. Another set, one that modifies $W e \nu_{j}$ vertex, is not shown.

The sum over the depicted set of diagrams gives the muon vertex amplitude $\mathcal{M}_{\text {vertex }}^{\mu}$

$$
\begin{aligned}
\mathcal{M}_{\text {vertex }}^{\mu} & =\mathcal{M}_{\mu \nu Z}+\mathcal{M}_{\mu N Z}+\mathcal{M}_{Z W \mu}+\mathcal{M}_{\gamma W \mu}+\mathcal{M}_{W Z \nu} \\
& +\mathcal{M}_{W Z N}+\mathcal{M}_{\phi Z N}+\mathcal{M}_{W H N}+\mathcal{M}_{\phi H N}+\mathcal{M}_{\phi \chi N}
\end{aligned}
$$

The computation of the diagrams yields

$$
\begin{aligned}
\mathcal{M}_{\text {vertex }}^{\mu} & =\mathcal{M}_{\text {tree }} \frac{\alpha}{4 \pi}\left\{\frac{2 s_{W}^{2}-1}{4 s_{W}^{2} c_{W}^{2}}\left(\Delta_{M_{Z}}-\frac{1}{2}\right)\left(1-\mu \mu_{\text {mix }}\right)\right. \\
& +\frac{2 s_{W}^{2}-1}{4 s_{W}^{2} c_{W}^{2}}\left(\Delta_{M_{Z}}-\frac{1}{2}-\frac{M_{N}^{2}}{M_{Z}^{2}-M_{N}^{2}} \ln \frac{M_{Z}^{2}}{M_{N}^{2}}\right) \mu \mu_{\text {mix }}
\end{aligned}
$$




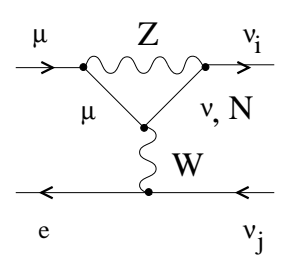

a)

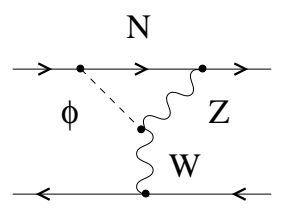

d)

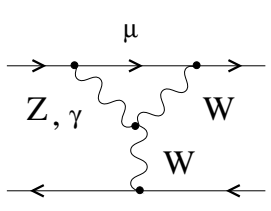

b)

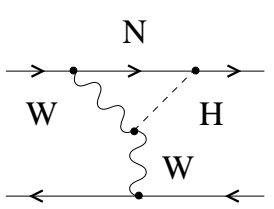

e)

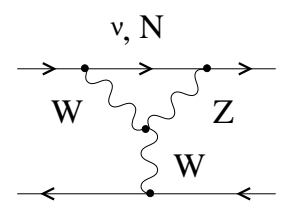

c)

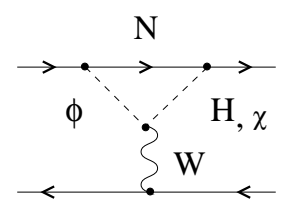

f)

Figure 7.4: Vertex diagrams for muon decay

$$
\begin{aligned}
& +\frac{\frac{1}{2}-s_{W}^{2}}{s_{W}^{2}}\left(3 \Delta_{M_{W}}+\frac{5}{2}+\frac{3}{s_{W}^{2}} \ln c_{W}^{2}\right)+3\left(\Delta_{M_{W}}+\frac{5}{6}\right) \\
& +\frac{3}{2 s_{W}^{2}}\left(\Delta_{M_{W}}+\frac{5}{6}+\frac{1}{s_{W}^{2}} \ln c_{W}^{2}\right)\left(1-\mu \mu_{m i x}\right) \\
& +\frac{3}{2 s_{W}^{2}}\left[\Delta_{M_{W}}+\frac{5}{6}+\frac{1}{s_{W}^{2}} \ln c_{W}^{2}+\frac{M_{N}^{2}}{M_{Z}^{2}-M_{W}^{2}} v\left(M_{Z}\right)\right] \mu \mu_{m i x} \\
& +\frac{1}{2 c_{W}^{2}} \frac{-M_{N}^{2}}{M_{Z}^{2}-M_{W}^{2}} v\left(M_{Z}\right) \mu \mu_{m i x} \\
& +\frac{1}{2 s_{W}^{2}} \frac{-M_{N}^{2}}{M_{H}^{2}-M_{W}^{2}} v\left(M_{H}\right) \mu \mu_{m i x} \\
& +\frac{1}{8 s_{W}^{2}} \frac{M_{N}^{2}}{M_{W}^{2}}\left[\Delta_{M_{W}}+\frac{3}{2}-\frac{M_{H}^{2}}{M_{W}^{2}-M_{H}^{2}} \ln \frac{M_{W}^{2}}{M_{H}^{2}}+\frac{M_{N}^{2}}{M_{H}^{2}-M_{W}^{2}} v\left(M_{H}\right)\right] \mu \mu_{m i x} \\
& +\frac{1}{8 s_{W}^{2}} \frac{M_{N}^{2}}{M_{W}^{2}}\left[\Delta_{M_{W}}+\frac{3}{2}-\frac{M_{Z}^{2}}{M_{W}^{2}-M_{Z}^{2}} \ln \frac{M_{W}^{2}}{M_{Z}^{2}}+\frac{M_{N}^{2}}{M_{Z}^{2}-M_{W}^{2}} v\left(M_{Z}\right)\right] \\
& \left.\times \mu \mu_{m i x}\right\},
\end{aligned}
$$


where

$$
v(m)=\ln \frac{M_{W}^{2}}{m^{2}}+\frac{M_{N}^{2}}{M_{W}^{2}-M_{N}^{2}} \ln \frac{M_{W}^{2}}{M_{N}^{2}}-\frac{M_{N}^{2}}{m^{2}-M_{N}^{2}} \ln \frac{m^{2}}{M_{N}^{2}} .
$$

The vertices are renormalized as (see Eq. E.3)

$$
\hat{\Lambda}^{\mu}=\Lambda^{\mu}+\delta Z_{1}^{W}-\delta Z_{2}^{W}+\delta Z_{L}^{\mu}
$$

where

$$
\begin{aligned}
\Lambda^{\mu} & =\mathcal{M}_{\text {vertex }}^{\mu} / \mathcal{M}_{\text {tree }} \\
\delta Z_{1}^{W}-\delta Z_{2}^{W} & =-\frac{\alpha}{2 \pi s_{W}^{2}} \Delta_{M_{W}}
\end{aligned}
$$

Looking for the dominant graphs in the limit $M_{N} \gg M_{W}, M_{Z}, M_{H}$, we note the leading contribution to the function $v(m)$ is

$$
v(m)^{a p p x}=\frac{1}{M_{N}^{2}}\left(m^{2} \ln \frac{m^{2}}{M_{N}^{2}}-M_{W}^{2} \ln \frac{M_{W}^{2}}{M_{N}^{2}}\right),
$$

which implies the graphs of Fig. 7.4f have quadratic nondecoupling f. However, as in the case of the neutrino self-energy, both infinite and finite terms of these graphs are cancelled by the $\Sigma_{L}^{\phi N}$ term in the counterterm $\delta Z_{L}^{l}$. Therefore there are no $M_{N}^{2}$ dependent terms in the renormalized vertex diagrams either.

\footnotetext{
${ }^{4}$ Another interesting point the authors of Ref. [64 make (see also footnote 2 on page 122) is that the diagrams for the muon decay ( $\pi$ decay in Ref. 64), Figs. 7.1e, 7.2 c, 7.4f can be singled out as dominant in an elegant way by using the Goldstone boson equivalence theorem.
} 


\subsection{Results}

The muon decay loops modify the $\Delta r$ quantity in the implicit relation between $M_{W}$ and $G_{\mu}$, see Eq. 6.61:

$$
M_{W}^{2} s_{W}^{2}=\frac{\pi \alpha}{\sqrt{2} G_{\mu}(1-\Delta r)}\left(1-\frac{1}{2} e e_{m i x}-\frac{1}{2} \mu \mu_{m i x}\right)
$$

and $\Delta r$ can be written as (see Eq. 6.62)

$$
\Delta r=\frac{\mathcal{R} e \hat{\Sigma}_{W}(0)}{M_{W}^{2}}+\delta_{V}
$$

Here the parameter $\delta_{V}$ is the sum of the loop diagrams calculated in this section:

$$
\delta_{V}=\frac{\mathcal{M}_{\gamma e W \mu}}{\mathcal{M}_{\text {tree }}}+\hat{\Lambda}^{\mu}+\hat{\Lambda}^{e}-\frac{1}{2} \hat{\Sigma}^{\nu_{e}}-\frac{1}{2} \hat{\Sigma}^{\nu_{\mu}}+\frac{\mathcal{M}_{\text {box }}}{\mathcal{M}_{\text {tree }}}
$$

Numerical results are shown in Table 7.1. As input data we used masses from the standard set, mixings $e e_{m i x}=0.0071, \mu \mu_{m i x}=0.0014$ and $\tau \tau_{m i x}=0$. We suppressed the $\tau \tau_{m i x}$, which at its maximal value currently allowed (0.033) would make corrections to $\hat{\Sigma}_{W}(0) / M_{W}^{2}$ much larger than the corrections to $\delta_{V}$ (these only depend on $\left.e e_{m i x}, \mu \mu_{m i x}\right)$ and we would like to show the case when also the latter are important. In the first three lines of the table we show how much the self-energy, vertex and box diagrams contribute to $\delta_{V}$ (line 4) for NHL masses $M_{N}$ of up to 30

TeV. Also shown (lines 5,6) are $\hat{\Sigma}_{W}(0) / M_{W}^{2}$ and $\Delta r$. Ultimately we are interested in NHL effects in the observable $M_{W}$ (line 7).

The results confirm expectations from the previous sections. There is no nondecoupling for self-energies and vertices and there is a quadratic dependence on $M_{N}$ for the boxes. The boxes are becoming important at very high masses. Still, they are small compared to the change in $\hat{\Sigma}_{W}(0) / M_{W}^{2}$. This is due to the fact the dominant boxes depend on the product $e e_{\text {mix }} \mu \mu_{\text {mix }}$ (see Eq. 7.13), while the correction 


\begin{tabular}{|l|r|r|r|r|r|r|}
\hline & $\mathrm{SM}$ & $0.5 \mathrm{TeV}$ & $5 \mathrm{TeV}$ & $15 \mathrm{TeV}$ & $30 \mathrm{TeV}$ & \\
\hline$\hat{\Sigma}^{\nu_{e}}+\hat{\Sigma}^{\nu_{\mu}}$ & -4.995 & -4.972 & -4.982 & -4.988 & -4.992 & $\times 10^{-2}$ \\
$\hat{\Lambda}^{\mu}$ & -1.441 & -1.442 & -1.444 & -1.444 & -1.445 & $\times 10^{-2}$ \\
$\mathcal{M}_{\text {box }} / \mathcal{M}_{\text {tree }}$ & 4.273 & 4.300 & 4.315 & 4.457 & 4.950 & $\times 10^{-3}$ \\
$\delta_{V}$ & 6.670 & 6.539 & 6.525 & 6.652 & 7.133 & $\times 10^{-3}$ \\
$\hat{\Sigma}_{W}(0) / M_{W}^{2}$ & 2.396 & 2.346 & 2.301 & 1.872 & 0.329 & $\times 10^{-2}$ \\
$\Delta r$ & 3.063 & 3.000 & 2.954 & 2.537 & 1.043 & $\times 10^{-2}$ \\
$M_{W}$ & 80.459 & 80.537 & 80.545 & 80.612 & 80.846 & $\times 1$ \\
\hline
\end{tabular}

Table 7.1: Contribution of the muon decay loops to $\delta_{V}$ and $\Delta_{r}$

to the $W$ propagator is proportional to $k_{H H}=e e_{m i x}^{2}+\mu \mu_{m i x}^{2}$ (see Eq. 6.25), which is allowed to be larger given the current bounds on the mixings.

The $W$ mass jumps from $M_{W}^{S M}=80.459 \mathrm{GeV}$ to $M_{W}=80.537 \mathrm{GeV}$ at $M_{N}=0.5$ $\mathrm{TeV}$ mainly as a result of the tree-level correction $\left(1-\frac{1}{2} e e_{m i x}-\frac{1}{2} \mu \mu_{m i x}\right)$, see Eq. 7.40. After that it rises very slowly until the $M_{N}$ dependent amplitudes become dominant above $5 \mathrm{TeV}$.

In conclusion, the numerical analysis of Chapter 6 turns out to be basically valid even after the restriction $e e_{\text {mix }}=\mu \mu_{\text {mix }}=0$ is relaxed. It can be improved by the inclusion of the tree-level correction $\left(1-\frac{1}{2} e e_{m i x}-\frac{1}{2} \mu \mu_{m i x}\right)$, while the largest loop corrections, the box diagrams of Fig. 7.1e, are only marginally important. 


\section{Chapter 8}

\section{Conclusions}

Two theoretical schemes were discussed here as possible solutions of the problem of the small neutrino masses: the see-saw mechanism of Yanagida and Gell-Mann, Ramond and Slansky [8, 9] (Sec. 2.3.2); and the superstring-inspired low-energy model of neutrino masses suggested in Refs. [17, 27] (Chapter 3). Both these solutions introduce NHL's into the theory as a necessary ingredient. Our main consideration has been the phenomenology of NHL's in the superstring-inspired lowenergy model. The qualitative features of our analysis are applicable also in the context of see-saw models with enhanced mixings.

The superstring-inspired low-energy model (Chapter 3) is a simple extension of the SM. It enriches only the neutral fermion spectrum of the SM, leaving the gauge and Higgs sectors intact. We found that among the new parameters of the model, seven are especially important: 'flavour-conserving' mixing parameters $e e_{m i x}, \mu \mu_{m i x}, \tau \tau_{m i x}$; 'flavour-violating' mixing parameters $e \mu_{m i x}, e \tau_{m i x}, \mu \tau_{m i x}$ and the mass scale $M_{N}$ of NHL's (we assumed all three NHL's have mass $M_{N}$ ). The bounds on the six mixing parameters (Sec. 3.4) are largely independent of the mass $M_{N}$. On the other hand bounds on $M_{N}$ always depend on the mixings.

The mass $M_{N}$, if larger than $M_{Z}$, can presently only be probed in radiative 
corrections (loops). A traditional approach was mostly limited to hypothetical lepton flavour-violating processes such as $\mu \rightarrow e \gamma ; \mu, \tau \rightarrow e e^{+} e^{-} ; Z \rightarrow e^{ \pm} \mu^{\mp}$ etc [20, 42, 26, 43, 44, 45, 46, 47, 48. We reviewed constraints from these processes in Chapter 5.

Besides flavour-violating processes NHL's could also induce (via radiative corrections) deviations from the SM in currently observed processes. We calculated radiative corrections due to NHL's to three such observables: leptonic widths of the $\mathrm{Z}$ boson $\Gamma_{l l}$, lepton universality breaking parameter $U_{b r}$ and the mass of the $\mathrm{W}$ boson $M_{W}$ (Chapters 6 and 7 ). We found that these observables form three complementary quantities as far as sensitivity to NHL masses and mixings is concerned. $\Gamma_{l l}$ depends on three kinds of radiative corrections: the vertex corrections (Sec. 6.4), the $\mathrm{Z}$ oblique corrections and the $\mathrm{W}$ oblique corrections (Sec. 6.3); the universality breaking parameter depends only on the vertex corrections; the $\mathrm{W}$ mass $M_{W}$ depends to a large degree only on the $\mathrm{W}$ oblique corrections.

The effect of the NHL mass $M_{N}$ in radiative corrections is, on the one hand, suppressed by the small mixings; on the other hand it is enhanced due to the violation of the Appelquist-Carazzone theorem (Sec. 6.6). These competing tendencies are reflected by the typical behaviour of the dominant terms in radiative corrections due to NHL's (see Eqs. 6.30, 6.53),

$$
\sim k_{H H} \frac{M_{N}^{2}}{M_{W}^{2}} \sim\left(\tau \tau_{m i x}\right)^{2} \frac{M_{N}^{2}}{M_{W}^{2}}
$$

To make up for the small mixings, only NHL's with masses in the TeV range can lead to significant deviations from the SM.

Assuming $\tau \tau_{\text {mix }}=0.033$, $e e_{\text {mix }}=\mu \mu_{\text {mix }}=0$ and $m_{t}=176 \mathrm{GeV}$, we derived the following limit on the NHL mass at the $2 \sigma$ level from the $Z$ decay width to $\tau$ leptons:

$$
M_{N} \leq 4.3 \mathrm{TeV}
$$


The universality breaking ratio, $U_{b r}$, leads to a yet better upper limit,

$$
M_{N} \leq 3.8 \mathrm{TeV}
$$

at the $2 \sigma$ level. We can use Eq. 8.1 to display the approximate dependence of the above limits on $\tau \tau_{m i x}$ :

$$
M_{N}<4.3 \times \frac{0.033}{\tau \tau_{m i x}} \mathrm{TeV}
$$

from Eq. 8.2 and

$$
M_{N}<3.8 \times \frac{0.033}{\tau \tau_{\operatorname{mix}}} \mathrm{TeV}
$$

from Eq. 8.3. Note the limits of Eqs. 8.2, 8.3 are comparable to the limit

$$
M_{N}<4 \mathrm{TeV}
$$

derived from the considerations of perturbation theory breakdown in Sec. 6.7.

We also found some sensitivity of the W mass $M_{W}$ to the NHL mass and mixings, which depends to a large degree on the top quark mass (Figs. 6.17a,b).

In Chapter 7 we generalized our analysis of Chapter 6 by relaxing the restriction $e e_{m i x}=\mu \mu_{m i x}=0$. We found that while the numerical results of Chapter 6 remain basically valid, they can be improved by the inclusion of the tree-level correction to the muon decay, $\left(1-\frac{1}{2} e e_{m i x}-\frac{1}{2} \mu \mu_{m i x}\right)$.

As already noted in Chapter 6, we feel there are at least two reasons which give the (flavour-conserving) processes studied in this thesis a distinct advantage over the flavour-violating ones. First, the limits on $M_{N}$ which we derived are only matched by those from $\mu \rightarrow$ eee. The flavour-violating decay rates for $\tau$ and $Z$ are below the current experimental sensitivity (see Sec. 5.1.3 and 5.2). Moreover, the 
$\mu \rightarrow$ eee decay depends only on $e e_{m i x}, e \mu_{m i x}$, the two of the six mixing parameters (see Eq. 5.30), and may be unobservable if $e e_{m i x}$ or $e \mu_{m i x}$ are very small. Second, the inequality Eq. 3.27 can further suppress the flavour-violating processes against the flavour-conserving ones via the 'conspiracy of the phases' in the sum of complex terms making up the flavour-violating parameters.

For these two reasons, first signatures of neutral heavy leptons could come from flavour-conserving observables. At this time, LEP has stopped its runs at the Z-peak energy and is running at $130-140 \mathrm{GeV}$. It will eventually be producing W pairs which will allow the mass $M_{W}$ to be measured with a precision of $0.05 \mathrm{GeV} 63$ (currently $M_{W}=80.410 \pm 0.180$ [53]). Combined with more precise measurements of the top quark mass we might be in a position to place even more stringent limits on NHL masses and mixings from our prediction of $M_{W}$ (see Figs. 6.17a,b).

The observation of neutral heavy leptons is essential for our understanding of the small neutrino masses. It would provide us with significant hints on grand unified theories and possibly superstring theories. 


\section{Appendix A}

\section{Dirac algebra and trace theorems}

In this Appendix we define the Dirac gamma matrices and collect their properties. We also show spin sums, some Fierz identities and the proof of the identity $\overline{\nu_{L}^{c}} \nu_{R}^{c}=$ $\overline{\nu_{L}} \nu_{R}$.

\section{A.1 Gamma matrices}

The so-called Dirac representation of the gamma matrices is given by

$$
\gamma^{0}=\left(\begin{array}{rr}
\mathbf{I} & 0 \\
0 & -\mathbf{I}
\end{array}\right), \quad \gamma^{i}=\left(\begin{array}{rr}
0 & \sigma^{i} \\
-\sigma^{i} & 0
\end{array}\right), \quad \gamma_{5} \equiv i \gamma^{0} \gamma^{1} \gamma^{2} \gamma^{3}=\left(\begin{array}{ll}
0 & \mathbf{I} \\
\mathbf{I} & 0
\end{array}\right),
$$

where $\mathbf{I}$ is $2 \times 2$ identity matrix and the Pauli matrices $\sigma^{i}$ are defined as

$$
\sigma^{1}=\left(\begin{array}{ll}
0 & 1 \\
1 & 0
\end{array}\right), \sigma^{2}=\left(\begin{array}{rr}
0 & -i \\
i & 0
\end{array}\right), \sigma^{3}=\left(\begin{array}{rr}
1 & 0 \\
0 & -1
\end{array}\right)
$$

Some properties of the gamma matrices follow:

$$
\gamma_{0}=\gamma^{0}, \quad \gamma_{i}=-\gamma^{i},
$$




$$
\begin{aligned}
\gamma_{0}^{\dagger} & =\gamma_{0}, \\
\gamma_{0} \gamma_{0} & =\mathbf{1}, \\
\gamma_{0} \gamma_{\mu}^{\dagger} \gamma_{0} & =\gamma_{\mu}, \\
\gamma_{0}^{T} & =\gamma_{0}, \\
\gamma_{5}^{T} & =\gamma_{5}, \\
\gamma_{5}^{2} & =\mathbf{1}, \\
\nu_{1}^{T} \Gamma \nu_{2} & =-\nu_{2}^{T} \Gamma^{T} \nu_{1},
\end{aligned}
$$

where $\nu_{1}, \nu_{2}$ are spinors, 1 is $4 \times 4$ unit matrix, and $\Gamma$ represents product of gamma matrices.

Trace theorems:

$$
\begin{aligned}
\operatorname{Tr}(\mathbf{1}) & =4, \\
\operatorname{Tr}\left(\gamma^{\mu} \gamma^{\nu}\right) & =4 g^{\mu \nu}, \\
\operatorname{Tr}\left(\gamma^{\mu} \gamma^{\nu} \gamma^{\lambda} \gamma^{\sigma}\right) & =4\left(g^{\mu \nu} g^{\lambda \sigma}-g^{\mu \lambda} g^{\nu \sigma}+g^{\mu \sigma} g^{\nu \lambda}\right), \\
\operatorname{Tr}\left(\gamma_{5}\right) & =0, \\
\operatorname{Tr}\left(\gamma_{5} \gamma^{\mu} \gamma^{\nu}\right) & =0, \\
\operatorname{Tr}\left(\gamma_{5} \gamma^{\mu} \gamma^{\nu} \gamma^{\lambda} \gamma^{\sigma}\right) & =4 i \epsilon^{\mu \nu \lambda \sigma}, \\
\operatorname{Tr}(\text { odd number of gamma matrices }) & =0 .
\end{aligned}
$$

Product rules:

$$
\begin{aligned}
\gamma^{\mu} \gamma^{\nu}+\gamma^{\nu} \gamma^{\mu} & =2 g^{\mu \nu} \\
\gamma_{\mu} \gamma^{\mu} & =4 \\
\gamma_{\mu} \gamma^{\nu} \gamma^{\mu} & =-2 \gamma^{\nu} \\
\gamma_{\mu} \gamma^{\nu} \gamma^{\lambda} \gamma^{\mu} & =4 g^{\nu \lambda}
\end{aligned}
$$




$$
\begin{aligned}
\gamma_{\mu} \gamma^{\nu} \gamma^{\lambda} \gamma^{\sigma} \gamma^{\mu} & =-2 \gamma^{\sigma} \gamma^{\lambda} \gamma^{\nu} \\
\gamma_{5} \gamma^{\mu}+\gamma^{\mu} \gamma_{5} & =0 \\
g^{\mu \nu} g_{\mu \nu} & =4
\end{aligned}
$$

An explicit representation of the charge conjugation matrix $\mathrm{C}$ is

$$
C=i \gamma^{2} \gamma^{0}=\left(\begin{array}{rrrr}
0 & 0 & 0 & -1 \\
0 & 0 & 1 & 0 \\
0 & -1 & 0 & 0 \\
1 & 0 & 0 & 0
\end{array}\right), \quad C^{T} C^{T}=-\mathbf{1}
$$

\section{A.2 Spin sums}

$$
\begin{aligned}
\sum_{s} u_{\alpha}(p, s) \overline{u_{\beta}}(p, s) & =(\not p+m)_{\alpha \beta}, \\
\sum_{s} v_{\alpha}(p, s) \overline{v_{\beta}}(p, s) & =(\not p-m)_{\alpha \beta}, \\
\sum_{\lambda} \epsilon_{\mu}(p, \lambda) \epsilon_{\nu}^{*}(p, \lambda) & =-g_{\mu \nu}+\frac{p_{\mu} p_{\nu}}{M_{V}^{2}},
\end{aligned}
$$

where $u_{\alpha}(p, s), v_{\alpha}(p, s)$ are spinors with momentum $p$, spin $s$ and mass $m ; \epsilon_{\mu}(p, \lambda)$ is a polarization vector of a weak boson $V=W, Z$ with momentum $p$, spin $\lambda$ and mass $M_{V}$.

\section{A.3 Fierz identities used for the calculation of the boxes}

$$
\begin{aligned}
{\left[\bar{u} \gamma_{\alpha}\left(1-\gamma_{5}\right) \gamma_{\epsilon} \gamma_{\gamma} u\right] \times\left[\bar{v}_{e} \gamma^{\alpha}\left(1-\gamma_{5}\right) \gamma^{\epsilon} \gamma^{\gamma} v_{\nu_{e}}\right] } & =16\left[\bar{u} \gamma_{\mu}\left(1-\gamma_{5}\right) u\right] \\
& \times\left[\bar{v}_{e} \gamma^{\mu}\left(1-\gamma_{5}\right) v_{\nu_{e}}\right]
\end{aligned}
$$




$$
\begin{aligned}
{\left[\bar{u} \gamma_{\alpha}\left(1-\gamma_{5}\right) \gamma_{\epsilon} \gamma_{\gamma} u\right] \times\left[\bar{v}_{e} \gamma^{\gamma}\left(1-\gamma_{5}\right) \gamma^{\epsilon} \gamma_{\alpha} v_{\nu_{e}}\right] } & =4\left[\bar{u} \gamma_{\mu}\left(1-\gamma_{5}\right) u\right] \\
& \times\left[\bar{v}_{e} \gamma^{\mu}\left(1-\gamma_{5}\right) v_{\nu_{e}}\right]
\end{aligned}
$$

\section{A.4 Proof of the identity $\overline{\nu_{L}^{c}} \nu_{R}^{c}=\overline{\nu_{L}} \nu_{R}$}

Using the definition of the charge conjugate field,

$$
\begin{aligned}
& \nu^{c}=C \gamma_{0} \nu^{*}, \quad\left(C=i \gamma^{2} \gamma^{0}\right) \\
& \overline{\nu^{c}}=\nu^{T} C,
\end{aligned}
$$

we get

$$
\begin{aligned}
\overline{\nu_{L}^{c}} \nu_{R}^{c} & =\left[\frac{1-\gamma_{5}}{2} \nu^{c}\right]^{\dagger} \gamma_{0} \frac{1+\gamma_{5}}{2} \nu^{c}=\nu^{c \dagger} \frac{1-\gamma_{5}^{\dagger}}{2} \gamma_{0} \frac{1+\gamma_{5}}{2} \nu^{c} \\
& =\nu^{c \dagger} \gamma_{0} \frac{1+\gamma_{5}}{2} \frac{1+\gamma_{5}}{2} \nu^{c}=\overline{\nu^{c}} \frac{1+\gamma_{5}}{2} \nu^{c} \\
& =\nu^{T} C \frac{1+\gamma_{5}}{2} C \gamma_{0} \nu^{*}=-\nu^{* T}\left[C \frac{1+\gamma_{5}}{2} C \gamma_{0}\right]^{T} \nu \\
& =-\nu^{\dagger}\left[\gamma_{0} C^{T} \frac{1+\gamma_{5}}{2} C^{T}\right] \nu=-\bar{\nu} C^{T} \frac{1+\gamma_{5}}{2} C^{T} \nu \\
& =-\bar{\nu} C^{T} C^{T} \frac{1+\gamma_{5}}{2} \nu=\bar{\nu} \frac{1+\gamma_{5}}{2} \nu \\
& =\overline{\nu_{L}} \nu_{R} .
\end{aligned}
$$




\section{Appendix B}

\section{Couplings of $\nu^{\prime}$ and $N$ to Higgs}

We begin with some useful properties of the rotation matrix $G$ (see Eq. 3.12). From $G G^{\dagger}=G^{\dagger} G=1$ we have

$$
\begin{aligned}
& U_{1} U_{1}^{\dagger}+U_{2} U_{2}^{\dagger}=1, \quad U_{1}^{\dagger} U_{1}+U_{3}^{\dagger} U_{3}=1, \\
& U_{3} U_{3}^{\dagger}+U_{4} U_{4}^{\dagger}=1, \quad U_{2}^{\dagger} U_{2}+U_{4}^{\dagger} U_{4}=1 \text {, } \\
& U_{1} U_{3}^{\dagger}+U_{2} U_{4}^{\dagger}=0, \quad U_{1}^{\dagger} U_{2}+U_{3}^{\dagger} U_{4}=0, \\
& U_{3} U_{1}^{\dagger}+U_{4} U_{2}^{\dagger}=0, \quad U_{2}^{\dagger} U_{1}+U_{4}^{\dagger} U_{3}=0 .
\end{aligned}
$$

Further, from (see Sec. 3.1.2)

$$
\begin{aligned}
& U_{1} D+U_{2} M=0, \\
& U_{3} D+U_{4} M=M^{\prime},
\end{aligned}
$$

we get

$$
\begin{aligned}
U_{2}^{\dagger} U_{1} D+U_{2}^{\dagger} U_{2} M & =0, \\
U_{4}^{\dagger} U_{3} D+U_{4}^{\dagger} U_{4} M & =U_{4}^{\dagger} M^{\prime} .
\end{aligned}
$$


Adding these two equations and using Eq. B.1 we find the following relation between $M$ and $M^{\prime}$ :

$$
M=U_{4}^{\dagger} M^{\prime}
$$

To derive the Lagrangian describing the couplings of $\nu^{\prime}$ and $N$ to $H, \mathcal{L}_{H}$, we rewrite Eq. 3.30 as

$$
\mathcal{L}=-\frac{g_{2}}{\sqrt{2} M_{W}}\left(\overline{\nu_{L}} \overline{l_{L}}\right) D \tilde{\Phi} n_{R}+\text { h.c. }
$$

where $D$ is a $3 \times 3$ matrix in family space and, (see Eqs. 2.16, 2.23),

$$
\tilde{\Phi}=i \tau_{2} \Phi^{*}=\left(\begin{array}{c}
\phi^{0^{*}} \\
-\phi^{-}
\end{array}\right)=\left(\begin{array}{c}
\frac{1}{\sqrt{2}}(v+H-i \chi) \\
-\phi^{-}
\end{array}\right)
$$

Selecting the $H$ part we get

$$
\mathcal{L}_{H}=-\frac{g_{2}}{2 M_{W}} \overline{\nu_{L}} D n_{R} H+\text { h.c. }
$$

In the next step we add and subtract a term:

$$
\begin{aligned}
\mathcal{L}_{H} & =-\frac{g_{2}}{2 M_{W}} \overline{\nu_{L}} D n_{R} H+\text { h.c. } \\
& -\frac{g_{2}}{2 M_{W}} \overline{S_{L}} M n_{R} H+\text { h.c. } \\
& +\frac{g_{2}}{2 M_{W}} \overline{S_{L}} M n_{R} H+\text { h.c. }
\end{aligned}
$$

The first two lines of this relation can be compared with Eq. 3.10. We can now use the results of Sec. 3.1.2, which give

$$
\begin{aligned}
\mathcal{L}_{H} & =-\frac{g_{2}}{2 M_{W}} \overline{S_{L}^{\prime}} M^{\prime} n_{R} H+\text { h.c. } \\
& +\frac{g_{2}}{2 M_{W}}\left(\overline{\nu_{L}^{\prime}} U_{2}+\overline{S_{L}^{\prime}} U_{4}\right) M n_{R} H+\text { h.c. }
\end{aligned}
$$




$$
\begin{aligned}
& =-\frac{g_{2}}{2 M_{W}} \overline{S_{L}^{\prime \prime}} T M^{\prime} Z^{\dagger} n_{R}^{\prime \prime} H+\text { h.c. } \\
& +\frac{g_{2}}{2 M_{W}}\left(\overline{\nu_{L}^{\prime}} U_{2} M Z^{\dagger}+\overline{S_{L}^{\prime \prime}} T U_{4} M Z^{\dagger}\right) n_{R}^{\prime \prime} H+\text { h.c. }
\end{aligned}
$$

Now we use $M=U_{4}^{\dagger} M^{\prime}$ (see Eq. B.4) and $M^{\prime \prime}=T M^{\prime} Z^{\dagger}$ (see Eq. 3.17):

$$
\begin{aligned}
\mathcal{L}_{H} & =-\frac{g_{2}}{2 M_{W}} \overline{S_{L}^{\prime \prime}} T M^{\prime} Z^{\dagger} n_{R}^{\prime \prime} H+\text { h.c. } \\
& +\frac{g_{2}}{2 M_{W}}\left(\overline{\nu_{L}^{\prime}} U_{2} U_{4}^{\dagger} M^{\prime} Z^{\dagger}+\overline{S_{L}^{\prime \prime}} T U_{4} U_{4}^{\dagger} M^{\prime} Z^{\dagger}\right) n_{R}^{\prime \prime} H+\text { h.c. } \\
& =-\frac{g_{2}}{2 M_{W}} \overline{S_{L}^{\prime \prime}} M^{\prime \prime} n_{R}^{\prime \prime} H+h . c . \\
& +\frac{g_{2}}{2 M_{W}}\left(\overline{\nu_{L}^{\prime}} U_{2} U_{4}^{\dagger} T^{\dagger} T M^{\prime} Z^{\dagger}+\overline{S_{L}^{\prime \prime}} T U_{4} U_{4}^{\dagger} T^{\dagger} T M^{\prime} Z^{\dagger}\right) n_{R}^{\prime \prime} H+\text { h.c. } \\
& =-\frac{g_{2}}{2 M_{W}} \overline{S_{L}^{\prime \prime}} M^{\prime \prime} n_{R}^{\prime \prime} H+h . c . \\
& +\frac{g_{2}}{2 M_{W}}\left(\overline{\nu_{L}^{\prime}} U_{2} U_{4}^{\dagger} T^{\dagger} M^{\prime \prime}+\overline{S_{L}^{\prime \prime}} T U_{4} U_{4}^{\dagger} T^{\dagger} M^{\prime \prime}\right) n_{R}^{\prime \prime} H+\text { h.c. . }
\end{aligned}
$$

Using $U_{2} U_{4}^{\dagger}=-U_{1} U_{3}^{\dagger}$ and $U_{4} U_{4}^{\dagger}=1-U_{3} U_{3}^{\dagger}$, see Eq. B.1,

$$
\begin{aligned}
\mathcal{L}_{H} & =-\frac{g_{2}}{2 M_{W}} \overline{S_{L}^{\prime \prime}} M^{\prime \prime} n_{R}^{\prime \prime} H+\text { h.c. } \\
& +\frac{g_{2}}{2 M_{W}}\left[\overline{\nu_{L}^{\prime}}\left(-U_{1} U_{3}^{\dagger}\right) T^{\dagger} M^{\prime \prime}+\overline{S_{L}^{\prime \prime}} T\left(1-U_{3} U_{3}^{\dagger}\right) T^{\dagger} M^{\prime \prime}\right] n_{R}^{\prime \prime} H+\text { h.c. }
\end{aligned}
$$

and putting

$$
K_{L}=U_{1}^{\dagger}, \quad K_{H}=U_{3}^{\dagger} T^{\dagger}
$$

we get

$$
\begin{aligned}
\mathcal{L}_{H} & =-\frac{g_{2}}{2 M_{W}} \overline{S_{L}^{\prime \prime}} M^{\prime \prime} n_{R}^{\prime \prime} H+\text { h.c. } \\
& +\frac{g_{2}}{2 M_{W}} \overline{\nu_{L}^{\prime}}\left(-K_{L}^{\dagger} K_{H}\right) M^{\prime \prime} n_{R}^{\prime \prime} H+\text { h.c. } \\
& +\frac{g_{2}}{2 M_{W}} \overline{S_{L}^{\prime \prime}}\left(1-K_{H}^{\dagger} K_{H}\right) M^{\prime \prime} n_{R}^{\prime \prime} H+\text { h.c. }
\end{aligned}
$$




$$
\begin{aligned}
& =-\frac{g_{2}}{2 M_{W}} \overline{S_{L}^{\prime \prime}}\left(K_{H}^{\dagger} K_{H}\right) M^{\prime \prime} n_{R}^{\prime \prime} H+h . c . \\
& -\frac{g_{2}}{2 M_{W}} \overline{\nu_{L}^{\prime}}\left(K_{L}^{\dagger} K_{H}\right) M^{\prime \prime} n_{R}^{\prime \prime} H+h . c . \\
& =-\frac{g_{2}}{2 M_{W}} \overline{N_{L}}\left(K_{H}^{\dagger} K_{H}\right) M^{\prime \prime} N_{R} H+h . c . \\
& -\frac{g_{2}}{2 M_{W}} \overline{\nu_{L}^{\prime}}\left(K_{L}^{\dagger} K_{H}\right) M^{\prime \prime} N_{R} H+h . c . \\
& =-\frac{g_{2}}{2 M_{W}} \bar{N}\left(K_{H}^{\dagger} K_{H}\right) M_{N} N H \\
& -\frac{g_{2}}{2 M_{W}} \overline{\nu^{\prime}}\left(K_{L}^{\dagger} K_{H}\right) M_{N} \frac{1+\gamma_{5}}{2} N H \\
& -\frac{g_{2}}{2 M_{W}} \bar{N}\left(K_{H}^{\dagger} K_{L}\right) M_{N} \frac{1-\gamma_{5}}{2} \nu^{\prime} H .
\end{aligned}
$$

The couplings of $\chi, \phi^{+}$and $\phi^{-}$are found by analogy. 


\section{Appendix $\mathrm{C}$}

\section{Feynman rules}

We list here the Feynman rules needed for the computation of the non-SM diagrams contributing to the processes studied in this thesis. They are given in the 't Hooft-Feynman gauge (see Sec. 4.1). The rules for the vertices correspond to the interaction Lagrangians of Sec. 3.3. The SM case is obtained in the limit

$$
K_{H} \rightarrow 0, \quad K_{L} \rightarrow 1 .
$$

In vertices, where applicable, the arrows indicate in addition to the flow of the charge also the flow of momenta. 


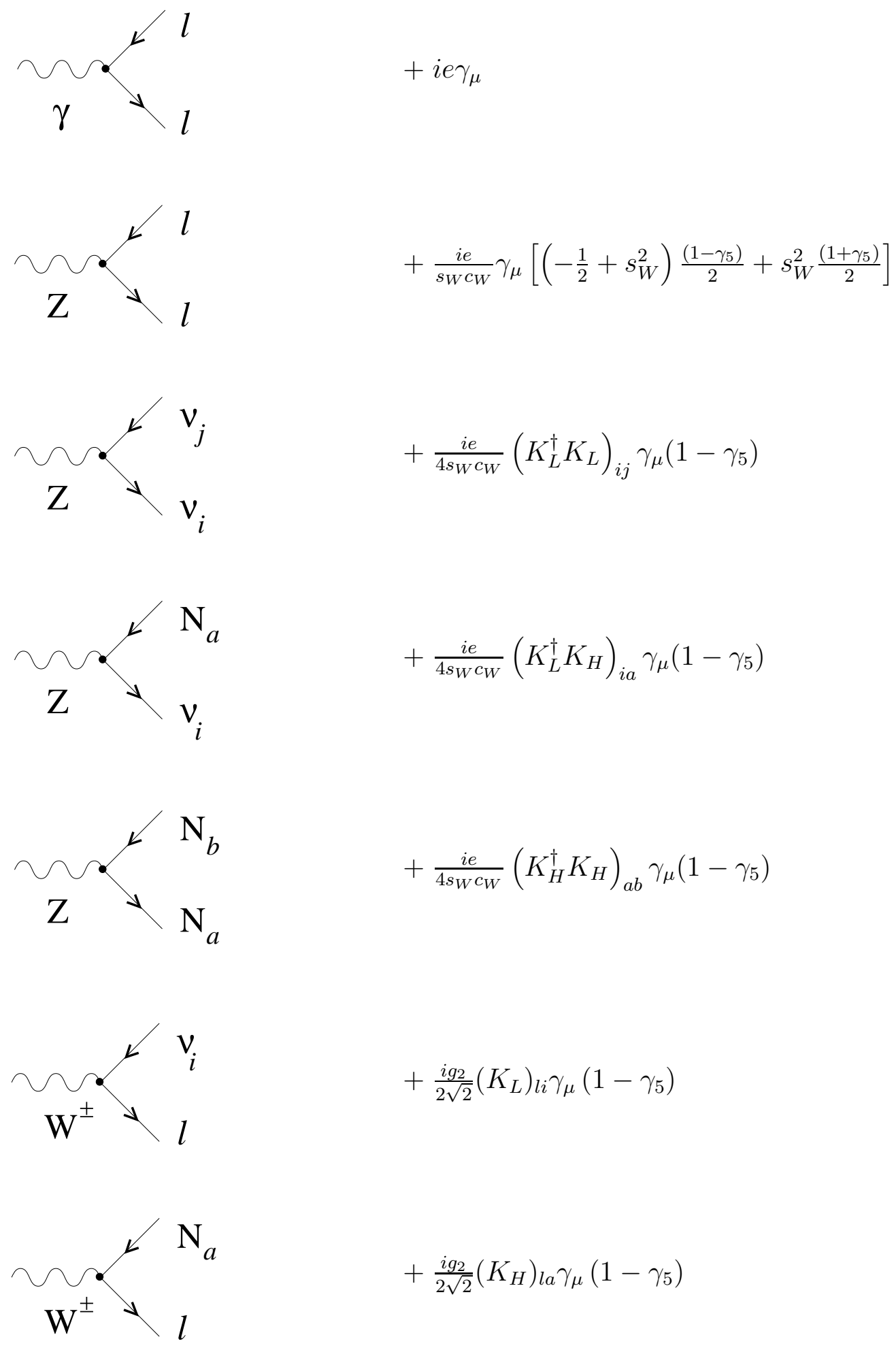




$$
\begin{aligned}
& \begin{array}{l}
\nearrow \mathrm{v}_{i} \\
\mathrm{H} \mathrm{N}_{a}
\end{array} \\
& -\frac{i g_{2}}{2 M_{W}} M_{N}\left(K_{L}^{\dagger} K_{H}\right)_{i a} \frac{\left(1+\gamma_{5}\right)}{2} \\
& \mathrm{H} r \mathrm{~N}_{a} \\
& -\frac{i g_{2}}{2 M_{W}} M_{N}\left(K_{H}^{\dagger} K_{L}\right)_{a i} \frac{\left(1-\gamma_{5}\right)}{2} \\
& x+\mathrm{v}_{i} \\
& -\frac{g_{2}}{2 M_{W}} M_{N}\left(K_{L}^{\dagger} K_{H}\right)_{i a} \frac{\left(1+\gamma_{5}\right)}{2} \\
& x<v_{i} \\
& +\frac{g_{2}}{2 M_{W}} M_{N}\left(K_{H}^{\dagger} K_{L}\right)_{a i} \frac{\left(1-\gamma_{5}\right)}{2} \\
& \mathrm{H} r \mathrm{~N}_{a} \\
& -\frac{i g_{2}}{2 M_{W}} M_{N}\left(K_{H}^{\dagger} K_{H}\right)_{a b} \\
& \chi \uparrow \mathrm{N}_{b} \\
& -\frac{g_{2}}{2 M_{W}} M_{N}\left(K_{H}^{\dagger} K_{H}\right)_{a b} \gamma_{5}
\end{aligned}
$$




$$
\begin{aligned}
& \phi^{-} \mathrm{N}_{a} \\
& +\frac{i g_{2}}{\sqrt{2} M_{W}}\left(K_{H}\right)_{l a}\left[M_{N} \frac{\left(1+\gamma_{5}\right)}{2}-m_{l} \frac{\left(1-\gamma_{5}\right)}{2}\right] \\
& \phi^{-} r v_{i} \\
& \phi^{+} \mathrm{N}_{a} \\
& +\frac{i g_{2}}{\sqrt{2} M_{W}}\left(K_{H}^{\dagger}\right)_{a l}\left[M_{N} \frac{\left(1-\gamma_{5}\right)}{2}-m_{l} \frac{\left(1+\gamma_{5}\right)}{2}\right] \\
& \phi^{+} \uparrow_{l} v_{i} \\
& -\frac{i g_{2} m_{l}}{\sqrt{2} M_{W}}\left(K_{L}^{\dagger}\right)_{i l} \frac{\left(1+\gamma_{5}\right)}{2} \\
& \mathrm{Z}_{\mu}\left(\mathrm{p}_{3}\right)^{\lambda} \mathrm{W}_{\lambda}^{-}\left(\mathrm{p}_{2}\right) \\
& -\frac{i e c_{W}}{s_{W}}\left[g_{\nu \lambda}\left(p_{1}-p_{2}\right)_{\mu}+g_{\lambda \mu}\left(p_{2}-p_{3}\right)_{\nu}\right. \\
& \left.+g_{\mu \nu}\left(p_{3}-p_{1}\right)_{\lambda}\right] \\
& \sum_{\mathrm{W}_{\mu}} \mathrm{W}_{v} \\
& +i g_{2} M_{W} g^{\mu \nu}
\end{aligned}
$$




$$
\begin{aligned}
& \chi \sum_{2} \mathrm{~W}_{v} \\
& \begin{array}{lll}
\mathrm{W}_{\mu}^{+} & \phi^{-}\left(\mathrm{p}_{-}\right) \\
& \mathrm{H}\left(\mathrm{p}_{1}\right)
\end{array} \\
& \mathrm{W}_{\mu}^{+} \phi^{\phi^{-}\left(\mathrm{p}_{-}\right)}
\end{aligned}
$$

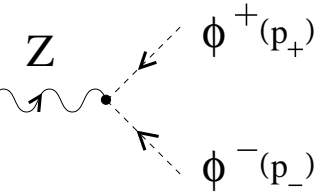

$$
\begin{aligned}
& \phi^{+} \sum_{\mathrm{W}_{\mu}^{-}}^{\mathrm{Z}_{v}} \\
& 0 \\
& +\frac{i g_{2}}{2}\left(p_{1}-p_{-}\right)_{\mu} \\
& +\frac{g_{2}}{2}\left(p_{-}-p_{2}\right)_{\mu} \\
& -\frac{i g_{2}}{2} \frac{1-2 s_{W}^{2}}{c_{W}}\left(p_{-}-p_{+}\right)_{\mu} \\
& -i g_{2} M_{W} \frac{s_{W}^{2}}{c_{W}} g^{\mu \nu} \\
& -i g_{2} M_{W} \frac{s_{W}^{2}}{c_{W}} g^{\mu \nu} \\
& \phi^{-} \mathcal{Z}_{\mathrm{W}_{\mu}} \mathrm{Z}_{v}
\end{aligned}
$$




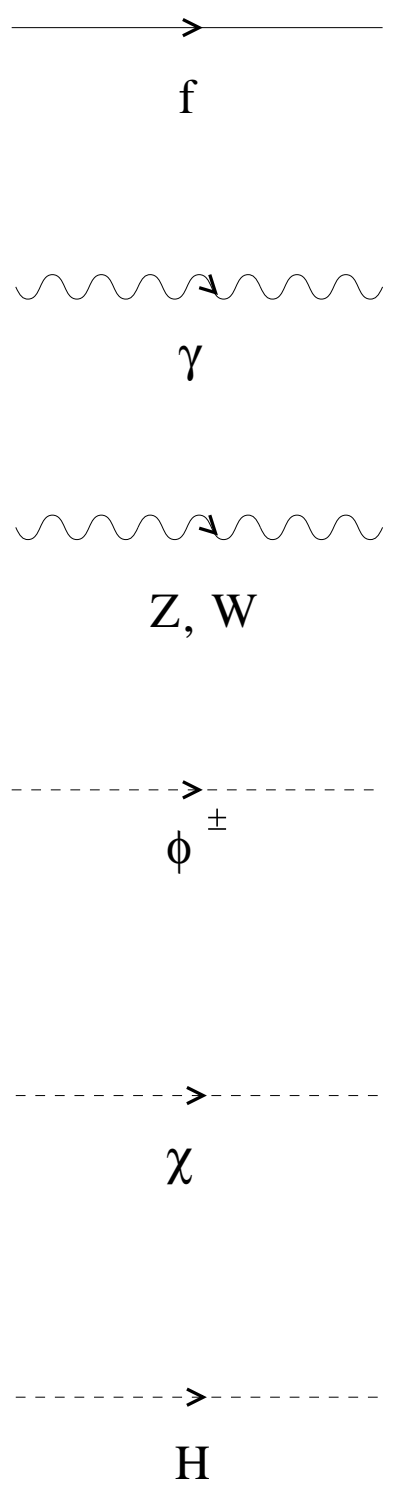

$+\frac{i}{q-m}$

$-\frac{i g^{\alpha \beta}}{q^{2}}$

$-\frac{i g^{\alpha \beta}}{q^{2}-M_{V}^{2}}$

$+\frac{i}{q^{2}-M_{W}^{2}}$

$+\frac{i}{q^{2}-M_{Z}^{2}}$

$+\frac{i}{q^{2}-M_{H}^{2}}$ 


\section{Appendix D}

\section{Dimensional regularization and some useful integrals}

\section{D.1 Dimensional regularization}

Before one-loop amplitudes with divergent momentum integrals can be renormalized, they have to be regularized. Regularization defines integrals, parametrizes their divergences, and separates their finite parts. Dimensional regularization [40] defines integrals by analytically continuing them from 4 -dimensional to $n$-dimensional spacetime. The computation of integrals in $n$ dimensions typically yields (see Eq. 4.9)

$$
\Lambda_{V}(0)=-\frac{\alpha_{137}}{4 \pi}\left(\frac{2}{\epsilon}+\text { finite constants }\right), \quad \epsilon=4-n,
$$

that is, the divergence is parametrized as a simple pole at $n=4$. In our calculations, we used the following momentum integrals in $n$ dimensions:

$$
I_{0}(l)=\int \frac{d^{n} k}{(2 \pi)^{n}} \frac{1}{\left(k^{2}+2 k \cdot s+t\right)^{l}}=\frac{i(-\pi)^{n / 2}}{(2 \pi)^{n}} \frac{\Gamma(l-n / 2)}{\Gamma(l)} \frac{1}{\left(t-s^{2}\right)^{(l-n / 2)}}
$$




$$
\begin{aligned}
& \equiv N(l) \frac{1}{\left(t-s^{2}\right)^{(l-n / 2)}}, \\
I_{\mu}(l) & =\int \frac{d^{n} k}{(2 \pi)^{n}} \frac{k_{\mu}}{\left(k^{2}+2 k \cdot s+t\right)^{l}}=-s_{\mu} I_{0}(l), \\
I_{\mu \nu}(l) & =\int \frac{d^{n} k}{(2 \pi)^{n}} \frac{k_{\mu} k_{\nu}}{\left(k^{2}+2 k \cdot s+t\right)^{l}}=I_{0}(l)\left[s_{\mu} s_{\nu}+\frac{1}{2} g_{\mu \nu}\left(t-s^{2}\right)\right. \\
& \left.\times \frac{1}{l-n / 2-1}\right] .
\end{aligned}
$$

Specifically, for $l=2,3$ we have

$$
\begin{aligned}
& N(2)=\frac{i(-\pi)^{\frac{n}{2}}}{(2 \pi)^{n}} \frac{\Gamma\left(2-\frac{n}{2}\right)}{\Gamma(2)}=\frac{i(-1)^{\frac{n}{2}}}{(4 \pi)^{2}}\left(1+\frac{\epsilon}{2} \ln 4 \pi\right)\left(\frac{2}{\epsilon}-\gamma\right), \\
& N(3)=\frac{i(-\pi)^{\frac{n}{2}}}{(2 \pi)^{n}} \frac{\Gamma\left(3-\frac{n}{2}\right)}{\Gamma(3)}=\frac{i(-1)^{\frac{n}{2}}}{2(4 \pi)^{2}}\left(1+\frac{\epsilon}{2} \ln 4 \pi\right)\left(1-\frac{\epsilon}{2} \gamma\right),
\end{aligned}
$$

where we used

$$
\begin{gathered}
\Gamma\left(2-\frac{n}{2}\right)=\Gamma\left(2-\frac{4-\epsilon}{2}\right)=\Gamma\left(\frac{\epsilon}{2}\right)=\frac{2}{\epsilon}-\gamma \\
\Gamma\left(3-\frac{n}{2}\right)=\Gamma\left(1+\frac{\epsilon}{2}\right)=\frac{\epsilon}{2} \Gamma\left(\frac{\epsilon}{2}\right)=1-\frac{\epsilon}{2} \gamma
\end{gathered}
$$

where $\gamma \doteq 0.5772$ is Euler-Mascheroni constant. In $n=4-\epsilon$ dimensions, $\alpha$ becomes a dimensional quantity:

$$
\alpha=\frac{e^{2}}{4 \pi} \rightarrow \alpha \mu^{\epsilon}=\alpha\left(1+\frac{\epsilon}{2} \ln \mu^{2}+\ldots\right)
$$

where $\mu$ is an arbitrary mass scale. The combination of Eq. D.4 and Eq. D.7 yields

$$
\alpha \mu^{\epsilon}\left(1+\frac{\epsilon}{2} \ln 4 \pi\right)\left(\frac{2}{\epsilon}-\gamma\right)=\alpha\left(\frac{2}{\epsilon}-\gamma+\ln 4 \pi+\ln \mu^{2}\right)=\alpha \Delta_{\mu} .
$$

It is this $\Delta_{\mu}$ rather than $\frac{2}{\epsilon}$, which is usually thought of as the parameterization of the divergence since factors $\gamma, \ln 4 \pi$ and $\ln \mu^{2}$ are always present along with $\frac{2}{\epsilon}$ and 
they all together cancel out in renormalized quantities. We use also other variants of the $\Delta$ symbol:

$$
\begin{aligned}
\Delta & =\frac{2}{4-n}-\gamma-\ln \pi=\frac{2}{\epsilon}-\gamma-\ln \pi, \\
\Delta_{\mu} & =\frac{2}{\epsilon}-\gamma+\ln 4 \pi+\ln \mu^{2}, \\
\Delta_{m} & =\frac{2}{\epsilon}-\gamma+\ln 4 \pi-\ln \frac{m^{2}}{\mu^{2}} .
\end{aligned}
$$

To cast momentum integrals into the form of Eq. D.1, the following Feynman parameterization is used:

$$
\begin{aligned}
\frac{1}{a_{0} a_{1} a_{2} \ldots a_{n}} & =\Gamma(n+1) \int_{0}^{1} d x_{1} \int_{0}^{x_{1}} d x_{2} \ldots \int_{0}^{x_{n-1}} d x_{n} \\
& \times \frac{1}{\left[a_{0}+\left(a_{1}-a_{0}\right) x_{1}+\ldots\left(a_{n}-a_{n-1}\right) x_{n}\right]^{n+1}} .
\end{aligned}
$$

Higher powers in $a_{i}$ are obtained by differentiation with respect to this parameter. In this work, these specific expressions were used:

$$
\begin{aligned}
\frac{1}{a b} & =\int_{0}^{1} d x \frac{1}{[a+(b-a) x]^{2}} \\
\frac{1}{a b c} & =2 \int_{0}^{1} d x \int_{0}^{x} d y \frac{1}{[a+(b-a) x+(c-b) y]^{3}} \\
\frac{1}{a b c} & =2 \int_{0}^{1} d x \int_{0}^{1} d y \frac{y}{[a x y+b y(1-x)+c(1-y)]^{3}} \\
\frac{1}{a b c^{2}} & =6 \int_{0}^{1} d x \int_{0}^{x} d y \frac{y}{[a+(b-a) x+(c-b) y]^{4}} .
\end{aligned}
$$

In $n$ dimensions, the algebra of the Dirac matrices described in Appendix A is generalized as follows:

$$
\begin{aligned}
\gamma^{\mu} \gamma^{\nu}+\gamma^{\nu} \gamma^{\mu} & =2 g^{\mu \nu} \\
g^{\mu \nu} g_{\mu \nu} & =n
\end{aligned}
$$




$$
\begin{aligned}
\gamma_{\mu} \gamma^{\mu} & =n \\
\operatorname{Tr}\left(\gamma^{\mu} \gamma^{\nu}\right) & =n g^{\mu \nu} \\
\operatorname{Tr}\left(\gamma^{\mu} \gamma^{\nu} \gamma^{\lambda} \gamma^{\sigma}\right) & =n\left(g^{\mu \nu} g^{\lambda \sigma}-g^{\mu \lambda} g^{\nu \sigma}+g^{\mu \sigma} g^{\nu \lambda}\right) \\
\gamma_{\mu} \gamma^{\nu} \gamma^{\mu} & =(2-n) \gamma^{\nu} \\
\gamma_{\mu} \gamma^{\nu} \gamma^{\lambda} \gamma^{\mu} & =4 g^{\nu \lambda}+(n-4) \gamma^{\nu} \gamma^{\lambda}, \\
\gamma_{\mu} \gamma^{\nu} \gamma^{\lambda} \gamma^{\sigma} \gamma^{\mu} & =-2 \gamma^{\sigma} \gamma^{\lambda} \gamma^{\nu}-(n-4) \gamma^{\nu} \gamma^{\lambda} \gamma^{\sigma}, \\
\gamma_{5} \gamma^{\mu}+\gamma^{\mu} \gamma_{5} & =0 .
\end{aligned}
$$

\section{D.2 The computation of $\mathcal{I}_{0}, \mathcal{I}_{1}(m), \mathcal{I}_{2}(m)$ and $\mathcal{I}_{3}(m)$ integrals}

We start with two useful integrals one often encounters during the computation of momentum integrals:

$$
\begin{aligned}
\int_{0}^{1} d x \ln (C x+D) & =\ln (C+D)+\frac{D}{C} \ln \frac{C+D}{D}-1 \\
\int_{0}^{1} d x[x \ln (E x+F)] & =\frac{(E+F)^{2}}{2 E^{2}} \ln (E+F)-\frac{F^{2}}{2 E^{2}} \ln F-\frac{1}{2 E^{2}} \frac{(E+F)^{2}}{2} \\
& +\frac{1}{4} \frac{F^{2}}{E^{2}}-\frac{F(E+F)}{E^{2}} \ln (E+F)+\frac{F E}{E^{2}} \\
& +\frac{F^{2} \ln F}{E^{2}} .
\end{aligned}
$$

To compute $\mathcal{I}_{0}, \mathcal{I}_{1}(m), \mathcal{I}_{2}(m)$ and $\mathcal{I}_{3}(m)$ we note these integrals (defined in Sec. 7.1) are related via

$$
\mathcal{I}_{2}\left(M_{Z}\right)=\mathcal{I}_{1}+M_{N}^{2} \mathcal{I}_{3}\left(M_{Z}\right)
$$

and the integral $\mathcal{I}_{0}$ is obtained as a special case of $\mathcal{I}_{1}(m)$ for $M_{N} \rightarrow 0$. To calculate 
the $\mathcal{I}_{1}(m)$ integral, we use the parameterization of Eq. D.13:

$$
\frac{1}{a b c}=2 \int_{0}^{1} d x \int_{0}^{1} d y \frac{y}{[a x y+b y(1-x)+c(1-y)]^{3}}
$$

The term in the denominator is

$$
\begin{aligned}
a x y+b y(1-x)+c(1-y) & =\left(k^{2}-M_{W}^{2}\right) x y+\left(k^{2}-m^{2}\right)(y-x y) \\
& +\left(k^{2}-M_{N}^{2}\right)(1-y) \\
& =k^{2}-M_{W}^{2} x y+m^{2} x y-m^{2} y \\
& -M_{N}^{2}(1-y) .
\end{aligned}
$$

Using the momentum integral Eq. D.1, we can write for $\mathcal{I}_{1}(m)$

$$
\begin{aligned}
\frac{i}{(4 \pi)^{2}} \mathcal{I}_{1}(m) & =\int \frac{d^{4} k}{(2 \pi)^{4}} \frac{1}{\left(k^{2}-M_{N}^{2}\right)\left(k^{2}-M_{W}^{2}\right)\left(k^{2}-m^{2}\right)} \\
& =2 \int_{0}^{1} d x \int_{0}^{1} d y \int \frac{d^{4} k}{(2 \pi)^{4}} \\
& \times \frac{y}{\left[k^{2}-M_{W}^{2} x y+m^{2} x y-m^{2} y-M_{N}^{2}(1-y)\right]^{3}} \\
& =\frac{i}{(4 \pi)^{2}} \int_{0}^{1} d x \int_{0}^{1} d y \\
& \times \frac{y}{\left(m^{2}-M_{W}^{2}\right) y x-m^{2} y-M_{N}^{2}(1-y)} .
\end{aligned}
$$

First we integrate over the $x$ parameter:

$$
\begin{aligned}
\mathcal{I}_{1}(m) & =\int_{0}^{1} d y y \int_{0}^{1} d x \frac{-1}{\left(M_{W}^{2}-m^{2}\right) y x+m^{2} y+M_{N}^{2}(1-y)} \\
& =\int_{0}^{1} d y y \int_{0}^{1} d x \frac{-1}{A x+B}=-\int_{0}^{1} d y y \frac{1}{A}[\ln (A+B)-\ln B] \\
& =\int_{0}^{1} d y y \frac{1}{\left(m^{2}-M_{W}^{2}\right) y}\left[\ln \left(M_{W}^{2} y+M_{N}^{2}-M_{N}^{2} y\right)\right. \\
& \left.-\ln \left(m^{2} y+M_{N}^{2}-M_{N}^{2} y\right)\right],
\end{aligned}
$$


and then the integration over $y$ :

$$
\begin{aligned}
\mathcal{I}_{1}(m) & =\int_{0}^{1} d y \frac{1}{\left(m^{2}-M_{W}^{2}\right)}\left[\ln \left(M_{W}^{2} y+M_{N}^{2}-M_{N}^{2} y\right)-\ln \left(m^{2} y+M_{N}^{2}-M_{N}^{2} y\right)\right] \\
& =\frac{1}{\left(m^{2}-M_{W}^{2}\right)}\left[\int_{0}^{1} d y \ln (C y+D)-\int_{0}^{1} d y \ln (E y+F)\right] \\
& =\frac{1}{\left(m^{2}-M_{W}^{2}\right)}\left[\ln (C+D)+\frac{D}{C} \ln \frac{C+D}{D}-1-\ln (E+F)\right. \\
& \left.-\frac{F}{E} \ln \frac{E+F}{F}+1\right] \\
& =\frac{1}{\left(m^{2}-M_{W}^{2}\right)}\left[\ln \frac{M_{W}^{2}}{m^{2}}+\frac{M_{N}^{2}}{M_{W}^{2}-M_{N}^{2}} \ln \frac{M_{W}^{2}}{M_{N}^{2}}-\frac{M_{N}^{2}}{m^{2}-M_{N}^{2}} \ln \frac{m^{2}}{M_{N}^{2}}\right] .(\mathrm{D} \cdot 21)
\end{aligned}
$$

The $\mathcal{I}_{3}(m)$ integral is found using similar steps:

$$
\begin{aligned}
\mathcal{I}_{3}(m) & =\frac{(4 \pi)^{2}}{i} \int \frac{d^{4} k}{(2 \pi)^{4}} \frac{1}{\left(k^{2}-M_{N}^{2}\right)^{2}\left(k^{2}-M_{W}^{2}\right)\left(k^{2}-m^{2}\right)} \\
& =6 \int \frac{d^{4} k}{(2 \pi)^{4}} \int_{0}^{1} d x \int_{0}^{x} d y \frac{y}{\left[k^{2}-M_{W}^{2}+\left(M_{W}^{2}-m^{2}\right) x+\left(m^{2}-M_{N}^{2}\right) y\right]^{4}} \\
& =\int_{0}^{1} d x \int_{0}^{x} d y \frac{y}{\left[\left(M_{W}^{2}-m^{2}\right) x-M_{W}^{2}+\left(m^{2}-M_{N}^{2}\right) y\right]^{2}} \\
& =\frac{1}{m^{2}-M_{W}^{2}}\left\{\frac{1}{M_{N}^{2}-M_{W}^{2}}+\frac{M_{W}^{2} \ln \frac{M_{W}^{2}}{M_{N}^{2}}}{\left(M_{N}^{2}-M_{W}^{2}\right)^{2}}-\frac{1}{M_{N}^{2}-m^{2}}\right. \\
& \left.-\frac{m^{2} \ln \frac{m^{2}}{M_{N}^{2}}}{\left(M_{N}^{2}-m^{2}\right)^{2}}\right\} .
\end{aligned}
$$




\section{Appendix E}

\section{Renormalization constants,}

unrenormalized self-energies and 't Hooft scalar integrals

\section{E.1 Renormalization constants}

It is convenient to define the following linear combinations of the renormalization constants $(i=1,2)$ :

$$
\begin{aligned}
\delta Z_{i}^{\gamma} & =s_{W}^{2} \delta Z_{i}^{W}+c_{W}^{2} \delta Z_{i}^{B}, \\
\delta Z_{i}^{Z} & =c_{W}^{2} \delta Z_{i}^{W}+s_{W}^{2} \delta Z_{i}^{B}, \\
\delta Z_{i}^{\gamma Z} & =\frac{c_{W} s_{W}}{c_{W}^{2}-s_{W}^{2}}\left(\delta Z_{i}^{Z}-\delta Z_{i}^{\gamma}\right), \\
\delta Z_{V}^{f} & =\left(\delta Z_{L}^{f}+\delta Z_{R}^{f}\right) / 2, \quad \delta Z_{A}^{f}=\left(\delta Z_{L}^{f}-\delta Z_{R}^{f}\right) / 2 .
\end{aligned}
$$


The renormalized self-energies are obtained from unrenormalized ones by adding appropriate counterterms:

$$
\begin{aligned}
\hat{\Sigma}^{\gamma}\left(p^{2}\right) & =\Sigma^{\gamma}\left(p^{2}\right)+\delta Z_{2}^{\gamma} p^{2}, \\
\hat{\Sigma}^{Z}\left(p^{2}\right) & =\Sigma^{Z}\left(p^{2}\right)-\delta M_{Z}^{2}+\delta Z_{2}^{Z}\left(p^{2}-M_{Z}^{2}\right), \\
\hat{\Sigma}^{W}\left(p^{2}\right) & =\Sigma^{W}\left(p^{2}\right)-\delta M_{W}^{2}+\delta Z_{2}^{W}\left(p^{2}-M_{W}^{2}\right), \\
\hat{\Sigma}^{\gamma Z}\left(p^{2}\right) & =\Sigma^{\gamma Z}\left(p^{2}\right)-\delta Z_{2}^{\gamma Z} p^{2}+\left(\delta Z_{1}^{\gamma Z}-\delta Z_{2}^{\gamma Z}\right) M_{Z}^{2}, \\
\hat{\Sigma}^{f}(p) & =\not p\left(\Sigma_{V}^{f}\left(p^{2}\right)+\delta Z_{V}^{f}\right)+\not p \gamma_{5}\left(\Sigma_{A}^{f}\left(p^{2}\right)-\delta Z_{A}^{f}\right) \\
& +m_{f}\left(\Sigma_{S}^{f}\left(p^{2}\right)-\delta Z_{V}^{f}-\frac{\delta m_{f}}{m_{f}}\right),
\end{aligned}
$$

where $\Sigma_{V}^{f}, \Sigma_{A}^{f}$ and $\Sigma_{S}^{f}$ are vector, axial vector and scalar part of the fermion selfenergy, respectively (see Eq. 6.41).

The renormalized electromagnetic, weak neutral and charged current vertices are given by

$$
\begin{aligned}
\hat{\Gamma}^{\gamma f f}= & \Gamma^{\gamma f f}+i e_{137} \gamma_{\mu}\left(\delta Z_{1}^{\gamma}-\delta Z_{2}^{\gamma}+\delta Z_{V}^{f}-\delta Z_{A}^{f} \gamma_{5}\right) \\
- & i e_{137} \gamma_{\mu}\left(v_{f}-a_{f} \gamma_{5}\right)\left(\delta Z_{1}^{\gamma Z}-\delta Z_{2}^{\gamma Z}\right) \\
\hat{\Gamma}^{Z f f}= & \Gamma^{Z f f}+i e_{137} \gamma_{\mu}\left(v_{f}-a_{f} \gamma_{5}\right)\left(\delta Z_{1}^{Z}-\delta Z_{2}^{Z}\right)-i e_{137} \gamma_{\mu}\left(\delta Z_{1}^{\gamma Z}-\delta Z_{2}^{\gamma Z}\right) \\
& +i e_{137} \gamma_{\mu}\left(v_{f} \delta Z_{V}^{f}+a_{f} \delta Z_{A}^{f}\right)-i e_{137} \gamma_{\mu} \gamma_{5}\left(v_{f} \delta Z_{A}^{f}+a_{f} \delta Z_{V}^{f}\right), \\
\hat{\Gamma}^{W l \nu}= & \Gamma^{W l \nu}+i \frac{e_{137}}{2 \sqrt{2} s_{W}} \gamma_{\mu}\left(1-\gamma_{5}\right)\left(1+\delta Z_{1}^{W}-\delta Z_{2}^{W}+\delta Z_{L}^{f}\right) .
\end{aligned}
$$

The renormalization constants are obtained from the OS renormalization conditions Eqs. 4.48 4.49 (we show only constants needed for our calculations):

$$
\begin{aligned}
\delta M_{W}^{2} & =\operatorname{Re} \Sigma^{W}\left(M_{W}^{2}\right) \\
\delta M_{Z}^{2} & =\operatorname{Re} \Sigma^{Z}\left(M_{Z}^{2}\right)
\end{aligned}
$$




$$
\begin{aligned}
\delta Z_{2}^{\gamma} & =-\frac{\partial \Sigma^{\gamma}}{\partial p^{2}}(0), \\
\delta Z_{1}^{\gamma} & =-\frac{\partial \Sigma^{\gamma}}{\partial p^{2}}(0)-\frac{s_{W}}{c_{W}} \frac{\Sigma^{\gamma Z}(0)}{M_{Z}^{2}}, \\
\delta Z_{2}^{Z} & =-\frac{\partial \Sigma^{\gamma}}{\partial p^{2}}(0)-2 \frac{c_{W}^{2}-s_{W}^{2}}{s_{W} c_{W}} \frac{\Sigma^{\gamma Z}(0)}{M_{Z}^{2}}+\frac{c_{W}^{2}-s_{W}^{2}}{s_{W}^{2}}\left(\frac{\delta M_{Z}^{2}}{M_{Z}^{2}}-\frac{\delta M_{W}^{2}}{M_{W}^{2}}\right), \\
\delta Z_{1}^{Z} & =-\frac{\partial \Sigma^{\gamma}}{\partial p^{2}}(0)-\frac{3 c_{W}^{2}-2 s_{W}^{2}}{s_{W} c_{W}} \frac{\Sigma^{\gamma}(0)}{M_{Z}^{2}}+\frac{c_{W}^{2}-s_{W}^{2}}{s_{W}^{2}}\left(\frac{\delta M_{Z}^{2}}{M_{Z}^{2}}-\frac{\delta M_{W}^{2}}{M_{W}^{2}}\right), \\
\delta Z_{2}^{W} & =-\frac{\partial \Sigma^{\gamma}}{\partial p^{2}}(0)-2 \frac{c_{W}}{s_{W}} \frac{\Sigma^{\gamma Z}(0)}{M_{Z}^{2}}+\frac{c_{W}^{2}}{s_{W}^{2}}\left(\frac{\delta M_{Z}^{2}}{M_{Z}^{2}}-\frac{\delta M_{W}^{2}}{M_{W}^{2}}\right), \\
\delta Z_{V}^{f} & =-\Sigma_{V}^{f}\left(m_{f}^{2}\right)-m_{f}^{2}\left[2 \Sigma_{V}^{f^{\prime}}\left(m_{f}^{2}\right)+2 \Sigma_{S}^{f^{\prime}}\left(m_{f}^{2}\right)\right], \quad \Sigma_{V, S}^{f^{\prime}}\left(m_{f}^{2}\right)=\frac{\partial \Sigma_{V, S}^{f}}{\partial p^{2}}\left(m_{f}^{2}\right), \\
\delta Z_{A}^{f} & =+\Sigma_{A}^{f}\left(m_{f}^{2}\right) .
\end{aligned}
$$

\section{E.2 Unrenormalized self-energies in the SM}

Below we present complete SM gauge boson self-energies corresponding to Figs. 6.2 - 6.5. They were calculated in Ref. [34]. For the definition of the function $F$ see Sec. E.3; for the definition of the $\Delta$ factors see Eq. D.9; $s=p^{2}$, where $p$ is the 4-momentum of the gauge boson; $w=M_{W}^{2}, z=M_{Z}^{2}, h=M_{H}^{2}$.

$$
\begin{aligned}
\Sigma^{\gamma}(s) & =\frac{\alpha}{4 \pi}\left\{\frac{4}{3} \sum_{f} Q_{f}^{2}\left[s \Delta_{f}+\left(s+2 m_{f}^{2}\right) F\left(p ; m_{f}, m_{f}\right)-\frac{s}{3}\right]\right. \\
& \left.-3 s \Delta_{W}-(3 s+4 w) F\left(p ; M_{W}, M_{W}\right)\right\} \\
\Sigma^{\gamma Z}(s) & =\frac{\alpha}{4 \pi}\left\{-\frac{4}{3} \sum_{f} Q_{f} v_{f}\left[s \Delta_{f}+\left(s+2 m_{f}^{2}\right) F\left(p ; m_{f}, m_{f}\right)-\frac{s}{3}\right]\right. \\
& +\frac{1}{c_{W} s_{W}}\left[\left(3 c_{W}^{2}+\frac{1}{6}\right) s+2 w\right] \Delta_{W} \\
& \left.+\frac{1}{c_{W} s_{W}}\left[\left(3 c_{W}^{2}+\frac{1}{6}\right) s+\left(4 c_{W}^{2}+\frac{4}{3}\right) w\right] F\left(p ; M_{W}, M_{W}\right)+\frac{s}{9 c_{W} s_{W}}\right\}
\end{aligned}
$$




$$
\begin{aligned}
& \Sigma^{Z}(s)=\frac{\alpha}{4 \pi}\left\{\frac{4}{3} \sum_{l=e, \mu, \tau} 2 a_{l}^{2} s\left(\Delta_{l}+\frac{5}{3}-\ln \left(-\frac{s}{m_{l}^{2}}-i \epsilon\right)\right)\right. \\
& +\frac{4}{3} \sum_{f \neq \nu}\left[\left(v_{f}^{2}+a_{f}^{2}\right)\left(s \Delta_{f}+\left(s+2 m_{f}^{2}\right) F\left(p ; m_{f}, m_{f}\right)-\frac{s}{3}\right)\right. \\
& \left.-\frac{3}{8 c_{W}^{2} s_{W}^{2}} m_{f}^{2}\left(\Delta_{f}+F\left(p ; m_{f}, m_{f}\right)\right)\right] \\
& +\left[\left(3-\frac{19}{6 s_{W}^{2}}+\frac{1}{6 c_{W}^{2}}\right) s+\left(4+\frac{1}{c_{W}^{2}}-\frac{1}{s_{W}^{2}}\right) M_{Z}^{2}\right] \Delta_{W} \\
& +\left[\left(-c_{W}^{4}(40 s+80 w)+\left(c_{W}^{2}-s_{W}^{2}\right)^{2}(8 w+s)+12 w\right) F\left(p ; M_{W}, M_{W}\right)\right. \\
& +\left(10 z-2 h+s+\frac{(h-z)^{2}}{s}\right) F\left(p ; M_{H}, M_{Z}\right)-2 h \ln \frac{h}{w}-2 z \ln z w \\
& +(10 z-2 h+s)\left(1-\frac{h+z}{h-z} \ln \frac{M_{H}}{M_{Z}}-\ln \frac{M_{H} M_{Z}}{w}\right) \\
& \left.\left.+\frac{2}{3} s\left(1+\left(c_{W}^{2}-s_{W}^{2}\right)^{2}-4 c_{W}^{2}\right)\right] \frac{1}{12 c_{W}^{2} s_{W}^{2}}\right\}, \\
& \Sigma^{W}(s)=\frac{\alpha}{4 \pi} \frac{1}{s_{W}^{2}}\left\{\frac { 1 } { 3 } \sum _ { l = e , \mu , \tau } \left[\left(s-\frac{3}{2} m_{l}^{2}\right) \Delta_{l}\right.\right. \\
& \left.+\left(s-\frac{m_{l}^{2}}{2}-\frac{m_{l}^{4}}{2 s}\right) F\left(p ; 0, m_{l}\right)+\frac{2}{3} s-\frac{m_{l}^{2}}{2}\right] \\
& +\sum_{q-\text { doublets }} \frac{1}{3}\left[\frac{\Delta_{+}}{2}\left(s-\frac{5}{2} m_{+}^{2}+\frac{m_{-}^{2}}{2}\right)+\frac{\Delta_{-}}{2}\left(s-\frac{5}{2} m_{-}^{2}+\frac{m_{+}^{2}}{2}\right)\right. \\
& +\left(s-\frac{m_{+}^{2}+m_{-}^{2}}{2}-\frac{\left(m_{+}^{2}-m_{-}^{2}\right)^{2}}{2 s}\right) F\left(p ; m_{+}, m_{-}\right) \\
& \left.+\left(s-\frac{m_{+}^{2}+m_{-}^{2}}{2}\right)\left(1-\frac{m_{+}^{2}+m_{-}^{2}}{m_{+}^{2}-m_{-}^{2}} \ln m_{+} m_{-}\right)-\frac{s}{3}\right] \\
& -\left[\frac{19}{2} s+3 w\left(1-\frac{s_{W}^{2}}{c_{W}^{2}}\right)\right] \frac{\Delta_{W}}{3} \\
& +\left[s_{W}^{4} z-\frac{c_{W}^{2}}{3}\left(7 z+7 w+10 s-2 \frac{(z-w)^{2}}{s}\right)\right. \\
& \left.-\frac{1}{6}\left(w+z-\frac{s}{2}-\frac{(z-w)^{2}}{2 s}\right)\right] F\left(p ; M_{Z}, M_{W}\right) \\
& +\frac{s_{W}^{2}}{3}\left(-4 w-10 s+\frac{2 w^{2}}{s}\right) F\left(p ; 0, M_{W}\right) \\
& +\frac{1}{6}\left(5 w-h+\frac{s}{2}+\frac{(h-w)^{2}}{2 s}\right) F\left(p ; M_{H}, M_{W}\right)
\end{aligned}
$$




$$
\begin{aligned}
& +\left[\frac{c_{W}^{2}}{3}(7 z+7 w+10 s-4(z-w))-s_{W}^{4} z+\frac{1}{6}\left(2 w-\frac{s}{2}\right)\right] \frac{3 z}{z-w} \ln \frac{z}{w} \\
& -\left(\frac{2}{3} w+\frac{s}{12}\right) \frac{h}{h-w} \ln \frac{h}{w}-\frac{c_{W}^{2}}{3}\left(7 z+7 w+\frac{32}{3} s\right)+s_{W}^{4} z \\
& \left.+\frac{1}{6}\left(\frac{5}{3} s+4 w-z-h\right)-\frac{s_{W}^{2}}{3}\left(4 w+\frac{32}{3} s\right)\right\} .
\end{aligned}
$$

\section{E.3 't Hooft scalar integrals}

Here we define various $C, B, A$ and $F$ functions and reduce them to scalar integrals $C_{0}, B_{0}$ and $A_{0}$. For the calculation of $C_{0}$ and $B_{0}$ we refer the reader to the original work of 't Hooft and Veltman, Ref. [51].

The $C_{0}$ function is defined as (with finite parts indicated by the superscript):

$$
\begin{aligned}
C_{0}\left(m_{1}, m_{2}, m_{3}\right) & \equiv C_{0}\left(p_{1}, p_{2} ; m_{1}, m_{2}, m_{3}\right) \equiv C_{0}^{f i n}\left(m_{1}, m_{2}, m_{3}\right) \\
& =-\int \frac{d^{n} q}{i \pi^{2}} \frac{1}{D},
\end{aligned}
$$

where

$$
D=\left(q^{2}-m_{1}^{2}+i \epsilon\right)\left[\left(q-p_{1}\right)^{2}-m_{2}^{2}+i \epsilon\right]\left[\left(q-p_{1}-p_{2}\right)^{2}-m_{3}^{2}+i \epsilon\right]
$$

The functions $C_{i j}$ are defined by:

$$
\begin{aligned}
C_{\mu} & =-\int \frac{d^{n} q}{i \pi^{2}} \frac{q_{\mu}}{D}=-p_{1 \mu} C_{11}-p_{2 \mu} C_{12}, \\
C_{\mu \nu} & =-\int \frac{d^{n} q}{i \pi^{2}} \frac{q_{\mu}}{D} \\
& =p_{1 \mu} p_{1 \nu} C_{21}+p_{2 \mu} p_{2 \nu} C_{22}+\left(p_{1 \mu} p_{2 \nu}+p_{1 \nu} p_{2 \mu}\right) C_{23}-g_{\mu \nu} C_{24} .
\end{aligned}
$$

The functions $C_{11}, C_{24}, C_{23}$ are reduced (in the limit $p_{1}^{2}=p_{2}^{2}=m_{l}^{2} \ll\left(p_{1}+p_{2}\right)^{2}=$ 
$M_{Z}^{2}$, applicable for our considerations of the leptonic decays of the Z boson) to:

$$
\begin{aligned}
C_{11}\left(m_{1}, m_{2}, m_{3}\right) & =C_{11}^{f i n}\left(m_{1}, m_{2}, m_{3}\right)=-\frac{1}{M_{Z}^{2}}\left[f_{2} C_{0}\left(m_{1}, m_{2}, m_{3}\right)\right. \\
& \left.-B_{0}^{f i n}\left(p_{1}+p_{2} ; m_{1}, m_{3}\right)+B_{0}^{f i n}\left(p_{1} ; m_{1}, m_{2}\right)\right] \\
C_{24}\left(m_{1}, m_{2}, m_{3}\right) & =\frac{1}{4} \Delta+C_{24}^{f i n}\left(m_{1}, m_{2}, m_{3}\right), \\
C_{24}^{f i n}\left(m_{1}, m_{2}, m_{3}\right) & =\left[m_{1}^{2} C_{0}\left(m_{1}, m_{2}, m_{3}\right)+f_{1} C_{11}\left(m_{1}, m_{2}, m_{3}\right)\right. \\
& \left.+B_{1}^{f i n}\left(p_{1}+p_{2} ; m_{1}, m_{3}\right)\right]\left(-\frac{1}{2}\right)+\frac{1}{4}, \\
& =C_{23}^{f i n}\left(m_{1}, m_{2}, m_{3}\right)=-\frac{1}{M_{Z}^{2}}\left[B_{1}^{f i n}\left(p_{1}+p_{2} ; m_{1}, m_{3}\right)\right. \\
& \left.+B_{0}^{f i n}\left(p_{2} ; m_{2}, m_{3}\right)+f_{1} C_{11}\left(m_{1}, m_{2}, m_{3}\right)\right] \\
& +C_{24}^{f i n}\left(m_{1}, m_{2}, m_{3}\right) \frac{2}{M_{Z}^{2}},
\end{aligned}
$$

where

$$
\begin{aligned}
& f_{2}=M_{Z}^{2}+m_{2}^{2}-m_{3}^{2} \\
& f_{1}=m_{1}^{2}-m_{2}^{2}
\end{aligned}
$$

The functions $B_{0}, B_{1}$ are defined as:

$$
\begin{aligned}
B_{0}\left(p ; m_{1}, m_{2}\right) & =\int \frac{d^{n} q}{i \pi^{2}} \frac{1}{\left(q^{2}-m_{1}^{2}+i \epsilon\right)\left[(q-p)^{2}-m_{2}^{2}+i \epsilon\right]} \\
& =\Delta+B_{0}^{f i n}\left(p ; m_{1}, m_{2}\right), \\
B_{0}^{f i n}\left(p ; m_{1}, m_{2}\right) & =-\int_{0}^{1} d x \ln \left[p^{2} x^{2}+m_{1}^{2}-\left(p^{2}+m_{1}^{2}-m_{2}^{2}\right) x\right], \\
B_{\mu}\left(p ; m_{1}, m_{2}\right) & =\int \frac{d^{n} q}{i \pi^{2}} \frac{q_{\mu}}{\left(q^{2}-m_{1}^{2}+i \epsilon\right)\left[(q-p)^{2}-m_{2}^{2}+i \epsilon\right]}=-p_{\mu} B_{1},
\end{aligned}
$$




$$
\begin{aligned}
B_{1}\left(p ; m_{1}, m_{2}\right) & =-\frac{1}{2} \Delta+B_{1}^{f i n}\left(p ; m_{1}, m_{2}\right) \\
B_{1}^{f i n}\left(p ; m_{1}, m_{2}\right) & =\int_{0}^{1} d x \ln \left[p^{2} x^{2}+m_{1}^{2}-\left(p^{2}+m_{1}^{2}-m_{2}^{2}\right) x\right] x \\
B_{\mu \nu}\left(p ; m_{1}, m_{2}\right) & =\int \frac{d^{n} q}{i \pi^{2}} \frac{q_{\mu} q_{\nu}}{\left(q^{2}-m_{1}^{2}+i \epsilon\right)\left[(q-p)^{2}-m_{2}^{2}+i \epsilon\right]} \\
& =p_{\mu} p_{\nu} B_{21}-g_{\mu \nu} B_{22} .
\end{aligned}
$$

The functions $B_{1}, B_{21}$ and $B_{22}$ can be reduced to

$$
\begin{aligned}
B_{1} & =\frac{1}{2 p^{2}}\left\{-A_{0}\left(m_{1}\right)+A_{0}\left(m_{2}\right)-\left(p^{2}+m_{1}^{2}-m_{2}^{2}\right) B_{0}\right\}, \\
B_{21} & =\frac{1}{3 p^{2}}\left\{-A_{0}\left(m_{2}\right)-2\left(p^{2}+m_{1}^{2}-m_{2}^{2}\right) B_{1}-m_{1}^{2} B_{0}-1 / 2\left(m_{1}^{2}+m_{2}^{2}-p^{2} / 3\right)\right\}, \\
B_{22} & =\frac{1}{6}\left\{+A_{0}\left(m_{2}\right)-\left(p^{2}+m_{1}^{2}-m_{2}^{2}\right) B_{1}-2 m_{1}^{2} B_{0}-\left(m_{1}^{2}+m_{2}^{2}-p^{2} / 3\right)\right\} .(\mathrm{E} .15)
\end{aligned}
$$

The functions $A$ are defined as

$$
\begin{aligned}
A_{0}(m) & =-\int \frac{d^{n} q}{i \pi^{2}} \frac{1}{(q-p)^{2}-m^{2}}=-\int \frac{d^{n} q}{i \pi^{2}} \frac{1}{q^{2}-m^{2}} \\
& =-m^{2}\left(\Delta-\ln m^{2}+1\right), \\
A_{\mu}(p, m) & =\int \frac{d^{n} q}{i \pi^{2}} \frac{q_{\mu}}{(q-p)^{2}-m^{2}}=-p_{\mu} A_{0}(m) .
\end{aligned}
$$

Relations between $\mathrm{F}$ and $\mathrm{B}$ functions:

$$
\begin{aligned}
F\left(p ; m_{1}, m_{2}\right) & =-1+\frac{m_{1}^{2}+m_{2}^{2}}{m_{1}^{2}-m_{2}^{2}} \ln \frac{m_{1}}{m_{2}}+\ln m_{1}+\ln m_{2}+B_{0}\left(p ; m_{1}, m_{2}\right), \\
F(p ; 0, m) & =-1+\ln m^{2}+B_{0}(p ; 0, m) \\
B_{1}\left(p ; m_{1}, m_{2}\right) & =\frac{m_{2}^{2}-m_{1}^{2}}{2} \frac{F\left(p ; m_{1}, m_{2}\right)}{p^{2}}-\frac{1}{2} B_{0}\left(p ; m_{1}, m_{2}\right) .
\end{aligned}
$$


For $s=p^{2}$ small with respect to $m_{1}^{2}, m_{2}^{2}, m^{2}$, we have

$$
\begin{aligned}
F\left(p ; m_{1}, m_{2}\right) & =\frac{s}{\left(m_{1}^{2}-m_{2}^{2}\right)^{2}}\left[\frac{m_{1}^{2}+m_{2}^{2}}{2}-\frac{m_{1}^{2} m_{2}^{2}}{m_{1}^{2}-m_{2}^{2}} \ln \frac{m_{1}^{2}}{m_{2}^{2}}\right], \\
B_{0}\left(p ; m_{1}, m_{2}\right) & =1-\frac{m_{1}^{2}+m_{2}^{2}}{m_{1}^{2}-m_{2}^{2}} \ln \frac{m_{1}}{m_{2}}-\ln m_{1}-\ln m_{2}+O(s), \\
B_{0}(p ; 0, m) & =1-2 \ln m+O(s), \\
B_{1}\left(p ; m_{1}, m_{2}\right) & =\frac{1}{2} \frac{1}{m_{2}^{2}-m_{1}^{2}}\left[\frac{m_{1}^{2}+m_{2}^{2}}{2}-\frac{m_{1}^{2} m_{2}^{2}}{m_{1}^{2}-m_{2}^{2}} \ln \frac{m_{1}^{2}}{m_{2}^{2}}\right]-\frac{1}{2} B_{0}\left(p ; m_{1}, m_{2}\right), \\
B_{1}(p ; 0, m) & =-\frac{1}{4}+\ln m+O(s), \\
B_{1}(p ; m, 0) & =-\frac{3}{4}+\ln m+O(s) .
\end{aligned}
$$

Finally, in photon loops we encounter functions with regularized photon mass $m_{\lambda} \rightarrow 0:$

$$
\begin{aligned}
\left.B_{0}\left(p ; m_{\lambda}, m_{l}\right)\right|_{p^{2}=m_{l}^{2}} & =2-2 \ln m_{l}, \\
\left.B_{1}\left(p ; m_{\lambda}, m_{l}\right)\right|_{p^{2}=m_{l}^{2}} & =-\frac{1}{2}+\ln m_{l}, \\
\left.B_{1}\left(p ; m_{l}, m_{\lambda}\right)\right|_{p^{2}=m_{l}^{2}} & =-\frac{3}{2}+\ln m_{l}, \\
\left.\frac{\partial B_{0}}{\partial p^{2}}\left(p ; m_{\lambda}, m_{l}\right)\right|_{p^{2}=m_{l}^{2}} & =\frac{1}{m_{l}^{2}}\left(-1-\ln \frac{m_{\lambda}}{m_{l}}\right), \\
\left.\frac{\partial B_{1}}{\partial p^{2}}\left(p ; m_{\lambda}, m_{l}\right)\right|_{p^{2}=m_{l}^{2}} & =-\frac{1}{2 m_{l}^{2}} .
\end{aligned}
$$




\section{Bibliography}

[1] David Griffiths, Introduction to Elementary Particles (John Wiley, 1987).

[2] Particle Data Group, Phys. Rev. D50, 1173 (1994).

[3] S.L. Glashow, Nucl. Phys. 22, 579 (1961).

[4] S. Weinberg, Phys. Rev. Lett. 19, 1264 (1967).

[5] A. Salam, in Elementary Particle Theory, ed. N. Svartholm (Almquist and Wiksells, Stockholm, 1969), p. 367.

[6] S.L. Glashow, J. Iliopoulos and L. Maiani, Phys. Rev. D2, 1285 (1970).

[7] A classic paper on GUT's is by Georgi and Glashow: H. Georgi and S. L. Glashow, Phys. Rev. Lett. 32, 438 (1974); the following two books review the further development in the field: R. N. Mohapatra, Unification and Supersymmetry (Springer-Verlag, 1986); G. G. Ross, Grandunified Theories (BenjaminCummings, 1985).

[8] M. Gell-Mann, P. Ramond, and R. Slansky, in Supergravity, eds. P. Van Nieuwenhuizen and D. Freedman (North Holland, 1979), p. 315; T. Yanagida, in Proc. of the Workshop on Unified Theory and Baryon Number of the Universe, eds. O. Swada and A. Sugamoto (KEK, 1979), p. 95; R.N. Mohapatra and G. Senjanovic, Phys. Rev. Lett. 44, 912 (1980). 
[9] The original see-saw mechanism of Gell-Mann et al. and Yanagida was formulated in the context of $S U(2)_{L} \times S U(2)_{R} \times U(1)$ group. E. Witten showed the see-saw mechanism works also in the minimal SO(10) GUT: E. Witten, Phys. Lett. B91, 81 (1980).

[10] Y. Chikashige, R.N. Mohapatra and R.D. Peccei, Phys. Lett. B98, 265 (1981).

[11] Review of the experimental situation may be found in: M. Moe, Int. J. Mod. Phys. E2, 507 (1993).

[12] R. Davis Jr., in Frontiers of Neutrino Astrophysics, ed. by Y. Suzuki and K. Nakamura (Universal Academy Press, Inc., Tokyo, Japan, 1993), p. 47; K. S. Hirata, et al., Phys. Rev. D44, 2241 (1991); P. Anselmann, et al., Phys. Lett. B285, 376 (1992); P. Anselmann, et al., Phys. Lett. B314, 445 (1993); A. I. Abazov, et al., Phys. Rev. Lett. 67, 3332 (1991b); J. N. Abdurashitov, et al., Phys. Lett. B328, 234 (1994).

[13] S.P. Mikheyev and A. Y. Smirnov, Nuovo Cim. 9C, 17 (1986).

[14] E. L. Wright, et al., Astrophys. J. 396, L13 (1992); R. Schaefer and Q. Shafi, BA-92-28 (1992); J. A. Holtzman and J. Primack, Astrophys. J. 405, 428 (1993).

[15] R.N. Mohapatra and P.B. Pal, Massive Neutrinos in Particle Physics and Astrophysics (World Scientific, 1991).

[16] R.N. Mohapatra, Invited talk at Int. Conf. on Neutrino Telescopes, Venice, Italy, Feb 1994; e-Print Archive: hep-ph/9405358.

[17] R.N. Mohapatra and J. W. F. Valle, Phys. Rev. D34, 1642 (1986).

[18] M. C. Gonzalez-Garcia and J. W. F. Valle, Phys. Lett. B216, 360 (1989).

[19] A. Pilaftsis, Z. Phys. C55, 275 (1992). 
[20] L. N. Chang, D. Ng and J. N. Ng, Phys. Rev. D50, 4589 (1994).

[21] V. Barger and R. Phillips, Collider Physics (Addison-Wesley, 1987).

[22] N. Cabibbo, Phys. Rev. Lett. 10, 531 (1963); M. Kobayashi and T. Maskawa, Progr. Theor. Phys. 42, 652 (1973).

[23] For a review, see J. L. Hewett and T. G. Rizzo, Phys. Rep. 183, 193 (1989).

[24] Ch. Berger, et al. (FREJUS Collab.), Z. Phys. C50, 385 (1991); R. BeckerSzendy, et al. (IMB-3 Collab.), Phys. Rev. D42, 2974 (1990); K. Hirata, et al. (Kamiokande Collab.), Phys. Lett. B220, 308 (1989).

[25] M. Green and J. Schwarz, Phys. Lett. 149B, 117 (1984); 151B, 21 (1985).

[26] J. Bernabéu, A. Santamaria, J. Vidal, A. Mendez, and J. W. F. Valle, Phys. Lett. B187, 303 (1987).

[27] D. Wyler and L. Wolfenstein, Nucl. Phys. B218, 205 (1983).

[28] B. Kayser, in The Physics of Massive Neutrinos (World Scientific, 1989), p. 87.

[29] G.C. Branco, M.N. Rebelo and J. W. F. Valle, Phys. Lett. B225, 385 (1989).

[30] M. Dittmar, A. Santamaria, M. C. Gonzalez-Garcia and J. W. F. Valle, Nucl. Phys. B332, 1 (1989); M. C. Gonzalez-Garcia, A. Santamaria and J. W. F. Valle, Nucl. Phys. B342, 108 (1990).

[31] M. Gronau, C. N. Leung and J. L. Rosner, Phys. Rev. D29, 2539 (1984).

[32] G. Bhattacharya, in Beyond the Standard Model III, Eds. S. Godfrey and P. Kalyniak (World Scientific, 1993), p. 463.

[33] E. Nardi, E. Roulet and D. Tommasini, Phys. Lett. B327, 319 (1994). 
[34] W. Hollik, Fortschr. Phys. 38, 165 (1990).

[35] W. Hollik, H. Bohm and H. Spiesberger, Fortschr. Phys. 34, 687 (1986).

[36] K. Aoki, Z. Hioki, R. Kawabe, M. Konuma and T. Muta, Suppl. Progr. Theor. Phys. No. 73, 1 (1982).

[37] F. Jegerlehner, in Testing the Standard Model, Proceedings of the 1990 TASI in Elementary Particle Physics, ed. M. Cvetic and P. Langacker (World Scientific, 1991), p. 476.

[38] L.D. Faddeev and V.N. Popov, Phys. Lett. 25B, 29 (1967).

[39] G. 't Hooft, Nucl. Phys. B35, 167 (1971).

[40] G. 't Hooft and M. Veltman, Nucl. Phys. B44, 189 (1972).

[41] J. C. Ward, Phys. Rev. 78, 1824 (1950); J. C. Taylor, Nucl. Phys. B33, 436 (1971); A. A. Slavnov, Theor. Math. Phys. 10, 99 (1972).

[42] D. Ng and J. N. Ng, Phys. Lett. B331, 371 (1994).

[43] M. C. Gonzalez-Garcia and J. W. F. Valle, Mod. Phys. Lett. A 7, 477 (1991).

[44] A. Ilakovac and A. Pilaftsis, Nucl.Phys. B437, 491 (1995).

[45] D. Tommasini, G. Barenboim, J. Bernabeu and C. Jarlskog, Nucl.Phys. B444, $451(1995)$.

[46] N. Rius, J.W.F. Valle, Phys. Lett. B246, 249 (1990).

[47] J.G. Korner, A. Pilaftsis and K. Schilcher, Phys. Lett. B300, 381 (1993).

[48] A. Pilaftsis, Mod. Phys. Lett. A9, 3595 (1994). 
[49] J. Bernabéu, J. G. Körner, A. Pilaftsis, and K. Schilcher Phys. Rev. Lett. 71, 2695 (1993).

[50] G. Bhattacharya, P. Kalyniak and I. Melo, Phys. Rev. D51, 3569 (1995).

[51] G. 't Hooft and M. Veltman, Nucl. Phys. B153, 365 (1979).

[52] A. Olchevski, plenary talk at the International Europhysics Conference on High Energy Physics, Brussels, 27 July - 2 August, 1995.

[53] F. Abe, et al. (the CDF collaboration), Phys.Rev.Lett. 75, 11 (1995).

[54] T.P. Cheng and Ling-Fong Li, Phys. Rev. D44, 1502 (1991).

[55] John Jowett, in Proc. of the Tau-Charm Factory Workshop (1989), p. 7., SLAC Report 343 (1989); Y. S. Tsai, Phys.Rev. D51, 3172 (1995).

[56] D. Albert, W. J. Marciano and D. Wyler, Nucl. Phys. B166, 460 (1980).

[57] FORTRAN version of the Ref. [34], used by CERN experimentalists for the simulations of LEP data.

[58] Ref. [37, p. 526.

[59] T. Appelquist and J. Carrazone, Phys. Rev. D11, 2856 (1975).

[60] J. F. Donoghue, E. Golowich and B. R. Holstein, Dynamics of the Standard Model (Cambridge University Press, 1992).

[61] F. Abe, et al. (the CDF collaboration), Phys. Rev. Lett. 74, 2626 (1995).

[62] V. A. Novikov, L. B. Okun and M. I. Vysotsky, Mod. Phys. Lett. A8, 2529 (1993).

[63] R. K. Carnegie, private communication. 
[64] B. A. Kniehl and A. Pilaftsis, e-Print Archive: hep-ph/9601390. 